\title{
Aerothermodynamische Untersuchung einer Wiedereintrittskonfiguration und ihrer Komponenten in einem impulsbetriebenen Hochenthalpie-Stoßkanal
}

\author{
Dissertation \\ zur Erlangung des Doktorgrades \\ der Mathematisch-Naturwissenschaftlichen Fakultäten \\ der Georg-August-Universität zu Göttingen
}

vorgelegt von

Jan Martinez Schramm

aus Göttingen

Göttingen 2008 


\section{Übersicht}

Beim Wiedereintritt in die Erdatmosphäre wird in einer Flughöhe von $60 \mathrm{~km}$ bis $80 \mathrm{~km}$ über Grund die hohe kinetische Energie eines Raumfahrzeuges überwiegend in thermische Energie des vor dem Körper befindlichen Gases umgewandelt und führt so zur aerodynamischen Erwärmung des Raumfahrzeuges. Diese Erwärmung erreicht solche Größenordnungen, dass sie für die Auslegung und den sicheren Betrieb eines Raumfahrzeuges eine maßgebliche Bedeutung hat. Durch die hohe thermische Energie des Gases vor dem Körper tritt neben anderen chemischen Reaktionen vor allem Dissoziation der Gasmoleküle auf. Im interessierenden Flugbereich ist die Zeitdauer des Ablaufes der chemischen Reaktionen von der Größenordnung der Verweildauer des Fluides in einem lokalen thermodynamischen Zustand. Deshalb sind Relaxationseffekte von Bedeutung.

Hochenthalpie-Windkanäle können die Umströmung eines Raumfahrzeuges in diesem Flugbereich im Experiment kurzzeitig (Messzeit ca. 1 ms) nachbilden. Zur Übertragung der realen Flugsituation in das Windkanalexperiment am skalierten Modell dient die strömungsmechanische Ähnlichkeitstheorie. Eine wichtige Kennzahl, neben der Machzahl und der Reynoldszahl, ist der binäre Skalierungsparameter, das Produkt aus Gasdichte und einer Bezugslänge, der die Dissoziationsrate bestimmt.

Zur Auslegung eines Raumfahrzeuges werden numerische Verfahren angewandt, um die Umströmung während des Wiedereintrittes zu bestimmen. Zur Validierung der chemischen Modellierung innerhalb dieser Verfahren werden experimentelle Daten aus Versuchen in Hochenthalpie-Windkanälen verwendet.

In dieser Arbeit werden im Hochenthalpiekanal Göttingen (HEG) experimentell Druck und Wärmestromdichte an der Oberfläche eines skalierten Windkanalmodells des Experimentalfahrzeuges X-38 ermittelt und mit numerischen Ergebnissen verglichen und diskutiert. Das X-38 wurde als Technologiedemonstrator für ein zukünftiges Rettungsfahrzeug für die Internationale Raumstation von der NASA in enger Kooperation mit der ESA entworfen und gebaut. Wichtige Strömungsbereiche befinden sich im Bereich der Nase und der Steuerklappen dieser Wiedereintrittskonfiguration. Zur detaillierten Untersuchung der Umströmung dieser Bereiche, die am Modell nur eingeschränkt möglich ist, werden weitere Experimente mit vergrößerten und vereinfachten Windkanalmodellen durchgeführt.

An einem Zylindermodell zur Nachbildung des Nasenbereiches werden Dichtegradienten der Umströmung mit der Methode der holographischen Interferometrie vermessen und mit numerischen Ergebnissen verglichen. Diese Messdaten lassen eine Validierung der Relaxationsmodellierung in den numerischen Verfahren zu.

Die Umströmung der Steuerklappen wird mit einem Doppelrampenmodell nachgebildet. Aufgrund der Größe dieses Modells können Strömungsablösungen zeitlich sichtbar gemacht werden. Ein Vergleich mit gängigen analytischen Modellen für nicht chemisch reagierende Überschallströmungen wird durchgeführt. Für diese Messungen wird eine Hochgeschwindigkeits-Sichtbarmachung aufgebaut und optimiert. 



\section{Inhalt}

Abbildungen vii

Tabellen $\quad$ xi

1 Einleitung $\quad 1$

1.1 Hintergrund und Motivation . . . . . . . . . . . . . . . . . . . . . . . . . . . . . . . .

1.2 Problemstellung und Zielsetzung . . . . . . . . . . . . . . . . . . . . . 5

2 Stand der Forschung $\quad 9$

2.1 Hochtemperatureffekte . . . . . . . . . . . . . . . . . . . . . . . 99 9

2.2 Umströmung eines Zylinders bei hohen Geschwindigkeiten . . . . . . . . . 12

2.3 Wissensstand über die X-38-Konfiguration . . . . . . . . . . . . . . . . . . 13

2.4 Stoß-Grenzschicht-Wechselwirkung . . . . . . . . . . . . . . . . . . . . . . 14

3 Der Hochenthalpiekanal Göttingen 19

3.1 Funktionsweise und Aufbau . . . . . . . . . . . . . . . . . . . . . . . 20

3.2 Durchgeführte Versuche . . . . . . . . . . . . . . . . . . . . . . 26

3.3 Messungen während eines Versuches . . . . . . . . . . . . . . . . . . . . . 27

4 Messtechniken und Modellinstrumentierung 39

4.1 Optische Methoden . . . . . . . . . . . . . . . . . . . . . . . . . . . . 39

4.2 Messung von Oberflächendruck und Wandwärmestromdichte . . . . . . . . . 51

4.3 Verwendete Modelle . . . . . . . . . . . . . . . . . . . . . . . . . 58

5 Diskussion der Messergebnisse $\quad 67$

5.1 Zylindermodell . . . . . . . . . . . . . . . . . . . . . . 67

5.2 X-38-Modell . . . . . . . . . . . . . . . . . . 81

5.3 Doppelrampenmodell . . . . . . . . . . . . . . . . . . . . 89

6 Zusammenfassung und Ausblick 103

$\begin{array}{ll}\text { Literatur } & 105\end{array}$

$\begin{array}{ll}\text { A Thermoelementkalibrierung } & 125\end{array}$

B Fehlerrechnungen 129

B.1 Wärmestromdichtemessung mittels koaxialer Thermoelemente . . . . . . . 129

B.2 Bestimmung der Ablöselänge . . . . . . . . . . . . . . . . . . . . . . . . . . 130

B.3 Bestimmung der Ruheenthalpie $h_{0} \ldots \ldots \ldots \ldots$. . . . . . . . . . . . . 131

B.4 Kontaktkalibrierungsverfahren . . . . . . . . . . . . . . . . . . . 132 



\section{Abbildungen}

1.1 Hochtemperatureffekte beim Wiedereintritt . . . . . . . . . . . . . . . . 2

1.2 Photographische Ansichten des X-38 (V131,NASA Dryden) . . . . . . . . . . 3

1.3 Wiedereintrittstrajektorien in die Erdatmosphäre . . . . . . . . . . . . . . . 4

1.4 Zerlegung der Umströmung eines Raumfahrzeuges in generische Teilbereiche 7

2.1 Temperaturen hinter einem Verdichtungsstoß beim Wiedereintritt . . . . . . 9

2.2 Zylinderumströmung, Topologie und Notation . . . . . . . . . . . . . . . . . 13

2.3 Topologie bei abgelösten Strömungen im Überschall . . . . . . . . . . . . . . . 15

2.4 Umströmung des Rampenmodells . . . . . . . . . . . . . . . . . . . . . . . . 16

3.1 Photographische Ansichten des HEG . . . . . . . . . . . . . . . . . . . . . . . . . 19

3.2 Wellendiagramm des einfaches Stoßrohres . . . . . . . . . . . . . . . . . 20

3.3 Wellendiagramm des HEG . . . . . . . . . . . . . . . . . . . . . . . . . . . 21

3.4 Abhängigkeit der Stoßmachzahl von den Füllbedingungen des Stoßrohres . 22

3.5 Darstellung des HEG . . . . . . . . . . . . . . . . . . . . . . . . . . . 23

3.6 Numerische Simulation des HEG bei Bedingung I . . . . . . . . . . . . . . . . . . 24

3.7 Messung der Kanalbewegung . . . . . . . . . . . . . . . . . . . . . . . . . . . 28

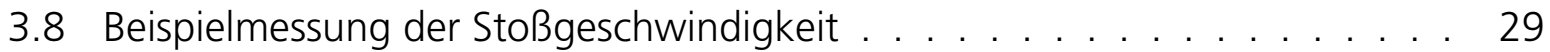

3.9 Aufbau der Hauptmembranstation . . . . . . . . . . . . . . . . . . . . . . . . . 30

3.10 Messung des Berstdruckes . . . . . . . . . . . . . . . . . . . . . . . . . . . 31

3.11 Aufbau der Stoßrohrendwand . . . . . . . . . . . . . . . . . . . . . . . . . . . . . . . . . . . . . . . . . . . . . . . .

3.12 Messung des Reservoirdruckes . . . . . . . . . . . . . . . . . . . . . . . . . . . . . . . . . . . . . . . . . . . . . . 34

3.13 Messungen der permanenten Sonde . . . . . . . . . . . . . . . . . . . . . . . . . . 34

3.14 Normierte Messungen der permanenten Sonde . . . . . . . . . . . . . . . . . 35

3.15 Signalverbesserung der Lanzensonden . . . . . . . . . . . . . . . . . . . 37

3.16 Photos der Lanzensonden . . . . . . . . . . . . . . . . . . . . . . . . . . 37

3.17 Lanzensonde . . . . . . . . . . . . . . . . . . . . . . . . . . . . . . . . 37

3.18 Druckkorrektur für die Lanzensonde . . . . . . . . . . . . . . . . . . . . . . . . . 38

4.1 Schematischer Aufbau der Hochgeschwindigkeits-Sichtbarmachung . . . . . 40

4.2 Auflösung der Hochgeschwindigkeits-Sichtbarmachung . . . . . . . . . . . 41

4.3 Prinzip der interferometrischen Dichtebestimmung . . . . . . . . . . . . . . . 43

4.4 Speicherungsarten für Lichtinformation . . . . . . . . . . . . . . . . . . . . 45

4.5 Schema der holographischen Bildrekonstruktion . . . . . . . . . . . . . . . 46

4.6 Schema des Strahlengangs der holographischen Interferometrie . . . . . . . 47

4.7 Aufnahme und Rekonstruktion bei der holographischen Interferometrie . . . 48

4.8 Beispiel für das Phasenschrittverfahren bei der holographischen Rekonstruktion 50

4.9 Funktionsprinzip der verwendeten Druckaufnehmer . . . . . . . . . . . . . . 52

4.10 Aufbau der verwendeten piezoresistiven Drucksensoren der Firma KULITE . . 53

4.11 Verwendete Drucksensoreinbauten . . . . . . . . . . . . . . . . . . . 53

4.12 Miniaturisiertes Thermoelement der Firma MedTherm . . . . . . . . . . . . 55

4.13 Beispiel für eine Wärmestromdichteauswertung . . . . . . . . . . . . . . . . 57 
4.14 Zylindermodell . . . . . . . . . . . . . . . . . . . . . . . 58

4.15 Sensorik auf dem Zylindermodell . . . . . . . . . . . . . . . . . . . 59

4.16 Oberflächenbearbeitung des Zylindermodells . . . . . . . . . . . . . . 60

4.17 Doppelrampenmodell . . . . . . . . . . . . . . . . . . . . . . . . 61

4.18 Sensorik des Doppelrampenmodells . . . . . . . . . . . . . . . . . . 62

4.19 Modell des X-38 . . . . . . . . . . . . . . . . . . . . . . . 63

4.20 Schnittzeichnung des Windkanalmodells des X-38 . . . . . . . . . . . . 64

4.21 Sensoren auf der Oberfläche des Modells des X-38 . . . . . . . . . . . . . 65

4.22 Explosionszeichnung des Windkanalmodells des X-38 f . . . . . . . 66

5.1 Einfluss der Dreidimensionalität der Strömung auf die Oberflächenmessungen 68

5.2 Ergebnisse der Druckmessung auf dem Zylinder . . . . . . . . . . . . . 70

5.3 Wärmestrommessungen auf dem Zylinder . . . . . . . . . . . . . . . 71

5.4 Zeitliche Sichtbarmachung der Zylinderumströmung bei Bedingung III . . . . 72

5.5 Zeitlicher Verlauf des Zylinder-Stoßabstandes bei Bedingung III und I . . . . . 73

5.6 Einfluss der Dreidimensionalität der Zylinderumströmung . . . . . . . . . . 74

5.7 Phasenverschiebung CFD/Experiment auf der Zylinderstaustromlinie . . . . . 75

5.8 Variation der Stoßabstände mit dem verwendeten Reaktionsratenmodell . . 77

5.9 Abweichungungen der numerischen Stoßabstände vom Experiment . . . . . 77

5.10 Phasenverschiebung CFD/Experiment der Zylinderströmung (Bed. I, Luft/ $/ \mathrm{N}_{2}$ ) . 78

5.11 Phasenverschiebung CFD/Experiment der Zylinderströmung (Bed. III, Luft/ $\mathrm{N}_{2}$ ) 79

5.12 Druckmessung X-38-Modell (Bed. I, $\delta=20^{\circ} / 30^{\circ}$ ) . . . . . . . . . . . . 82

5.13 Druckmessung X-38-Modell (Bed. III, $\left.\delta=20^{\circ} / 30^{\circ}, \beta=5^{\circ}\right) \ldots \ldots . . . . . .83$

5.14 Wärmestromdichtemessung X-38 Modell (Vorkörper und Klappenschacht) . 84

5.15 Wärmestromdichtemessung X-38-Modell $\delta=20^{\circ}$ und $30^{\circ} \ldots \ldots$

5.16 Numerisches Stromlinienbild auf dem X-38-Modell . . . . . . . . . . . . 85

5.17 Wärmestromdichtemessung X-38-Modell (Bed. I,III, $\delta=20^{\circ} / 30^{\circ}, \beta=5^{\circ}$ ) . . . . 86

5.18 Wärmestromdichtemessung $X-38$-Modell (Bed. I,III, Spannw.) . . . . . . . . 87

5.19 Wärmestromdichtemessung X-38-Modell (Bed. I,III, Spannw. und Flügel) . . 87

5.20 Nomenklatur der abgelösten Strömung auf dem Doppelrampenmodell . . . 89

5.21 Sichtbarmachung der Doppelrampenströmung (Bed. III) . . . . . . . . . . . 91

5.22 Sichtbarmachung der Doppelrampenströmung (Bed. I) . . . . . . . . . . . . 91

5.23 Entwicklung von Druck und Wärmestromdichte auf der Doppelrampe (Bed. III) 92

5.24 Entwicklung von Druck und Wärmestromdichte auf der Doppelrampe (Bed. I) 93

5.25 Zeitliche Entwicklung der Ablöselänge für Bedingung III und I . . . . . . . . 94

5.26 Messung der Ablösung für Versuch $453 \ldots \ldots$. . . . . . . . . . . . 95

5.27 Fehlerbestimmung der gemessenen Ablöselänge . . . . . . . . . . . . . . 96

5.28 Normierte Druckmessungen für alle Doppelrampenversuche . . . . . . . . . 97

5.29 Normierte Wärmestromdichtemessungen für alle Doppelrampenversuche . . 98

5.30 Normierte Ablöselänge in Abhängigkeit von $\Theta_{W}$ und $R e_{x 1} \ldots \ldots$. . . . . . 100

5.31 Druckverhältnis für beginnende Ablösung und Korrelation von Needham . . 101

5.32 Wärmstromverhältnis zum Druckverhältnis beim Wiederanlegen . . . . . . . 102

6.1 Entwurf eines Doppelrampenmodells . . . . . . . . . . . . . . . . . . . . 104 
A.1 Schema der Kontaktkalibrierung für Thermoelemente . . . . . . . . . . . . 125

A.2 Photographische Ansicht der Thermoelementkalibrierung . . . . . . . . . . 126

A.3 Ergebnis der Thermoelementkalibrierung . . . . . . . . . . . . . . . . 127

B.1 Fehler bei der Messung der Wärmestromdichte . . . . . . . . . . . . . . . . 130 



\section{Tabellen}

2.1 Erforderliche Kollisionen, um Gleichgewicht zu erreichen . . . . . . . . . . . 11

3.1 Technische Daten des HEG . . . . . . . . . . . . . . . . . . . . . . . . . . 22

3.2 Übersicht der durchgeführten HEG-Experimente . . . . . . . . . . . . . . . . . . . . . . . . . . . . 26

3.3 Messzeitfenster für alle Versuche . . . . . . . . . . . . . . . . . . . . . . . . . . . 27

3.4 Messung der Stoßgeschwindigkeit . . . . . . . . . . . . . . . . . . . . . . . . 28

3.5 Stoßgeschwindigkeiten, Berstdrücke und Kanalpositionen für alle Versuche . 31

3.6 Reservoirbedingungen für alle Versuche . . . . . . . . . . . . . . . . . . . . 33

3.7 Messungen der permanenten Sonde . . . . . . . . . . . . . . . . . . . . . . 36

4.1 Gladstone-Dale-Konstanten . . . . . . . . . . . . . . . . . . . . . . . . . . 39

4.2 Optische Auflösung der Hochgeschwindigkeits-Sichtbarmachung . . . . . . 42

4.3 Kenndaten für KULITE-Druckaufnehmer . . . . . . . . . . . . . . . . . . . . 52

4.4 ISO-Kodierung für Thermoelemente . . . . . . . . . . . . . . . . . . . . . . . . . . . . . . . . . . . . . . 54

4.5 Stoffwerte der Thermoelemente . . . . . . . . . . . . . . . . . . . . . . . 56

4.6 Koordinaten der Sensoren des X-38-Modells . . . . . . . . . . . . . . . . . 65

5.1 Zylinderversuche . . . . . . . . . . . . . . . . . . . . . . . . . 67

5.2 Vergleich der Zylinder-Staupunktdrücke zwischen Experiment und Numerik . 68

5.3 Vergleich der Staupunktswärmeströme zwischen Experiment und Numerik . 69

5.4 Vergleich der experimentellen und der numerischen Stoßabstände (3D) . . . 76

5.5 Vergleich der experimentellen und der numerischen Stoßabstände (2D) . . . 76

5.6 Versuche mit dem X-38-Modell . . . . . . . . . . . . . . . . . . . . . . 81

5.7 Übersicht über die durchgeführten Doppelrampenversuche . . . . . . . . . 90

5.8 Wertetabelle für die Doppelrampenversuche . . . . . . . . . . . . . . . . . 99

5.9 Ablösung nach Katzer . . . . . . . . . . . . . . . . . . . . . . . . . . . . . . 100

5.10 Wertetabelle für die Korrelation nach Needham . . . . . . . . . . . . . . . . 101 



\section{Nomenklatur}

\section{Kennzahlen}

$$
\begin{aligned}
& C \text { : Chapman-Rubesin-Parameter } \\
& \text { Da : Damköhlerzahl } \\
& M \text { : Machzahl } \\
& K n \text { : Knudsenzahl } \\
& \text { Re : Reynoldszahl }
\end{aligned}
$$

\section{Griechische Symbole}

$\begin{array}{llll}\alpha & : & \circ & \text { Anstellwinkel } \\ \beta & : & & \text { Skalierungsfaktor } \\ \Delta & : & \mathrm{m},{ }^{\circ} & \text { Stoßabstand, Schiebewinkel } \\ \lambda & : & \mathrm{m} & \text { mittlere freie Weglänge } \\ \mu & : & \frac{N s}{m^{2}} & \text { dynamische Viskosität } \\ \omega & : & \frac{1}{s} & \text { Kreisfrequenz } \\ \Theta_{1} & : & \circ & \text { Anstellwinkel der ersten Rampe bzgl. der Anströmrichtung } \\ \Theta_{2} & : & \circ & \text { Anstellwinkel der zweiten Rampe bzgl. der Anströmrichtung } \\ \Theta_{W} & : & \circ & \text { Anstellwinkel der zweiten Rampe bzgl. der ersten Rampe } \\ \phi & : & & \text { Phasenverschiebung } \\ \varphi & : & \circ & \text { Clekel } \\ \Psi & : & \frac{V}{m} & \text { Dichte } \\ \rho & : & \frac{k g}{m^{3}} & \text { Standardabche Feldstärke } \\ \sigma & : & \sqrt{\frac{1}{N} \sum_{i=1}^{N}\left(x_{i}-\bar{x}\right)^{2}} & \text { Zeit } \\ \tau & : & s & \text { Partialdichte }\end{array}$




\section{Lateinische Symbole}

\begin{tabular}{|c|c|c|}
\hline$a$ & $\frac{m^{2}}{s}, \frac{m}{s}$ & $\begin{array}{l}\text { Temperaturleitfähigkeit/Temperaturleitwert } \\
\text { Schallgeschwindigkeit }\end{array}$ \\
\hline$A$ & - & Amplitude \\
\hline$b$ & $m$ & Länge \\
\hline$B$ & $m$ & Breite \\
\hline$c$ & $\frac{J}{K k g}, \frac{m}{s}$ & spezifische Wärmekapazität, Lichtgeschwindigkeit \\
\hline$D$ & $m$ & Durchmesser \\
\hline$e$ & $J$ & innere Energie \\
\hline$f$ & $m,-$ & Brennweite, Verhältnis \\
\hline$F$ & $m^{2}$ & Fläche \\
\hline$g$ & $\frac{m}{s^{2}}$ & Erdbeschleunigung \\
\hline$h$ & $\frac{J}{k g}$ & Enthalpie \\
\hline$\hbar$ & $1.05457168(18) 10^{-34} J s$ & Wirkungsquantum \\
\hline$H$ & $m$ & Flughöhe \\
\hline$I$ & $l x$ & Beleuchtungsstärke \\
\hline$K$ & $m^{3} / k g$ & Gladstone-Dale-Konstante \\
\hline$k$ & $\frac{W^{\prime}}{K m}$ & Wärmeleitfähigkeit \\
\hline$L$ & $m$ & Länge \\
\hline$m$ & $k g,-$ & Masse, Phasenkontrast \\
\hline$n$ & & Brechungsindex \\
\hline$N$ & & Ordnung \\
\hline$p$ & $P a$ & Druck \\
\hline$\dot{q}$ & $\frac{W}{m^{2}}$ & Wärmestromdichte \\
\hline$r, R$ & $m$ & Radius \\
\hline$t$ & $s$ & Zeit \\
\hline$T$ & $K$ & Temperatur \\
\hline$u$ & $\frac{m}{s}$ & Geschwindigkeit \\
\hline$U$ & $\stackrel{\stackrel{a}{V}}{V}$ & Spannung \\
\hline$w$ & $\frac{k g}{m^{3} s}$ & Produktionsrate \\
\hline$W$ & $\frac{k g}{m^{2} s}$ & Wellenwiderstand \\
\hline$x, y, z$ & $m$ & Ortskoordinate, Länge \\
\hline
\end{tabular}




\section{Indizes}

0 : Ruhegröße, Lichtgeschwindigkeit im Vakuum

1 : Zustand stromauf eines Stoßes, Füllzustand des Stoßrohres

2 : Zustand stromab eines Stoßes, Zustand hinter dem laufenden Verdichtungsstoß

3 : Zustand hinter der Kontaktfläche

4 : Füllzustand des Kompressionsrohres

$5 \quad$ : Zustand hinter dem reflektierten Verdichtungsstoß

$\infty \quad$ : Anströmgröße

$+\quad$ : Verdrängungsdicke

$G \quad$ : Gegenstandswelle

$B \quad$ : Bezugswelle

$R \quad$ : Rekonstruktionswelle

inc : beginnende Ablösung (engl.: incipient)

$P P \quad$ : permanente Sonde (engl.: permanent probe)

Rot : Rotation

$S$ : Staupunkt,Sensor

Schw : Schwingung

Sep : Ablösung

$W \quad$ : Wiederanlegen

wall : Wand (engl.: wall)

$t$ : Totalwert, Pitot

$\operatorname{Tr} \quad$ : Translation 



\section{Abkürzungen}

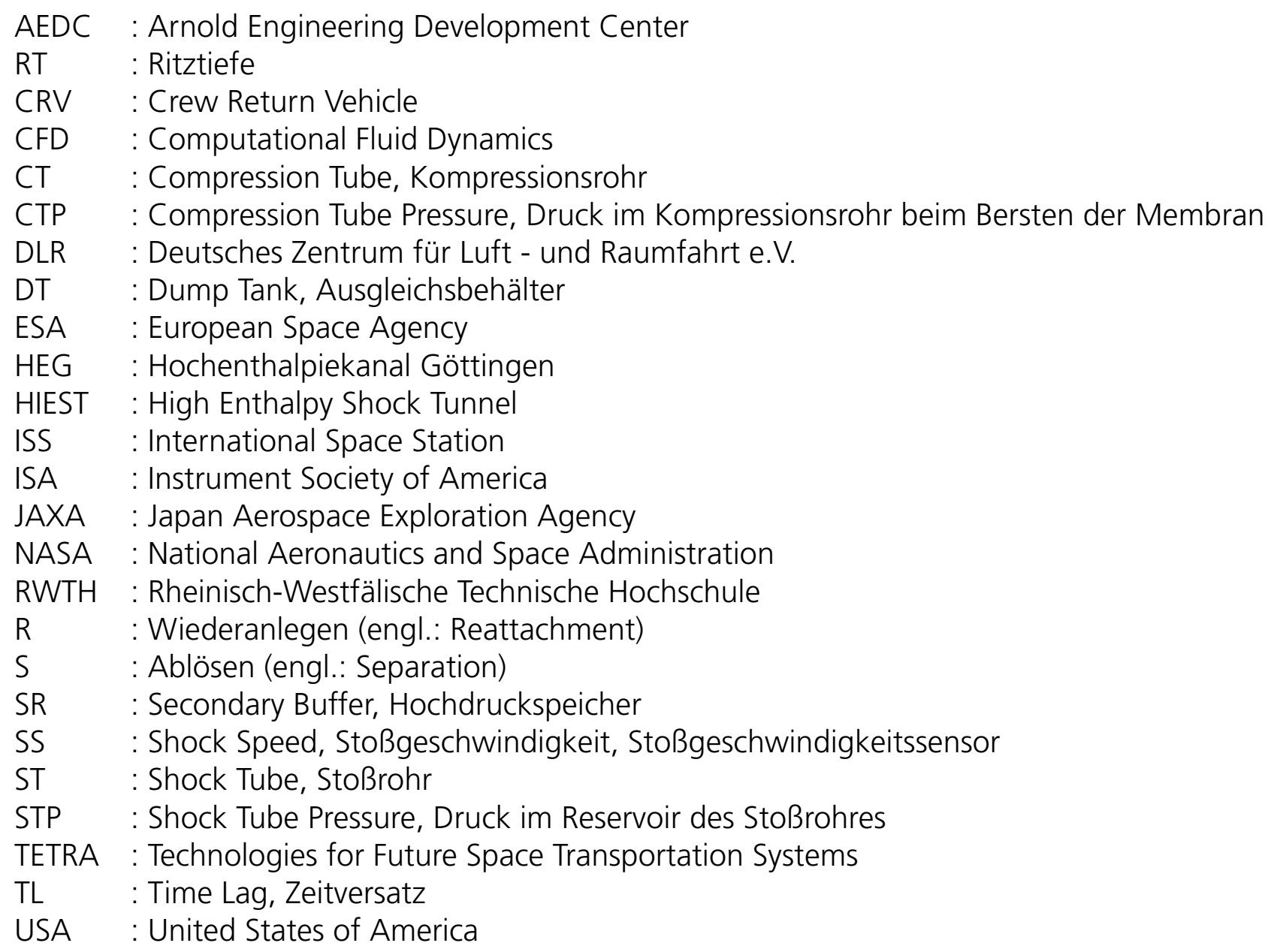





\section{Einleitung}

\subsection{Hintergrund und Motivation}

Ein Raumfahrzeug besitzt beim Wiedereintritt in die Erdatmosphäre eine hohe Fluggeschwindigkeit und damit eine hohe kinetische Energie. Vor dem Körper des Raumfahrzeuges wird die in der Atmosphäre enthaltene Luft aufgestaut, und die Umwandlung der kinetischen Energie des Raumfahrzeuges erfolgt überwiegend in innere Energie des Gases. Dieser Vorgang führt zu starker Erwärmung der Luft und somit auch zu einer aerodynamischen Erwärmung der Oberfläche des Raumfahrzeuges. Für die Auslegung des Raumfahrzeuges hat diese Erwärmung eine zentrale Bedeutung.

Um in eine Umlaufbahn einzuschwenken, benötigt ein Raumfahrzeug mindestens die erste kosmische Geschwindigkeit ${ }^{1}$ oder Kreisbahngeschwindigkeit, die sich durch das Gleichsetzen von Gravitationskraft und Zentripetalkraft zu

$$
m g=\frac{m u_{\infty}^{2}}{R+H} \Longrightarrow u_{\infty}=7.9 \mathrm{~km} / \mathrm{s}
$$

bestimmen lässt. Hierbei wurde der Erdradius als sehr viel größer als die Flughöhe angenommen $(R \gg H) ; m$ ist die Masse des Raumfahrzeuges und $g$ die Erdbeschleunigung. Mit dieser Geschwindigkeit wird das Raumfahrzeug bei der Rückkehr in die Erdatmosphäre eintreten. Die Temperaturen der atmosphärischen Luft im zu berücksichtigenden Höhenbereich liegen zwischen $180 \mathrm{~K}$ und $300 \mathrm{~K}$. Damit beträgt die Fluggeschwindigkeit eines Raumfahrzeuges ein Vielfaches der Schallgeschwindigkeit der durchflogenen Atmosphäre.

Die Abbremsung des Gases vor dem Raumfahrzeug erfolgt in dieser Überschallströmung über einen Verdichtungsstoß, der für eine Änderung der Zustandsgrößen des Gases verantwortlich ist. Innerhalb dieses Stoßes herrschen sehr starke Gradienten der Zustandsgrößen des Gases. Die Geschwindigkeit des Gases relativ zum Körper sinkt schlagartig, gleichzeitig steigen Dichte, Temperatur und Druck erheblich an. Die Dicke dieses Verdichtungsstoßes ist für Luft in der Größenordnung von $10 \lambda$ (Anderson (1989)), wobei $\lambda$ die mittlere freie Weglänge zwischen zwei Molekülstößen angibt. Bei einer Flughöhe von 70 km entspricht dies $2 \mathrm{~mm}$. Diese Abmessung ist klein gegenüber einer typischen Raumfahrzeuglänge von mehreren Metern, deshalb werden in der Überschallaerodynamik des Wiedereintrittsfluges diese Verdichtungsstöße als Diskontinuitäten behandelt.

Die innere Energie des Gases hinter dem Verdichtungsstoß, dicht vor der Oberfläche des Raumfahrzeuges, ist ausreichend groß, um die Anregung der Schwingungsfreiheitsgrade der Moleküle, ihre Dissoziation und ihre Ionisation zu bewirken. In Abb. 1.1 sind diese Hochtemperatureffekte dargestellt. Die allgemeine Behandlung dieser Phänomene wird

\footnotetext{
${ }^{1}$ Aus dem russischen Sprachgebrauch. Die zweite kosmische Geschwindigkeit ist erforderlich, um das Gravitationsfeld eines Planeten zu verlassen; die dritte, um das Sonnensystem, und die vierte, um die Galaxis zu verlassen.
} 

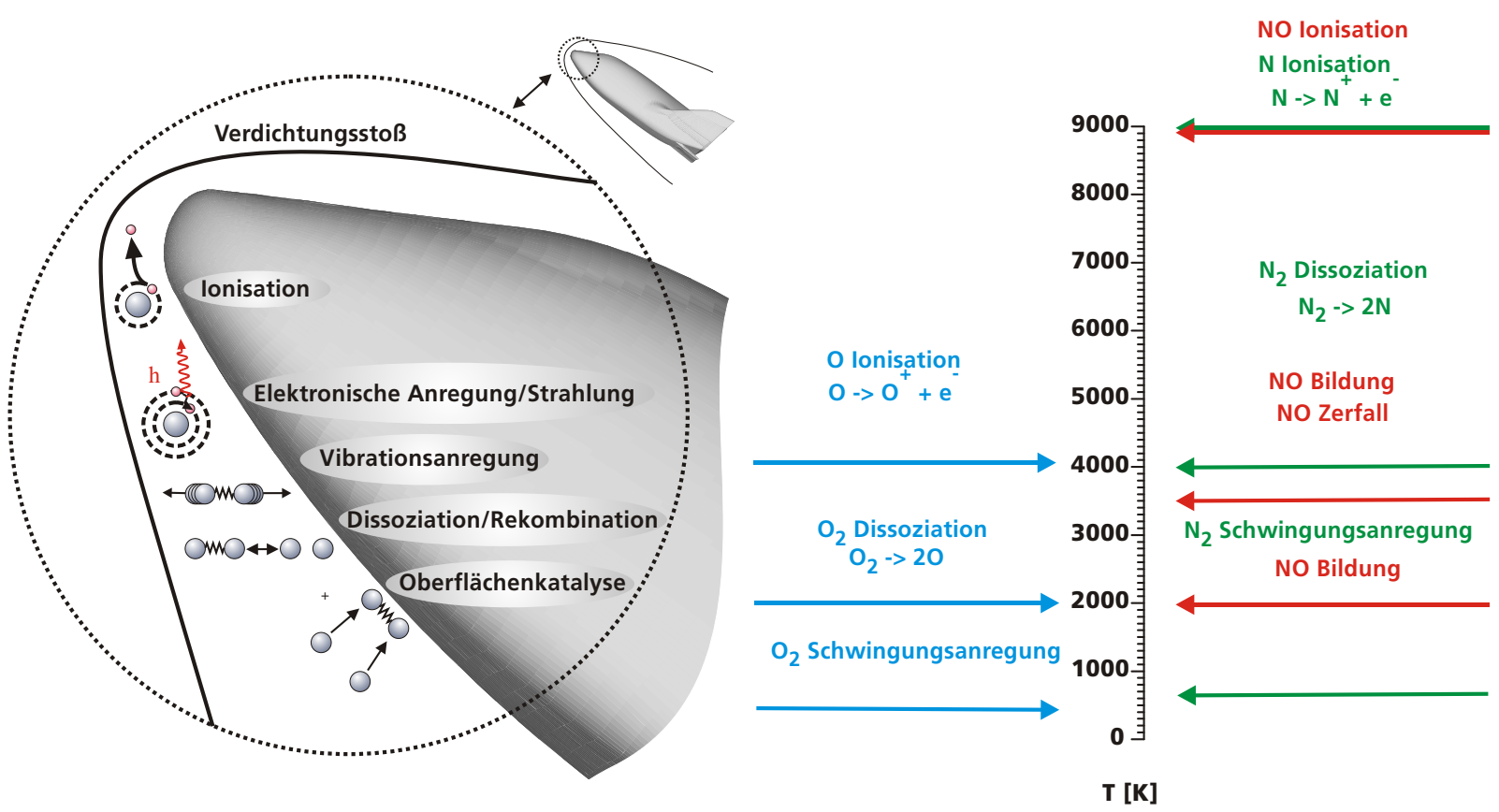

Abbildung 1.1: Darstellung der Hochtemperatureffekte hinter der Bug-Stoßwelle eines Raumfahrzeuges.

als Hochtemperaturgasdynamik oder Gasdynamik unter dem Einfluss von Realgaseffekten² bezeichnet.

Laufen die chemischen und thermischen Zustandsänderungen in Zeiträumen $\tau$ ab, die klein sind gegenüber der Zeit $t_{L}$, die ein Fluidelement braucht, um sich durch das Strömungsfeld um das Raumfahrzeug zu bewegen, befindet sich die gesamte Strömung im Gleichgewicht. Für die diesen Zustand beschreibende Damköhlerzahl gilt dann $D a=t_{L} / \tau \rightarrow \infty$. Bei den hohen Fluggeschwindigkeiten, die während eines Wiedereintrittes herrschen, kann die charakteristische Aufenthaltszeit eines Fluidelementes im Strömungsfeld in die Größenordnung der Reaktionszeiten fallen $(D a=O(1)$ ). Die Strömung befindet sich im Nichtgleichgewicht, Relaxationserscheinungen treten auf. Mit Hilfe der Nichtgleichgewichtsthermodynamik werden diese Relaxationserscheinungen beschrieben. An dieser Stelle sei angemerkt, dass die absolute Strömungsgeschwindigkeit ein Maß für das Auftreten der Phänomene der Hochtemperaturgasdynamik bildet. Um diese Klasse von Strömungen von den allgemeinen Überschallströmungen ${ }^{3}$ zu unterscheiden, benutzt man im englischen Sprachgebrauch den Begriff hypervelocity flow.

${ }^{2}$ Der Ausdruck Realgaseffekte hat sich um 1950 unter den Aerodynamikern etabliert, als offensichtlich wurde, dass bei der Behandlung von Wiedereintrittsströmungen Vibrationsanregung, Dissoziation und lonisation berücksichtigt werden müssen. Hier bezieht sich der Ausdruck auf diese in der Realität auftretenden Hochtemperatureffekte; sie sind nicht gleichzusetzen mit den Effekten, die bei der Behandlung dichter Gase (realer Gase) auftreten (Anderson (1989), Seite 391).

${ }^{3}$ Eine hohe Machzahl M der Strömung, gerade bei der Strömungssimulation im Windkanal, bedingt nicht immer eine hohe Absolutgeschwindigkeit. Niedrige Temperaturen des Gases der freien Anströmung, wie sie oft in kontinuierlich betriebenen Windkanälen auftreten, erlauben eine hohe Machzahl auch bei niedrigen Absolutgeschwindigkeiten der Strömung. 

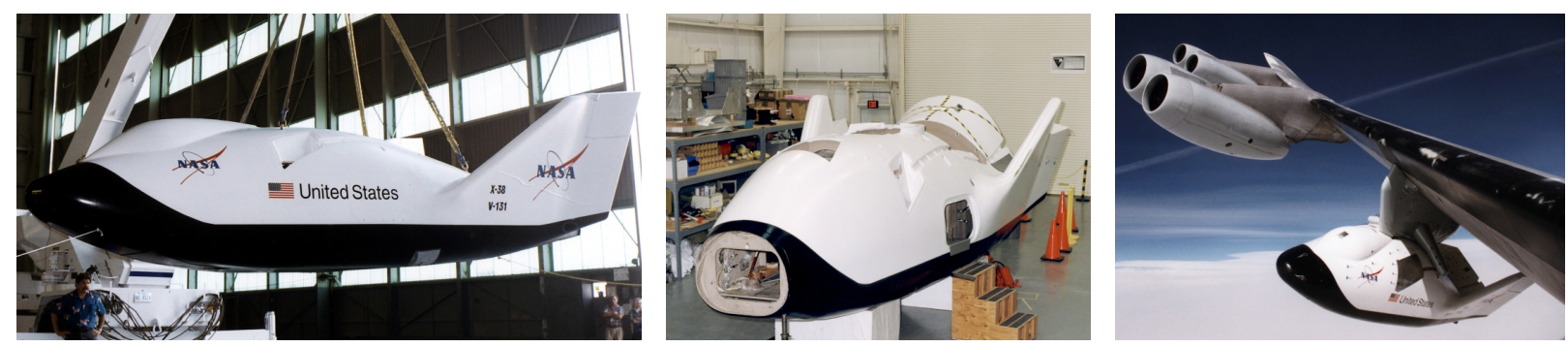

Abbildung 1.2: Photographische Ansichten des X-38 (V131) in der Werkshalle des Dryden Flight Research Center der NASA (links und Mitte) und an der Abwurfeinrichtung einer Boeing B-52 vor seinem atmosphärischen Testflug.

Ein Beispiel für den Einfluss von Hochtemperatureffekten auf die Umströmung einer Widereintrittskonfiguration ist die Nickmoment-Anomalie, die während des ersten Fluges des Space Shuttle beobachtet wurde. Während der Flugphase bei einer Machzahl $M>10$ und einem Anstellwinkel $\alpha>35^{\circ}$ war der benötigte Klappenausschlag für einen getrimmten Flug etwa doppelt so hoch wie die Vorhersagen. Woods u. a. (1983) zeigten, dass ungefähr die Hälfte des Unterschiedes darauf zurückzuführen ist, dass dieser Flugbereich von laminarer Strömung charakterisiert war, während die Vorhersage eine turbulente Wandgrenzschicht annahm. Diese Annahme resultierte in einer zu kleinen Ablöseblase auf der Steuerklappe. Die verbleibende Differenz lässt sich auf den Einfluss der Hochtemperatureffekte auf die Druckverteilung am Körper zurückführen (Griffith u. a. (1983), Maus u.a. (1984)). Die Einflüsse, die Hochtemperatureffekte auf abgelöste Strömungen ausüben, sind nicht gut bekannt. Vornehmlich die Wirkung auf die Ablöselänge und die Wandaufheizung am Wiederanlegepunkt sind hier von Interesse.

Ein Raumfahrzeug, das der Belastung durch die skizzierte Strömung ausgesetzt ist, ist der Technologiedemonstrator $\mathbf{X}-\mathbf{3 8}$, der in Abb. $\mathbf{1 . 2}^{4}$ dargestellt ist. Das X-38-Programm war eine gemeinsame Unternehmung der amerikanischen Raumfahrtbehörde NASA (U.S. National Aeronautics and Space Administration) und der europäischen Raumfahrtagentur ESA (European Space Agency) unter der Führung des NASA Johnson Space Center. Das Ziel dieses Programms war die Untersuchung und Erprobung der wichtigsten Technologien, die zur Entwicklung des zukünftigen Rettungsvehikels CRV (Crew Return Vehicle) für die internationale Raumstation ISS (International Space Station) führen sollen.

Das CRV wird im Notfall die Möglichkeit bieten, alle auf der Raumstation arbeitenden Astronauten auf die Erdoberfläche zurückzuführen. Innerhalb des X-38-Programms sollen alle Untersuchungen mit Hilfe eines so genannten Technologiedemonstrators (X-38) im realen Flug validiert werden. Die Form des CRV basiert auf den Konzepten der Experimentalprogramme X-23 und X-24A, die von der amerikanischen Luftwaffe (U.S. Air Force) Mitte der sechziger und Anfang der siebziger Jahre entwickelt und geflogen wurden (Horvath u. a. (2000)). Im Gegensatz zu den gegenwärtig genutzten russischen Soyuz-Kapseln bietet das Auftriebskörper-Konzept ${ }^{5}$ des X-38 eine größere Manövrierfähigkeit und Seitenreichweite und damit unter Umständen eine kürzere Rückkehrzeit auf die Erdoberfläche. Des Weiteren bietet das CRV im Gegensatz zu der Soyuz-Kapsel für die Nominalbesetzung (sieben

\footnotetext{
${ }^{4}$ Quelle: http://www.dfrc.nasa.gov

${ }^{5}$ lifting body concept
} 
Astronauten) der ISS Platz. Das Lebenserhaltungssystem des CRV ist für neun Stunden Flug nach Abdocken von der ISS ausgelegt. Der Demonstrator X-38 hat am 12. März 1998 seinen ersten atmosphärischen Testflug nach Abwurf von einer Boeing B-52 absolviert. Der erste unbemannte Wiedereintritt in die Erdatmosphäre war ursprünglich im Februar 2003 geplant (Muratore u. a. (2000)). Die Planungen im Jahr 2004 sahen vor, das CRV im Jahr 2006 voll funktionsfähig an der Raumstation andocken zu lassen. Inzwischen hat die NASA aus Kostengründen und aus politischen Gründen das Programm eingefroren und diesen Wiedereintrittsflug in das Jahr 2014 verschoben (Morring (2004)). Die Planung sieht vor, das CRV mit Hilfe des Space Shuttle oder eines alternativen Trägers in einen ISS-Orbit zu bringen und einen unbemannten antriebslosen Widereintritt zu fliegen. Dieser unbemannte Testflug soll ein komplettes Missionsprofil zur Rettung der ISS-Mannschaft absolvieren. Das CRV ist durch die beiden Ruder, die an den Flügelspitzen angebracht sind, und durch die beiden großen Hauptsteuerflächen am hinteren Ende der Unterseite steuerbar. Die Landung erfolgt mit Hilfe eines steuerbaren Fallschirms auf Kufen. Innerhalb des X-38-Programms basiert die aerodynamische und aerothermodynamische Auslegung des CRV auf enger Kooperation zwischen Windkanalversuchen und numerischer Auslegung mittels CFD (Computational Fluid Dynamics). Die Untersuchungen an dieser Wiedereintrittskonfiguration, die im Rahmen dieser Arbeit im Hochenthalpiekanal Göttingen (HEG) durchgeführt wurden, sind die ersten, die den Hochenthalpiebereich bis $22 \mathrm{MJ} / \mathrm{kg}$ (entspricht einer Fluggeschwindigkeit von $6 \mathrm{~km} / \mathrm{s}$ ) abdecken.

In Abb. 1.3 sind unterschiedliche Wiedereintrittstrajektorien in Abhängigkeit von der Fluggeschwindigkeit und dem binären Skalierungsparameter $\rho L$ aufgetragen. Der binäre Skalierungsparameter ist in dieser Auftragung auf die jeweilige Raumfahrzeuglänge $L$ bezogen und legt damit auch die Flughöhe fest. Auf der rechten Achse ist die Knudsen-Zahl, das Verhältnis von mittlerer freier Weglänge zu Bezugslänge $K n=\lambda / L$, auf den Nasenradius des X-38 ( $r=0.3048 \mathrm{~m}$ ) bezogen. Zusätzlich sind in Abb. 1.3 Betriebspunkte des Hochenthalpiekanals Göttingen (HEG) eingetragen. Die Messungen, die in dieser Arbeit diskutiert werden, sind in diesem impulsbetriebenen Hochenthalpie-Stoßkanal durchgeführt worden. Zusätzliche Trajektorien sind die des Flugexperimentes SHEFEX (Sharp Edge Flight Experiment) des DLR (Deutsches Zentrum für Luft- und Raumfahrt) (Eggers u.a. (2006)), des Demonstratorkonzeptes Pre-X der französischen Raumfahrtbehörde CNES (Centre National d'Etudes Spatiales) (Chavagnac u. a. (2003)) und die Rückkehrbahn der Apollo 11-Kapsel der NASA nach der ersten Mondlandung (Manders (1970)).

Beim Wiedereintritt durchfliegt das X-38 mehrere Strömungsbereiche. Dem freimolekularen Bereich $(K n>10)$ folgt ein Übergangsgebiet $(0.01<K n<0.1)$ in der Thermosphäre zur Kontinuumsströmung $(K n<0.01)$ hin. Ab einer Flughöhe von etwa $95 \mathrm{~km}(K n \approx 0.01)$ über Grund werden Zustandsänderungen des Gases dann durch die kontinuumsmechanischen Gleichungen beschrieben. Im Flugbereich der Kontinuumsströmung findet auch ein Übergang von laminarer zu turbulenter Strömung statt, der großen Einfluss auf die Umströmung des Körpers hat. In der Abb. 1.3 sind diese Bereiche durch eine gestrichelte Linie getrennt, bei der als Transitionsgrenze die Reynolds-Zahl (hier bezogen auf die Länge des X-38) konstant den Wert $10^{6}$ besitzt. Die größten Wärmelasten auf die Oberfläche des Raumfahrzeuges treten typischerweise hier bei einer Fluggeschwindigkeit von ca. $6 \mathrm{~km} / \mathrm{s}$ auf und in einer Flughöhe von 60 km bis 80 km (Hornung (1993)). 

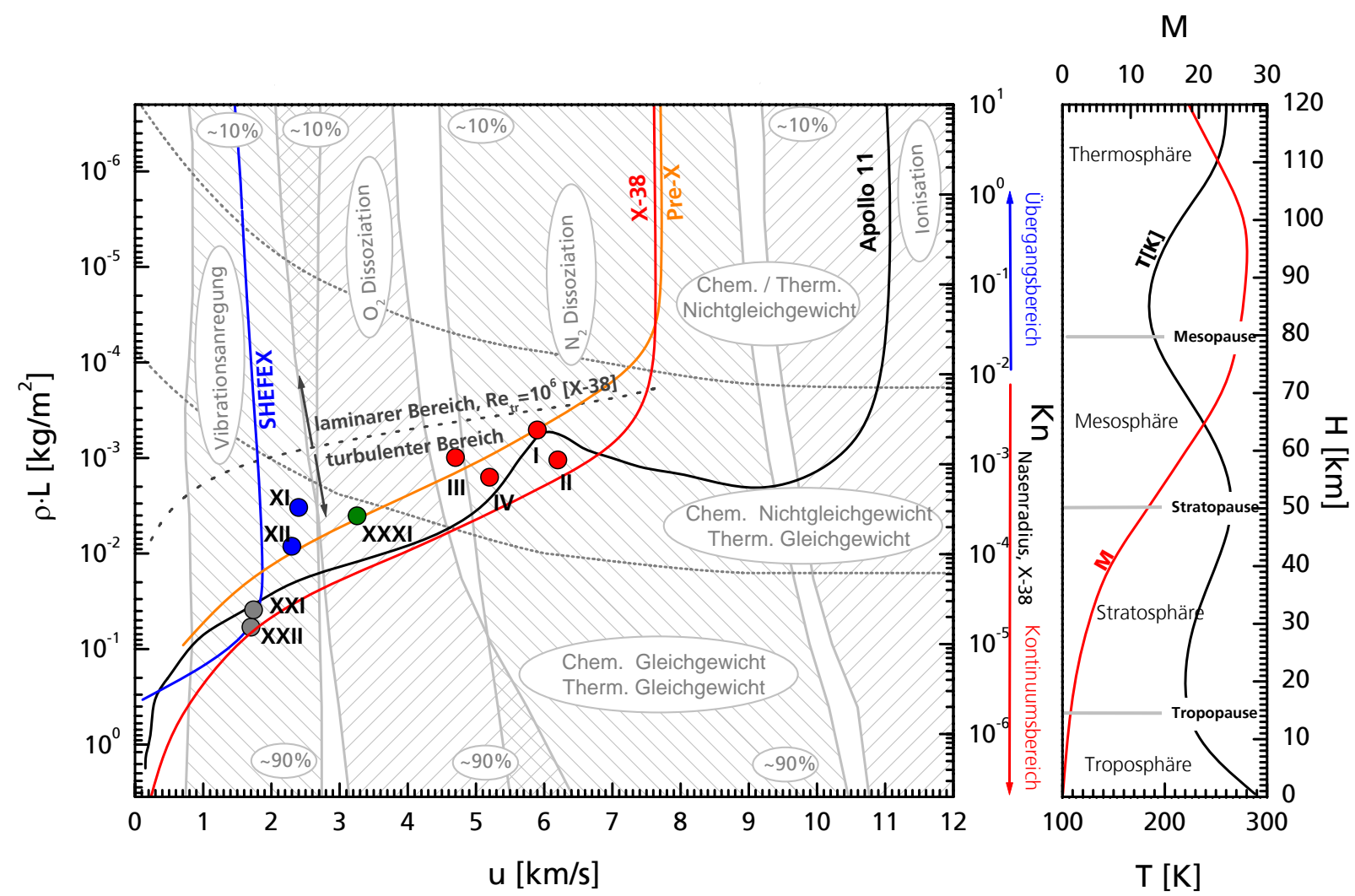

Abbildung 1.3: Darstellung unterschiedlicher Wiedereintrittstrajektorien in die Erdatmosphäre. In Abhängigkeit von Flughöhe und Fluggeschwindigkeit müssen Hochtemperatureffekte berücksichtigt werden.

Entlang der Trajektorie ändert sich auch grundlegend die Anforderung an die Modellierung der Hochtemperatureffekte. Chemisches und thermisches Gleichgewicht/Nichtgleichgewicht sind zu berücksichtigen. Diese Bereiche sind in der Abb. 1.3 durch gepunktete Linien qualitativ getrennt. Neben der Dissoziation und Rekombination treten Vibrationsanregung und bei sehr hohen Fluggeschwindigkeiten auch Ionisation und Strahlung auf. In der Abb. 1.3 sind diese Bereiche schraffiert eingezeichnet, wobei die untere und obere Grenze mit 10\% und $90 \%$ eingetragen sind. In dieser Arbeit wird überwiegend der Einfluss der Dissoziation und Rekombination der Moleküle berücksichtigt. Um die Übertragung der realen Strömungssituation ins Experiment zu vollziehen, müssen die für die Beschreibung des Problems wichtigen Kennzahlen richtig wiedergegeben werden. Die Übertragung aller das Problem beschreibenden Kennzahlen ist im Allgemeinen nicht möglich. Es ist deshalb für die exakte Untersuchung dieser Strömungszustände unerlässlich, Experimente mit numerischen Verfahren zu vergleichen und zu diskutieren. Eine analytische Behandlung ist für das Gesamtströmungsfeld im Allgemeinen schwierig oder nur mit groben Vereinfachungen möglich. 


\subsection{Problemstellung und Zielsetzung}

Die in dieser Arbeit untersuchte Wiedereintrittskonfiguration ist die des Technologiedemonstrators $X-38$. Um die Umströmung der X-38-Konfiguration experimentell zu untersuchen, wurde ein skaliertes Modell im Windkanal vermessen. Ein Windkanal, der in der Lage ist, die benötigten hohen absoluten Strömungsgeschwindigkeiten zu erzeugen, ist der Hochenthalpiekanal Göttingen (HEG) des Instituts für Aerodynamik und Strömungstechnik im Deutschen Zentrum für Luft- und Raumfahrt e.V. (DLR). In diesem Stoßwellenrohr, das mit einem freifliegenden Kolben zur Kompression des Treibgases arbeitet, ist es möglich, die benötigten Strömungsgeschwindigkeiten von $6 \mathrm{~km} / \mathrm{s}$ zu erreichen. Kernpunkt der experimentellen Untersuchungen ist die Umströmung der beiden Hauptsteuerklappen auf der Unterseite des Raumfahrzeuges. Hier wurden beim Vergleich zur numerischen Simulation starke Abweichungen im Bereich der Klappenumströmung gefunden. Die abgelöste Strömung im Bereich der Klappen produzierte beim Wiederanlegen sehr hohe Werte in der Wandwärmestromdichte, die eine numerische Modellierung nicht reproduzieren konnte. Die numerische Simulation nahm ein rein laminares Verhalten der Wandgrenzschicht an. Ziel dieser Arbeit ist es, Einflüsse auf diese Klappenumströmung zu identifizieren und zu klären, wo die Abweichungen ihren Ursprung haben könnten. Bei den Messungen am skalierten Windkanalmodell des X-38 war eine optische Untersuchung der Strömung in der Umgebung der Scharnierlinie nicht möglich, da der direkte Zugang durch die angestellten Klappen blockiert wurde. Weiterhin war die Länge der abgelösten Strömung an der Scharnierlinie der Klappen zu klein, um optische Untersuchungen durchzuführen. Auch ist die Geometrie des X-38-Modells und die sich daraus ergebende Strömungstopologie zu komplex, um vereinfachende Annahmen machen zu können. Die Umströmung der Steuerklappen des Windkanalmodells ist schematisch in der Abb. $\mathbf{1 . 4}$ dargestellt. Die Unterseite der X-38-Konfiguration ist nicht eben, die Klappenscharniere liegen nicht senkrecht zur Blickrichtung. Mit größerem Klappenausschlag bekommen die Klappenflächen einen Winkel zur Symmetrieebene. All dies führt zu einer Dreidimensionalität der Strömung.

Aus diesem Grund wurden Untersuchungen an einer vereinfachten Geometrie durchgeführt. Diese Messungen erfolgten an einem Klappenmodell, das aus zwei im Winkel verstellbaren Rampen besteht. Der optische Zugang ist besser als am X-38-Modell, die abgelöste Strömung durch die Wahl des Modells näher an der zweidimensionalen Vorstellung. Auch ist die numerische Bestimmung der Vorlaufströmung im Bereich reagierender Strömungen auf der einfachen Rampe mit wenig Aufwand möglich, um Zustandsgrößen der erzeugten Vorlaufströmung zu erhalten, die sich nicht experimentell ermitteln lassen. Mit Hilfe dieser Zustandsgrößen lassen sich dann Ähnlichkeitsparameter bilden, die zum Vergleich mit existierenden Korrelationen benötigt werden.

Die Nasenform des X-38, die Einfluss auf diese Parameter hat, ist stumpf, nicht scharfkantig wie beim Rampenmodell. Auch für die stumpfe Nasenform wurde die Umströmung modellbildend im Experiment mittels eines querangeströmten Zylinders erzeugt, und die die Strömung bestimmenden Parameter wurden vermessen. Die Umströmung der Nase des Windkanalmodells bestimmt den weiteren Verlauf der Umströmung der Unterseite des Modells und damit den Strömungszustand, mit dem die Steuerklappe beaufschlagt wird. 


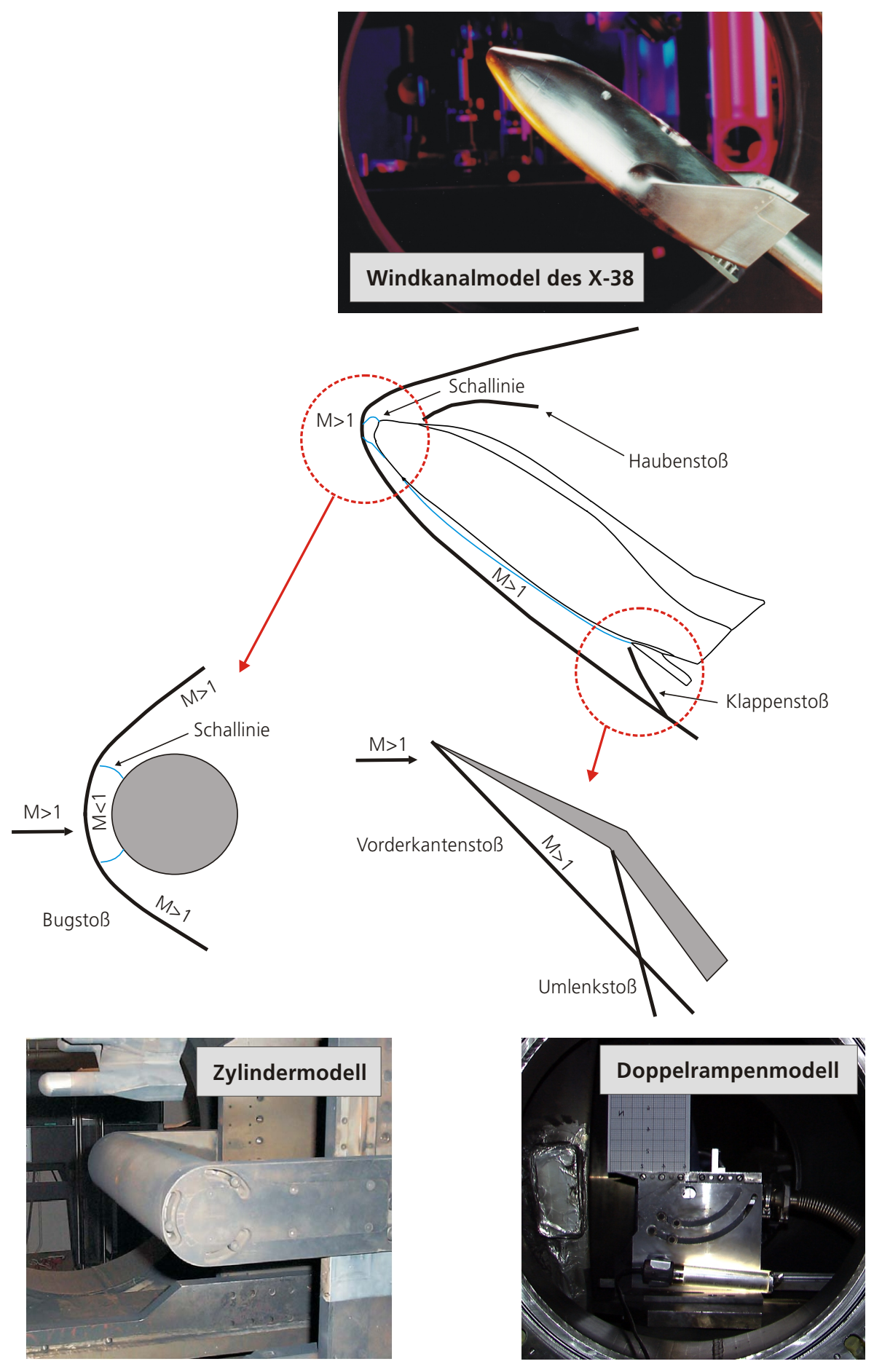

Abbildung 1.4: Schema zur Zerlegung der dreidimensionalen Umströmung eines Windkanalmodells in zweidimensionale Strömung mittels generischer vereinfachter Windkanalmodelle. 


\section{Stand der Forschung}

\subsection{Hochtemperatureffekte}

Bei den beim Wiedereintritt auftretenden hohen Fluggeschwindigkeiten und damit hohen Machzahlen wird, wie schon in der Einleitung skizziert, das Gas im Bereich der Nase eines Raumfahrzeuges stark aufgeheizt. Mit Anwendung des Energiesatzes entlang der Nasenstaustromlinie eines Raumfahrzeuges $\left(h+u_{\infty}^{2} / 2=h_{S}\right)$ kann die folgende Abschätzung (s.a. Anderson (1989), Hirschel (2005), Oertel (1994)) gemacht werden: Die hohe Wiedereintrittsgeschwindigkeit lässt die Vernachlässigung der Enthalpie $h$ gegenüber der kinetischen Energie pro Masse $u_{\infty}^{2} z u$. Für ein ideales Gas gilt dann näherungsweise:

$$
h_{S} \approx \frac{u_{\infty}^{2}}{2}, h_{S}=\frac{7}{2} R T_{S}, a_{\infty}^{2}=\frac{7}{5} R T_{\infty} \Longrightarrow \frac{T_{S}}{T_{\infty}} \approx \frac{M_{\infty}^{2}}{5} .
$$

Für die Temperatur auf der Staustromlinie direkt hinter dem Verdichtungsstoß ergibt sich dann z.B. in $90 \mathrm{~km}$ Flughöhe für das X-38-Vehikel bei einer Flugmachzahl von $M=27 T_{S} \approx$ 26700 K. Dieser Wert stellt eine obere Grenze dar, da das Modell des idealen (kalorisch perfekten) Gases aufgrund der Anregung der inneren Freiheitsgrade der Luftmeloküle für diese Abschätzung nicht mehr gültig ist. In der Realität stellen sich deutlich niedrigere Temperaturen ein. Dieses ist in Abb. 2.1 für die Flugmachzahlen des X-38-Vehikels beim Wiedereintritt dargestellt. Die großen Unterschiede in den Temperaturwerten sind durch die Hochtemperatur- oder Realgaseffekte bedingt. Unter diesen Begriffen werden die Anregung der Schwingung und der Rotation und der elektronischen Freiheitsgrade der Luftmoleküle sowie die gesamten chemischen Prozesse des Gases zusammengefasst.
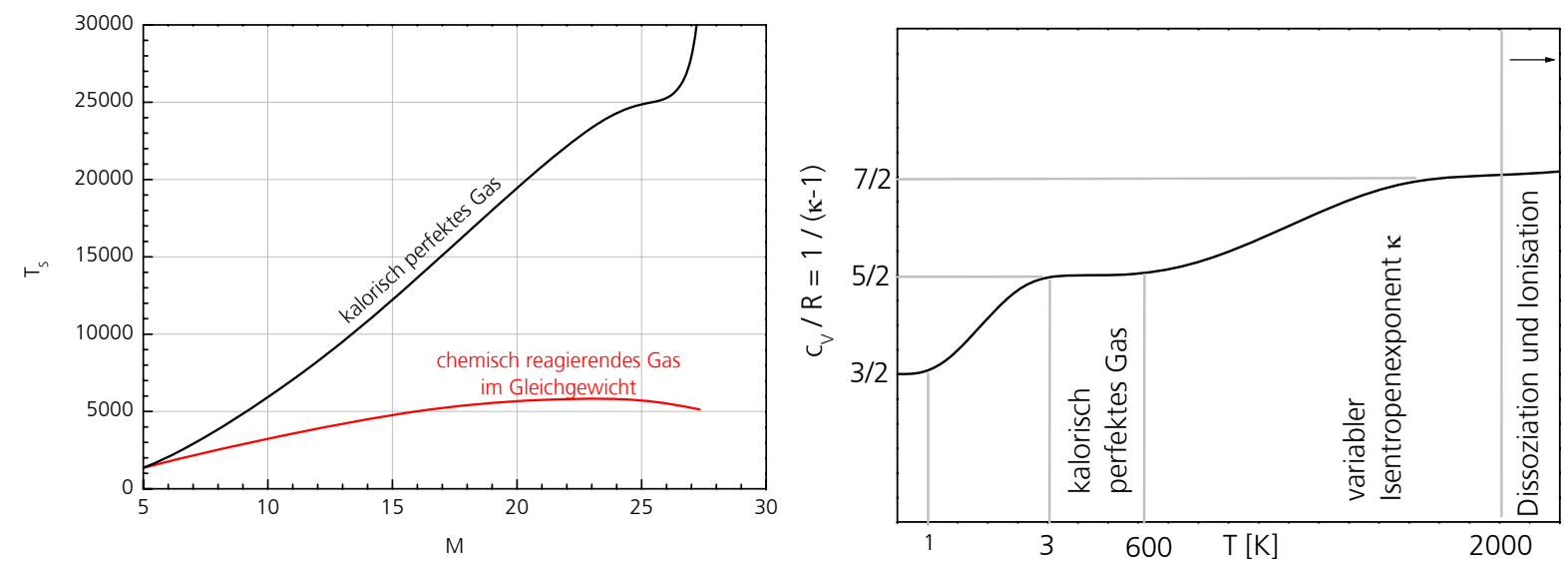

Abbildung 2.1: Temperaturen hinter dem Nasenverdichtungsstoß des $X$-38-Vehikels für ein ideales (kalorisch perfektes) und ein chemisch reagierendes Gas im Gleichgewicht (links). Die Werte des chemisch reagierenden Gases wurden mit den Programm CEA() bestimmt. Temperatureinfluss auf den Isentropenexponenten eines zweiatomigen Gases bei Umgebungsdruck (rechts). 
In der folgenden Diskussion wird die elektronische Anregung nicht berücksichtigt, da sie bei den experimentell erzeugten Strömungszuständen im HEG eine untergeordnete Rolle spielt. Bei Umgebungsbedingungen kann von einem idealen Gas ausgegangen werden, bei dem nur die translatorischen und rotatorischen Zustände angeregt sind. Für dieses kalorisch perfekte Gas sind die spezifischen Wärmekapazitäten $c$ konstant, was zu der Vereinfachung eines konstanten Isentropenexponenten $\kappa=c_{p} / c_{V}$ führt. Die spezifischen Wärmekapazitäten

$$
c_{V} \equiv\left(\frac{\partial e}{\partial T}\right)_{V}=\text { konst. und } c_{p} \equiv\left(\frac{\partial h}{\partial T}\right)_{p}=\text { konst } .
$$

sind als Funktion der Temperatur definiert, und für die spezifische Gaskonstante gilt $\mathrm{R}=c_{p}-$ $c_{V}$. Für die innere Energie eines Gases $e$ und seine Enthalpie $h$ im thermischen Gleichgewicht gilt (Anderson (1989))

$$
e=R \cdot T^{2} \cdot\left(\frac{\partial \ln Q}{\partial T}\right) \text { und } h=e+R \cdot T=e+\frac{p}{\rho},
$$

wobei Q die sogenannte Zustandssumme ist. Die Zustandssumme für ein Molekül setzt sich aus den Zustandssummen der jeweils möglichen Energieanregungen zusammen:

$$
Q=Q_{T r} \cdot Q_{R o t} \cdot Q_{S c h w} .
$$

Mit den Definitionen für die einzelnen Zustandssummen (Vincenti u. Kruger (1975))

$$
Q_{T r}=\left(\frac{4 \cdot \pi \cdot m \cdot k \cdot T_{T r}}{\hbar^{2}}\right)^{3 / 2}, Q_{R o t}=\frac{1}{2}\left(\frac{T_{R o t}}{\Theta_{R o t}}\right) \text { und } Q_{S c h w}=\frac{1}{1-e^{-\Theta_{S c h w} / T_{S c h w}}}
$$

ergibt sich für die spezifische Wärmekapazität eines Moleküls

$$
c_{V}=\underbrace{\frac{3}{2} R}_{\text {Translation }}+\underbrace{R}_{\text {Rotation }}+\underbrace{\left(\frac{\Theta_{\text {Schw }}}{T}\right)^{2} \cdot \frac{e^{\Theta_{\text {Schw }} / T}}{\left(e^{\Theta_{\text {Schw }} / T}-1\right)^{2}} \cdot R}_{\text {Schwingung }},
$$

wobei $\Theta_{\text {Schw. }}$ eine charakteristische Schwingungstemperatur ist. Aus der Gl. 2.1 lässt sich ablesen, dass für ein Gas, bei dem nur die translatorischen und rotatorischen Zustände angeregt sind, keine Temperaturabhängigkeit der spezifischen Wärmekapazität besteht. Für ein zweiatomiges molekulares Gemisch ergibt sich dann ein $\kappa$ von 1.4. Diese Annahme gilt bei Umgebungsdruck bis zu einer Temperatur von ca. $600 \mathrm{~K}$. Für höhere Werte werden die Schwingungszustände angeregt, und die spezifischen Wärmekapazitäten und damit auch der Isentropenexponent sind nicht mehr temperaturunabhängig. Dieses Verhalten ist in der Abb. 2.1 auf der rechten Seite dargestellt. Ab einer Temperatur von ca. 2000 K (s.a. Abb. 
1.1) fängt das Gas dann an, seine chemische Zusammensetzung zu ändern. Bei der Berechnung der Wärmekapazitäten müssen nun die einzelnen Gaskomponenten berücksichtigt werden.

Sind alle angeregten Freiheitsgrade eines Moleküls im Gleichgewicht, so dass nur eine einzige Temperatur $T=T_{T r}=T_{R o t}=T_{S c h w}$ existiert, spricht man vom thermischen Gleichgewicht oder vom thermisch perfekten Gas. Während ein kalorisch perfektes Gas immer thermisch perfekt ist, gilt die Umkehrung nicht immer. Ein Gas kann thermisch perfekt sein, obwohl seine Schwingungsfreiheitsgrade angeregt sind. Der Anteil von atomarem Sauerstoff und Stickstoff ist für Luft als kalorisch perfektes Gas gleich null. Hohe Temperaturen und Dichten des Gases können zu einer Verschiebung des chemischen Gleichgewichtszustandes führen. Steigen diese Zustandsgrößen über eine bestimmte Schwelle, beginnen sich die Moleküle in ihre atomaren Bestandteile zu zerlegen. Diese Dissoziationsreaktionen sind in Abhängigkeit von der Temperatur in Abb. 1.1 aufgetragen. Die chemische Zusammensetzung eines Gases ist eine feste Funktion der Zustandsgrößen Dichte und Temperatur. Wenn ein Gas seine Zusammensetzung nicht ändert, befindet es sich im chemischen Gleichgewicht. Findet eine Veränderung der Zusammensetzung statt, benötigt das Gas eine charakteristische Zeit, um seinen thermischen und chemischen Gleichgewichtszustand zu erreichen. Für die Zeitdauer dieser Relaxation befindet sich das Gas in einem Zwischenzustand, dem sogenannten Nichtgleichgewicht. Die Zeit, die von den Molekülen benötigt wird, um ihr thermodynamisches Gleichgewicht zu erreichen, hängt von der Möglichkeit für die Teilchen ab, untereinander zu kollidieren. Die Anzahl der Kollisionen zwischen Molekülen hängt von der Dichte und der Temperatur ab. Die Kollisionsanzahl, bei der Moleküle reagieren, ist viel niedriger als die Anzahl der Kollisionen, die sie ausführen, da nur ein Bruchteil der Kollisionen genug Energie beinhaltet und hiervon nur ein Bruchteil tatsächlich zu einer Anregung führt.

\section{Anregung}

Anzahl der Kollisionen

\section{Translation}

$\mathrm{O}(10)$

\section{Rotation}

$\mathrm{O}(10)$

\section{Schwingung}

$\mathrm{O}\left(10^{4}\right)$
Dissoziation

$$
>\mathrm{O}\left(10^{4}\right)
$$

Tabelle 2.1: Erforderliche Kollisionen, um Gleichgewicht bzgl. der Anregung zu erhalten. Entnommen aus Hirschel (2005).

Aus Tab. 2.1 wird ersichtlich, dass nur wenig Kollisionen erforderlich sind, um eine vollständige Anregung der Translation und Rotation zu erhalten, während die Anregung der Schwingung signifikant mehr Kollisionen benötigt. Für die Dissoziation liegt die Anzahl noch höher. Der Vergleich der charakteristischen Reaktionszeit $\tau$, innerhalb der diese Kollisionen ausgeführt werden, mit einer charakteristischen Aufenthaltszeit eines Fluidelementes im Strömungsfeld $t_{L}=L / u$ führt zur Definiton der schon in der Einleitung erwähnten Damköhlerzahl $D a=t_{L} / \tau$. Diese enthält die folgenden Grenzfälle:

$D a \rightarrow \infty$ : Die Aufenthaltszeit ist viel größer als die charakteristische Reaktionszeit. Der gesamte thermo-chemische Prozess ist im Gleichgewicht; die Strömung wird als Gleichgewichtsströmung bezeichnet.

$D a \rightarrow 0$ : Die Aufenthaltszeit ist viel kleiner als die charakteristische Reaktionszeit. Der gesamte thermo-chemische Prozess ist "eingefroren"; die Strömung wird als gefroren bezeichnet. 
$D a=O(1)$ Die Aufenthaltszeit ist in der Größenordnung der charakteristischen Reaktionszeit. Die thermo-chemischen Prozesse laufen den Strömungsänderungen hinterher: sie relaxieren; die Strömung wird als Nichtgleichgewichtsströmung bezeichnet.

Die Damköhlerzahl kann nicht global für ein Strömungsfeld definiert werden. Selbst wenn sich ein großer Teil der betrachteten Strömung global im Gleichgewicht befindet, kann sich die Strömung z.B. hinter einem Verdichtungsstoß lokal im Nichtgleichgewicht befinden. Die Verwendung einer gemittelten lokalen Damköhlerzahl, wie sie im folgenden Abschnitt definiert wird, kann zu Korrelationszwecken herangezogen werden.

\subsection{Umströmung eines Zylinders bei hohen Geschwindigkeiten}

Grundlegende Arbeiten über die Umströmung von Kugeln und Zylindern in reagierenden Hochenthalpieströmungen stammen von Hornung u. Wen (1995), Hornung (1972) und Hornung (1998). Die aus diesen Arbeiten stammende Herleitung des Zusammenhangs zwischen Stoßabstand und mittlerer Dichte in der Stoßschicht wird an dieser Stelle etwas ausführlicher dargestellt, da sie später zur Diskussion der Ergebnisse der Zylindermessungen herangezogen wird. Der Stoßabstand $\Delta$ ist umgekehrt proportional der mittleren Dichte $\bar{\rho}$ auf der Staustromlinie zwischen Stoß und Wand des Körpers. Abb. $\mathbf{2 . 2}$ zeigt auf der linken Seite den Massenstrom, der durch $b$ in die Stoßschicht eindringt, $\rho_{\infty} u_{\infty} b$. Ist $b$ klein, so ist die Rate, mit der Masse aus dem Kontrollvolumen in Abb. 2.2 entweicht, gleich $u_{\infty} \cos \varphi \bar{\rho} \Delta$. Mit $b=r_{S} \cos \varphi$ ergibt sich beim Gleichsetzen der Massenströme:

$$
\rho_{\infty} u_{\infty} b=u_{\infty} \cos \varphi \bar{\rho} \Delta \Rightarrow \rho_{\infty} u_{\infty} r_{S} \cos \varphi=u_{\infty} \cos \varphi \bar{\rho} \Delta .
$$

Da der Stoßabstand $\Delta$ zum Körper klein gegenüber $r_{S}$ ist, kann $r_{S} \approx r_{Z}$ angenommen werden. Damit ergibt sich:

$$
\rho_{\infty} u_{\infty} r_{Z} \cos \varphi=u_{\infty} \cos \varphi \bar{\rho} \Delta \Rightarrow \frac{\Delta}{r_{Z}} \frac{\bar{\rho}}{\rho_{\infty}}=\hat{\Delta} \frac{\bar{\rho}}{\rho_{\infty}}=1 \Rightarrow \hat{\Delta}=\frac{\rho_{\infty}}{\bar{\rho}} .
$$

Der einfache Zusammenhang zwischen normiertem Stoßabstand und der mittleren Dichte in der Stoßschicht, den Gl. 2.2 wiedergibt, zeigt, dass die Messung des Stoßabstandes auch eine indirekte Messung der mittleren Dichte in der Stoßschicht ist.

In den oben genannten Arbeiten wurde das Reaktionsverhalten der Strömung in der Stoßschicht analytisch untersucht und ein Reaktionsparameter $\tilde{\Omega}$ gefunden, mit dessen Hilfe der Dichteverlauf in der Stoßschicht in Abhängigkeit von der Reaktionsgeschwindigkeit und der Strömungsgeschwindigkeit korreliert werden kann. Die Ergebnisse sind qualitativ in Abb. 2.2 auf der rechten Seite dargestellt. Aufgetragen ist hier die Dichte in der Stoßschicht in zwei Dimensionen, und zwar entlang der radialen Koordinate $r$ und entlang des Umlenkwinkels $\varphi$. Ist die Strömung im Gleichgewicht, zeigt der Dichtesprung über den Stoß das höchste Verhältnis und ist annähernd konstant bis zur Wand. Ist die Strömung hingegen im Nichtgleichgewicht, erreicht das Dichteverhältnis über den Stoß nicht das der Gleichgewichtsströmung. Die Anregung der Translation über den Stoß geschieht sehr schnell, das 

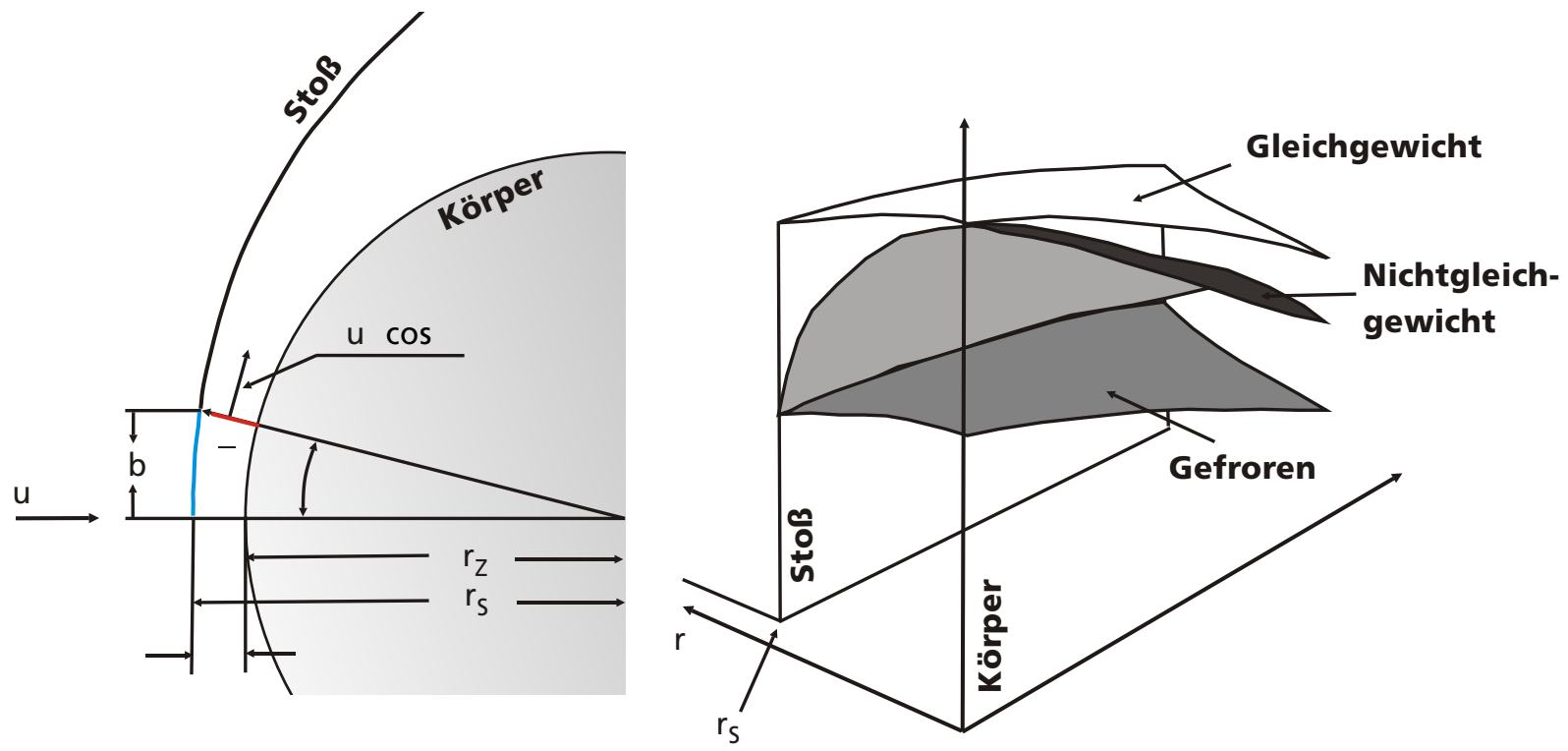

Abbildung 2.2: Topologie der Zylinderumströmung mit der benutzten Notation.

Erreichen des Gleichgewichtes für die Schwingungsfreiheitsgrade nimmt Zeit in Anspruch, darum nimmt die Dichte kontinuierlich bis zur Wand hin zu. Im Fall der eingefrorenen Strömung entspricht das Dichteverhältnis über den Stoß dem Verhältnis der Nichtgleichgewichtslösung und ändert sich auch nicht mehr. Es kann also festgestellt werden, dass eine Messung des Dichteverhältnisses in der Stoßschicht ein direktes Maß zur Validierung der rechnerisch modellierten Reaktionskinetik in der Numerik ist. Der gefundene Reaktionsratenparameter $\tilde{\Omega}$ ist physikalisch das Verhältnis von Energieaustausch durch chemische Reaktionen zum Gesamtenergieeintrag der Anströmung und definiert somit eine mittlere Damköhlerzahl:

$$
D a^{\text {mittel }}=\frac{\Delta \sum_{S} \dot{w}_{s} h_{S}}{\rho_{\infty} u_{\infty}^{3}} .
$$

Die Ergebnisse der in dieser Arbeit vorgestellten Zylinderexperimente werden mit Hilfe dieser Damköhlerzahl diskutiert.

\subsection{Wissensstand über die X-38-Konfiguration}

Der innerhalb des X-38-Projektes geplante Anwendungsbereich für das CRV als Rettungsgefährt wird von Brown (1998) diskutiert. Ein detaillierterer Überblick des X-38-Programmes als in der Einleitung ist bei Asker (1996) zu finden. Der Landeanflug des CRV soll mittels eines steuerbaren Fallschirms durchgeführt werden. Die technische Entwicklung dieses Systems wird von Smith (1997) diskutiert. Die Reduktion des Fahrwerkes auf ein Kufensystem soll zu einer drastischen Reduktion der Entwicklungskosten in diesem Bereich führen. Die 
Hintergründe hierzu werden von Kandebo (1998) und Covault (1998) beleuchtet. Innerhalb des X-38-Programms sind zahlreiche experimentelle und numerische Untersuchungen über die X-38-Konfiguration durchgeführt worden. Hier seien nur kurz die Experimente von Berry u. a. (2001) skizziert, die im Windkanal des NASA Langley Research Center bei einer Machzahl von $M=10$ durchgeführt wurden. Der benutzte Anstellwinkel mit $\alpha=40^{\circ}$ ist identisch dem in dieser Arbeit benutzten Anstellwinkel. Neben der Variation der Reynoldszahl der Anströmung im Bereich von $2 \cdot 10^{6} \mathrm{~m}<\operatorname{Re}<7 \cdot 10^{6} \mathrm{~m}$ wurden Klappenwinkel $\delta$ von $=15^{\circ}, 20^{\circ}$ und $25^{\circ}$ benutzt. Durch die Verwendung von Turbulatoren konnten laminare, transitionelle und vollturbulente Wiederanlegewärmestromdichte auf den Klappen untersucht werden. Die von Berry u. a. (2001) vorgestellten Ergebnisse werden im Abschnitt 5.3 zusammen mit den Experimenten dieser Arbeit diskutiert.

\subsection{Stoß-Grenzschicht-Wechselwirkung}

Bei den in dieser Arbeit betrachteten Strömungsgeschwindigkeiten befindet sich die Strömung in Wandnähe im Überschall, wo ein Druckanstieg immer über einen Verdichtungsstoß erfolgt. Diese Verdichtungsstöße sind von grundlegender Bedeutung für den Widerstand eines Körpers, da sie eine Ablösung der Grenzschicht zur Folge haben können. Die Vorgänge in der Grenzschicht und die Ausbildung des Verdichtungsstoßes beeinflussen sich gegenseitig stark. Diese Wechselwirkung wird für die Stöße stark von der Machzahl bestimmt, während für die Grenzschicht die Reynoldszahl der dominierende Parameter ist. Untersuchungen mit Trennung dieser beiden Einflüsse sind erstmalig von Ackeret u.a. (1946), Liepmann (1946) und Gadd u. a. (1954) durchgeführt worden.

Die systematische Erforschung dieser Wechselwirkung findet seit mehr als 60 Jahren statt. Es ist im Rahmen dieser Arbeit nicht möglich, darüber eine umfassende Darstellung zu geben. Deshalb beschränkt sich dieser Abschnitt auf einen kleinen Überblick der Forschung. Hierbei wird auf das Gebiet der Strömungsablösung im Überschall ohne die Berücksichtigung von Hochtemperatureffekten eingegrenzt. Die Zusammenfassung der Arbeiten, die Hochtemperatureffekte mit einschließen, ist ausführlicher, da hier nicht so viele Untersuchungen vorliegen. Das Hauptaugenmerk liegt auf den Ergebnissen für die Ablöselänge als Parameter für das Steuervermögen einer Kontrollfläche und auf der maximalen Wärmestromdichte beim Wiederanlegen der Strömung als Kriterium für die Materialbelastung. Weitergehende Darstellungen sind bei Holden (2003) und Davis (1999) zu finden.

Infolge der Verdrängungswirkung einer Grenzschicht auf die Außenströmung kommt es zu einer Rückwirkung der Außenströmung auf die Grenzschicht, man spricht von einer Wechselwirkung. Hierbei wird zwischen schwacher und starker Wechselwirkung unterschieden, wobei im Falle der Strömungsablösung eine starke Wechselwirkung vorliegt, die von der Grenzschichttheorie in der ursprünglichen Formulierung von Prandtl nicht erfasst wird. Deshalb existieren Erweiterungen, die diese Grenzschichteffekte höherer Ordnung behandeln. Statt der Einteilung des Strömungsfeldes in Grenzschichtströmung und Außenströmung wird die reibungsbehaftete Grenzschicht noch in zwei Schichten unterteilt und damit eine Dreischichtenstruktur erzeugt, man spricht deshalb von Dreierdeck-Theorie oder asymptot- 
ischer Interaktionstheorie. Eine Übersicht über die Anwendung dieser Grenzschichttheorie höherer Ordnung auf abgelöste Strömungen geben Delery u. Marvin (1975).
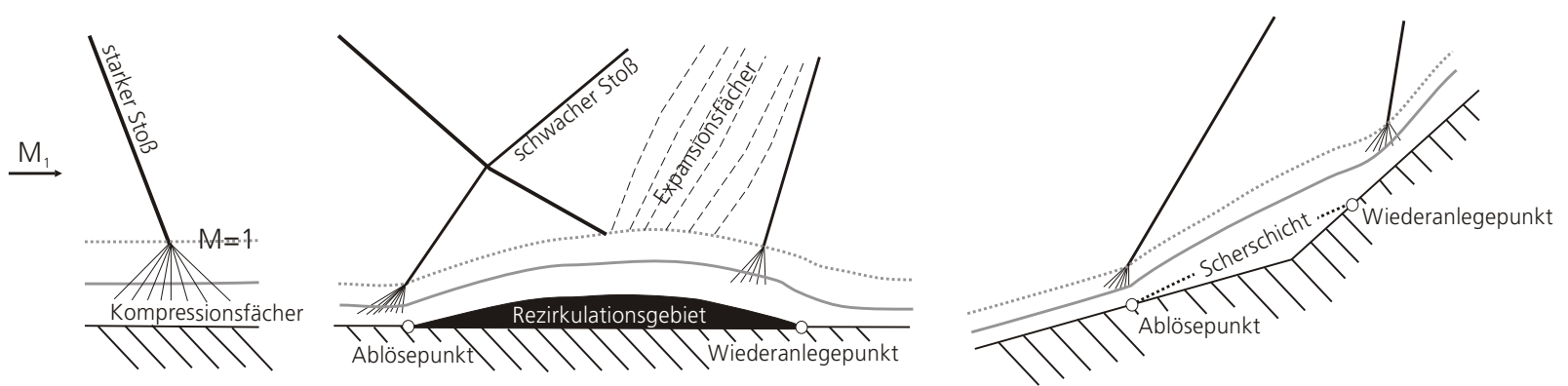

Abbildung 2.3: Topologie einer abgelösten Strömung im Überschall (links) und die Übertragung auf eine schematisierte Steuerklappe eines Raumfahrzeuges.

Trifft im Überschall ein Stoß auf eine Wand, verhindert das wandnahe Unterschallgebiet der Grenzschicht, dass sich der enstandene Stoß bis an die Wand erstreckt. Der Stoß diffundiert, indem er der Grenzschicht einen Druckanstieg aufprägt, der sich in wandnormaler Richtung immer mehr verbreitert, wie in Abb. $\mathbf{2 . 3}$ auf der linken Seite skizziert. Bei laminaren Grenzschichten ist diese Diffusionsbreite mit ca. $100 \delta^{+}$größer als bei turbulenten Strömungen mit $10 \delta^{+}$, wobei $\delta^{+}$die Verdrängungsdicke der Grenzschicht bezeichnet. Dieser Druckanstieg über den Kompressionsfächer wird, wenn hinreichend groß, die Grenzschicht zur Ablösung zwingen. Turbulente Grenzschichten können erheblich größere Druckanstiege als laminare Grenzschichten aufnehmen, da hier die turbulenten Austauschbewegungen kompensierende Wirkung haben.

Ein Beispiel für den experimentellen Aufbau (Bogdonoff u. Kepler (1955)) zur Untersuchung der Wechselwirkung zwischen einem Verdichtungsstoß und einer Grenzschicht in einer Überschallströmung besteht aus einem Keil, der einen Verdichtungsstoß erzeugt, und einer ebenen Wand, auf die dieser Stoß trifft, wie in der Mitte von Abb. 2.3 gezeigt. Bei einem starken Stoß bildet sich bei der Reflexion ein Wellensystem, das aus Verdichtungsund Verdünnungswellen besteht; damit verbunden ist eine Ablösung der Grenzschicht von der Wand. Hinter dem reflektierten Stoß ist die Grenzschicht dicker als vor dem einfallenden Stoß. Wird der Druckanstieg, wie in Abb. 2.3 auf der rechten Seite gezeigt, durch eine Umlenkung der Strömung durch eine Ecke aufgeprägt, löst bei hinreichend großem Umlenkwinkel ${ }^{1}$ auch hier die Grenzschicht ab und bildet ein Rezirkulationsgebiet zwischen der Ablösung und dem Wiederanlegen auf der zweiten Rampe. Die Scherschicht, die das Rezirkulationsgebiet von der Außenströmung trennt, ist in Abb. 2.3 durch die gestrichelte Linie angedeutet. Das Rezirkulationsgebiet wird auch oft als Ablöseblase oder Totwasserregion bezeichnet. Die in Abb. 2.3 gezeigte Doppelrampe dient als vereinfachtes Modell der Steuerklappe eines Raumfahrzeuges.

In Abb. 2.4 ist eine Schlierenaufnahme des sich ausbildenden Strömungsfeldes um das in dieser Arbeit verwendete Klappenmodell zusammen mit der verwendeten Notation dargestellt. Die Wandgrenzschicht löst stromauf der Ecke oder des Scharniers auf der ersten

\footnotetext{
${ }^{1}$ Der Umlenkwinkel bestimmt den Drucksprung.
} 
Rampe ab und legt stromab der Ecke auf der zweiten Rampe wieder an. Zwischen dem Ablösepunkt $S$ und dem Wiederanlegepunkt $R$ der Strömung liegt die Scherschicht $\psi^{*}$, die das Rezirkulationsgebiet von der Außenströmung trennt. Das Gas innerhalb von $\psi^{*}$ verbleibt im Rezirkulationsgebiet, und das Gas außerhalb strömt weiter. Während der Winkel $\Theta_{1}$ zwischen der ersten Rampe und der Anströmrichtung gemessen wird, sind der Ablösewinkel $\Theta_{\text {sep }}$ und der Umlenkwinkel $\Theta_{W}$ der zweiten Rampe in Bezug auf die erste Rampe definiert. Die Ablöselänge $L_{s e p}$ ist die Distanz zwischen dem Ablösepunkt $S$ und dem Wiederanlegepunkt $R$ der Strömung. Der Index $\infty$ bezeichnet das Gebiet der freien Anströmung und der Index 1 die Strömung hinter dem Verdichtungsstoß der Vorderkante am Rande der Grenzschicht kurz vor der Ablösung. Die Indizes 2 und 3 bezeichnen üblicherweise die Regionen hinter dem Ablösestoß und hinter dem Wiederanlegestoß, beziehen sich in der Literatur aber auch oft auf explizit definierte Orte, die je nach Art der Untersuchung (experimentell, analytisch oder numerisch) zugänglich sind.

Die Ablöselänge wird im allgemeinen Fall durch die Parameter bestimmt, die die Grenzschicht vor der Strömungsablösung, die freie Scherschicht und den Druckanstieg beim Wiederanlegen klassifizieren. Dazu gehören die Reynoldszahl $R_{x 1}$ und die Machzahl $M_{1}$ am Grenzschichtrand der Ablösestelle, das Temperaturverhältnis von Wand- zu Gastemperatur am Ablösepunkt $T_{W} / T_{1}$, der Umlenkwinkel $\Theta_{W}$ der zweiten Rampe und das Verhältnis der spezifischen Wärmekapazitäten $\kappa_{1}$. Die Ablöselänge wird außerdem von zwei charakteristischen Längen bestimmt. Diese sind die Grenzschichtdicke $\delta_{1}$ oder die Verdrängungsdicke $\delta_{1}^{+}$und die Lauflänge der Strömung von der Spitze der ersten Rampe bis zum Ablösepunkt $x_{1}$. Unter Berücksichtigung aller aufgeführten Parameter ergibt sich eine Korrelation der Form:

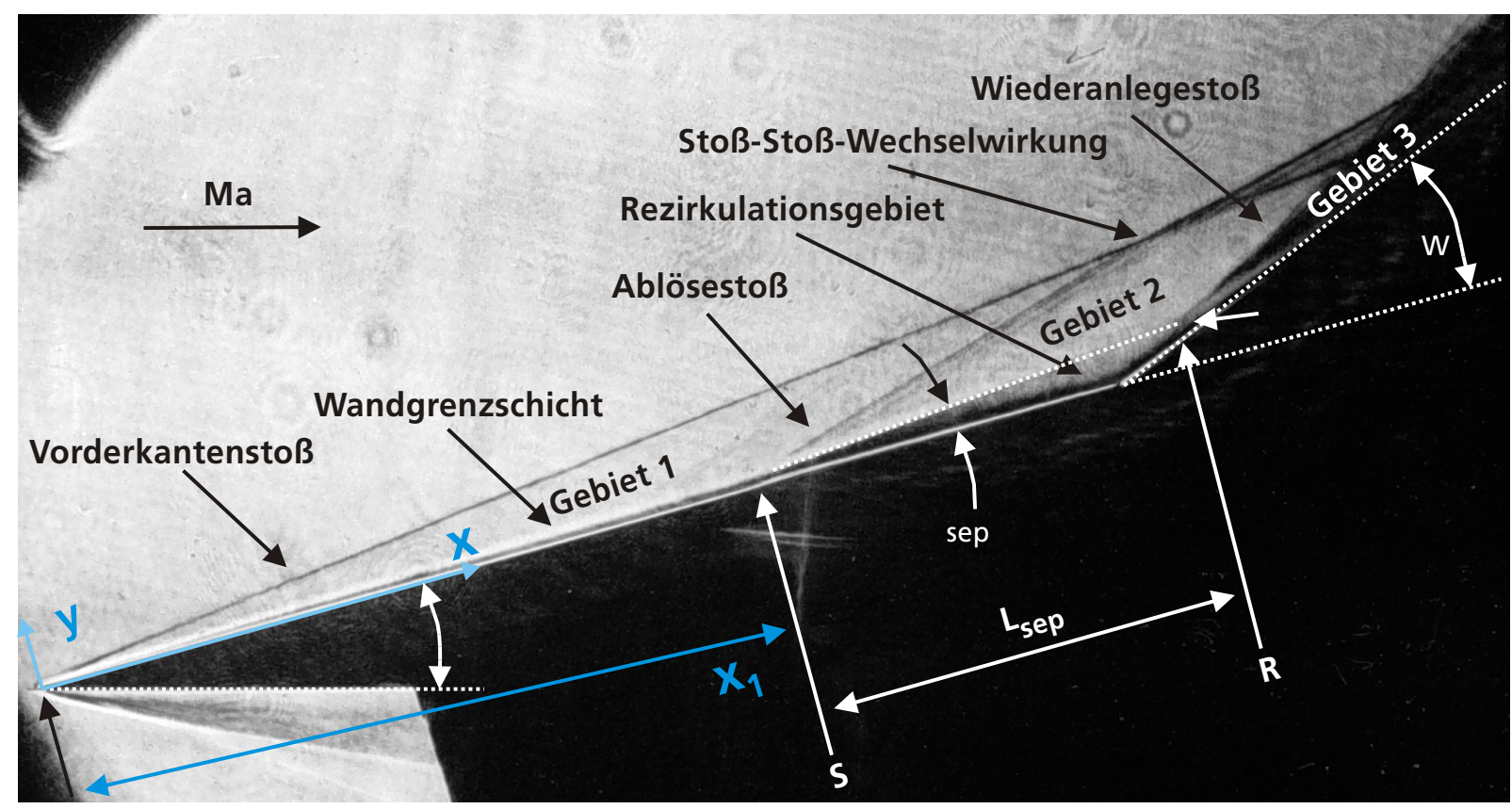

Abbildung 2.4: Umströmung des Rampenmodells. 


$$
\frac{L_{s e p}}{\delta_{1}^{+}}=f\left(\operatorname{Re}_{x 1}, M_{1}, T_{W} / T_{1}, \Theta_{W}, \kappa_{1}\right) .
$$

Ein zentrales Ergebnis zahlreicher numerischer und experimenteller Untersuchungen ist, dass sich die Ablöselänge vergrößert, wenn der Umlenkwinkel $\Theta_{W}$ vergrößert wird, was äquivalent zu einem Druckanstieg beim Wiederanlegepunkt ist. Dieses wurde in vielen experimentellen Arbeiten bestätigt. Dieser Einfluss ist unabhängig davon, ob die Strömung laminar, turbulent oder im transitionellen Bereich ist ( Anders (1970), Bloy u. Georgeff (1974), Coleman u. Stollery (1972), Elfstrom (1972), Ferguson u. Schaefer (1962), Gadd u. a. (1954), Harvey (1968), Hayakawa u. Squire (1982), Holden (1978), Kumar u. Stollery (1994), Miller u. a. (1964), Needham (1965), Settles u. Bogdonoff (1982), Settles u.a. (1979)).

Weiter wurde hinreichend sicher gezeigt, dass auch mit sinkender Machzahl $M_{1}$ die Ablöselänge größer wird ( Anders u. Edwards (1968), Elfstrom (1972), Gadd u. a. (1954), Hayakawa u. Squire (1982), Holden (1971), Holden (1972), Katzer (1989), Miller u. a. (1964), Needham (1965), Roshko u. Thomke (1976)).

Der Einfluss der Wandtemperatur $T_{W}$ auf die Ablöselänge wurde ebenfalls sehr ausführlich untersucht. Hier zeigt sich, dass ein größeres Verhältnis $T_{W} / T_{1}$ die Ablöselänge vergrößert. Eine Kühlung der Wand kann die Ablöselänge also verkleinern. Es zeigt sich, dass dieser Effekt für laminare Strömungen viel stärker ausgeprägt ist als für voll turbulente Strömungen ( Brown u.a. (1990), Curle (1961), Elfstrom (1972), Ferguson u. Schaefer (1962), Georgeff (1974), Holden (1972), Lewis u. a. (1968), Needham (1965) ).

Der Einfluss der Reynoldszahl $R e_{x 1}$ muss nach Strömungsart getrennt betrachtet werden. Für rein laminare Strömungen vergrößert sich die Ablöselänge mit steigender Reynoldszahl $R e_{x 1}$ (Anders u. Edwards (1968), Holden (1971), Johnson (1968), Lewis u. a. (1968), Miller u. a. (1964), Anders u. Edwards (1968), Needham u. Stollery (1966)).

Experimente in transitioneller Strömung zeigen eine Verkleinerung der Ablöselänge mit steigender Reynoldszahl $R e_{x 1}$ (Ferguson u. Schaefer (1962), Gadd u. a. (1954), Johnson (1968), Needham u. Stollery (1966) ).

In voll turbulenter Strömung existieren unterschiedliche Ergebnisse, die vom Reynoldszahlbereich, der untersucht wurde, abhängen. Ergebnisse, die eine Vergrößerung der Ablöselänge mit steigender Reynoldszahl aufweisen, sind von Coleman u. Stollery (1972), Elfstrom (1972) und Holden (1972) publiziert worden. Es gibt aber auch Ergebnisse von Roshko u. Thomke (1976) und Settles u. Bogdonoff (1982), die einen entgegengesetzten Trend gefunden haben. Hunter u. Reeves (1971) fanden bei numerischen Untersuchungen, dass sich die Ablöselänge $L_{\text {sep }}$ mit steigender Reynoldszahl $R e_{x 1}$ vergrößert, wenn die Grenzschicht stromauf der Ablösung umschlägt und hinter der Umlenkung wieder relaminarisiert. Geschieht diese Relaminarisierung erst an der Umlenkung, kehrt sich der Trend um.

Unter den zahlreichen Skalierungen für abgelöste Strömungen werden hier zwei vorgestellt, da sie einen großen Bereich für $M_{1}$ und $R e_{x 1}$ abdecken. Needham (1965) konnte mit 


$$
\frac{L_{s e p}}{x_{1}} \sim \frac{\sqrt{R e_{x 1}}}{M_{1}^{3}}\left(\frac{p_{3}}{p_{2}}\right)^{2}
$$

Messdaten in einem Bereich von $7 \leq M_{1} \leq 15$ und einem weiten Bereich in $\operatorname{Re}_{x 1}$ korrelieren. Bei dieser Untersuchung wurde der Einfluss von $T_{w} / T_{1}$ vernachlässigt. Eine modifizierte Korrelation wurde von Katzer (1989) vorgestellt:

$$
\frac{L_{\text {sep }}}{\delta_{1}^{*}} \sim\left[\frac{\left(T_{W} / T_{1}\right)^{3 / 2}}{1.72+1.11 \cdot\left(\kappa_{1}-1\right) \cdot M_{1}^{2}}\right] \frac{\sqrt{R e_{L} / C}}{M_{1}^{3}}\left(\frac{p_{3}-p_{\text {inc }}}{p_{1}}\right)^{3 / 2} .
$$

Hier ist $p_{\text {inc }}$ der Druck, bei dem Ablösung einsetzt, und ist mit

$$
\frac{p_{\text {inc }}}{p_{1}}=1+\frac{\kappa_{1}}{2} M_{1}^{2} \cdot 1.85 \cdot \sqrt{2 c_{f, L}} \cdot\left(M_{1}^{2}-1\right)^{-0.25}
$$

definiert. Die Verdrängungsdicke $\delta_{1, L}$, Reynoldszahl $R e_{L}$, und Wandschubspannung $c_{f, L}$ als Referenzwerte beziehen sich auf die ungestörte Grenzschicht ohne Ablösung kurz vor der Umlenkung durch die zweite Rampe. Der Einfluss der Wandtemperatur wird durch den Chapman-Rubesin-Parameter $C=\left(\mu_{1} / \mu_{e}\right) /\left(T_{1} / T_{e}\right)$ berücksichtigt. Der Index e steht für den Grenzschichtrand.

Im Bereich sehr hoher Anströmgeschwindigkeiten und Ruheenthalpien $h_{0}$ bis $20 \mathrm{MJ} / \mathrm{kg}$ existieren wenige Untersuchungen. Von Mallinson wurden Untersuchungen durchgeführt (Mallinson u. a. (1992) bis Mallinson u. a. (1997b), ) die keinen Einfluss der hohen Strömungsgeschwindigkeit und damit der Ruheenthalpie auf die Umströmung einer Doppelrampe zeigen. Bei den Untersuchungen einer axialsymmetrischen abstrahierten Space-ShuttleKonfiguration (Hyperboloid Flare) fanden Krek u. a. (1996), dass sich die Ablöselänge bei konstanter Anströmgeschwindigkeit und steigender Ruheenthalpie des Gases verringert. Untersuchungen von Davis (1996) an derselben Geometrie konnten diese Messungen nicht bestätigen. Numerische Untersuchungen von Brenner u. a. (1993) zeigten eine Vekleinerung der Ablöselänge für reagierende Strömungen gegenüber chemisch eingefrorenen Strömungen. Davis führte sehr umfassende Untersuchungen an einer Doppelrampe mit Stickstoff als Versuchsgas durch (Davis u. Sturtevant (1997), Davis (1999), Davis u. Sturtevant (2000)). Hieraus ergab sich eine neue Skalierung basierend auf der Dreierdeck-Theorie, die auch das Temperaturverhältnis $T_{W} / T_{1}$ mit berücksichtigt. Neueste Messergebnisse einer Untersuchung von Bleiblebens u. Olivier (2006), die den Einfluss der Wandtemperatur auf die Ablösung beinhaltete, zeigen große Schwankungen bei der Anwendung der Korrelation von Davis, allerdings gute Übereinstimmung mit Gl. 2.4.

Dieser kurze Überblick über den momentanen Wissensstand über abgelöste Strömungen im Überschall dient als Einführung in die Problematik und wird in seiner Gliederung auf die Auswertung der Messergebnisse mit dem Doppelrampenmodell übertragen. 


\section{Der Hochenthalpiekanal Göttingen}

Der Hochenthalpiekanal Göttingen (HEG) ist ein intermittierend arbeitender Windkanal, in dem Messzeiten im Bereich von Millisekunden realisierbar sind. Die Anlage ist insgesamt $62 \mathrm{~m}$ lang und wiegt 260 t. In Abb. 3.1 sind die wichtigsten Komponenten als Photographie dargestellt. Der HEG wurde im Rahmen des Hermes-Projekts in den Jahren 1990 bis 1992 vom damaligen Konsortium Siemens-Fluidyne gebaut. Der Versuchsbetrieb begann 1993; seitdem wurden in der Anlage insgesamt bis zum Zeitpunkt der Abgabe dieser Arbeit 850 Einzelversuche gefahren.

Die Erzeugung der Anströmbedingungen in der Messstrecke geschieht durch eine Expansion des Versuchsgases durch eine Überschalldüse. Die Anlage arbeitet nach dem Stoßrohrprinzip, um die benötigten Ruhebedingungen für die Überschalldüse zu erzeugen. Der Begriff Hochenthalpie bezieht sich bei der Benennung dieser Anlage auf die maximal erreichbare Enthalpie des Gases im Düsenreservoir. Im HEG liegt die Grenze augenblicklich bei $23 \mathrm{MJ} / \mathrm{kg}$. Um diese hohen Enthalpien im Düsenreservoir und damit die benötigten hohen Absolutgeschwindigkeiten in der Messstrecke erzeugen zu können, wird die Verdichtung des Treibgases im HEG mittels eines frei fliegenden Kolbens durchgeführt. Dieser Vorschlag geht auf Stalker zurück (Stalker (1966), Stalker (1967), Stalker u. Plumb (1968)).

Die erste Umsetzung dieser Kompressionsmethode wurde in der Anlage T3 an der australischen Universität von Queensland (Stalker (1972)) realisiert. Später erfolgte der Bau der Anlage T4 an der Universität von Queensland im Jahr 1987 (Stalker u. Morgan (1988) und des T5 am California Institute of Technology im Jahr 1990 (Hornung u. a. (1991)). Der Bau weiterer Anlagen erfolgte in den Jahren $1995^{1}$ in den USA und $1997^{2}$ in Japan.
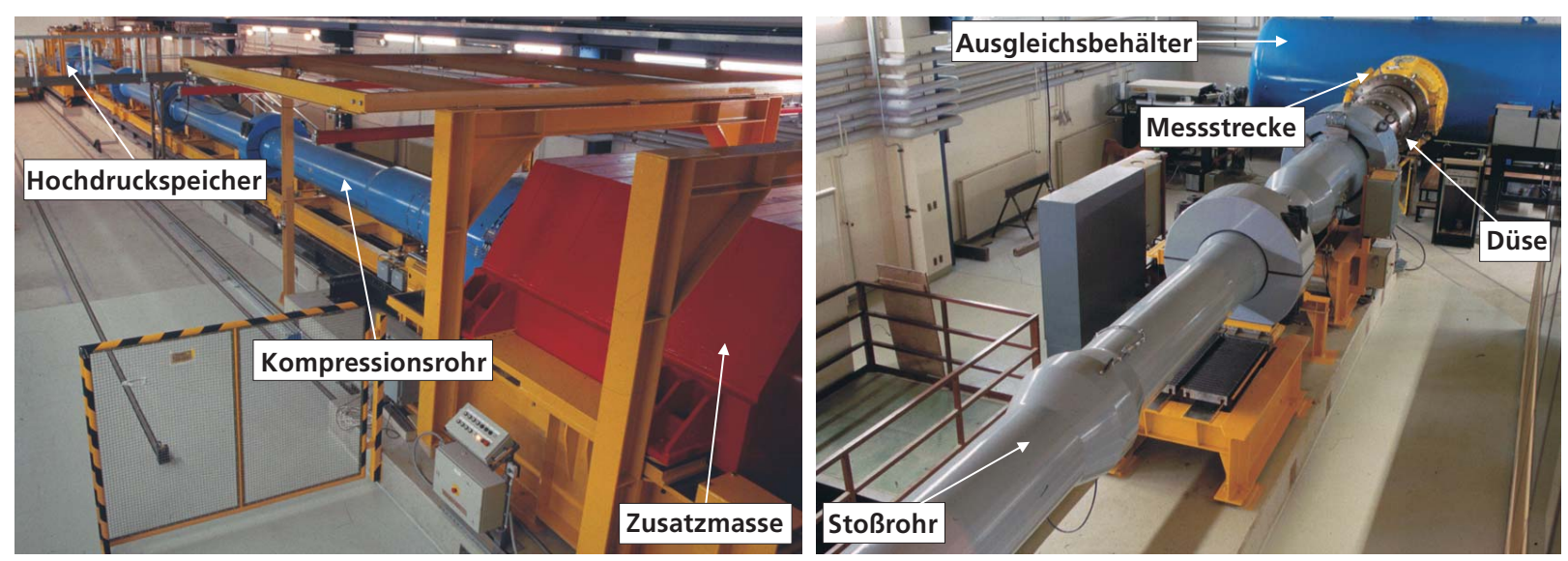

Abbildung 3.1: Photographische Ansichten des HEG.

\footnotetext{
${ }^{1} \mathrm{G} 2$, Arnold Engineering Development Center, Arnold AFB, USA

${ }^{2}$ HIEST, Japan Aerospace Exploration Agency (JAXA), Japan
} 


\subsection{Funktionsweise und Aufbau}

Die Funktionsweise des HEG basiert auf dem Stoßrohrprinzip. In Abb. $\mathbf{3 . 2}$ ist die Arbeitsweise des einfachen idealen Stoßrohres schematisch dargestellt. Das Stoßrohr wird durch eine trennende Membran in Treibrohr und Laufrohr geteilt. Nach dem Einstellen des Laufrohrzustandes (1) wird in das Treibrohr Gas eingefüllt, bis die trennende Membran bei einem Zustand (4) birst. Der Zeitpunkt $t_{1}$ stellt diese Situation kurz vor dem Bersten der Membran dar. Unter der Annahme, dass die Membran instantan verschwindet und weder molekulare noch turbulente Transportvorgänge an den Rohrwänden noch an der Mediengrenze eine Rolle spielen, würde der Druckausgleichsvorgang wie in dem Weg-Zeit-Diagramm in 3.2 dargestellt stattfinden. Im Treibgas läuft eine an der Stelle $x=0$ und $t=0$ zentrierte Expan-

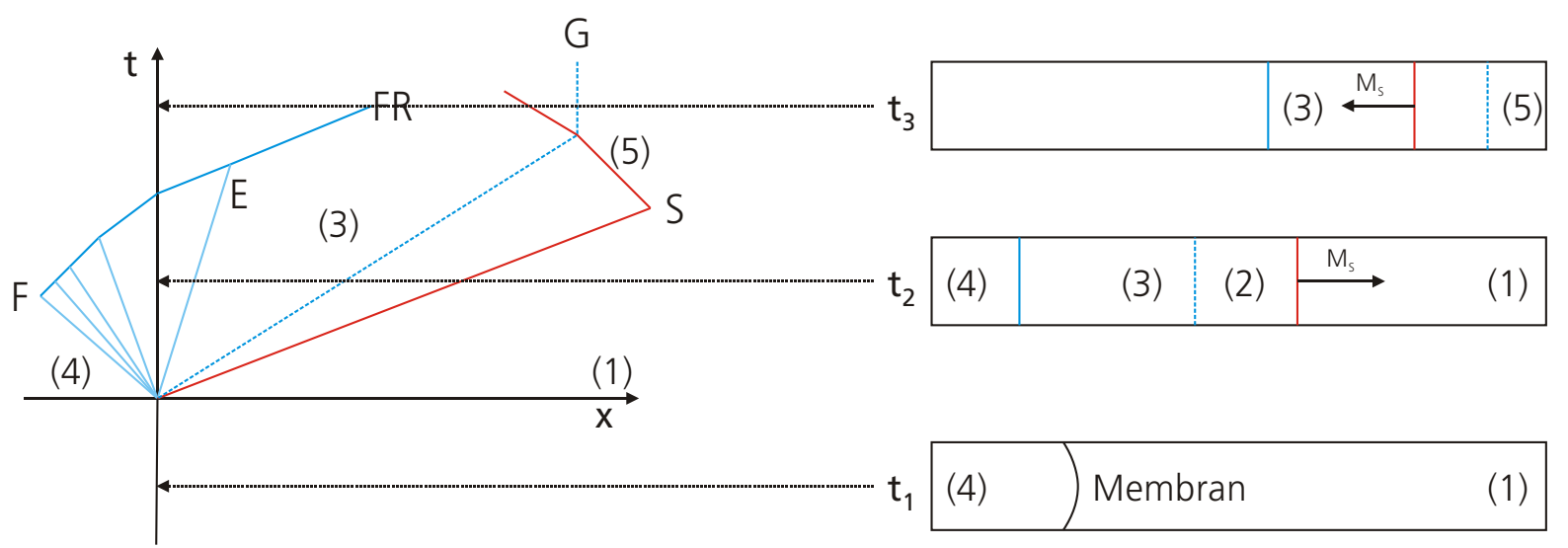

Abbildung 3.2: Wellendiagramm des einfachen Stoßrohres: (S) Verdichtungsstoß; (E) Ende der Expansionswelle; (F) Front der Expansionswelle; (FR) Front der reflektierten Expansionswelle; (G) Mediengrenze zwischen Treibgas und Laufgas.

sionswelle in Richtung Treibrohrende. Sie expandiert das Treibgas vom Zustand (4) in den Zustand (3) und beschleunigt das Treibgas auf eine Geschwindigkeit $u_{3}$ in Richtung Stoßrohrende. Die Front F dieser Expansionswelle bewegt sich mit der Schallgeschwindigkeit $a_{4}$ des vor ihr ruhenden Treibgases. Ihr Ende E bewegt sich mit der Geschwindigkeit $u_{3}-a_{3}$. E bewegt sich also im Falle $u_{3}<a_{3}$ in Richtung Treibrohrende, bleibt im Falle $u_{3}=a_{3}$ am Ort $x=0$ der Membran stehen und wird, wie in Abb. 3.2 dargestellt, im Falle $u_{3}>a_{3}$ in das Laufrohr mittransportiert. Im Laufgas wird ein Verdichtungsstoß S erzeugt, der sich mit konstanter Geschwindigkeit $u_{S}$, die größer ist als die Schallgeschwindigkeit $a_{1}$ des Laufgases, in Richtung Stoßrohrende fortpflanzt. Dieser Zustand ist zum Zeitpunkt $t_{2}$ in 3.2 dargestellt. Der Verdichtungsstoß reflektiert am Stoßrohrende und komprimiert das Testgas in den Zustand (5). Im Idealfall (angepasste Mediengrenze) bringt der Stoß die Mediengrenze (G), die dem Stoß folgt, zum Stillstand. Dies ist schematisch zum Zeitpunkt $t_{3}$ dargestellt. In der Realität sind diese Vorgänge sehr viel komplizierter, da die Membran vor dem Platzen eine Wölbung aufweist und auch mit einer endlichen Rissgeschwindigkeit öffnet. An der Wand des Laufrohres bildet sich eine Grenzschicht aus; statt einer ebenen Mediengrenze stellt sich eine turbulente Mischzone zwischen Treib- und Laufgas ein.

Die Funktionsweise des einfachen Stoßrohres lässt sich auf die Funktionsweise des HEG 
als kolbengetriebenes Stoßrohr übertragen. In Abb. 3.3 sind die Zustandsänderungen analog zum einfachen Stoßrohr für den HEG angegeben. Gegenüber dem einfachen Stoßrohr besitzt der HEG einen Kolben K für die Verdichtung des Treibgases, eine Querschnittsverengung zwischen Kompressionsrohr und Stoßrohr und eine am Stoßrohr angebrachte Überschalldüse D. Zum Zeitpunkt $t_{1}$ ist im Stoßrohr (Laufrohr beim einfachen Stoßrohr), der

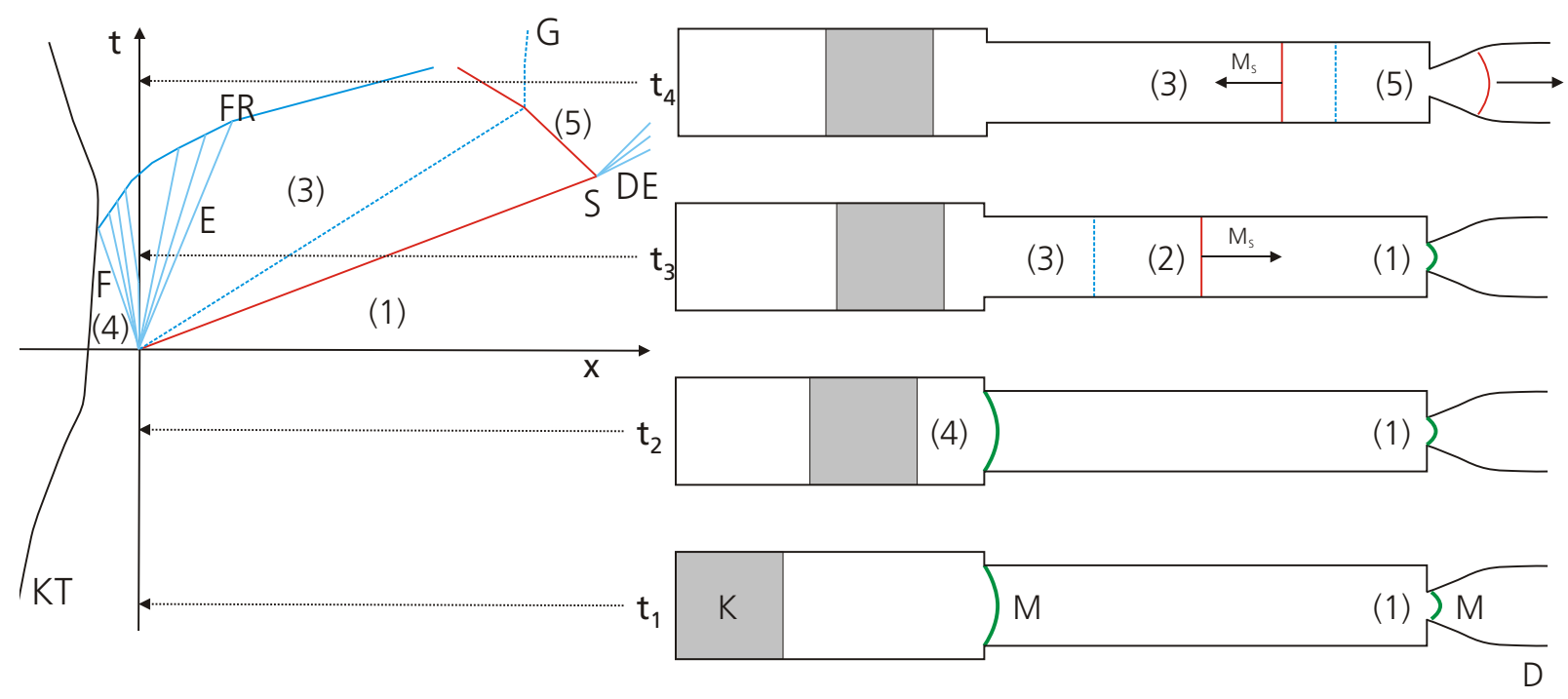

Abbildung 3.3: Wellendiagramm des HEG: (S) Verdichtungsstoß; (E) Ende der Expansionswelle; (F) Front der Expansionswelle; (FR) Front der reflektierten Expansionswelle; (G) Mediengrenze zwischen Treibgas und Laufgas; (K) Kolben; (M) Membran; (DE) Expansion durch die Überschalldüse.

Zustand (1) eingestellt. Der Kolben K wird mit Druckluft beschleunigt und komprimiert das Gas im Kompressionsrohr zwischen Kolben K und Hauptmembran M auf den Zustand (4). Der Zeitpunkt $t_{2}$ gibt den Zustand kurz vor dem Bersten der großen Hauptmembran M wieder. Das Treibrohr wird nun von der Kolbenstirnseite und der Membran gebildet. Zum Zeitpunkt $t_{3}$ ist die große Hauptmembran geborsten. Der Verdichtungsstoß bewegt sich im Stoßrohr in Richtung der Überschalldüse D. Die Kolbentrajektorie KT ist so gewählt, dass der Kolben nach dem Bersten der Hauptmembran das verbleibende Gas zwischen Kolbenstirnseite und Stoßrohr kontinuierlich weiter komprimiert, um den Massenfluss durch die Blende aufrecht zu erhalten und die Front der Expansionswelle nicht zu früh an der Kolbenstirnseite reflektieren zu lassen. Die Vorgänge im Stoßrohr laufen analog zu denen im Laufrohr beim einfachen Stoßrohr. Reflektiert der Verdichtungsstoß S am Stoßrohrende, wird durch die Zerstörung der zweiten kleinen Membran M die Düsenströmung in Gang gebracht. Im Austritt der Überschalldüse D werden Modelle platziert und vermessen. Die Duplizierung der notwendigen hohen Absolutgeschwindigkeit von $6 \mathrm{~km} / \mathrm{s}$ im Austritt der Überschalldüse D erfordert eine Enthalpie des komprimierten Laufgases von $18 \mathrm{MJ} / \mathrm{kg}$. Dies entspricht einer Stoßmachzahl $M_{S}=u_{S} / a_{1}$ des im Stoßrohr laufenden Stoßes von mindestens 12.5, wobei $a_{1}$ für Raumtemperatur gilt (Hornung (1993)). In Abb. $\mathbf{3 . 4}$ ist die Abhängigkeit des notwendigen Druckverhältnisses $p_{4} / p_{1}$ von der zu erreichenden Stoßmachzahl $M_{S}$ dargestellt. Um bei gegebenem Druckverhältnis die Stoßmachzahl zu erhöhen, muss das Verhältnis der Schallgeschwindigkeiten von Treibgas und Testgas $a_{4} / a_{1}$ möglichst groß 


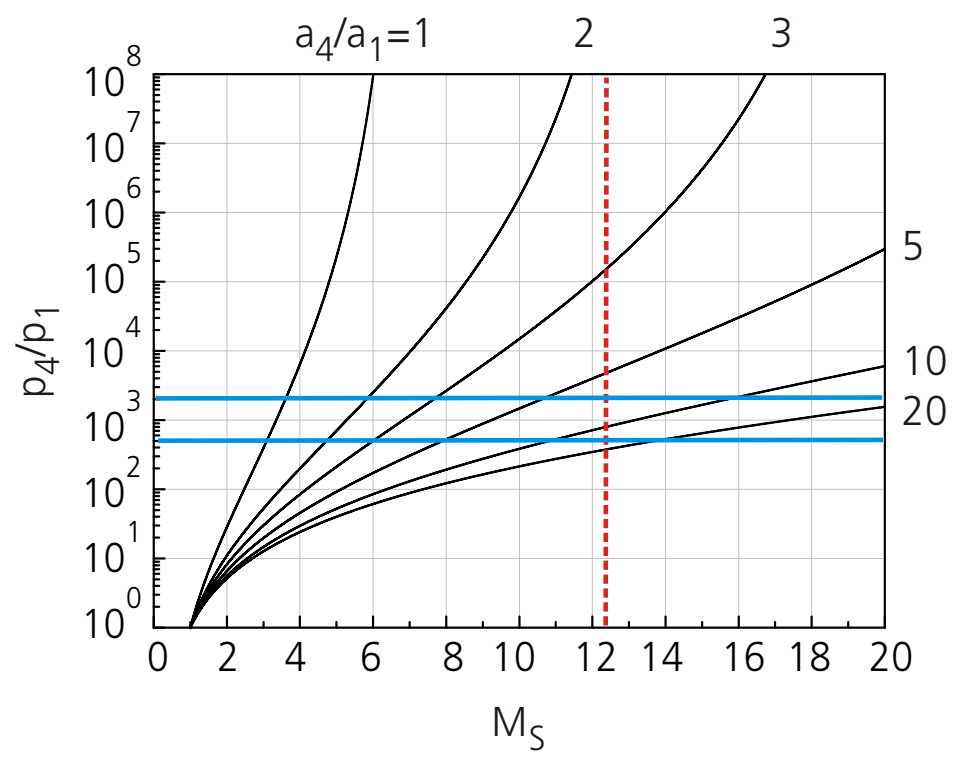

Abbildung 3.4: Abhängigkeit der Stoßmachzahl von den Füllbedingungen des Stoßrohres. Zustände analog zu den Abb. 3.2 und 3.3.

gewählt werden. Durch Kombination verschiedener Gase in Treib- und Stoßrohr lassen sich Verhältnisse bis 4 erreichen. Statisches Heizen des Treibrohres ermöglicht Verhältnisse bis ungefähr 8. Das Zünden detonationsfähiger Gasgemische im Treibrohr lässt Verhältnisse bis $12 \mathrm{zu}$. Um bei realistischen und experimentell benutzbaren Druckverhältnissen von 500 bis 2000 Stoßmachzahlen über 12 zu erhalten (blaue horizontale Linien in Abb. 3.4), kann die Kompression des Treibgases mittels eines frei fliegenden Kolbens durchgeführt werden: hier sind Verhältnisse von $a_{4} / a_{1}$ über 15 leicht realisierbar.

\begin{tabular}{|c|c|c|c|}
\hline Bezeichnung & Größe & Bezeichnung & Größe \\
\hline Gesamtlänge der Anlage & $62 \mathrm{~m}$ & Volumen des Treibluftbehälters & $5.4 \mathrm{~m}^{3}$ \\
\hline -> Gesamtgewicht & $250 \mathrm{t}$ & Länge des Überschalldüse & $3.75 \mathrm{~m}$ \\
\hline Länge des Kompressionsrohres & $33 \mathrm{~m}$ & $\rightarrow$ Austrittsdurchmesser & $0.8 \mathrm{~m}$ \\
\hline -> Durchmesser & $0.55 \mathrm{~m}$ & -> Flächenverhältnis AVA* & 1600 \\
\hline -> Volumen & $8.06 \mathrm{~m}^{3}$ & Durchmesser der Messstrecke & $1.2 \mathrm{~m}$ \\
\hline Länge des Stoßrohres & $17 \mathrm{~m}$ & -> Volumen & $2.1 \mathrm{~m}^{3}$ \\
\hline -> Durchmesser & $0.15 \mathrm{~m}$ & Volumen des Ausgleichsbehälters & $35.5 \mathrm{~m}^{3}$ \\
\hline -> Volumen & $0.301 \mathrm{~m}^{3}$ & & \\
\hline Einstellgrößen & & Bedingung I & Bedingung III \\
\hline Druck im Treibluftbehälter & & Luft bei 49.5 bar & Luft bei 49.1 bar \\
\hline Druck im Kompressionsrohr & & Helium bei 0.727 bar & He90/Ar10 bei 0.725 bar \\
\hline Druck im Stoßrohr & & Luft bei 0.24 bar & Luft bei 0.502 bar \\
\hline Dicke der Hauptmembran & & $8 \mathrm{~mm}$ & $8 \mathrm{~mm}$ \\
\hline -> Resttiefe & & $5.1 \mathrm{~mm}-5.4 \mathrm{~mm}$ & $5.1 \mathrm{~mm}-5.4 \mathrm{~mm}$ \\
\hline Dicke der Sekundärmembran & & $75 \mu \mathrm{m}$ & $75 \mu \mathrm{m}$ \\
\hline Masse des Kolbens & & $275.2 \mathrm{~kg}$ & $275.2 \mathrm{~kg}$ \\
\hline Berstdruck & & 500 bar & 500 bar \\
\hline Reservoirdruck & & 382 bar & 492 bar \\
\hline Reservoirtemperatur & & $9082 \mathrm{~K}$ & $7164 \mathrm{~K}$ \\
\hline Reservoirenthalpie & & $21.91 \mathrm{MJ} / \mathrm{kg}$ & $12.73 \mathrm{MJ} / \mathrm{kg}$ \\
\hline
\end{tabular}

Tabelle 3.1: Technische Daten des HEG. 
Der HEG lässt sich zur Funktionsbeschreibung, wie in Abb. $\mathbf{3 . 5}$ dargestellt, in folgende Abschnitte gliedern: Der Treibluftbehälter (1) dient dem Antrieb des Kolbens. Das Kompressionsrohr (2) wird an der Hauptmembranstation (3) durch eine starke Edelstahlmembran vom Stoßrohr (4) getrennt. Am Stoßrohrverschluss (5) trennt eine zweite dünne Plastikmembran die Überschalldüse (6), die das Gas in die Messstrecke (7) beschleunigt, vom Stoßrohr. Die Messstrecke, in der das Modell aufgehängt ist, ist fest mit dem Erdboden verbunden.

Der Ausgleichsbehälter (8) dient der Volumenvergrößerung der Anlage, damit der Ausgleichsdruck nach dem Versuch unter dem Atmosphärendruck bleibt. Die gesamte Anlage bis zur Düse ist im geschlossenen Zustand auf den Gleitlagern frei beweglich, um die aufgrund der Kolbenbeschleunigung entstehende Rückstoßbewegung der Anlage ausgleichen zu können. Die Düse ist über eine Gleitdichtung mit der Messstrecke verbunden. Detaillierte Angaben über Längen und Größen der Anlage sind der Tab. 3.1 zu entnehmen.

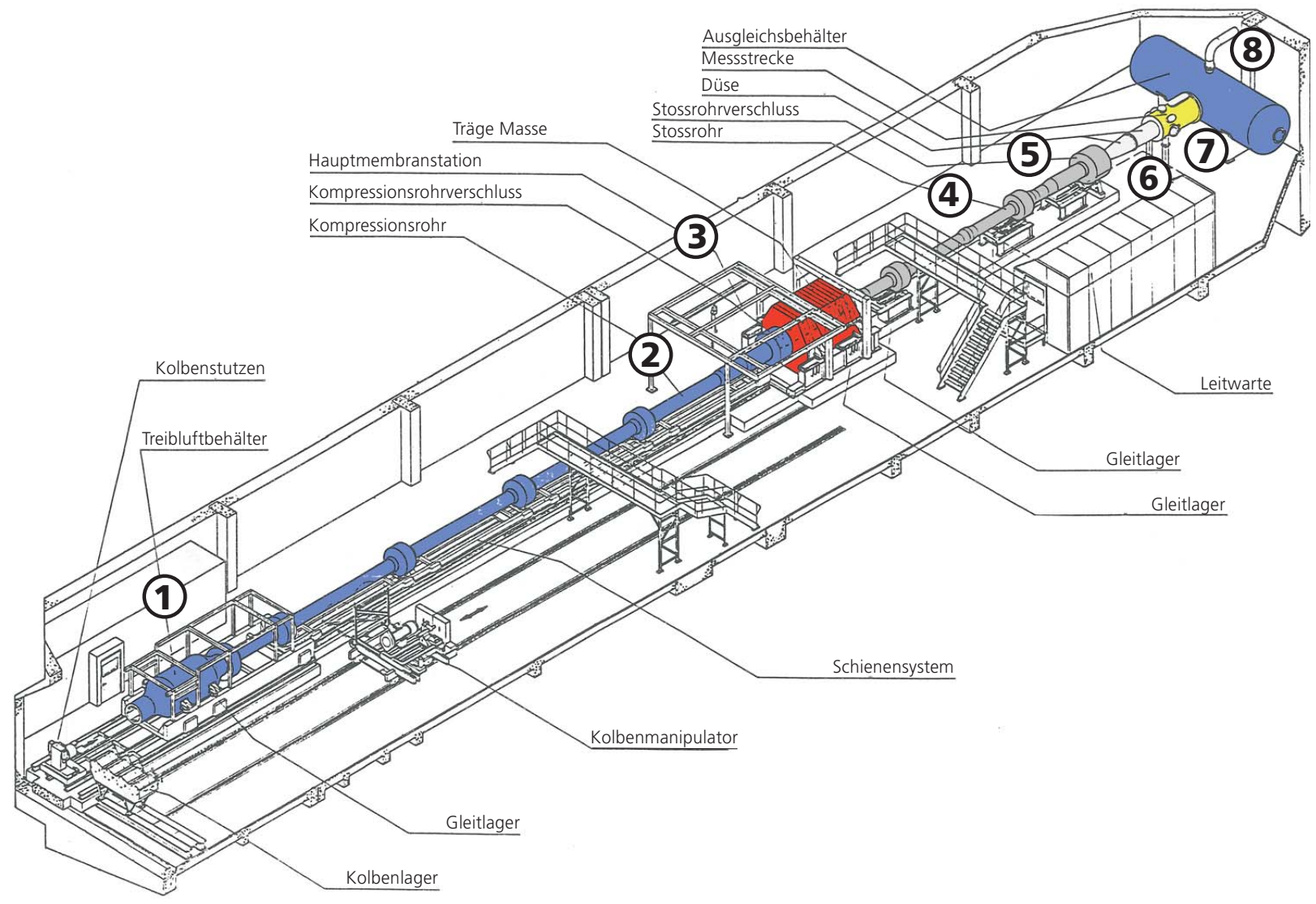

Abbildung 3.5: Darstellung des HEG: (1) Treibluftbehälter; (2) Kompressionsrohr; (3) Hauptmembranstation; (4) Stoßrohr; (5) Stoßrohrverschluss; (6) Düse; (7) Messstrecke; (8) Ausgleichsbehälter. 

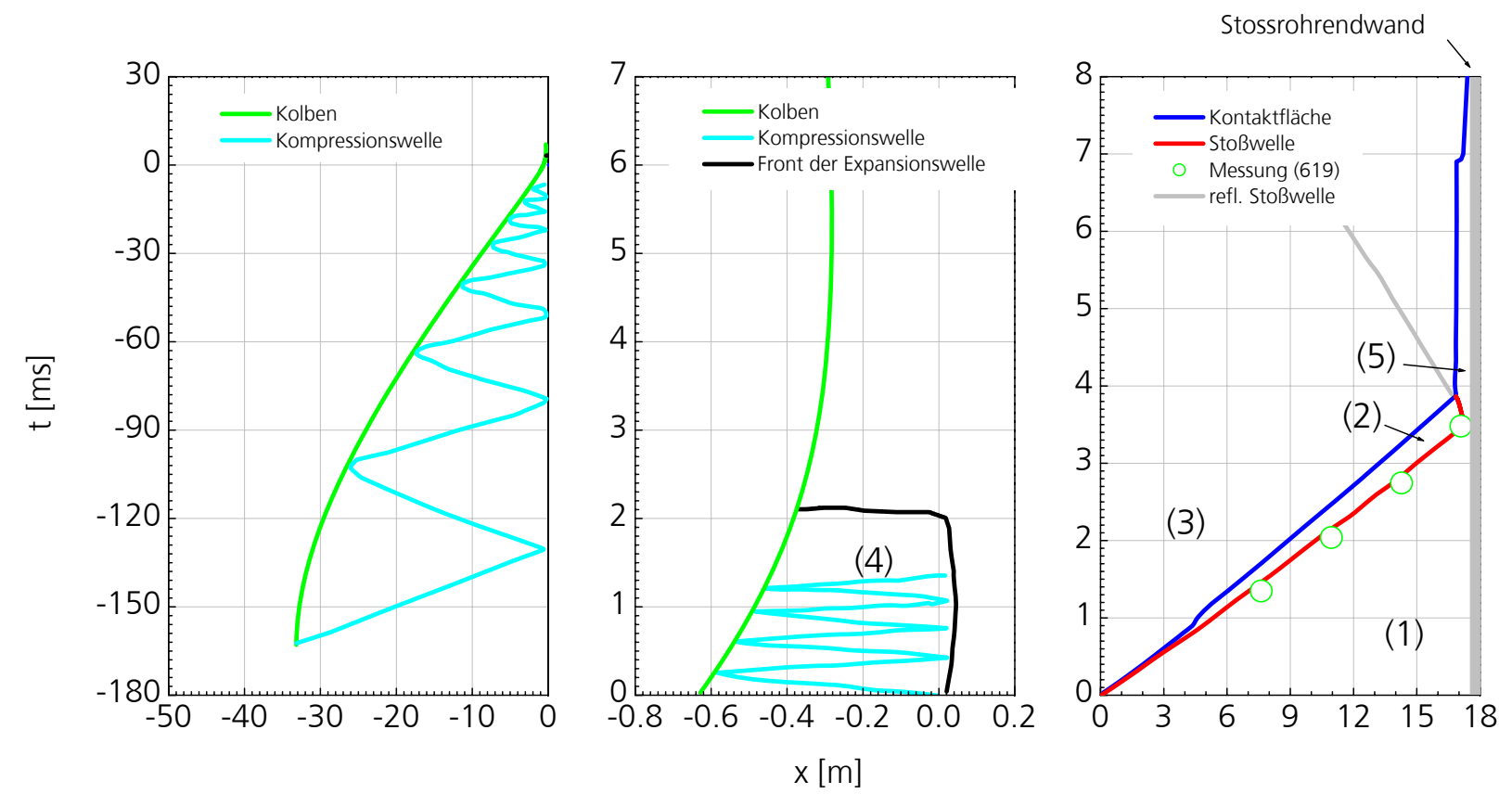

Abbildung 3.6: Numerische Simulation des HEG bei Bedingung I.

Der genaue Versuchsablauf wird im Folgenden am Beispiel der Kanalbedingung I beschrieben. In Abb. $\mathbf{3 . 6}$ ist das Weg-Zeit-Diagramm einer eindimensionalen instationären Rechnung mit dem Programm L1d (Jacobs (1999)), das auch für die Vorabauslegung neuer Betriebszustände des HEG genutzt wird (Gardner u. a. (2004)), dargestellt. Dieses Programm rechnet die Abläufe im HEG einschließlich der Kompression des Treibgases durch den Kolben. Das Weg-Zeit-Diagramm wurde in drei Teile getrennt, um die Vorgänge im Kompressionsrohr (links), an der Hauptmembran (Mitte) und im Stoßrohr (rechts) besser sichtbar zu machen, da sie auf verschiedenen Zeitskalen ablaufen. Die Simulation zeigt die HEGBedingung I. Dargestellt in der Abb. 3.6 ist die Bewegung des Kolbenmittelpunktes im Kompressionsrohr des HEG, weiterhin das Bewegungsmuster der starken Kompressionswelle, die zwischen Kolbenstirnseite und Kompressionsrohrendwand mehrfach reflektiert wird. Die Endphase der Kolbenkompression während des Berstens der Membran und die reale Ausbreitung der Front der Expansionswelle sind in der Mitte der Abb. 3.6 aufgetragen. Auf der rechten Seite sind die Ausbreitung des Verdichtungsstoßes und der Mediengrenze zwischen Treibgas und Testgas dargestellt. Die Nomenklatur der Gaszustände (1)-(5) ist analog zu Abb. 3.3. In Abb. 3.6 sind zusätzlich die Stoßgeschwindigkeitsmessungen im Stoßrohr für Versuch 619 (HEG-Bedingung I) eingetragen. Für diese Bedingung wird die Anlage mit einem Kolben von 282 kg bestückt. Die Stärke der Hauptmembran aus Edelstahl, die Kompressionsrohr und Stoßrohr trennt, beträgt $8 \mathrm{~mm}$. Der Antriebsdruck im Hochdruckspeicher beträgt 49.5 bar. Das Kompressionsrohr wird mit Helium bei einem Druck von 0.727 bar befüllt. Die Stärke der Sekundärmembran aus Mylar, die Stoßrohr und Düse trennt, beträgt $75 \mu \mathrm{m}$. Im Stoßrohr befindet sich Luft bei einem Druck von 0.24 bar. Die Überschalldüse, die Messstrecke und der Ausgleichsbehälter sind bis auf einen Druck von 0.1 mbar evakuiert. Nach dem Beaufschlagen des Kolbens mit dem Treibdruck bewegt sich der Kolben im Kompressionsrohr stromab und komprimiert das Helium innerhalb von 0.162 Sekun- 
den auf einen Druck von 500 bar. Der Kolben befindet sich dann $0.2 \mathrm{~m}$ vor dem Ende des Kompressionsrohrs; die Hauptmembran birst bei dem durch die Ritztiefe der Sollbruchstellen (5.3 mm, siehe Abschnitt 3.3.3) festgelegten Berstdruck von 390 bar. Die starken Druckänderungen, die die Kolbenstirnseite im Kompressionsgas erzeugt, steilen sich zu einer Kompressionswelle auf, die dem Kolben vorweg läuft und während der Kompression ständig zwischen Kompressionsrohrendwand und Kolbenstirnfläche reflektiert. Das in das Stoßrohr austretende Helium verdichtet das Testgas, und eine starke Stoßwelle eilt der Kontaktfläche zwischen dem Helium und der Luft voraus. Die Ankunft der Stoßwelle wird in der Stoßrohrwand an 5 Orten gemessen (s. Abschnitt 3.3.2). Die Geschwindigkeit der Stoßwelle wird über die Lauflänge des Stoßrohrer kleiner und beträgt bei dieser Bedingung als Mittelwert zwischen den beiden letzten Messstationen $4600 \mathrm{~m} / \mathrm{s}$. Erreicht die Stoßwelle die Endwand des Stoßrohres, reflektiert sie und bringt die beschleunigte Luft wieder zur Ruhe. Die komprimierte Luft bildet nun bei einem Druck von ca. 380 bar und einer Temperatur von $9000 \mathrm{~K}$ das Reservoir für die Düsenexpansion. Die Mylarmembran verdampft, und die Luft wird durch die Düse beschleunigt. Nachdem sich die Düsenströmung eingestellt hat, stellt sich auch die Umströmung des Modells ein, und die Messzeit beginnt. Nach wenigen Millisekunden trifft das Treibgas in der Messstrecke ein und beendet die Messzeit. Ist der größte Teil des Treibgases aus dem Kompressionsrohr entwichen, wird der Kolben von der verbleibenden Treibluft in Richtung Kompressionsrohrende auf einen Kupferring geschoben. Nach dem Versuch liegt noch der Hochdruck der Treibluft im Kompressionsrohr. Diese Treibluft wird abgelassen und die Messstrecke auf Atmosphäre gebracht, dann kann die Anlage wieder geöffnet werden. In den folgenden Abschnitten werden die einzelnen Bereiche der Anlage und die Messungen, die in ihnen stattfinden, genauer diskutiert. Diese Messungen dienen nicht nur zur Sicherstellung der Reproduzierbarkeit der Versuchsbedingungen, sondern auch zur Bestimmung der freien Anströmung in der Messstrecke, die das Modell umströmt. 


\subsection{Durchgeführte Versuche}

Die in dieser Arbeit durchgeführten Versuche sind als Übersicht in Tab. $\mathbf{3 . 2}$ zusammengefasst. Die Versuche sind nach den verwendeten Windkanalmodellen getrennt aufgelistet. Für die Versuche wurden die HEG-Bedingungen I und III benutzt. Diese Bedingungen unterscheiden sich bezüglich der Ruheenthalpie. Die Bedingung I hat eine nominale Ruheenthalpie von $22 \mathrm{MJ} / \mathrm{kg}$, während die Bedingung III $12 \mathrm{MJ} / \mathrm{kg}$ zur Verfügung stellt.

\begin{tabular}{|c|c|c|c|}
\hline $\begin{array}{c}\text { Bedingung I } \\
\text { Versuch }\end{array}$ & Versuchsgas & \begin{tabular}{|c|} 
Bedingung III \\
Versuch
\end{tabular} & Versuchsgas \\
\hline & Doppelrampe & & Doppelrampe \\
\hline 456 & Luft & 446 & Luft \\
\hline 457 & Luft & 447 & Luft \\
\hline 491 & Luft & 452 & Luft \\
\hline 492 & Luft & 453 & Luft \\
\hline 493 & Luft & 454 & Luft \\
\hline \multirow[t]{2}{*}{494} & Luft & 455 & Luft \\
\hline & X-38 Modell & 458 & Luft \\
\hline 551 & Luft & 459 & Luft \\
\hline 552 & Luft & 460 & Luft \\
\hline 553 & Luft & & X-38 Modell \\
\hline 554 & Luft & 517 & Luft \\
\hline 555 & Luft & 544 & Luft \\
\hline 560 & Luft & 545 & Luft \\
\hline 561 & Luft & 546 & Luft \\
\hline \multirow[t]{2}{*}{562} & Luft & 556 & Luft \\
\hline & Zylinder & 557 & Luft \\
\hline \multirow{6}{*}{$\begin{array}{l}618 \\
619 \\
620 \\
621 \\
625 \\
626\end{array}$} & Luft & 558 & Luft \\
\hline & Luft & 559 & Luft \\
\hline & Luft & & Zylinder \\
\hline & Luft & 611 & Luft \\
\hline & $\mathrm{N}_{2}$ & 615 & $\mathrm{~N}_{2}$ \\
\hline & $\mathrm{N}_{2}$ & 617 & $\mathrm{~N}_{2}$ \\
\hline \multirow[t]{3}{*}{632} & Luft & 627 & Luft \\
\hline & & 628 & Luft \\
\hline & Holographische Interferometrie & & Hochgeschwindigkeitssichtbarmachung \\
\hline
\end{tabular}

Tabelle 3.2: Übersicht der durchgeführten HEG-Experimente.

Jeder Versuch wird zeitaufgelöst untersucht. Druckmessungen und Wärmestromdichtemessungen, ob an den Windkanalmodellen oder in der Anlage, werden über einen Zeitraum von $1 \mathrm{~s}$ digital aufgenommen und gespeichert. Die Auswahl des Messzeitfensters, welches typischerweise in der Größenordnung von $0.2 \mathrm{~ms}$ bis $0.3 \mathrm{~ms}$ liegt, findet nach dem Versuch statt. Da die digitale Aufnahme von mehreren Sensoren (redundante Triggerung) ausgelöst werden kann, findet nachträglich eine zeitliche Normierung der Datensätze statt. Der Zeitpunkt $t=0$ bezieht sich immer auf den Zeitpunkt, an dem der Stoß an der Stoßrohrendwand reflektiert wird. Dieser Zeitpunkt wird im Folgenden mit SR bezeichnet. Der Zeitversatz zwischen diesem Stoßreflektionszeitpunkt und der Ankunft des Testgases in der Messstrecke (TL) und das ausgewählte Messzeitfenster sind in Tab. 3.3 für jeden Versuch angegeben. Im nachfolgenden Abschnitt werden die einzelnen Messungen an der Anlage beschrieben, die dazu dienen, den Ruhezustand des Testgases vor der Expansion zu bestimmen und damit 


\begin{tabular}{|c|c|c|c|c|c|c|c|}
\hline $\begin{array}{c}\text { Bedingung I } \\
\text { Versuch }\end{array}$ & SR [ms] & TL [ms] & Messzeit [ms] & $\begin{array}{c}\text { Bedingung III } \\
\text { Versuch }\end{array}$ & SR [ms] & $\mathrm{TL}$ [ms] & Messzeit [ms] \\
\hline 456 & 2.523 & 0.665 & $2.0-2.3$ & 446 & 3.588 & 0.787 & $2.5-2.7$ \\
\hline 457 & 2.527 & 0.714 & $2.1-2.3$ & 447 & 3.854 & 0.767 & $2.8-3.0$ \\
\hline 491 & 2.628 & 0.817 & $2.4-2.5$ & 452 & 3.670 & 0.776 & $2.6-2.8$ \\
\hline 492 & 2.639 & 0.794 & $2.5-2.7$ & 453 & 3.499 & 0.767 & $2.5-2.7$ \\
\hline 493 & 2.602 & 0.782 & $2.8-3.0$ & 454 & 3.441 & 0.782 & $2.5-2.8$ \\
\hline 494 & 2.634 & 0.779 & $2.8-3.0$ & 455 & 3.411 & 0.749 & $2.5-2.7$ \\
\hline 551 & 3.485 & 0.696 & $2.0-2.3$ & 458 & 3.400 & 0.754 & $2.5-2.6$ \\
\hline 552 & 3.429 & 0.694 & $2.0-2.3$ & 459 & 3.487 & 0.779 & $2.8-3.0$ \\
\hline 553 & 3.498 & 0.678 & $2.0-2.3$ & 460 & 3.449 & 0.758 & $2.5-2.7$ \\
\hline 554 & 3.394 & 0.703 & $2.0-2.3$ & 517 & 3.517 & 0.785 & $2.5-2.7$ \\
\hline 555 & 3.420 & 0.703 & $2.0-2.3$ & 544 & 4.619 & 0.809 & $2.8-3.0$ \\
\hline 560 & 3.414 & 0.664 & $2.1-2.3$ & 545 & 4.660 & 0.809 & $2.8-3.0$ \\
\hline 561 & 3.436 & 0.694 & $2.0-2.3$ & 546 & 4.615 & 0.814 & $2.8-3.0$ \\
\hline 562 & 3.398 & 0.686 & $2.0-2.3$ & 556 & 4.360 & 0.754 & $2.3-2.5$ \\
\hline 618 & 2.591 & 0.648 & $2.2-2.4$ & 557 & 4.557 & 0.771 & $2.2-2.4$ \\
\hline 619 & 2.603 & 0.641 & $1.9-2.1$ & 558 & 4.002 & 0.779 & $2.1-2.3$ \\
\hline 620 & 2.598 & 0.638 & $2.4-2.5$ & 559 & 3.860 & 0.775 & $2.4-2.6$ \\
\hline 621 & 2.598 & 0.637 & $2.5-2.6$ & 611 & 3.314 & 0.745 & $2.5-2.7$ \\
\hline 625 & 2.586 & 0.619 & $2.2-2.4$ & 615 & 3.334 & 0.776 & $2.6-2.8$ \\
\hline 626 & 2.695 & 0.672 & $2.2-2.4$ & 617 & 3.361 & 0.775 & $2.6-2.8$ \\
\hline \multirow[t]{2}{*}{632} & 2.671 & 0.672 & $2.0-2.3$ & 627 & 3.393 & 0.774 & $2.5-2.7$ \\
\hline & & & & 628 & 3.416 & 0.771 & $2.5-2.7$ \\
\hline \multicolumn{2}{|c|}{$\begin{array}{l}\text { Mittelwert TL [mm] } \\
\sigma_{\mathrm{TL}}[\mathrm{mm}]\end{array}$} & $\begin{array}{l}0.695 \\
0.055\end{array}$ & & \multicolumn{2}{|c|}{$\begin{array}{l}\text { Mittelwert TL [mm] } \\
\sigma_{\mathrm{TL}}[\mathrm{mm}]\end{array}$} & $\begin{array}{l}0.775 \\
0.018\end{array}$ & \\
\hline
\end{tabular}

Tabelle 3.3: Tabellierte Werte für die bestimmte Stoßreflektionszeit (SR), den Zeitversatz der Signale zwischen Düsenreservoir und Messstrecke (TL) und das definierte Messzeitfenster für die Auswertung für alle Versuche.

die freie Anströmung in der Messstrecke zu charakterisieren. In den Abschnitten 3.3.4 und 3.3.5 wird das Vorgehen im Einzelnen erläutert.

\subsection{Messungen während eines Versuches}

\subsubsection{Kanalbewegung}

Die Rückstoßbewegung des HEG wird während eines Versuches gemessen, um den Abstand des Düsenaustritts zum Modell zu bestimmen. Dieser Abstand wird bei der Bestimmung der freien Anströmung, die das Modell in der Messstrecke erfährt, berücksichtigt. Zwischen dem Kompressionsrohr und einem erdfesten Punkt ist ein induktiver Wegaufnehmer $^{3}$ eingebaut, der die Rückstoßbewegung der Anlage misst. Der Messfehler des Aufnehmers beträgt $+/-0.5 \mathrm{~mm}$. In Abb. 3.7 ist eine Messung für Bedingung I aufgetragen. Die Zeitachse ist auf den Zeitpunkt der Stoßreflektion (SR) am Stoßrohrende normiert. Die Kanalbewegung ist für den gesamten Zeitraum, den die Datenerfassung aufnimmt, in $\mathbf{A b b}$. 3.7a dargestellt und als Ausschnitt in Abb. 3.7b. Die Position $x=0 \mathrm{~mm}$ ist die Position, an der die Anlage in Richtung der Vorwärtsbewegung einen Anschlag hat.

Der Zeitraum, in dem die Versuchsauswertung typischerweise stattfindet, liegt im Bereich von 2 ms bis 3 ms nach dem Stoßreflektionszeitpunkt. In Tab. 3.5 sind die innerhalb des

\footnotetext{
${ }^{3}$ Model IW254/200, Nr. 3155, TWK-ELEKTRONIK GmbH, Heinrichstr. 85, 40239 Düsseldorf. [www.twk.de]
} 
für den Einzelversuch definierten Messzeitfensters (s. Tab. 3.3) bestimmten Mittelwerte der Kanalpositionen angegeben. Die Standardabweichungen aller Mittelwerte für Bedingung I und III sind ebenfalls in der Tab. 3.5 eingetragen.
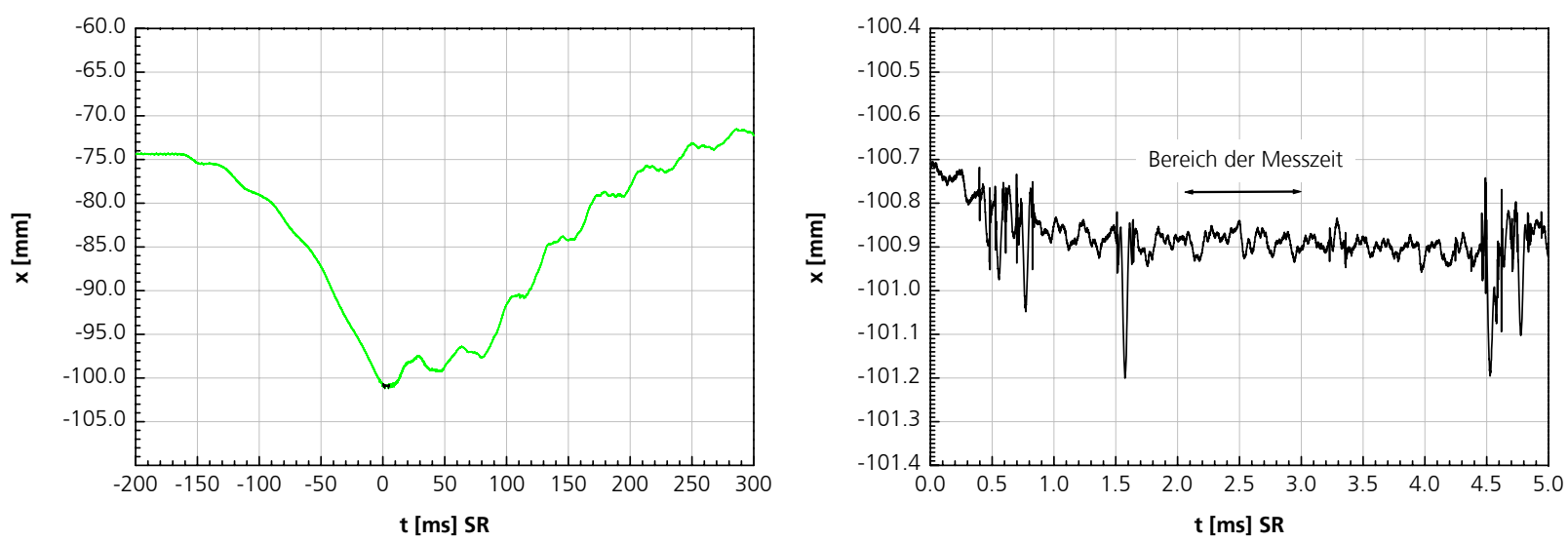

Abbildung 3.7: Auftragung der Kanalbewegung für Bedingung I. Zeitachse auf den Stoßreflektionszeitpunkt (SR) normiert. Anlagenbewegung (links) beträgt $25 \mathrm{~mm}$. In der Messzeit (rechts) ist die Anlage in Ruhe.

\subsubsection{Stoßgeschwindigkeit}

Während eines Versuchs wird die Ausbreitungsgeschwindigkeit des Verdichtungsstoßes im Stoßrohr der Anlage bestimmt. Hierfür sind 5 lonisationssensoren an verschiedenen Orten in der Stoßrohrwand eingebaut. Sie werden mit SS1 bis SS5 bezeichnet, wobei SS1 direkt an der Hauptmembranstation installiert ist und SS5 als letzter Sensor kurz vor dem Stoßrohrende. Die Signalanstiege werden elektrisch zu einem Signal zusammengefasst und aufaddiert. In Abb. $\mathbf{3 . 8}$ sind exemplarisch zwei Zeitsignale für die Versuchsbedingung I aufgetragen. Die Zeitpunkte der Anstiege für beide Versuche sind in Tab. 3.4 gelistet. Die mittlere Geschwindigkeit zwischen den Sensoren SS1 und SS2 wird unter Berücksichtigung des Abstands des Sensors SS1 zur Hauptmembran benutzt, um den Zeitpunkt des Berstens der Hauptmembran und damit den Berstdruck (CTP) zu bestimmen (s. Abschnitt 3.3.3). In Tab. 3.4 ist dieser Zeitpunkt in Rot eingetragen. Steht, wie in dem Beispiel in Abb. 3.8 für

\begin{tabular}{|ccccc|}
\hline $\begin{array}{c}\text { Auswertung Versuch 619 } \\
\text { Sensor }\end{array}$ & $\mathbf{t}$ [ms] & $\mathbf{d t}[\mathbf{m s}]$ & $\mathbf{d x}[\mathbf{m m}]$ & $\mathbf{s s} \mathbf{[ m} / \mathbf{s}]$ \\
\hline CTP & -3.658 & & & \\
SS1 & - & - & 480 & - \\
SS2 & -2.633 & - & 3950 & - \\
SS3 & -2.126 & 0.507 & 2595 & 5118.34 \\
SS4 & -1.436 & 0.690 & 3325 & 4818.84 \\
SS5 & -0.729 & 0.707 & 3325 & 4702.97 \\
STP & 0.000 & 0.729 & 3301 & 4528.12 \\
\hline
\end{tabular}

\begin{tabular}{|c|c|c|c|c|}
\hline \\
\hline \multicolumn{5}{|c|}{$\begin{array}{l}\text { Auswertung Versuch } 551 \\
\begin{array}{lll}\text { Sensor } & t \text { [ms] } & d t[m s]\end{array}\end{array}$} \\
\hline \multicolumn{5}{|c|}{$\begin{array}{ll}\text { CTP } & -3.592\end{array}$} \\
\hline \multicolumn{5}{|c|}{ SS1 $\quad-3.495$} \\
\hline & -2.699 & 0.796 & 3950 & 4962.31 \\
\hline SS3 & -2.176 & 0.523 & 2595 & 4961.76 \\
\hline \multirow{2}{*}{$\begin{array}{l}\text { SS4 } \\
\text { SS5 }\end{array}$} & -1.468 & 0.708 & 3325 & 4696.33 \\
\hline & -0.742 & 0.726 & 3325 & 4579.89 \\
\hline STP & 0.000 & 0.742 & 3301 & 4448.79 \\
\hline
\end{tabular}

Tabelle 3.4: Auswertung der mittleren Geschwindigkeiten zwischen den Messpunkten. Nullpunkt: Anstieg des Drucksensors am Ende des Stoßrohres (STP). In Rot eingetragen die ermittelten Zeiten für das Bersten der Membran (CTP). Exemplarische Auswertung der Versuches 551 und 619. 

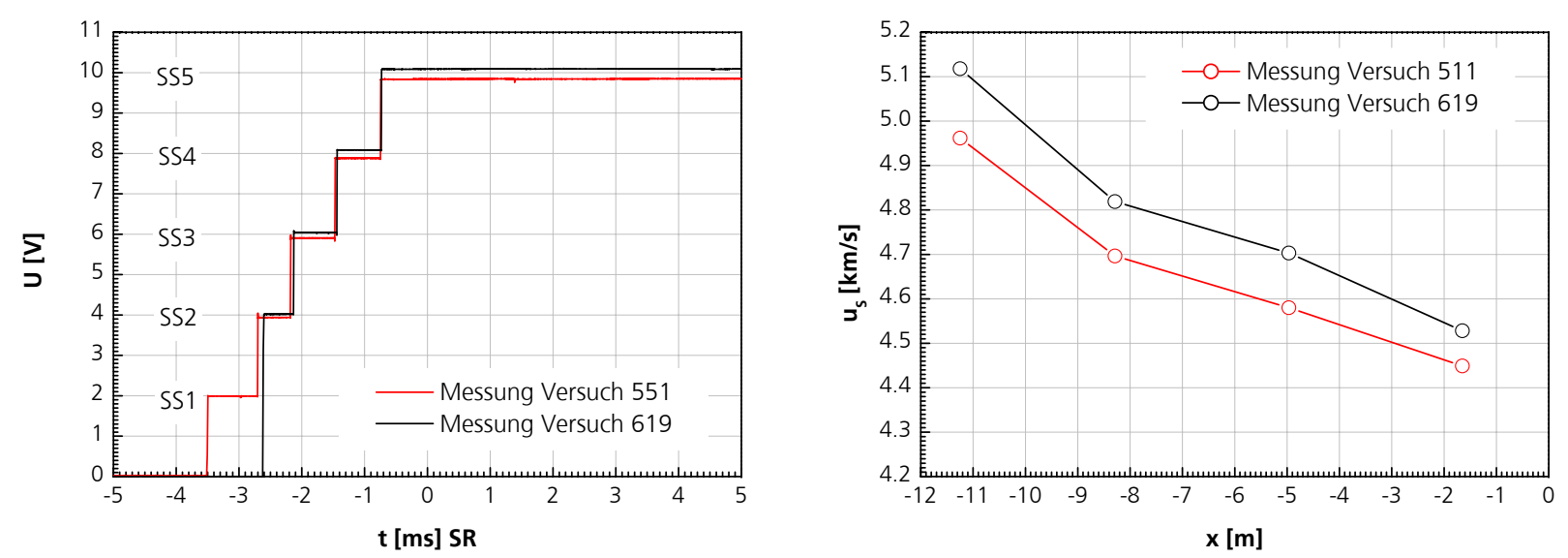

Abbildung 3.8: Beispiel für die Messung der Stoßgeschwindigkeit im Stoßrohr für die Versuche 619 und 551. Voltpegel der Sensoren: SS1 $=2 \mathrm{~V}$, SS2 $=4 \mathrm{~V}$, SS3 $=6 \mathrm{~V}$, SS4 $=8 \mathrm{~V}$ und SS5 $=10 \mathrm{~V}$. Die Anstiege der lonisationssensoren werden elektrisch zu einem Signal addiert (links). Zeitachse auf den Stoßreflektionszeitpunkt (SR) normiert. Auftragung der mittleren Geschwindigkeiten zwischen den Messstellen (rechts).

den Versuch 619, durch einen Defekt des Sensors (z.B. Abbrand der Elektrode) keine Messung von SS1 zur Verfügung, wird über die Momentangeschwindigkeit des Stoßes zwischen den Sensoren SS2 und SS3 zurück gerechnet. Dieser so bestimmte Zeitpunkt des Berstens der Membran findet sich ebenfalls rot eingetragen in Tab. 3.4. Es wird deutlich, dass die unterschiedliche Bestimmung des Berstzeitpunktes nicht zu großen Abweichungen führt. Durch die Wechselwirkung des Stoßes mit der Stoßrohrwand fällt die Stoßgeschwindigkeit mit der Lauflänge ab (s. Abb. 3.8). Zur Bestimmung des Gaszustandes im Düsenreservoir (s. Abschnitt 3.3.4) wird eine Stoßgeschwindigkeit benötigt, die die Kompression des Gases im Düsenreservoir bestimmt. Die hier benutzte Verfahrensweise geht auf Stalker (Stalker u. Morgan (1988)) zurück. Als Annahme gilt, dass die Stoßgeschwindigkeit nach 2/3 Lauflänge die Kompression des Gases definiert, das sich zur Messzeit um das Modell herum befindet. Als führende Geschwindigkeit wird der Mittelwert der Geschwindigkeiten zwischen SS3/SS4 und SS4/SS5 benutzt. In Tab. 3.5 sind die nach dem obigen Verfahren ermittelten Stoßgeschwindigkeiten für alle Versuche angegeben. Bei den Versuchen, die keinen Wert enthalten, ist aufgrund defekter Sensorik keine Auswertung möglich gewesen.

\subsubsection{Hauptmembranstation}

Der Aufbau der Hauptmembranstation, die Kompressionsrohr und Stoßrohr trennt, ist in Abb. 3.9 dargestellt. Die Hauptmembran besteht aus einer runden Platte gewalzten Edelstahls, die durch eine kreuzförmige Ausfräsung eine Sollbruchstelle besitzt. Die Resttiefe dieser Ausfräsung bestimmt den Berstdruck. Um den zeitlichen Verlauf des Berstdruckes zu messen, sind an der Hauptmembranstation 2 Druckaufnehmer eingebaut. Beide Aufnehmer messen an der Endwand des Kompressionsrohres den entstehenden Druck bei der Kompression des Treibgases. Bei den Druckaufnehmern handelt es sich um piezoelektrische 

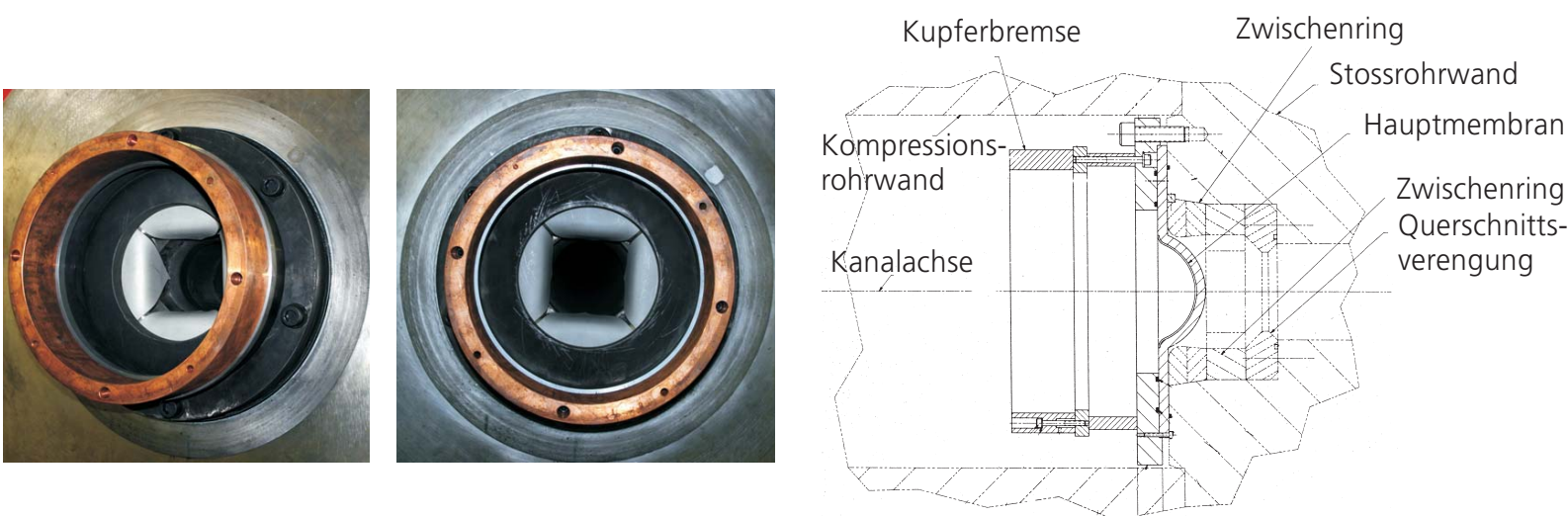

Abbildung 3.9: Aufbau der Hauptmembranstation. Die Membran ist im belasteten Zustand kurz vor den Bersten gezeichnet. Der Kupferring dient als Bremse für den Kolben.

Druckaufnehmer ${ }^{4}$, die jeweils einen eigenen Ladungsverstärker (Typ 5090, Kistler Instrumente $\mathrm{GmbH}$ ) besitzen. Diese Druckaufnehmer haben einen nominellen Messbereich von 6000 bar. Ein typisches Zeitsignal dieser beiden Aufnehmer (CTP\#1, CTP\#2) ist in Abb. 3.10 aufgetragen. Die Sensoren arbeiten nach dem piezoelektrischen Prinzip; es werden Ladungen weniger Pico-Coulomb verstärkt. Die Verstärker befinden sich außerhalb des Kompressionsrohres, deshalb müssen Kabellängen von mehreren Metern in Kauf genommen werden; hier können Ladungsverluste auftreten. Zusätzlich beträgt die Gastemperatur des komprimierten Treibgases weit über $3000{ }^{\circ} \mathrm{C}$, die Sensoren sind nur für einen Bereich bis $200{ }^{\circ} \mathrm{C}$ kompensiert. Wie stark sich die Sensoren in der kurzen Messzeit erhitzen, ist unbekannt. Zur Vermessung dieser extremen Gaszustände sind keine Sensoren käuflich erhältlich, weshalb mit den vorhandenen Sensoren gearbeitet werden muss. Eine Fehlereinschätzung für die Messung des Berstdruckes ist schwierig. Die Bestimmung des Berstzeitpunktes der Membran erfolgt mit Hilfe der Stoßgeschwindigkeitsmessung (s. Abschnitt 3.3.2). In Abb. 3.10 ist ein Ausschnitt aus der Berstdruckmessung dargestellt, in dem der für diesen Versuch ermittelte Berstzeitpunkt und die Anstiegszeiten der einzelnen lonisationssensoren in der Stoßrohrwand mit eingetragen sind. Dieses Drucksignal wird in einem Zeitfenster von +/- 0.05 ms um den Berstzeitpunkt ausgewertet. In Tab. 3.5 sind die ermittelten Berstdrücke zusammen mit den ermittelten Stoßgeschwindigkeiten für alle Versuche tabelliert.

${ }^{4}$ Typ 6215, Kistler Instrumente GmbH, Postfach 12 62, 73748 Ostfildern [www.kistler.com] 


\begin{tabular}{|c|c|c|c|c|c|c|c|}
\hline \begin{tabular}{|c|} 
Bedingung I \\
Versuch \\
\end{tabular} & $\mathrm{SS}[\mathrm{m} / \mathrm{s}]$ & CTP [bar] & $x[\mathrm{~mm}]$ & \begin{tabular}{|c|} 
Bedingung III \\
Versuch
\end{tabular} & $\mathrm{SS}$ [m/s] & CTP[bar] & $x[\mathrm{~mm}]$ \\
\hline 456 & 4894 & 506.0 & -97.1 & 446 & 3659 & 473.1 & -98.2 \\
\hline 457 & 4888 & 508.4 & -98.9 & 447 & 3571 & 493.9 & -90.6 \\
\hline 491 & 4619 & 507.1 & - & 448 & 3532 & 495.9 & -91.2 \\
\hline 492 & 4629 & 509.9 & - & 451 & 3532 & 510.7 & -88.4 \\
\hline 493 & 4706 & 505.6 & - & 452 & 3563 & 468.2 & -98.6 \\
\hline 494 & 4642 & 513.6 & - & 453 & 3554 & 451.0 & -99.4 \\
\hline 551 & 4638 & 512.3 & -96.3 & 454 & 3599 & 457.7 & -98.7 \\
\hline 552 & 4714 & 546.0 & -95.3 & 455 & 3647 & 448.7 & -91.9 \\
\hline 553 & 4652 & - & - & 458 & 3644 & 459.1 & -98.8 \\
\hline 554 & 4755 & 557.2 & -96.1 & 459 & 3576 & 449.1 & -99.9 \\
\hline 555 & 4709 & 551.4 & -95.6 & 460 & 3582 & 451.0 & - \\
\hline 560 & 4746 & 552.2 & -96.5 & 517 & 3535 & 455.0 & - \\
\hline 561 & 4685 & 554.4 & -96.3 & 544 & - & 548.2 & -98.7 \\
\hline 562 & 4744 & 538.3 & -95.9 & 545 & - & 530.1 & -98.7 \\
\hline 618 & 4782 & 503.9 & -99.9 & 546 & 3472 & 506.3 & -98.6 \\
\hline 619 & 4779 & 507.7 & -100.9 & 556 & 3744 & 581.2 & -95.7 \\
\hline 620 & 4622 & 514.0 & -100.6 & 557 & 3537 & 560.8 & -96.6 \\
\hline 621 & 4708 & 520.6 & -100.7 & 558 & 3416 & 487.6 & -96.5 \\
\hline 625 & 4713 & 508.3 & -99.3 & 559 & 3588 & 480.4 & -96.9 \\
\hline 626 & 4688 & 511.8 & -99.1 & 611 & 3737 & 444.8 & -99.0 \\
\hline \multirow[t]{4}{*}{632} & 4621 & 527.3 & -100.1 & 615 & 3708 & 451.0 & -99.5 \\
\hline & & & & 617 & 3682 & 441.3 & -99.5 \\
\hline & & & & 627 & 3642 & 447.7 & -99.6 \\
\hline & & & & 628 & - & 446.8 & -99.4 \\
\hline Mittelwert & 4711 & 522.8 & -98.03 & Mittelwert & 3596 & 480.8 & -97.01 \\
\hline$\sigma$ & 78.84 & 19.26 & 2.06 & $\sigma$ & 83.68 & 40.19 & 3.37 \\
\hline$\sigma[\%]$ & $1.67 \%$ & $3.68 \%$ & $-2.10 \%$ & $\sigma[\%]$ & $2.33 \%$ & $8.36 \%$ & $-3.47 \%$ \\
\hline
\end{tabular}

Tabelle 3.5: Tabellierte Werte der ermittelten Stoßgeschwindigkeiten (SS), Berstdrücke (CTP) und Kanalpositionen $(x)$ für alle Versuche.
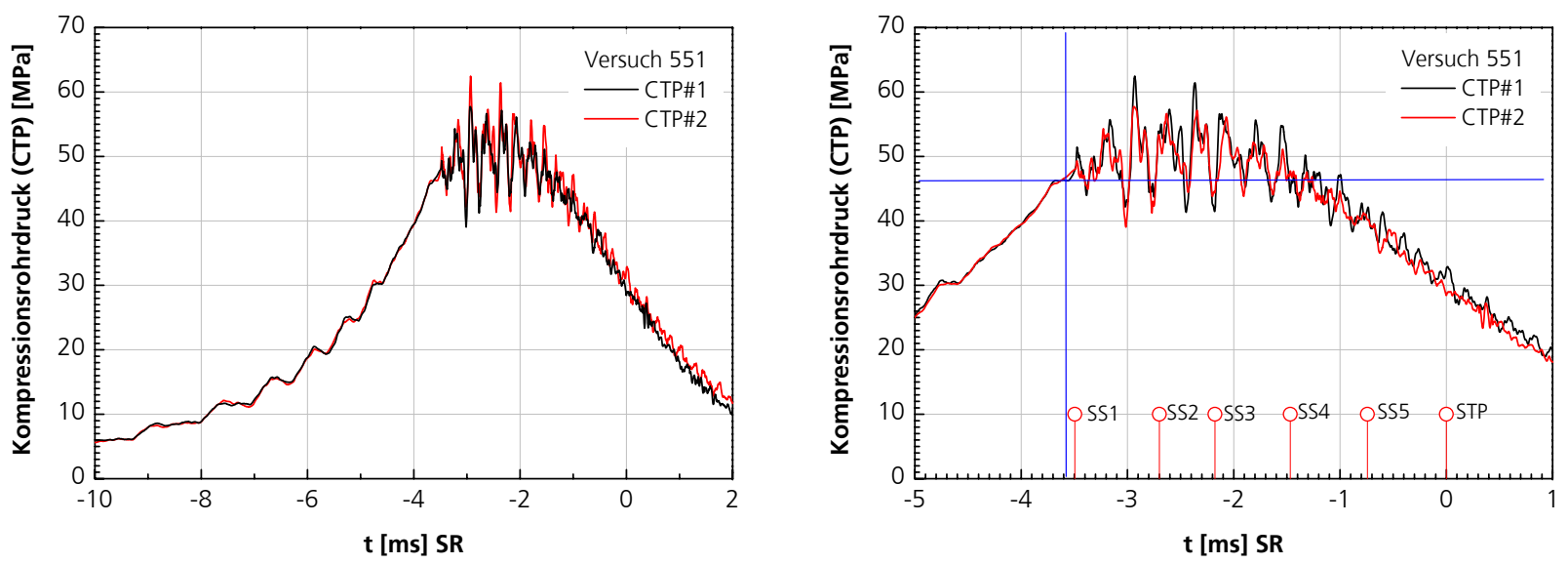

Abbildung 3.10: Auftragung des zeitlichen Verlaufes des Druckes am Ende des Kompressionsrohrs (CTP). Zeitachse auf den Stoßreflektionszeitpunkt (SR) normiert. Auftragung über einen großen Zeitabschnitt (links). Ausschnitt (rechts). Mit eingetragen ist der aus der Stoßgeschwindigkeitsmessung ermittelte Zeitpunkt des Membranberstens bei $t=-3.592 \mathrm{~ms}$. 


\subsubsection{Düsenreservoir}

Um den Zustand des Gases am Ende des Stoßrohres hinter dem reflektierten Stoß (s. Abb. 3.3, Zustand (5)) zu bestimmen, wird der zeitliche Druckverlauf von zwei in der Endwand des Stoßrohres eingebauten Drucksensoren gemessen. Diese Drucksensoren sind baugleich zu den an der Hauptmembran verwendeten (s. Abschnitt 3.3.3). In Abb. 3.11 findet sich eine Zeichnung des Stoßrohrendes mit sich anschließendem Düsenhals. Der Partikelstopper, der vor dem Düsenhals verschraubt ist, fängt kleine Metallfragmente auf, die beim Bersten der großen Hauptmembran entstehen, und hindert diese daran, durch die Düse auf das Modell zu treffen. Die Sekundärmembran aus Mylar ist in Abb. 3.11 im Unterschallbereich des Düsenhalses eingesetzt. In Abb. $\mathbf{3 . 1 2}$ sind die beiden sich ergebenden Druckverläufe bei Bedingung I, Versuch 619, dargestellt. Die ausgewerteten Ruhedrücke in den jeweili-
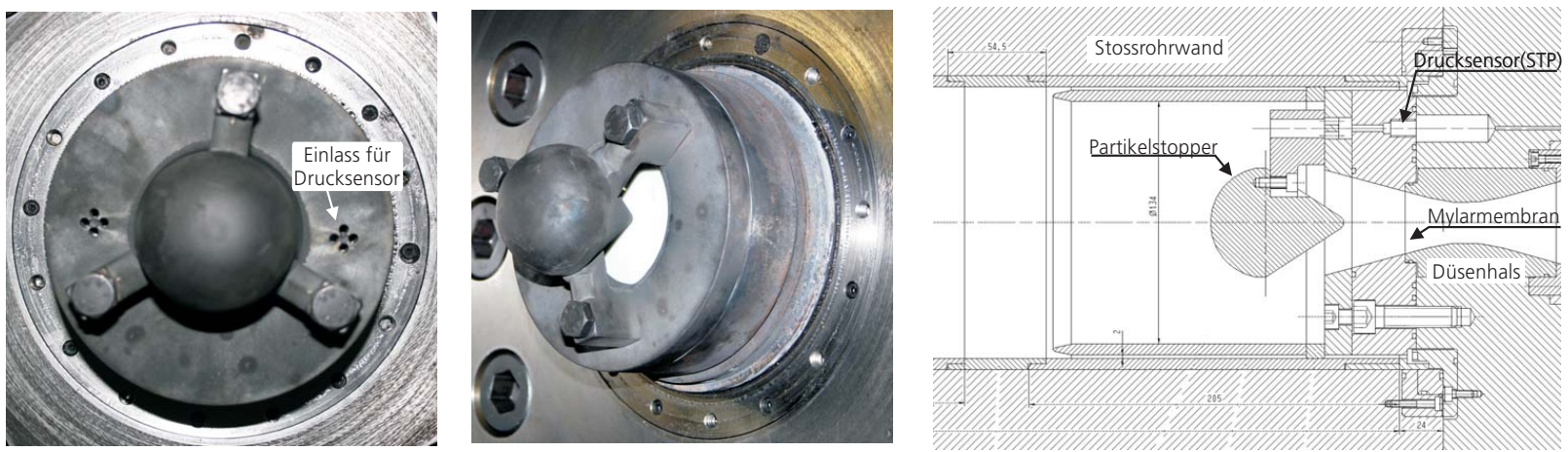

Abbildung 3.11: Aufbau der Stoßrohrendwand. Die Sekundärmembran (Mylar), die das Stoßrohr von der evakuierten Düse und Messstrecke trennt, ist hier im Unterschallbereich des Düsenhalses eingesetzt.

gen Messzeitfenstern sind in der Tab. $\mathbf{3 . 6}$ angegeben. Der Ruhedruck des Gases vor der Düsenexpansion ist somit bestimmt. Um die Temperatur des Gases stromab der reflektierten Stoßwelle (Zustand (5), Abb. 3.3) zu bestimmen, wird eine Gleichgewichtsrechnung mit dem Programm STN (Krek u. Jacobs (1993)) durchgeführt. Diese Rechnung bestimmt aus dem gemessenen Stoßrohrfülldruck und der Temperatur des Gases im Stoßrohr (Zustand (1), Abb. 3.3), dem gemessenen Berstdruck (Zustand (4), Abb. 3.3) und der gemessenen Laufgeschwindigkeit des Stoßes im Stoßrohr (s. Tab. 3.5) den Gaszustand hinter dem reflektierten Stoß. Der so errechnete Druck ist zu hoch, da das Programm die Wechselwirkung des reflektierten Stoßes mit der Grenzschicht an der Stoßrohrwand nicht berücksichtigt (Chue u. a. (1998)). Das Programm gleicht den bestimmten Gaszustand isentrop auf den gemessenen Ruhedruck an. Hiermit ist der Ruhezustand des Gases vor dem Eintritt in die Düse bestimmt. Die berechneten Werte sind in der Tab. 3.6 angegeben. 

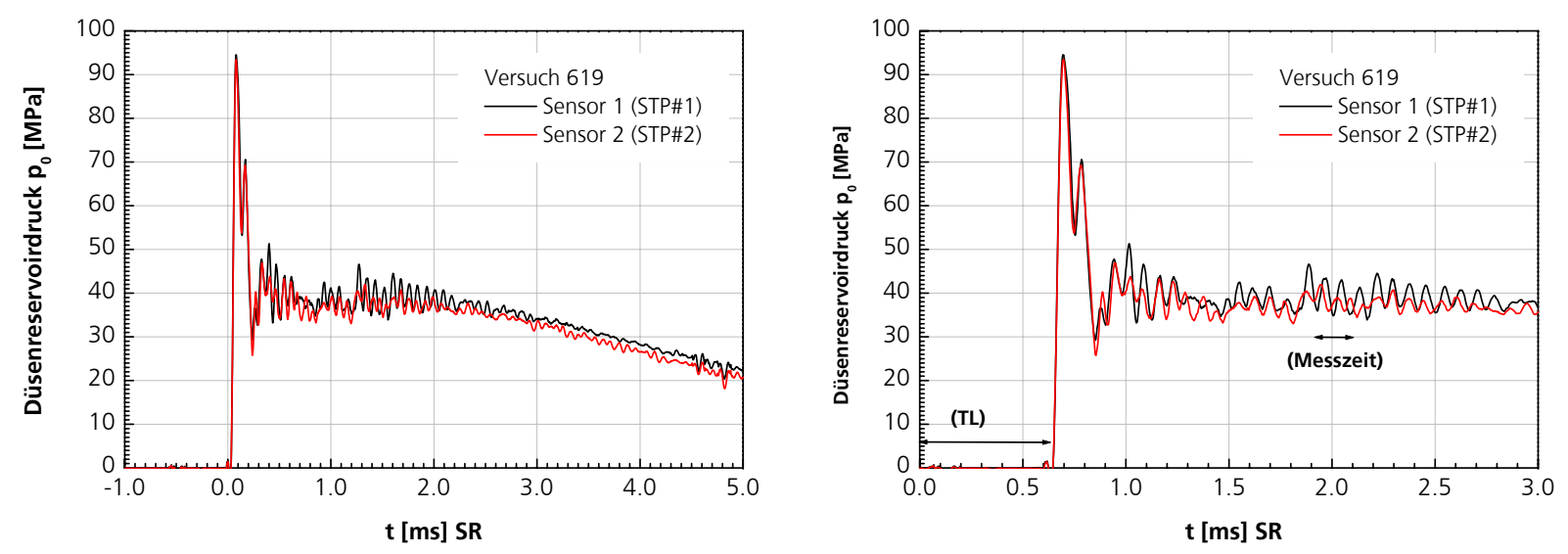

Abbildung 3.12: Auftragung der Zeitsignale der Reservoirdrucksensoren (STP\#1/STP\#2). Zeitsignal bezogen auf den Stoßreflektionszeitpunkt (links). Zeitsignal verschoben zur Auswertung des Reservoirdrucks bezogen auf die Angaben für das Messzeitfenster in Tab. 3.3 (rechts).

\begin{tabular}{|c|c|c|c|c|c|c|c|c|c|}
\hline \begin{tabular}{|c|} 
Bedingung I \\
Versuch
\end{tabular} & $\mathrm{p}_{0}[\mathrm{MPa}]$ & $\mathrm{T}_{0}[\mathrm{~K}]$ & $\rho_{0}\left[\mathrm{~kg} / \mathrm{m}^{3}\right]$ & $\mathrm{h}_{0}[\mathrm{MJ} / \mathrm{kg}]$ & \begin{tabular}{|c|}
$\begin{array}{c}\text { Bedingung III } \\
\text { Versuch }\end{array}$ \\
\end{tabular} & $\mathrm{p}_{0}[\mathrm{MPa}]$ & $\mathrm{T}_{0}[\mathrm{~K}]$ & $\rho_{0}\left[\mathrm{~kg} / \mathrm{m}^{3}\right]$ & $\mathrm{h}_{0}[\mathrm{MJ} / \mathrm{kg}]$ \\
\hline 456 & 36.7 & 9309 & 9.71 & 23.57 & 446 & 46.7 & 7251 & 19.22 & 13.01 \\
\hline 457 & 36.5 & 9294 & 9.66 & 23.49 & 447 & 44.3 & 7017 & 19.03 & 12.39 \\
\hline 491 & 30.3 & 8755 & 8.80 & 20.64 & 452 & 44.9 & 7016 & 19.29 & 12.38 \\
\hline 492 & 28.5 & 8702 & 8.44 & 20.48 & 453 & 45.5 & 7013 & 19.58 & 12.36 \\
\hline 493 & 31.6 & 8925 & 8.92 & 21.45 & 454 & 42.1 & 7022 & 18.05 & 12.44 \\
\hline 494 & 33.2 & 8898 & 9.36 & 21.10 & 455 & 45.4 & 7192 & 18.89 & 12.86 \\
\hline 551 & 44.7 & 9224 & 12.25 & 22.23 & 458 & 43.0 & 7131 & 18.06 & 12.73 \\
\hline 552 & 44.2 & 9288 & 11.92 & 22.71 & 459 & 41.5 & 6959 & 17.95 & 12.28 \\
\hline 553 & 43.4 & 9196 & 11.93 & 22.16 & 460 & 43.8 & 7030 & 18.75 & 12.44 \\
\hline 554 & - & 9176 & 10.45 & 22.48 & 517 & 46.3 & 6975 & 20.08 & 12.24 \\
\hline 555 & 43.8 & 9272 & 11.85 & 22.63 & 544 & 47.7 & 7135 & 20.08 & 12.66 \\
\hline 560 & 44.4 & 9331 & 11.86 & 22.99 & 545 & 48.3 & 7148 & 20.28 & 12.68 \\
\hline 561 & 44.8 & 9270 & 12.16 & 22.53 & 546 & 48.9 & 6911 & 21.47 & 12.04 \\
\hline 562 & 44.1 & 9377 & 11.66 & 23.33 & 556 & 52.8 & 7516 & 20.79 & 13.70 \\
\hline 618 & 40.5 & 9276 & 10.87 & 22.96 & 557 & 54.5 & 7149 & 22.97 & 12.59 \\
\hline 619 & 39.4 & 9233 & 10.65 & 22.76 & 558 & 52.3 & 6857 & 23.23 & 11.89 \\
\hline 620 & 38.3 & 9100 & 10.65 & 21.96 & 559 & 51.9 & 7062 & 22.19 & 12.39 \\
\hline 621 & 39.2 & 9116 & 10.88 & 21.98 & 611 & 52.0 & 7487 & 20.58 & 13.62 \\
\hline 625 & 39.1 & 9161 & 10.75 & 22.29 & 615 & 50.6 & 7425 & 20.24 & 13.46 \\
\hline 626 & 39.8 & 9149 & 10.98 & 22.15 & 617 & 49.1 & 7343 & 19.90 & 13.24 \\
\hline \multirow[t]{2}{*}{632} & 40.3 & 9068 & 11.35 & 21.56 & 627 & 48.7 & 7239 & 20.12 & 12.94 \\
\hline & & & & & 628 & 48.8 & 7164 & 20.47 & 12.72 \\
\hline Mittelwert & 39.1 & 9149 & 10.72 & 22.26 & Mittelwert & 47.7 & 7138 & 20.06 & 12.68 \\
\hline$\sigma$ & 5.0 & 186 & 1.17 & 0.85 & $\sigma$ & 3.7 & 180 & 1.45 & 0.48 \\
\hline$\sigma[\%]$ & $12.86 \%$ & $2.03 \%$ & $10.93 \%$ & $3.84 \%$ & $\sigma[\%]$ & $7.71 \%$ & $2.52 \%$ & $7.24 \%$ & $3.82 \%$ \\
\hline
\end{tabular}

Tabelle 3.6: Tabellierte Werte für den Ruhezustand des Gases vor der Düsenexpansion für alle Versuche. 


\subsubsection{Messungen in der Messstrecke}

In der Messstrecke der Anlage ist eine permanente Sonde installiert, die während jedes Versuchs den zeitlichen Verlauf des Pitot-Drucks ${ }^{5} p_{t 2}$, des statischen Drucks $p_{\infty}$ und der Wärmestromdichte auf einer Halbkugel $\left(D=20 \mathrm{~mm}, 0^{\circ}, 45^{\circ}, 90^{\circ}\right)$ misst. In Abb. $\mathbf{3 . 1 3}$ sind diese Messungen für den Versuch 619 dargestellt. In dem für den Versuch 619 gewähl-
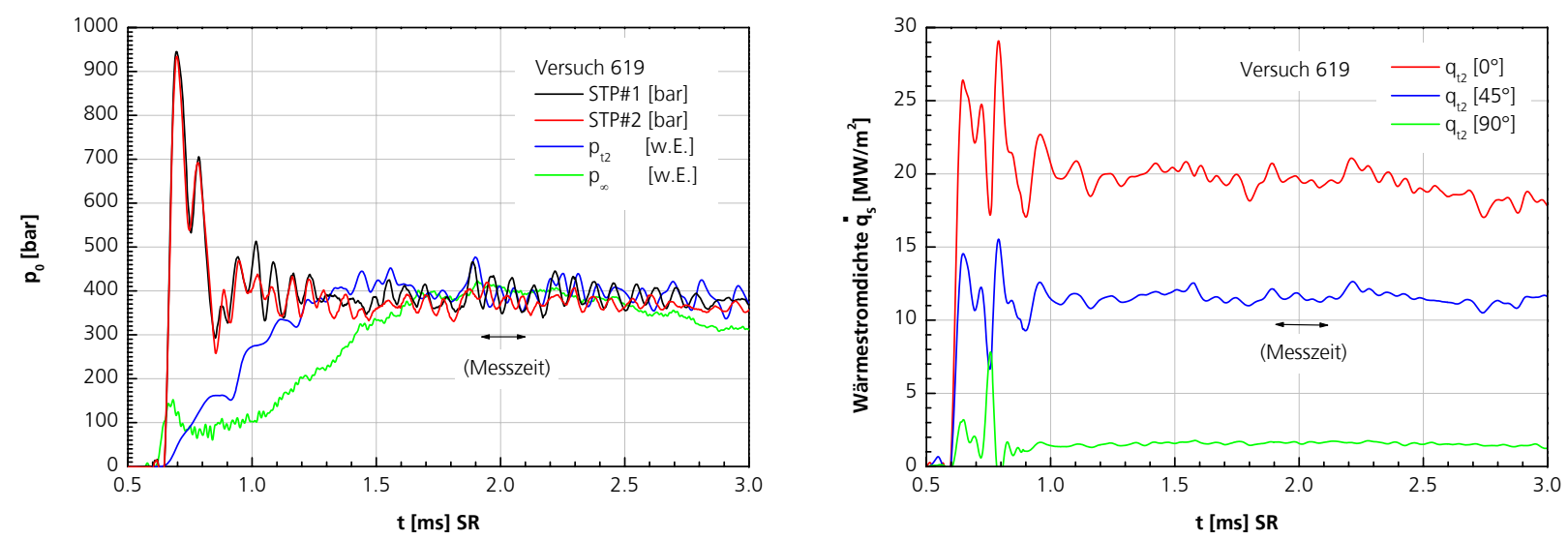

Abbildung 3.13: Auftragung der Zeitsignale der permanenten Sonde für Versuch 619. Die Zeitachse ist bezogen auf den Zeitpunkt der Stoßreflektion (SR). Auf der linken Seite der Reservoirdruck im Vergleich mit dem Pitot-Druck $p_{t 2}$ und dem statischen Druck $p_{\infty}$. Der Pitot-Druck und der statische Druck sind skaliert in willkürlichen Einheiten dargestellt, um einen Vergleich mit den Ruhedrucksignale zu ermöglichen. Der zeitliche Verlauf der Wärmestromdichte auf der Halbkugel unter den Winkeln $0^{\circ}, 45^{\circ}$ und $90^{\circ}$, bezogen auf die Anströmung, ist im rechten Bild dargestellt.

ten Messzeitfenster ist in Abb. 3.13 zu erkennen, dass die Werte für den Pitot-Druck und den statischen Druck konstante ${ }^{6}$ Werte annehmen. Das Verhältnis vom Reservoirdruck zum Pitot-Druck und zum statischen Druck ist ebenfalls konstant. Dies ist ein wichtiges Kriterium für die Wahl des Messzeitfensters. In Abb. 3.13 ist auch zu erkennen, dass der statische Druck bei 2.5 - 2.6 ms nach Stoßreflektion abzufallen beginnt, während der Pitot-Druck noch ein konstantes Verhalten aufweist. Es muss eine Zustandsänderung im Gas ablaufen, die im statischen Druck sichtbar wird, im Reservoirdruck und im Pitot-Druck jedoch nicht. Für das Verhältnis vom gemessenen Staudruck $p_{t 2}$ zum statischen Druck der freien Anströmung $p_{\infty}$ gilt:

$$
\frac{p_{t 2}}{p_{\infty}}=\left(\frac{\kappa+1}{2} M_{1}^{2}\right)^{\frac{\kappa}{\kappa-1}}\left(\frac{\kappa+1}{2 \kappa M_{1}^{2}-(\kappa-1)} M_{1}^{2}\right)^{\frac{\kappa}{\kappa-1}} .
$$

Mit den Annahmen $\kappa=7 / 5$ und $M_{1}^{2}>7 \Longrightarrow M_{1}^{2}=M_{1}^{2}-1$ lässt sich obige Gleichung zu

$$
\frac{p_{t 2}}{p_{\infty}} \approx 1.287 M_{1}^{2}
$$

reduzieren. Diese Annahmen setzen voraus, dass der Nasenradius der Pitot-Sonde so klein ist, dass das reagierende Gas seine Zusammensetzung zwischen Stoß und Körper nicht än-

\footnotetext{
${ }^{5}$ Dies ist der gemessene Staudruck $p_{t 2}$. Der Index 2 beziffert die Messung hinter einem Stoß. Das Staudruckrohr oder Pitot-Rohr (engl: pitot tube) wurde nach Henri de Pitot (1695-1771) benannt.

${ }^{6}$ Konstanz nach Maßstäben für Impulsanlagen wie den HEG
} 
dert, also die Strömung hier als eingefroren zu betrachten ist. In Abb. $\mathbf{3 . 1 4}$ ist der zeitliche Verlauf des Verhältnisses vom gemessenen Pitot-Druck zum statischen Druck unter Verwendung der Formel 3.2 aufgetragen. Es ist deutlich erkennbar, dass ab 2.5 ms nach Stoßreflektionszeitpunkt die Machzahl zu steigen beginnt, wie es bei der Ankunft des Treibgases Helium zu erwarten wäre. Die Ankunftszeit limitiert das Messzeitfenster. Eine Auswertung dieses Versuches zu einem späteren Zeitpunkt ist nicht möglich.

Die Messung der Wärmestromdichte auf der Halbkugel kann als Energiemonitor betrachtet werden. Die empirische Korrelation von Verant (1995) verknüpft $p_{t 2}$ und $h_{0}$, um die Staupunktwärmestromdichte auf einer Kugel mit beliebigen Radius zu erhalten:

$$
q_{t 2}=\sqrt{\frac{p_{t 2}}{R}} \cdot 23.787 \cdot\left(\frac{h_{0}-h_{\text {wall }}}{R_{g} \cdot T_{\text {ref }}}\right)^{1.0688},
$$

wobei $R_{g}=287.015 \mathrm{~J} /(\mathrm{kg} * \mathrm{~K})$ und $T_{\text {ref }}=273.15 \mathrm{~K}$ Konstanten sind und die Wandenthalpie $h_{\text {wall }}=c_{p} T_{\text {wall }}$ bei Raumtemperatur bestimmt wird. In Tab. 3.7 sind die Messungen der permanenten Sonde für alle Versuche gegeben. Für jeden Einzelversuch ist unter Verwendung von Formel 3.3 in ihrer umgestellten Form:

$$
h_{0}=R_{g} \cdot T_{r e f}\left(\frac{q_{t 2}}{23.787} \cdot \sqrt{\frac{R}{p_{t 2}}}\right)^{\frac{1}{1.0688}}+h_{\text {wall }}
$$

die Ruheenthalpie bestimmt worden. Die Abweichungen der Mittelwerte zwischen diesen Verfahren und der Bestimmung mit STN (s. Abschnitt 3.3.4) betragen -16\% für die Bedingung I und $-10.8 \%$ für die Bedingung III. Dass die Messung des statischen Druckes in einer
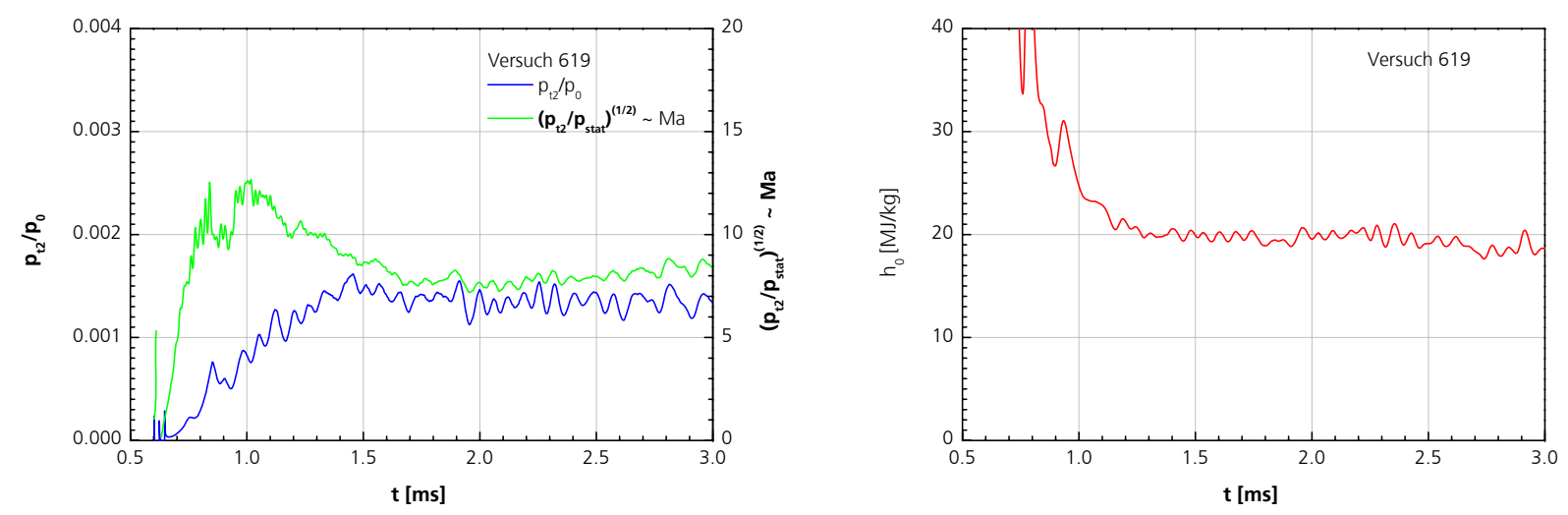

Abbildung 3.14: Auftragung der normierten Zeitsignale der permanenten Sonde für Versuch 619. Die Zeitachse ist bezogen auf den Zeitpunkt der Stoßreflektion (SR).

Impulsanlage wie dem HEG problematisch ist, soll an der Entwicklung der statischen Sonde aufgezeigt werden. Für diese Messungen wird eine Lanzensonde verwendet. Erste Messungen im HEG mit einer an der RWTH Aachen entwickelten Sonde (Kindl u. Olivier (1995), Kindl u. Olivier (1996)) wurden von Schnieder (1999) publiziert. Die Rohsignale dieser Sonde wiesen starke Schwingungen auf, und eine Auswertung war nur mittels starker Glättung möglich. Um die Signalqualität zu verbessern, wurde die Sondenform vom Autor geändert. 


\begin{tabular}{|c|c|c|c|c|c|c|c|c|c|}
\hline \multicolumn{5}{|l|}{ Bedingung I } & \multicolumn{5}{|l|}{ Bedingung III } \\
\hline Versuch & $\mathrm{p}_{\mathrm{t} 2}[\mathrm{kPa}]$ & $\mathbf{p}_{\infty}[\mathrm{Pa}]$ & $\mathrm{q}_{\mathrm{t} 2}\left[\mathrm{MW} / \mathrm{m}^{2}\right]$ & $\mathrm{h}_{0}[\mathrm{MJ} / \mathrm{kg}]$ & Versuch & $\mathrm{p}_{\mathrm{t} 2}[\mathrm{kPa}]$ & $\mathbf{p}_{\infty}[\mathrm{Pa}]$ & $\mathrm{q}_{\mathrm{t} 2}\left[\mathrm{MW} / \mathrm{m}^{2}\right]$ & $\mathrm{h}_{0}[\mathrm{MJ} / \mathrm{kg}]$ \\
\hline 456 & 52.79 & - & 18.43 & 17.86 & 446 & 66.59 & - & 11.75 & 10.38 \\
\hline 457 & 52.22 & - & 18.88 & 18.37 & 447 & 64.65 & - & 11.13 & 10.00 \\
\hline 491 & 48.14 & - & 18.62 & 18.85 & 452 & 67.28 & - & 12.75 & 11.18 \\
\hline 492 & 47.45 & - & 17.19 & 17.59 & 453 & 57.21 & - & 10.74 & 10.25 \\
\hline 493 & 48.91 & - & 16.97 & 17.12 & 454 & 54.28 & - & 10.94 & 10.70 \\
\hline 494 & 51.37 & - & 17.28 & 17.02 & 455 & 60.53 & - & 10.95 & 10.16 \\
\hline 551 & 55.72 & 758 & 23.27 & 21.73 & 458 & 60.40 & - & 11.50 & 10.66 \\
\hline 552 & 51.99 & 748 & 21.80 & 21.11 & 459 & 58.03 & - & 10.23 & 9.71 \\
\hline 553 & 51.63 & 827 & 21.17 & 20.59 & 460 & 62.13 & - & 10.13 & 9.31 \\
\hline 554 & 52.86 & 813 & 22.31 & 21.41 & 517 & 71.88 & 661 & 13.90 & 11.77 \\
\hline 555 & 55.10 & 866 & 22.37 & 21.04 & 544 & 66.89 & 606 & 13.57 & 11.90 \\
\hline 560 & 57.72 & 954 & 23.26 & 21.36 & 545 & 59.19 & 652 & 12.80 & 11.93 \\
\hline 561 & 58.49 & 916 & 22.93 & 20.94 & 546 & 67.21 & 673 & 13.20 & 11.56 \\
\hline 562 & 57.61 & 967 & 23.19 & 21.32 & 556 & 70.19 & 823 & 15.50 & 13.22 \\
\hline 618 & 50.47 & 731 & 19.19 & 18.96 & 557 & 74.63 & 778 & 14.65 & 12.16 \\
\hline 619 & 51.12 & 696 & 19.98 & 19.58 & 558 & 74.42 & 719 & 13.70 & 11.42 \\
\hline 620 & 54.10 & 680 & 20.33 & 19.39 & 559 & 73.52 & 786 & 14.73 & 12.31 \\
\hline 621 & 53.79 & 677 & 19.91 & 19.05 & 611 & 68.00 & 657 & 13.05 & 11.37 \\
\hline 625 & 54.06 & 487 & 20.70 & 19.72 & 615 & 64.85 & 667 & 13.31 & 11.86 \\
\hline 626 & 62.10 & 669 & 20.31 & 18.14 & 617 & 63.74 & 690 & 13.12 & 11.80 \\
\hline \multirow[t]{2}{*}{632} & 70.50 & 714 & 24.71 & 20.57 & 627 & 70.50 & 710 & 13.78 & 11.78 \\
\hline & & & & & 628 & 70.11 & 717 & 13.97 & 11.97 \\
\hline Mittelwert & 54.20 & 767 & 20.61 & 19.61 & Mittelwert & 65.74 & 701 & 12.70 & 11.25 \\
\hline$\sigma$ & 5.17 & 127 & 2.25 & 1.49 & $\sigma$ & 5.80 & 67 & 1.55 & 0.95 \\
\hline$\sigma[\%]$ & $9.54 \%$ & $16.55 \%$ & $10.93 \%$ & $7.61 \%$ & $\sigma[\%]$ & $8.83 \%$ & $9.61 \%$ & $12.17 \%$ & $8.45 \%$ \\
\hline
\end{tabular}

Tabelle 3.7: Messungen auf der permanenten Sonde.

Die Schwingungen im Drucksignal der Sonde, die auf dem Entwurf der RWTH Aachen basiert (i.F. Sonde 1), sind in Abb. $\mathbf{3 . 1 5}$ dargestellt. Zum Vergleich sind auch die Rohsignale der Messungen des statischen Druckes mit der modifizierten Sonde (i.F. Sonde 2) aufgetragen. Die Verbesserung in der Rohsignalqualität ist deutlich ersichtlich. Diese Verbesserung basiert auf einer Schwingungsisolation des Sensors in der Lanzensonde. Diese erste Modifikation bestand in einer Vergrößerung des Durchmessers der Sonde von $4 \mathrm{~mm}$ auf $5 \mathrm{~mm}$, um mehr Platz für eine Schwingungsisolation des Sensors zu schaffen. Um bessere Justagemöglichkeiten für die Sonde zu schaffen, wurde im nächsten Schritt das Anschlußstück an den Sondenhalter geändert. In Abb. $\mathbf{3 . 1 6}$ sind die Änderungen von Sonde 2 zur endgültigen Form (Sonde 3) zu sehen. Die Abstände von der Sondenspitze zu den Druckanbohrungen und von den Druckanbohrungen zum Schaft sind nicht verändert worden. Der Winkel, unter dem der Sondenschaft auf seinen Endradius läuft, wurde von $10^{\circ}$ auf $15^{\circ}$ vergrößert, um die Gesamtlänge der Sonde zu verkleinern. Abb. 3.17 enthält die technische Zeichnung der endgültigen Form. Die Messungen des statischen Druckes bei den Versuchen für die X-38-Konfiguration (Abschnitt 5.2) wurden ebenfalls mit der Sonde 2 durchgeführt. Bei den Messungen am Zylinder (Abschnitt 5.1) wurde die Sonde 3 benutzt. Aufgrund der Abmessungen der Sonde muss ein Drucksensor benutzt werden, der einen Messbereich von 35 kPa hat (KULITE XCS 093). Die statischen Drücke in der Messstrecke bei den benutzten HEG-Bedingungen (Bed. III u. I) liegen bei ca. 700 Pa. Dieser Druck liegt bei $2 \%$ des angebenen Messbereiches des Sensors. Neuere Untersuchungen (Sonntag (2005)) haben gezeigt, dass die Annahme einer linearen Kennkurve in diesem Bereich mit Fehlern behaftet ist. Eine spezielle Niederdruckkalibrierung, die für diese Sensoren durchgeführt wurde, 

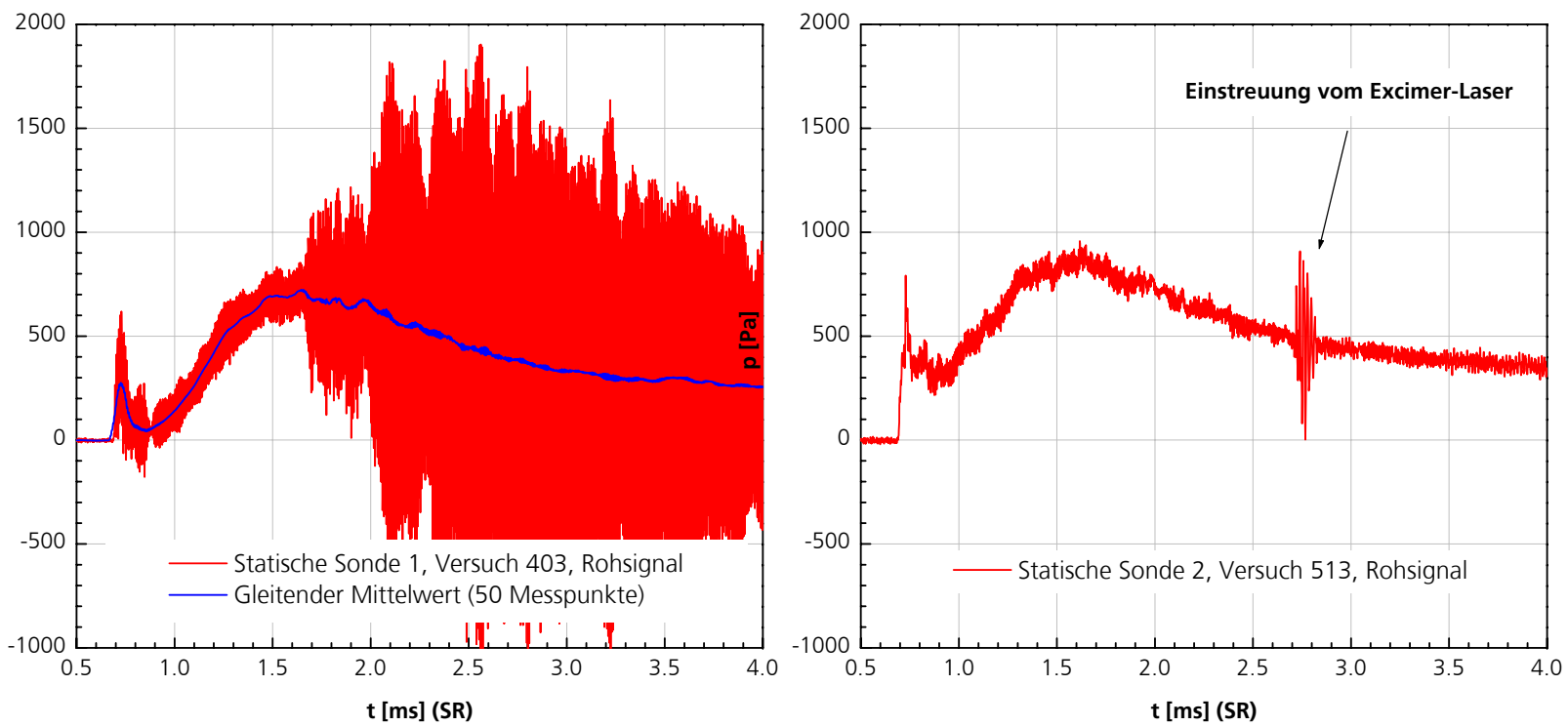

Abbildung 3.15: Vergleich der Rohsignale der Lanzensonden 1 und 2.

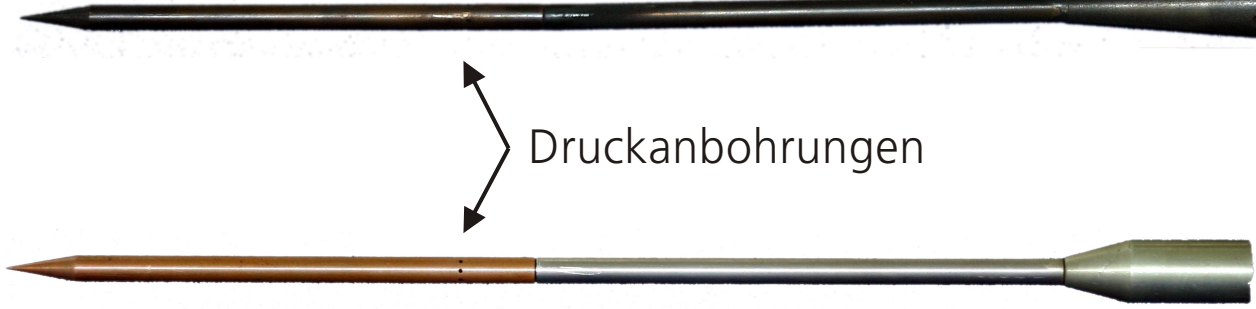

Abbildung 3.16: Vergleich Sonde 2 und 3.

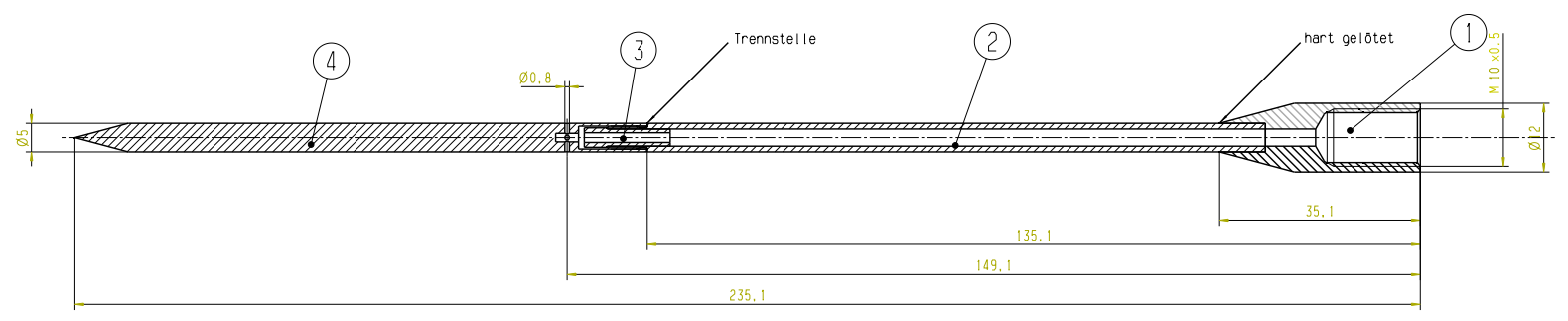

Abbildung 3.17: Lanzensonde für die Messung des statischen Druckes: (1) Sondenschaft mit Schraubgewinde zum Anschluß an die Sondenhalterung (Neusilber); (2) Grundkörper (Neusilber); (3) Sensorhalter (Edelstahl); (4) Vorkörper aus Kupfer.

ist in Abb. 3.18 zusammen mit einer linearen Kennkurve dargestellt. Die Annahme einer linearen Kennkurve führt auf zu hohe Drücke. Die Korrekturkurve in Abb. 3.18 ergibt eine Drucküberhöhung von $12 \%$ bei 700 Pa für diesen speziellen Sensor. Ein zusätzlicher Fehler ensteht durch den verbleibenden Restdruck oder Hintergrunddruck in der Messstrecke des HEG vor Versuchsbeginn. Der Hintergrunddruck im HEG wird momentan auf Vorschlag des 

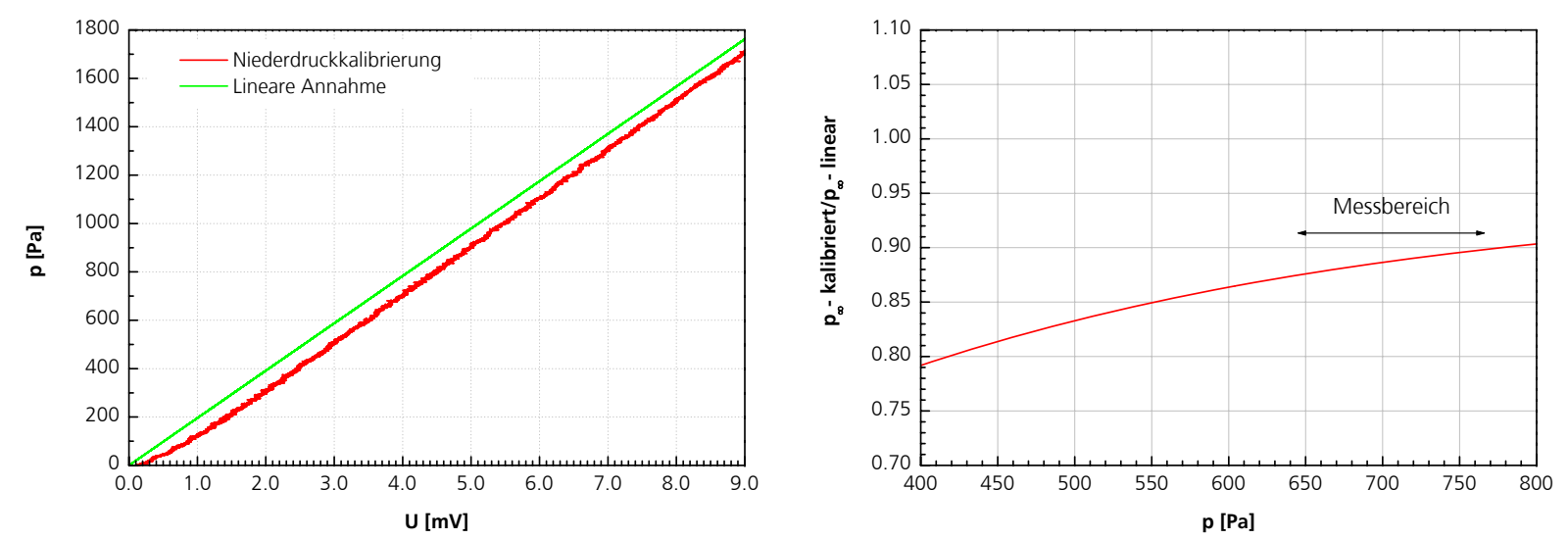

Abbildung 3.18: Vergleich zwischen Niederdruckkalibrierung und linearem Verlauf (links) und das Verhältnis beider Verläufe im Druckbereich der freien Anströmung des HEG (rechts).

Autors mit einem dem Problem angepassten Drucksensor ${ }^{7}$ bestimmt. Der Messbereich dieses Aufnehmers ist 1000 Pa und dient zum einen der Niederdruckkalibrierung der Sensoren für die Messung des statischen Drucks, und zum anderen gibt er den Hintergrunddruck in der Messstrecke vor dem Versuch an. Die Nullpunktauflösung dieses Sensors liegt bei $0.5 \mathrm{~Pa}$. Alle Messstreckensensoren werden vor Versuchsbeginn auf Null abgeglichen, d.h. der Drucknullpunkt besitzt einen Fehler von maximal 0.5 Pa. Alle Werte für die Druckmessungen haben diesen systematischen Nullpunktsfehler. Bei den Messungen des statischen Druckes in dieser Arbeit war dieser Messaufnehmer nicht vorhanden. Der Messkammerhintergrunddruck wurde mit einem Sensor ${ }^{8}$ bestimmt, der einen viel größeren Messbereich hat. Hier liegt der Nullpunktsfehler bei $10 \mathrm{~Pa}$. Bei linearer Sensitivität der Aufnehmer und Druckwerten von $50 \mathrm{kPa}$, wie sie auf den Modelloberflächen typischerweise vorkommen, kann dieser Fehler vernachlässigt werden. Bei den kleinen Drücken für die statische Druckmessung muss dieser Fehler Berücksichtigung finden, da, wie schon gezeigt, die Annahme einer Linearität hier nicht mehr gilt und ein Referenzwert für die genaue Umwandlung der gemessenen Spannungen in einen Druckwert notwendig ist. Da bei den durchgeführten Experimenten dieser Referenzaufnehmer nicht vorhanden war, muss dieser systematische Fehler von $10 \mathrm{~Pa}$ in den einzelnen Messungen mit berücksichtigt werden.

\footnotetext{
${ }^{7}$ Modell CMR, Seriennummer: 44160712, Pfeiffer Vacuum GmbH, Asslar, Deutschland

${ }^{8}$ Modell 122AAx-01000PDS, Seriennummer: 78450G, MKS Instruments, Wilmington, MA, USA
} 


\section{Messtechniken und Modellinstrumentierung}

\subsection{Optische Methoden}

Für die optischen Untersuchungen der Strömung werden in dieser Arbeit zwei Verfahren verwendet. Die Hochgeschwindigkeits-Sichtbarmachung, basierend auf dem Toepler'schen Schlierenverfahren, wird benutzt, um die zeitliche Entwicklung des Strömungsaufbaus zu untersuchen. Stoßlagen, Grenz- und Scherschichten können bezüglich ihrer Lage vermessen werden. Die holographische Interferometrie wird als quantitative Methode im Einzelbildverfahren genutzt, um die relative Dichteänderung im Strömungsfeld zu bestimmen.

Beide Verfahren beruhen auf der Änderung der Ausbreitungsgeschwindigkeit des Lichtes in Abhängigkeit von der Dichte des Mediums, in dem es sich ausbreitet. Die folgende Betrachtung bezieht sich auf Licht einer Wellenlänge. Die Ausbreitungsgeschwindigkeit $c$ des Lichtes in einem gasförmigen Medium im Verhältnis zu seiner Ausbreitungsgeschwindigkeit im Vakuum $c_{0}$ ist über den Brechungsindex $n$ - in für diese Arbeit genügender Genauigkeit - folgendermaßen definiert:

$$
n(\rho)=\frac{c_{0}}{c}=1+\beta \frac{\rho}{\rho_{S}}=1+K \rho=1+\sum_{i}\left(\zeta_{i} K_{i}\right) \rho .
$$

Die Dichte $\rho_{S}$ bezieht sich auf einen bei Standardbedingungen ${ }^{1}$ definierten Wert. Der Skalierungsfaktor $\beta^{2}$ ist dimensionslos und abhängig von der Gasart und der Wellenlänge des Lichtes. Da die zu untersuchende Strömung chemischen Zustandsänderungen unterworfen ist, muss die Gladstone-Dale-Konstante $K$ abhängig von der Zusammensetzung des Gases über die Partialdichten $\zeta_{i}$ gebildet werden. Die dafür in dieser Arbeit benutzten GladstoneDale-Koeffizienten $K_{i}$ sind in Tab. 4.1 aufgelistet. Gleichung 4.1 zeigt, dass in einer Strömung mit veränderlicher Dichte der Brechungsindex eine Variable ist. Durch die Änderung der Ausbreitungsgeschwindigkeit werden die Lichtstrahlen an verschiedenen Orten unterschiedlich verzögert. Dadurch werden die Lichtstrahlen abgelenkt, und sie besitzen eine relative Phasenänderung zueinander. Die Ablenkung wird im Schlierenverfahren, die Phasenänderung im interferometrischen Verfahren ausgenutzt, um Rückschlüsse auf die Dichteänderung zu ziehen.

\begin{tabular}{|l|c|c|c|c|c|c|c|c|}
\hline Gaskomponente & $\mathrm{N}_{2}$ & $\mathrm{~N}$ & $\mathrm{O}_{2}$ & $\mathrm{O}$ & $\mathrm{NO}$ & $\mathrm{CO}_{2}$ & $\mathrm{CO}$ & $\mathrm{C}$ \\
$\mathrm{K}$ & 0.238 & 0.301 & 0.190 & 0.182 & 0.221 & 0.229 & 0.270 & 0.404 \\
\hline
\end{tabular}

Tabelle 4.1: Die in dieser Arbeit benutzten Gladstone-Dale-Konstanten. Die Werte sind von Alpher u. White (1959), Merzkirch (1987) und Kaye u. Laby (1986) publiziert.

\footnotetext{
${ }^{1} 0^{\circ} \mathrm{C}, 760 \mathrm{~mm} \mathrm{Hg}$

${ }^{2}$ In dieser Arbeit wird im Folgenden anstatt $\beta$ durchgehend der auf die Dichte bei Standardbedingungen bezogene Wert, die Gladstone-Dale-Konstante, $K$ benutzt.
} 


\subsubsection{Hochgeschwindigkeits-Sichtbarmachung}

Die für die Experimente verwendete Hochgeschwindigkeits-Sichtbarmachung basiert auf dem Schlierenverfahren. Die Entwicklung des Schlierenverfahrens wird Toepler (1864) und Foucault (1859) zugeschrieben. Neuere grundlegende Arbeiten stammen von Schardin (1934) und Holder u. North (1963). Eine Gesamtübersicht über Schlierenverfahren gibt Settles (2001). Da die Funktionsweise des Schlierenverfahrens ausführlich in der Literatur dargestellt ist, wird hier nur die technische Umsetzung des Aufbaus am HEG erläutert. Der Aufbau der Hochgeschwindigkeits-Sichtbarmachung ist in Abb. 4.1 schematisch dargestellt. Der optische Weg zum Einkoppeln des Laserlichts in die Messstrecke ist in Z-Form mit zwei großen sphärischen Hohlspiegeln ${ }^{3}$ ausgeführt, um Fehler durch sphärische Aberration in der Bildebene zu minimieren.

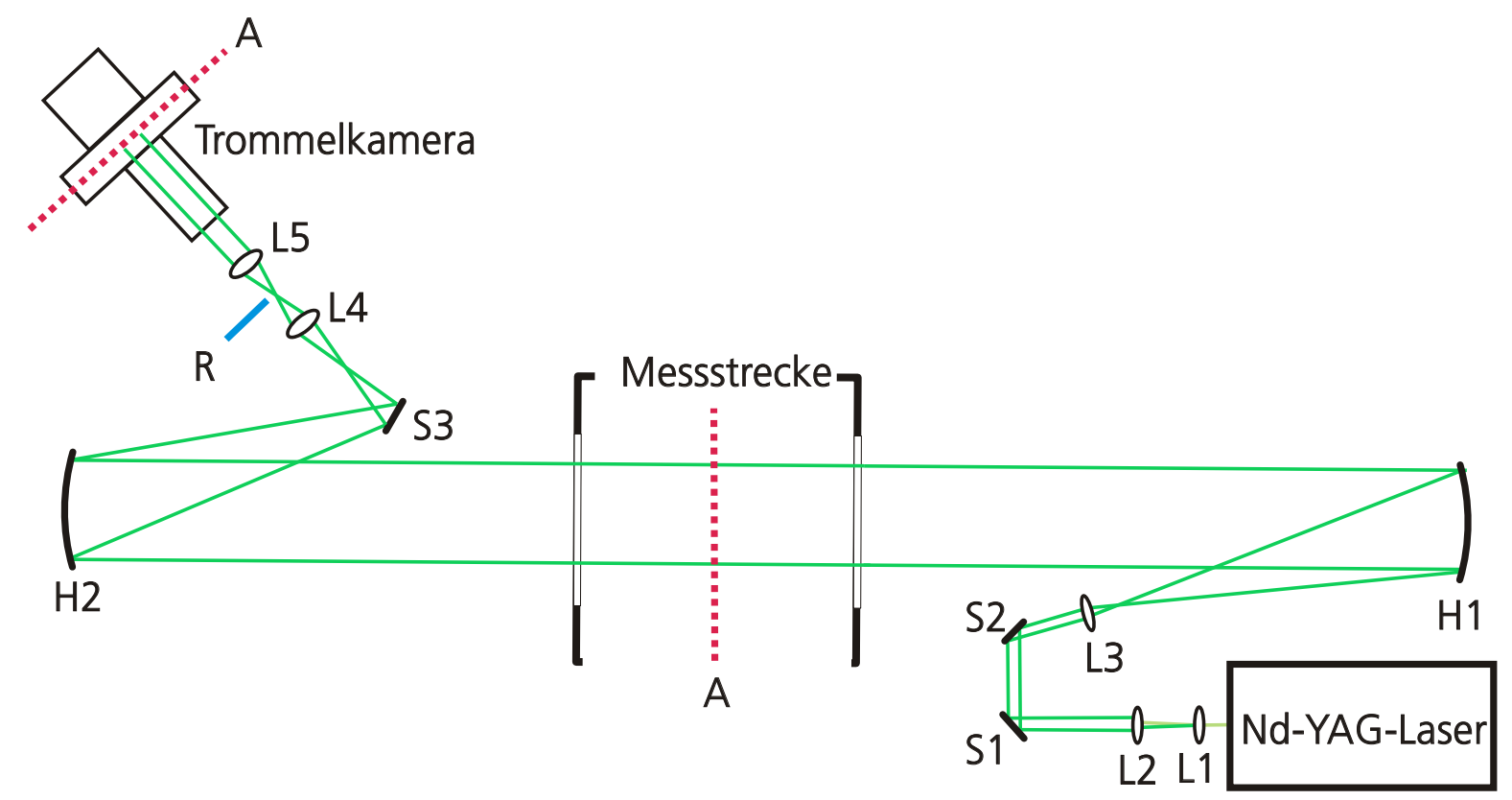

Abbildung 4.1: Schematischer Aufbau der Hochgeschwindigkeits-Sichtbarmachung. Der Aufbau nutzt das Schlierenverfahren. L: Linsen $\left(f_{1}=-30 \mathrm{~mm}, D_{1}=30 \mathrm{~mm}, f_{2}=300 \mathrm{~mm}, D_{2}=30 \mathrm{~mm}, f_{3}=\right.$ $\left.-50 \mathrm{~mm}, D_{3}=60 \mathrm{~mm}, f_{4}=120 \mathrm{~mm}, D_{4}=60 \mathrm{~mm}, f_{5}=90 \mathrm{~mm}, D_{5}=60 \mathrm{~mm}\right), \mathbf{S}$ : Spiegel $\left(D_{1}-\right.$ $\left.D_{3}=80 \mathrm{~mm}\right), \mathbf{H}$ sphärische Spiegel $\left(f_{1}-f_{2}=1500 \mathrm{~mm}, D_{1}-D_{2}=300 \mathrm{~mm}\right), \mathbf{R}$ : Schlierenkante, A:Abbildungsebene.

Als Lichtquelle wird ein diodengepulster Nd:YAG-Laser ${ }^{4}$ benutzt. Dieser erzeugt Lichtpulsfolgen mit variabler Anzahl und Pulsraten bis $50 \mathrm{kHz}$. Der Nd:YAG-Laser emittiert Licht der Wellenänge 532 nm (grün). Das vom Laser emittierte Licht wird mittels einer Teleskop-Optik

${ }^{3}$ Die verwendeten Hauptspiegel sind sphärische Hohlspiegel (SDH4300) der Firma B. Halle Nachfl. GmbH, Hubertusstraße 10, 12163 Berlin. Sie sind aus Duran 50 gefertigt und haben eine mittlere Abweichung von der Sphäre von $\lambda / 4$.

${ }^{4}$ Model 612 S-532-6000 von Lightwave Electronics, Soliton Laser und Messtechnik GmbH, Talhofstr. 32, 82205 Gilching [www.soliton-gmbh.de] 


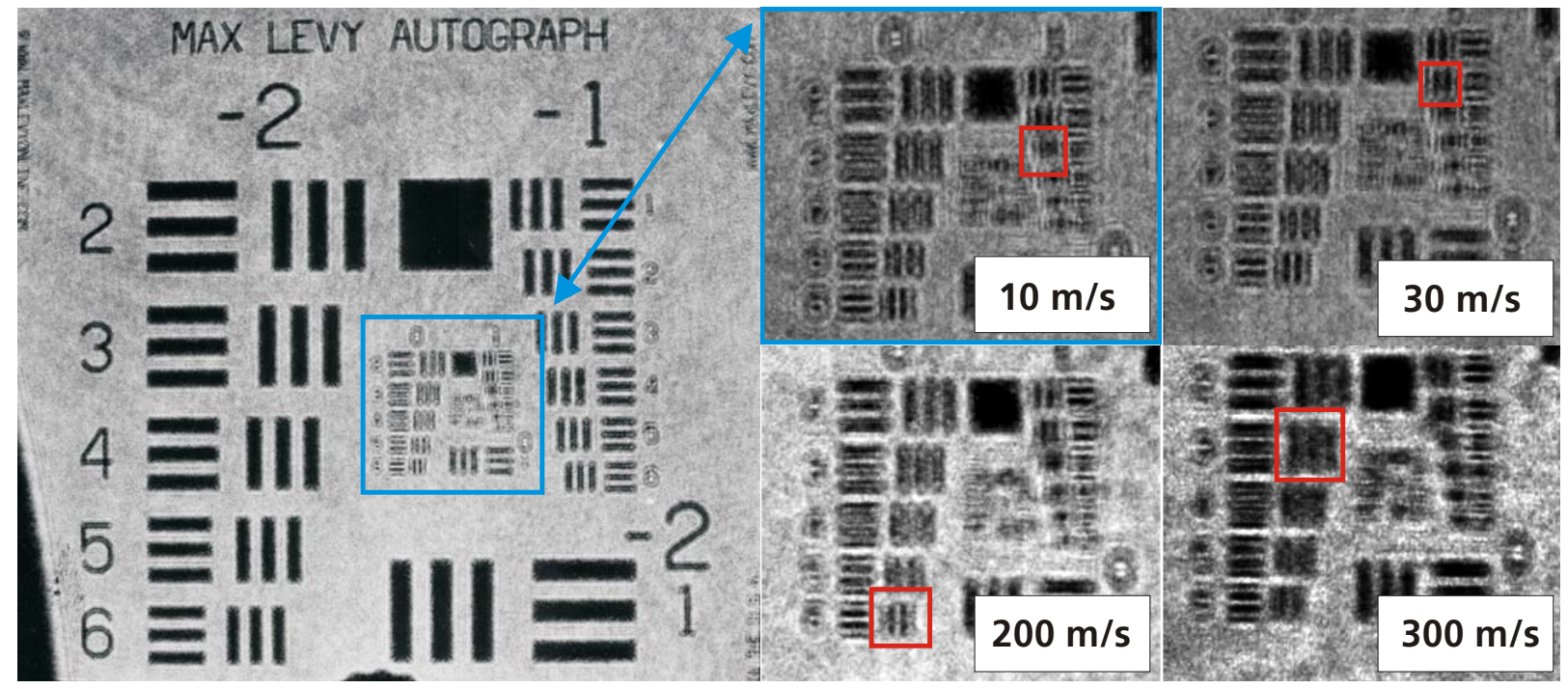

Abbildung 4.2: Darstellung des USAF-Testbildes bei verschiedenen Drehgeschwindigkeiten der Trommelkamera und Frequenzen/Pulsbreiten des Nd:YAG-Lasers.

$(\mathrm{L} 1, \mathrm{~L} 2)$ auf $30 \mathrm{~mm}$ Durchmesser aufgeweitet, umgelenkt $(\mathrm{S} 1, \mathrm{~S} 2)$ und dann über die Linse L3 im Brennpunkt des großen sphärischen Spiegels H1 fokussiert. Das Licht durchläuft, aufgeweitet auf $300 \mathrm{~mm}$, die Messstreckenfenster und wird von dem zweiten großen sphärischen Hauptspiegel H2 über den Spiegel S3 fokussiert. In dem nach der Linse L4 enstehenden Brennpunkt wird die Schlierenkante R platziert. Die Abbildungsebene in der Messstrecke wird mit Hilfe der Linse L5 auf die Filmebene in der Trommelkamera abgebildet. Die Trommelkamera transportiert in ihrem Inneren einen Film ${ }^{5}$ von $1 \mathrm{~m}$ Länge. Diese Länge entspricht dem inneren Umfang der Trommel. Die maximale Drehgeschwindigkeit beträgt $300 \mathrm{~m} / \mathrm{s}$. Da sich unter der Belichtung der Film kontinuierlich bewegt, bestimmt die Pulsbreite des Lasers die Verschlusszeit. Der Film liegt als Ring im Inneren der Trommel. Weil sich die Lage des Startbildes nicht festlegen lässt, kann es passieren, dass ein Bild auf die Schnittkante des Films belichtet wird. Dieses Bild kann bei der Auswertung nicht berücksichtigt werden.

Die zeitliche Dauer der einzelnen Lichtpulse steigt mit der benutzten Wiederholrate des Lasers und liegt im Bereich von 43 ns bei $1 \mathrm{kHz}$ bis $155 \mathrm{~ns}$ bei $50 \mathrm{KHz}$. Bei der maximalen Drehgeschwindigkeit und Frequenz des Systems führt dies zu einem Wegversatz des Films von $46.5 \mu \mathrm{m}$ während der Belichtungszeit. Diese Werte schwanken im Versuchsbetrieb. Um die echte Auflösung des Systems zu bestimmen, wurde ein Auflösungstestbild ${ }^{6}$ in der Messstrecke des HEG installiert und auf Film belichtet. Der ganze sichtbare Durchmesser der Fenster $(300 \mathrm{~mm})$ wurde auf den Film (12mm) abgebildet. Der Abbildungsmaßstab von Messstrecke zu Film beträgt somit 1:25. Das Testbild, das zentriert im Fenster platziert wurde, ist quadratisch und besitzt eine Kantenlänge von $7.62 \mathrm{~cm}$. Auf dem Testbild sind horizontale und vertikale Streifen in unterschiedlichen Abständen vorhanden. Können die Streifen nicht mehr unterschieden werden, ist die optische Grenze der Auflösung des

\footnotetext{
${ }^{5}$ Das Filmmaterial für die Hochgeschwindigkeitskamera ist typischerweise Kodak T-MAX 100 (SchwarzWeiss-Film).

${ }^{6}$ Target USAF 1951 1X (3x3 Zoll) hergestellt von Max Levy Autograph, Inc., 220 West Roberts AVE, Phila., PA, [www.maxlevyonline.com].
} 
Systems bestimmt. In Abb. $\mathbf{4 . 2}$ ist das Testbild zusammen mit den Einzelmessungen dargestellt. Die Bewegunsrichtung des Filmes liegt in der horizontalen Ebene. Dies ist auch an den Strichmustern zu erkennen. Die vertikalen Streifen verlaufen und sind nicht mehr unterscheidbar, während es die horizontalen Streifenpaare noch sind. Die Ergebnisse sind in Tab. 4.2 angegeben. Die beste Auflösung beträgt $63 \mathrm{lp} / \mathrm{mm}$ (Linienpaare pro Millimeter) bei kürzester Pulsbreite und kleinster Drehgeschwindigkeit der Trommel. Diese Begrenzung ist nicht durch die optische Auflösung des Linsensystemes (ca. 80 lp/mm) bedingt, sondern eher durch die Beugungseffekte, die bei der Verwendung des kohärenten Laserlichts entstehen.

\begin{tabular}{|c|c|c|c|c|}
\hline $\begin{array}{l}\text { Drehgeschwindigkeit } \\
\text { der Filmtrommel }\end{array}$ & $\begin{array}{l}\text { Wegversatz während der Belichtung } \\
\text { auf der Filmebene }\end{array}$ & $\begin{array}{c}\text { Wegänderung während der Belichtung* } \\
\text { bezogen auf die Abbildung der Messstrecke }\end{array}$ & $\begin{array}{c}\text { Gemessene } \\
\text { Auflösung auf der Filmebene }\end{array}$ & $\begin{array}{l}\text { Gemessene } \\
\text { Auflösung }\end{array}$ \\
\hline $\mathrm{m} / \mathrm{s}$ & $\mu \mathrm{m}$ & $\mathrm{mm}$ & $\mathrm{lp} / \mathrm{mm}$ & Ip/mm \\
\hline 10 & 1.55 & 0.0062 & 63.00 & 2.52 \\
\hline 30 & 4.65 & 0.0186 & 56.00 & 2.24 \\
\hline 200 & 31.00 & 0.1240 & 44.50 & 1.78 \\
\hline 300 & 46.50 & 0.1860 & 31.50 & 1.26 \\
\hline
\end{tabular}

Tabelle 4.2: Messung der optischen Auflösung der Hochgeschwindigkeits-Sichtbarmachung.

Bei der höchsten Trommeldrehzahl der Kamera und längster Belichtungszeit reduziert sich die gemessene optische Auflösung bis auf $31.5 \mathrm{lp} / \mathrm{mm}$. Diese Kombination stellt den ungünstigsten Fall dar und kann deshalb als untere Grenze der Auflösung betrachtet werden. Für die bei den Messungen benutzten Kombinationen zwischen Frequenz und Drehzahl liegt die Auflösung bei $2 \mathrm{lp} / \mathrm{mm}$, bezogen auf die Bildebene in der Messstrecke. Damit liegt der Abbildungsfehler für eine geometrisch bestimmte Größe, wie z.B eine Stoßlage, bei ca. $0.25 \mathrm{~mm}$.

\subsubsection{Holographische Interferometrie}

\section{Bestimmung der Phasenverschiebung durch Interferenz}

Die Dichte eines gasförmigen Mediums bestimmt die Ausbreitungsgeschwindigkeit des Lichtes. Eine Änderung der Dichte führt zu einer Geschwindigkeitsänderung, und diese bedingt eine Phasenverschiebung der sich ausbreitenden Welle bezogen auf einen Referenzzustand. Mittels Interfenzerscheinungen lässt sich diese Phasenverschiebung bestimmen und zur quantitativen Dichtebestimmung nutzen. Das Prinzipbild in Abb. $\mathbf{4 . 3}$ zeigt zwei Lichtstrahlen 1 und 2, die auf unterschiedlichem Wege den Punkt $P$ auf dem Schirm erreichen. Ob sich beide Lichtstrahlen im Punkt $P$ verstärken oder auslöschen (positiv oder negativ interferieren), hängt von der Phasendifferenz beider Strahlen im Punkt $P$ ab. Durchlaufen beide Strahlen ihre Pfade in identischen Medien, definiert diese Phasendifferenz den Längenunterschied des geometrischen Weges. Verfahren zur Längenmessung basieren auf diesem Prinzip. Befindet sich ein Strahl in einem anderen Medium $n_{2}$ mit unterschiedlichem Brechungsindex $n_{2}<>n_{1}$, wird die effektive oder optische Weglänge geändert, und die Ordnung der Interferenz im Punkt $P$ ändert sich.

Ist die Änderung des optischen Weges ein ganzzahliges Vielfaches $N \lambda$ der Wellenlänge des 
Lichtstrahles, wird die Ordnung der Interferenz im Punkt $P$ um $N$ erhöht. Eine Erhöhung des Brechungsindex $n_{2}$ von $n_{1}$ aus erniedrigt die Ausbreitungsgeschwindigkeit des Lichstrahles von $c_{0} / n_{1}$ auf $c_{0} / n_{2}$. Die zusätzliche Zeit, die der Lichtstrahl 2 braucht, um im Punkt P anzukommen, beträgt:

$$
\Delta t=\frac{L}{c_{2}}-\frac{L}{c_{1}}=\frac{L}{c_{0}}\left(n_{2}-n_{1}\right)
$$

Damit ergibt sich die Änderung des optischen Weges zu

$$
\Delta L=c_{0} \Delta t=L\left(n_{2}-n_{1}\right) .
$$

Die Änderung der Ordnung ist dann durch

$$
N=\frac{\Delta L}{\lambda}=\frac{L}{\lambda}\left(n_{2}-n_{1}\right)=\frac{K L}{\lambda}\left(\rho_{2}-\rho_{1}\right)=\frac{\Delta \phi}{2 \pi}
$$

gegeben, wobei die Beziehung 4.1 benutzt wurde und $\Delta \phi$ die sich ergebene Phasenverschiebung ist. Besitzen die Strahlen in Abb. 4.3 eine endliche Ausdehnung und bilden sie ein zweidimensionales Bild ab, ergibt sich dann:

$$
\Delta \phi(x, y)=\frac{1}{2 \pi \lambda} \int_{z_{1}}^{z_{2}}\left(\rho_{\infty} K_{\infty}-\rho(x, y, z) \sum_{i=1}^{N S} K_{i} \zeta_{i}(x, y, z)\right) d z
$$

Die Messmethode ist sehr empfindlich. Optische Wegänderungen im Bereich der Wellenlänge des verwendeten Lichtes (400 nm - 800 nm) erzeugen messbare Phasenverschiebungen. Als Konsequenz existieren hohe Anforderungen an die Güte der benutzten optischen Komponenten wie Linsen, Spiegel und Messstreckenfenster. Auch kleinste Unzulänglichkeiten werden als Phasenverschiebungen mitgemessen. Die für die Messungen benutzte holographische Interferometrie misst die Phasenverschiebung nicht wie in Abb. 4.3 dargestellt direkt, sondern speichert nur die Lichtinformationen des Objektstrahles (z.B Strahl 1) vor und während des Versuches. Diese beiden Bilder werden dann zur Interferenz gebracht. Damit

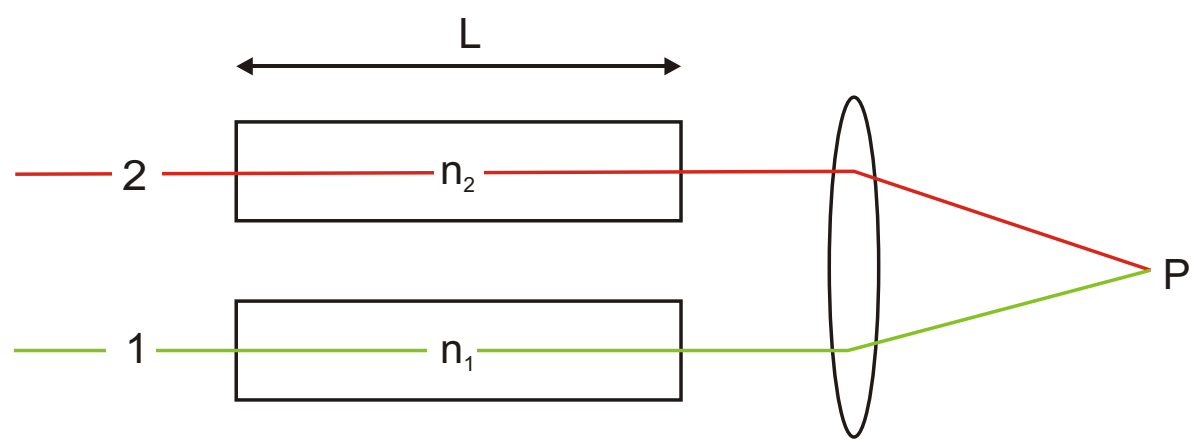

Abbildung 4.3: Prinzip der interferometrischen Messung des Brechungsindexes. 
können alle Störungen im Strahlengang, die nicht von einer Dichteänderung in der Messstrecke hervorgerufen werden, unterdrückt werden. Um die gesamten Lichtinformationen im Strahlengang zu speichern, kann die Aufzeichnung nicht photographisch erfolgen; eine holographische Speicherung ist notwendig.

Mit Hilfe der Holographie ist es möglich, dreidimensionale Wellenfelder auf zweidimensionalen Speichern, wie Photoplatten, aufzuzeichnen. Die gespeicherten Wellenfelder lassen sich so rekonstruieren, als seien die Wellen durch die Speicherung überhaupt nicht unterbrochen worden, sondern hätten sich unbeeinflusst ausgebreitet. Alle Aufnahmemedien, die für eine Speicherung von Strahlungsfeldern in Frage kommen, z.B. photographische Platten, reagieren nur auf die während ihrer Belichtung pro Flächeneinheit einfallende Lichtenergie. Ist

$$
\Psi_{G}(x, y, t)=A_{G}(x, y) e^{j\left[\varphi_{G}(x, y)+\omega t\right]}
$$

die elektrische Feldstärke einer aufzunehmenden Gegenstandswelle mit der Amplitude $A_{G}$, der Phase $\varphi_{G}$ und der Kreisfrequenz $\omega$ in einem Punkt $(x, y)$ der Registrierfläche $F$ zur Zeit $t$, so beträgt die Energiedichte dort

$$
W_{\text {Photographie }}=\frac{\Psi_{G} \Psi_{G}^{*}}{Z} t_{B}=\frac{A_{G}^{2}(x, y)}{Z} t_{B},
$$

wobei $t_{B}$ die Belichtungszeit der Aufnahme und $Z$ der Wellenwiderstand des Raumes ist, in dem sich die Welle ausbreitet. $\Psi_{G}^{*}(x, y, t)$ ist die zu $\Psi_{G}(x, y, t)$ konjugiert komplexe Größe. Die Energiedichte, die man bei dieser photographischen Speicherung aufzeichnet (Abb. 4.4 links), enthält nach Gleichung 4.3 keine Information über die Phasenverteilung $\varphi_{G}(x, y)$ der Gegenstandswelle mehr und deshalb keine Information über die Dichteänderungen, die sie durchlaufen hat. Die photographische Speicherung ist für die holographische Interferometrie nicht verwendbar.

Überlagert man der Gegenstandswelle $\Psi_{G}(x, y, t)$ nach Abb. 4.4 (Mitte) einen zu ihr kohärenten Hintergrund in Form einer zweiten kohärenten Bezugswelle

$$
\Psi_{B}(x, y, t)=A_{B}(x, y) e^{j\left[\varphi_{B}(x, y)+\omega t\right]}
$$

gleicher Frequenz, deren Wellenfronten auf der Fläche $\mathrm{F}$ eine andere Form, also andere Phasenverteilung $\varphi_{B}(x, y)$ haben als $\Psi_{G}(x, y, t)$, dann ergibt sich auf dem photographischen Medium die Energiedichte

$$
\begin{aligned}
W_{\text {Holographie }} & =\frac{t_{B}}{Z}\left(\Psi_{G}+\Psi_{B}\right)\left(\Psi_{G}^{*}+\Psi_{B}^{*}\right) \\
& =\frac{t_{B}}{Z}\left(A_{G}^{2}+A_{B}^{2}+2 A_{G} A_{B} \cos \left(\varphi_{G}-\varphi_{B}\right)\right]
\end{aligned}
$$

Bei dieser sogenannten holographischen Aufzeichnung bleibt die Phasendifferenz zwischen der Gegenstandswelle und der Bezugswelle erhalten. Je nach Phasendifferenz $\left(\varphi_{G}-\varphi_{B}\right)$ 

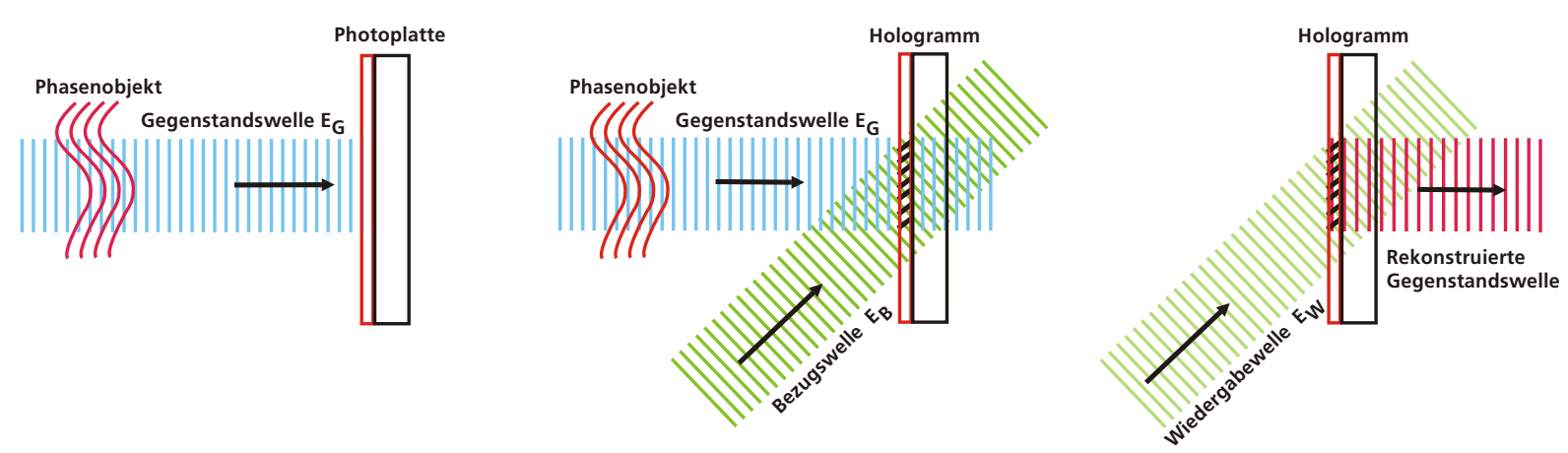

Abbildung 4.4: Schematische Darstellung der photographische Speicherung (links), der holographischen Speicherung (mitte) und der holographischen Rekonstruktion (rechts).

beider Wellen entstehen auf der Fläche F Regionen hoher oder geringer Energiedichte, die zu starker oder schwacher Schwärzung des photographischen Mediums führen. Die Amplituden- und Phaseninformation über beide Wellen ist in dieser Interferenzfigur enthalten und kann in dieser Form photographisch festgehalten werden. Diese Interferenzaufnahme bezeichnet man als Hologramm.

Die Wiedergabe eines Hologramms, also die Rekonstruktion der aufgenommenen Gegenstandswelle, ist dadurch möglich, dass man entsprechend Abb. $\mathbf{4 . 4}$ auf der rechten Seite das Hologramm mit einer kohärenten Wiedergabewelle

$$
\Psi_{W}(x, y, t)=A_{W}(x, y) e^{j\left[\varphi_{W}(x, y)+\omega t\right]}
$$

durchstrahlt. Hier wird eine Wiedergabewelle erzeugt, die der Bezugswelle gleicht. Beim Durchtritt durch das Hologramm erfährt die Wiedergabewelle $\Psi_{W}(x, y, t)$ eine Dämpfung, die von der Schwärzung des Hologramms abhängig ist und daher von Ort zu Ort auf dem Hologramm entsprechend der aufgenommenen Interferenzfigur variiert. Dadurch entsteht hinter der Hologrammfläche eine Intensitätsverteilung, die bis auf Feinheiten eine getreue Nachbildung der Gegenstandswelle bei der Aufnahme ist. So wie sich das Lichtfeld bei der Aufnahme aus der Überlagerung von Gegenstands- und Bezugswelle zusammensetzt, lässt es sich bei der Rekonstruktion in zwei entsprechende Wellen zerlegen: Eine Welle, die mit der Bezugswelle übereinstimmt und die durch den Mittelwert der Schwärzung gleichmäBig gedämpft wird, und eine zweite Welle, deren Amplituden- und Phasenverteilung mit derjenigen der Gegenstandswelle übereinstimmt: die rekonstruierte Gegenstandswelle.

Bei der Einstrahlung der Wiedergabewelle $\Psi_{W}(x, y, t)$ ergibt sich auf der rückwärtigen Hologrammfläche eine elektrische Feldstärke proportional zu

$$
\begin{aligned}
\Psi_{W} \cdot W_{\text {Holographie }} & \sim A_{W} e^{j\left(\varphi_{B}+\omega t\right)}\left(A_{G}^{2}+A_{B}^{2}\right) \\
& +A_{G} A_{B} A_{W} e^{j\left(\varphi_{G}+\omega t\right)} \\
& +A_{G} A_{B} A_{W} e^{j\left(2 \varphi_{B}-\varphi_{G}+\omega t\right)}
\end{aligned}
$$

wobei $W_{\text {Holographie }}$ nach Gleichung 4.4 eingesetzt wurde. Der erste Term (4.5) ist die gedämpfte Wiedergabewelle, er entspricht der Bezugswelle. Der zweite Term (4.6) ist bis auf 


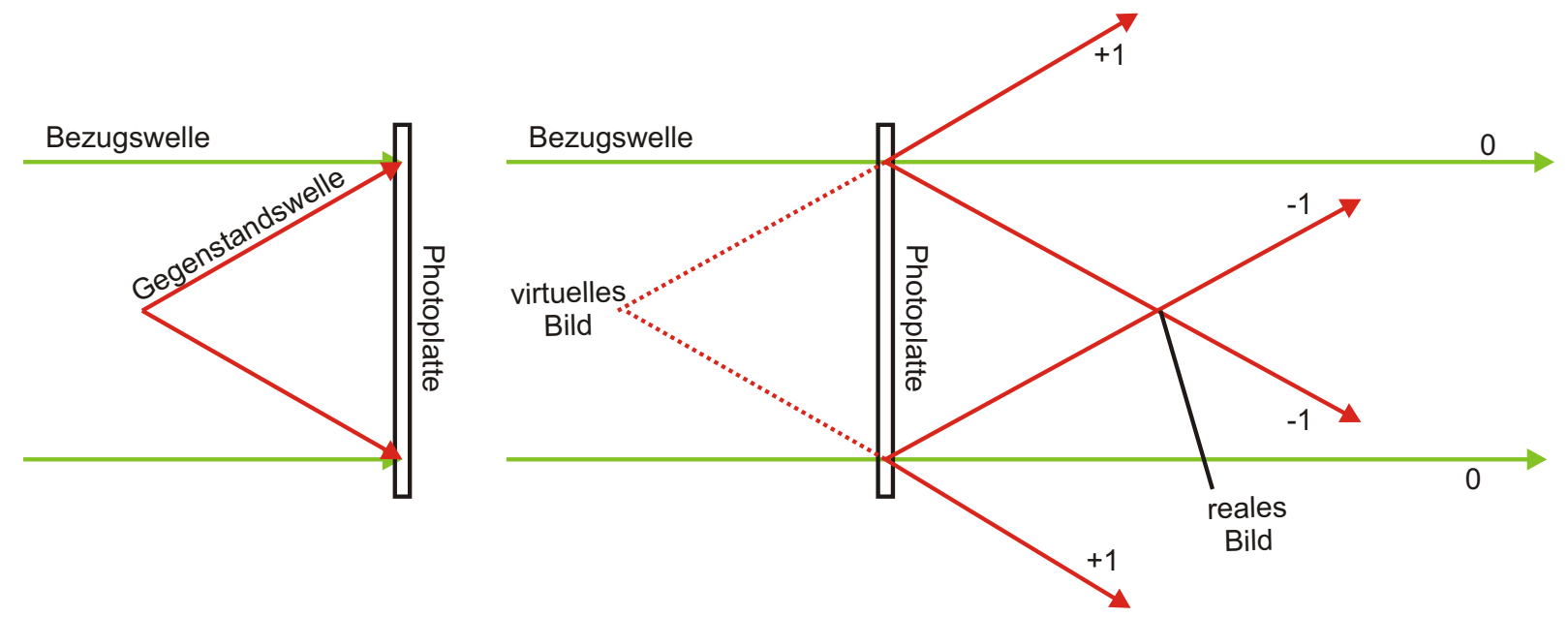

Abbildung 4.5: Schema der holographischen Bildrekonstruktion.

einen konstanten Amplitudenfaktor identisch mit der Gegenstandswelle $\Psi_{G}$ : Durch eine mit der Bezugswelle übereinstimmende Wiedergabewelle wird die Gegenstandswelle getreu rekonstruiert. Der dritte Term (4.7) stellt eine neu hinzugekommene Welle dar. Auch sie enthält $A_{G}$ und $\varphi_{G}$ und damit die gesamte Information über die Gegenstandswelle. $\varphi_{G}$ tritt hier aber mit negativem Vorzeichen aus weshalb man diese Welle die konjugierte rekonstruierte Welle nennt. Die konjugierte Welle verlässt wegen ihrer Phase $\left(2 \varphi_{B}-\varphi_{G}\right)$ das Hologramm unter anderer Richtung als die eigentliche rekonstruierte Welle.

Die Entstehung der rekonstruierten Wellen lässt sich noch auf andere Weise beschreiben: Das Hologramm kann als Beugungsgitter mit örtlich variabler Gitterkonstante betrachtet werden, an dem die einfallende Wiedergabewelle gebeugt wird. Dies ist in Abb. $\mathbf{4 . 5}$ dargestellt. Der geradeaus durchgehende und dabei gleichmäßig geschwächte Anteil ist die ungebeugte Ordnung, direkte und konjugierte Welle sind die beiden Wellen der ersten Beugungsordnung. Der Beugungswinkel ist der Gitterkonstanten umgekehrt proportional.

\section{Aufbau der holographischen Interferometrie}

Die Gegenstandswelle in obiger Betrachtung wird im realen Versuchsaufbau am HEG mittels eines Riesenimpulslasers, eines Rubinlasers ${ }^{7}$, erzeugt. Das vom Rubinlaser emittierte Licht $\left(\lambda_{\text {Rubin }}=694.3 \mathrm{~nm}\right)$ wird mittels einer Aufweitungsoptik auf einen Durchmesser von $30 \mathrm{~mm}$ gebracht. Danach durchläuft das Licht einen Strahlteiler, der das Licht in zwei Strahlen auftrennt. Ein Lichtstrahl, der die Gegenstandswelle bildet, wird weiter aufgeweitet und leuchtet den sphärischen Hohlspiegel (s. Abb. 4.1, S. 40) von 300 mm Durchmesser aus.

Die so erzeugte parallele Gegenstandswelle, die durch die beiden Messstreckenfenster läuft, wird dann mittels des zweiten Hohlspiegels über eine fokussierende Linse auf die Hologrammplatte abgebildet. Die Gegenstandswelle kann mittels eines Graukeils in ihrer Intensität reguliert werden. Dies dient der Kontrasterhöhung der aufgenommenen Hologramme. In Abb. 4.6 ist der Strahlengang schematisch dargestellt. Wie hier eingezeichnet, wird

\footnotetext{
${ }^{7}$ Model xxx, Innolas, $x x x$
} 
der zweite durch den Strahlteiler erzeugte Lichtstrahl um die Messstrecke herumgeführt und fällt mittels mehrerer Umlenkungen als Bezugswelle ebenfalls auf die Hologrammplatte.

Beide Wellen, Gegenstands- und Bezugswelle, interferieren auf der Hologrammplatte miteinander. Das Interferenzmuster wird während der Belichtungsdauer auf der Hologrammplatte gespeichert. Die Belichtungsdauer entspricht hier der Pulsdauer des Rubinlasers, die ungefähr $t_{B(\text { Rubin })}=10$ ns beträgt. Der Zusammenhang zwischen dem Winkel der einfallenden Gegenstandswelle zur Bezugswelle $\beta$ und der erzeugten Interferenzstreifenbreite $F_{l}$ oder Ortsfrequenz auf dem Hologramm wird durch

$$
\beta=\arcsin \left(F_{l} \cdot \lambda\right)
$$

wiedergegeben. Bei in der Praxis benutzten Aufnahmewinkeln zwischen Gegenstandswelle und Bezugswelle in der Größenordnung von $30^{\circ}$ wird ein hochauflösendes photographisches Medium benötigt. Für die Experimente wurden ausschließlich Hologrammplatten (AGFA 8E75 HD) mit einer Auflösung von 5000 lp/mm benutzt. Gleichung 4.8 besagt auch, dass es möglich ist, unter verschiedenen Aufnahmewinkeln verschiedene Hologramme auf der photoaktiven Schicht der Hologrammplatte zu speichern. Bei der Messung im HEG werden zwei Hologramme auf der Hologrammplatte gespeichert. Eine Belichtung wird kurz

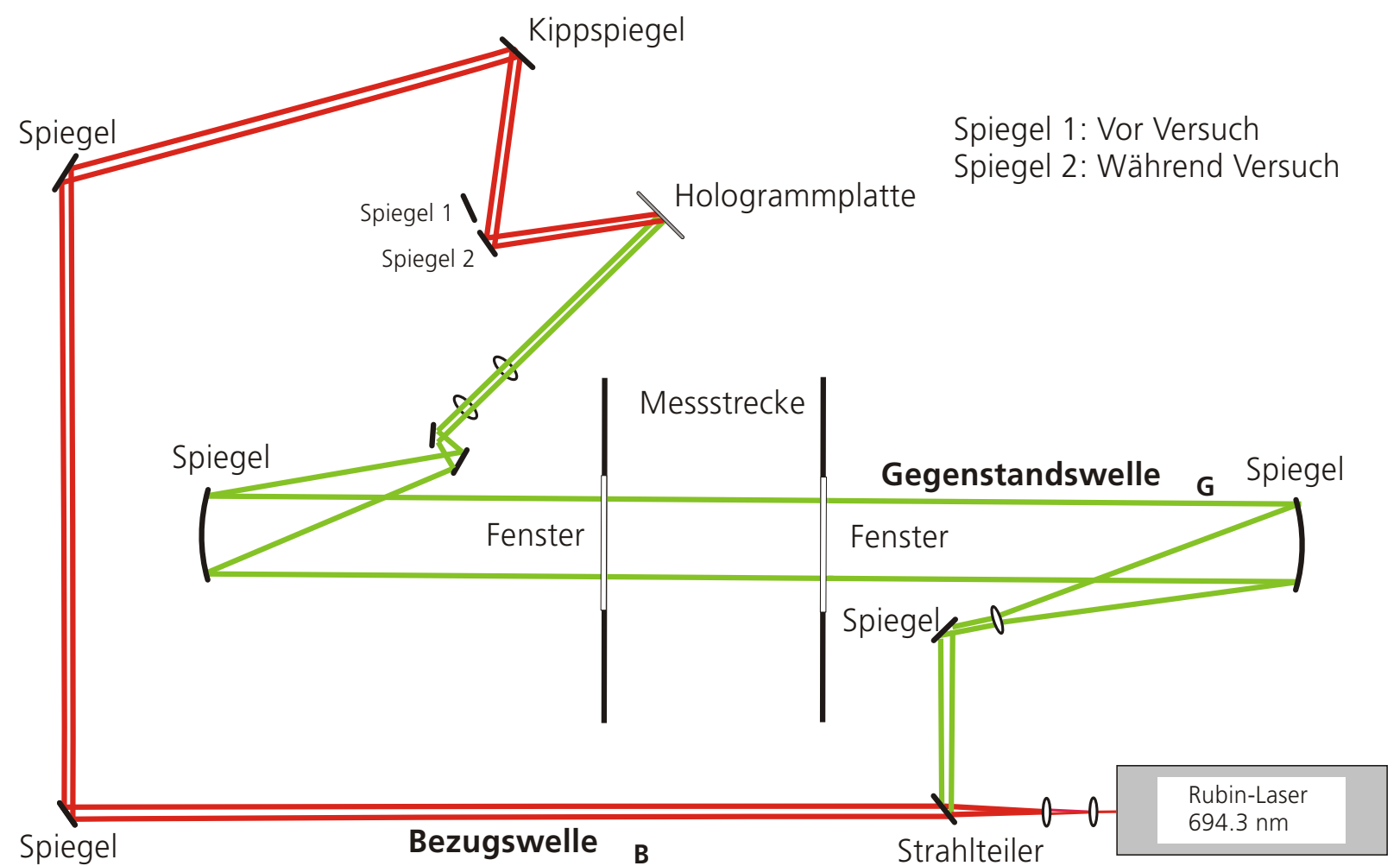

Abbildung 4.6: Schematische Ansicht des Strahlenganges für die Aufnahme der Hologramme am HEG. Die Gegenstandswelle (rot) und die Bezugswelle (grün) sind für eine bessere Übersicht farblich unterschieden, es handelt sich aber um Licht einer Wellenlänge. 

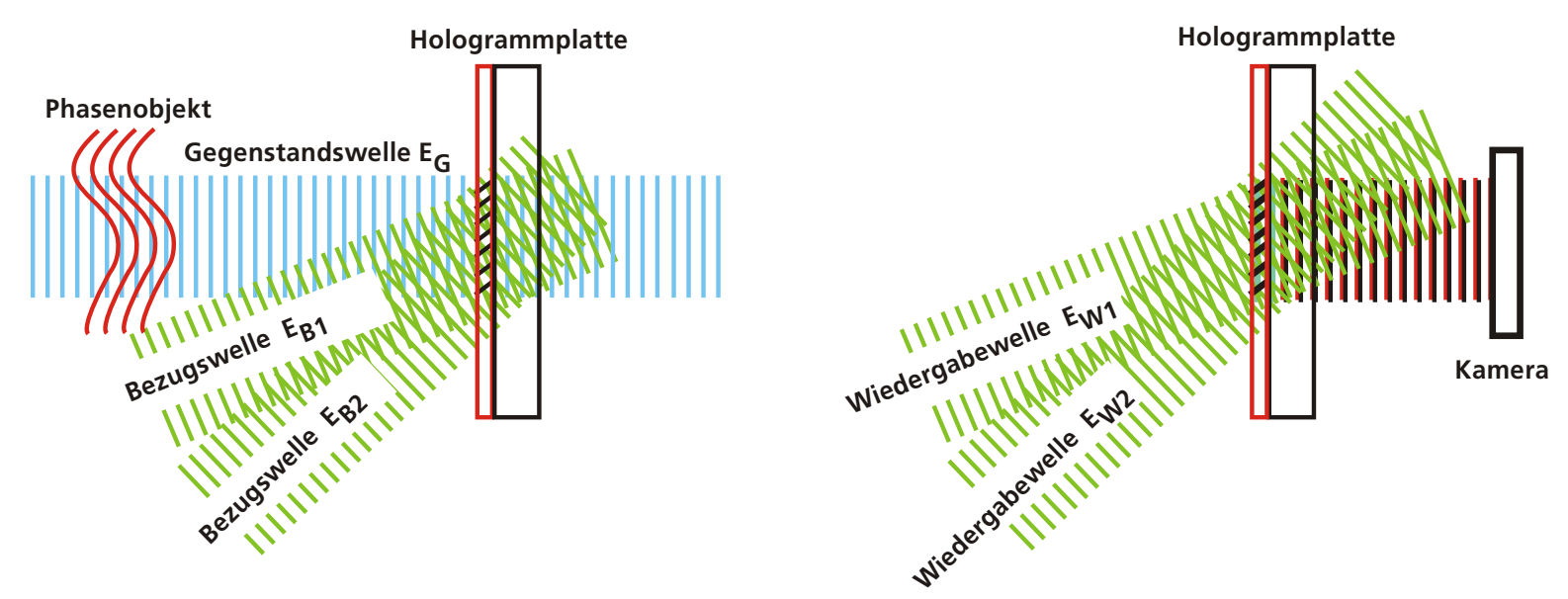

Abbildung 4.7: Prinzip der Aufnahme und Rekonstruktion bei der holographischen Interferometrie: Durch Überlagerung der Gegenstandswelle mit zwei Bezugswellen (links) vor $\left(E_{B 1}\right)$ und während des Versuchs $\left(E_{B 2}\right)$ werden zwei Hologramme auf der Hologrammplatte gespeichert. Diese werden bei der Rekonstruktion (rechts) mit zwei geometrisch ähnlichen Wiedergabewellen rekonstruiert und zur Interferenz in der Kameraebene gebracht.

vor dem Versuch durchgeführt, und nach dem Drehen des Kippspiegels (Abb. 4.6) wird ein zweites Interferenzmuster während des Versuches unter einem anderen Winkel der Bezugswelle auf die Hologrammplatte belichtet.

Die Abb. 4.7 stellt dieses im Schema dar. Nach der chemischen Verarbeitung der Hologrammplatte wird sie mittels zweier Wiedergabewellen $\Psi_{W 1}$ und $\Psi_{W 2}$ durchleuchtet. Jede der Wiedergabewellen erzeugt ein System von Wellen nach Gleichung 4.7 hinter der Hologrammplatte. Die beiden rekonstruierten Gegenstandswellen werden dann so einjustiert, dass sie hinter der Hologrammplatte auf einer Kamera interferieren. Die Aufnahme und die Rekonstruktion in Kombination wird als Verfahren der holographischen Interferometrie bezeichnet. Die rekonstruierten Gegenstandswellen $\Psi_{R 1}$ und $\Psi_{R 2}$ definieren sich der obigen Notation folgend als

$$
\Psi_{R 1}(x, y)=A_{G 1} A_{B 1} A_{W 1} e^{j\left(\varphi_{G 1}+\omega\right)} \text { und } \Psi_{R 2}(x, y)=A_{G 2} A_{B 2} A_{W 2} e^{j\left(\varphi_{G 2}+\omega\right)} .
$$

Dann ergibt sich folgende Energiedichte auf der Kamera:

$$
\begin{aligned}
W_{H I} & =\left(\Psi_{R 1}+\Psi R 2\right)\left(\Psi_{R 1}^{*}+\Psi_{R 2}^{*}\right) \\
& =\left(A_{G 1} A_{B 1} A_{W 1}\right)^{2}+\left(A_{G 2} A_{B 2} A_{W 2}\right)^{2} \\
& +2 A_{G 1} A_{B 1} A_{W 1} A_{G 2} A_{B 2} A_{W 2} \cos \left(\varphi_{G 1}-\varphi_{G 2}\right)
\end{aligned}
$$

Wie man aus dem letzten Term in 4.9 ersehen kann, zeigt das Interferenzbild auf der Kamera dann in jedem Punkt die Phasenverschiebung des Lichtes während des Versuchs $\varphi_{G 2}$ relativ zu einer Phasenverschiebung $\varphi_{G 1}$, die vor dem Versuch in der ersten belichteten Gegenstandswelle enthalten war. Aus Gleichung 4.9 wird auch deutlich, dass es vorteilhaft 
ist, das Verhältnis der Beleuchtungsstärke von der Bezugswelle zur Gegenstandswelle klein zu halten. Damit wird der Amplitudenterm, der die Intensität des Phasenkontrastes steuert, nur von der Beleuchtungsstärke der Bezugswelle bestimmt. Amplitudenänderungen in der Gegestandswelle machen sich nicht mehr so stark im Phasenkontrast bemerkbar. Die von der Kamera aufgezeichnete Beleuchtungsstärke $I(x, y)$ auf jedem Pixel ist dann zusammengefasst definiert durch

$$
I(x, y)=I_{0}[1+m(x, y) \cos (\Delta \varphi(x, y))]
$$

wobei $m$ der Streifenkonstrast, $I_{0}$ die Hintergrundintensität und $\Delta \varphi=\left(\varphi_{G 1}-\varphi_{G 2}\right)$ die Phasenverschiebung in jedem Punkt der Kameraebene ist. Um diese Streifenbilder auszuwerten, können numerische Verfahren, wie die Fouriertransformation, benutzt werden (Vest (1979), Houwing (1999)); trotzdem ist die Kenntnis, ob die Phasenverschiebung beim Überschreiten einer Periode abnimmt oder zunimmt, vorauszusetzen. Um diese aufwendige Bearbeitung zu umgehen, wird die Methode der Phasenkontrolle, das Phasenschrittverfahren, seit Ende der 70er Jahre eingesetzt (Trolinger (1979)). Durch die schnelle Zunahme der verfügbaren Rechnerleistung wurde die Entwicklung dieses Phasenschrittverfahrens, das auch als quasi-heterodyne Interferometrie bezeichnet wird (Dändliker u. Thalmann (1985)), vorangetrieben. Bei der in dieser Arbeit benutzten Phasenschritt-Technik wird der optische Weg einer der Wiedergabewellen in Bild 4.7b mittels eines piezoelektrischen Spiegels verlängert und der Phasenverschiebung ein bekannter Wert aufaddiert. In der Praxis werden drei individuelle Interferenzbilder mit einer festen Phasenverschiebung erstellt:

$$
\begin{aligned}
& I_{1}(x, y)=I_{0}\left[1+m(x, y) \cos \left(\Delta \varphi(x, y)+\Theta_{1}\right)\right] \\
& I_{2}(x, y)=I_{0}\left[1+m(x, y) \cos \left(\Delta \varphi(x, y)+\Theta_{2}\right)\right] \\
& I_{3}(x, y)=I_{0}\left[1+m(x, y) \cos \left(\Delta \varphi(x, y)+\Theta_{3}\right)\right]
\end{aligned}
$$

Das obige Gleichungssystem ist nach $\varphi(x, y)$ auflösbar. Die Vorteile bei Benutzung dieser Methode liegen nicht nur in der eindeutigen Bestimmbarkeit der gesuchten Phasenverschiebung, sondern auch darin, dass die sich ergebenden Phasenverschiebungsbilder weitestgehend unabhängig vom Phasenkontrast $m(x, y)$ und der Hintergrundintensität $I_{0}$ werden, da diese ebenfalls bestimmbar sind und aus den aufgenommenen Interferenzbildern herausgerechnet werden können. 

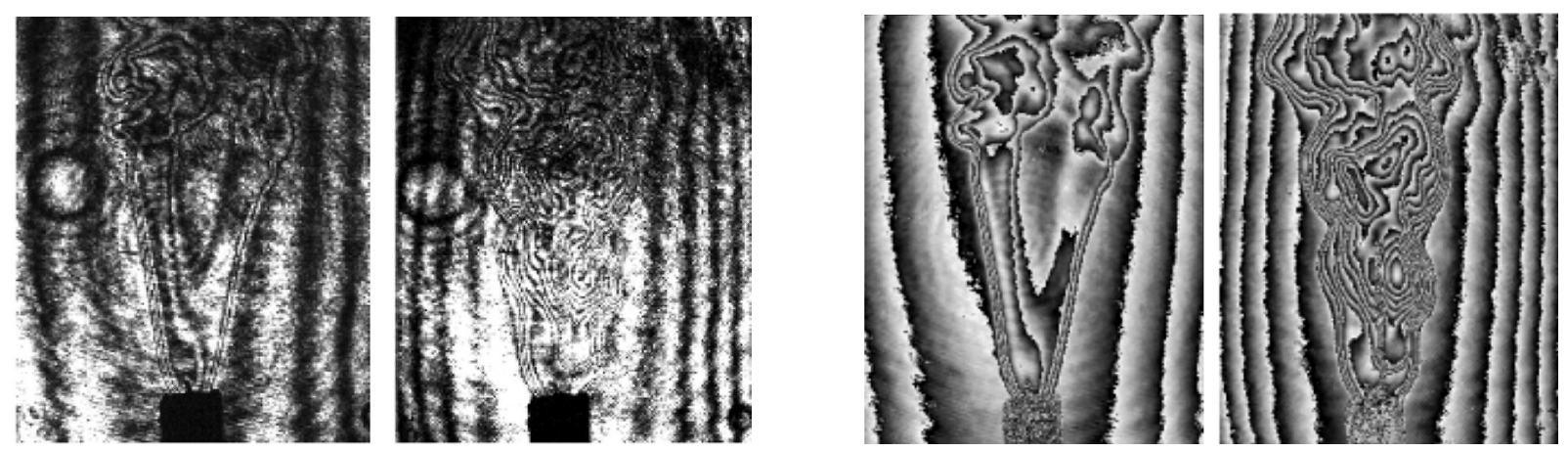

Abbildung 4.8: Beispiel für das Phasenschrittverfahren bei der holographischen Rekonstruktion: Die rekonstruierte Streifenverteilung (links) wird durch Bearbeitung mit dem Phasenschrittverfahren in eine vom Hintergrund und Phasenkontrast unabhängige Phasenverteilung (rechts) gewandelt.

Abb. 4.8 zeigt auf der linken Seite die Streifenverteilung von zwei Aufnahmen einer Kerze. Die ausgewerteten Bilder der Phasenverteilung mittels des Phasenschrittverfahrens sind in Abb. 4.8 rechts dargestellt. Hier zeigt sich sehr deutlich, wie die Phasenverteilung nicht mehr von der Hintergrundintensität beeinflusst wird; alle Störungen verschwinden. Die so erhaltene Phasenverschiebung erlaubt dann nach Gleichung 4.2, die hier noch einmal wiedergegeben wird, eine Bestimmung der Dichteverteilung.

$$
\Delta \phi(x, y)=\frac{1}{2 \pi \lambda} \int_{z_{1}}^{z_{2}}\left(\rho_{\infty} K_{\infty}-\rho(x, y, z) \sum_{i=1}^{N S} K_{i} \zeta_{i}(x, y, z)\right) d z
$$

Aus der Gleichung wird deutlich, dass für eine quantitative Bestimmung der Dichte eine Referenzdichte, hier die Dichte der freien Anströmung, bekannt sein muss. Darüberhinaus ist eine Kenntnis der Zusammensetzung des Gases oder genauer der Partialdichten $\zeta_{i}(x, y, z)$ im Raum nötig. In den Stoßschichten, die typischerweise im Versuch am HEG auftreten, ist dies in der Praxis kaum möglich, da durch die auftretenden chemischen Reaktionen der Gaszustand unbekannt ist. Darüber hinaus muss die Strömung axialsymmetrischen Charakter aufweisen oder echt zweidimensional sein, damit das obige Integral bestimmbar ist. Daher werden in dieser Arbeit aus gemessenen Phasenverschiebungen keine Dichteverteilungen zurückgerechnet, sondern aus den mit den numerischen Verfahren berechnenten Dichteverteilungen werden numerische Phasenverschiebungen erzeugt, die dann mit den experimentell gemessenen Phasenverschiebungen verglichen werden. Die numerischen Phasenverschiebungen entstehen mittels einer Strahlverfolgung, die die durch die Dichteunterschiede hervorgerufene Phasenverschiebung durch das dreidimensionale numerische Lösungsfeld aufintegriert. Hierzu wird die Gleichung 4.10 benutzt. 


\subsection{Messung von Oberflächendruck und Wandwärmestromdichte}

\subsubsection{Signalaufbereitung und Datenaufnahme}

An den in den Modellen verwendeten Sensoren für Druck und Temperatur werden typischerweise Spannungen im Millivoltbereich abgegriffen. Um diese Spannungen für die Messdatenaufnahme aufzubereiten, werden die Sensorsignale vor der Aufzeichnung durch einen mehrkanaligen Verstärker ${ }^{8}$ geführt. Dieser besitzt einen Frequenzbereich bis $200 \mathrm{kHz}$ bei -3db. Die maximale Verstärkung beträgt 1000. Dieser Verstärker wurde speziell für die Messungen mit piezoresistiven Sensoren und schnellen Thermoelementen entwickelt, die ausschließlich in den in der Messstrecke verwendeten Modellen und in der Sensorik zur Messung der freien Anströmung (siehe Abschnitt 3.3.5)) zum Einsatz kommen. Er versorgt die DMS-Brücken der Sensoren mit der erforderlichen Speisespannung $(2.5,5,7.5$ VDC) und bietet die Möglichkeit, diese vor Versuchsdurchführung abzugleichen.

Alle an der Anlage und an den Modellen vorhandenen Signale werden mit einem Transientenrekorder ${ }^{9}$ aufgezeichnet. Insgesamt stehen 86 Kanäle zur Verfügung. Alle Kanäle werden im Single-ended-Modus betrieben. Die Signale werden mit einem A/D-Wandler mit einer Auflösung von 12 Bit digitalisiert und gespeichert. Die Datenrate beträgt $1 \mathrm{MSam}-$ ple/s in einem Messzeitfenster von +/- 30 ms um den Triggerzeitpunkt und 0.1 MSample/s in einem Messzeitfenster von +/- 500 ms um den Triggerzeitpunkt. Als Triggersignal für den Transientenrekorder dient das Stoßgeschwindigkeitssignal (s. Abschnitt 3.3.2) und aus Sicherheitsgründen zusätzlich das Drucksignal am Stoßrohrende (s. Abschnitt 3.3.4).

Die gemessenen Signale werden vor der weiteren digitalen numerischen Verarbeitung gefiltert, um Rauschen und Störsignale zu entfernen. Hierzu wird ein Filter von Savietzky und Golay verwandt (Press u. a. (1992)). Die Ordnung des Filters beträgt 6 und die Glättung läuft über 64 Punkte. Ohne die Anwendung dieses Filters wird vor allem die Auswertung der Oberflächentemperaturdaten in Wärmestromdichten problematisch, da Störungen auf den Signalen bei der Auswertung extrem verstärkt werden.

\subsubsection{Oberflächendruck}

Die in dieser Arbeit verwendeten Druckaufnehmer werden von der Firma KULITE ${ }^{10}$ hergestellt. Bei diesen piezoresistiven Druckaufnehmern wird durch eine Druckwirkung der Widerstand im Material verändert, sie sind deshalb für statische sowie für dynamische Anwendungen geeignet. Sie sind passiv, benötigen also eine Speisung. Die Widerstandsänderung resultiert aus zwei überlagerten Effekten. Zum einen bewirkt eine Dehnung des Messkörpers eine Querschnittsverringerung und dadurch eine Erhöhung des Widerstandes.

\footnotetext{
${ }^{8}$ Modell HERO ${ }^{R}$ DMS9303C-LT-200 der Firma Rohrer GmbH Mess-\& Systemtechnik, Postfach 752 , 81457 München. [www.rohrer-muenchen.de]

${ }^{9}$ Modell TRC 6510 der Firma Eckelmann AG, Berliner Straße 161, 65205 Wiesbaden, [www.eckelmann.de]

${ }^{10}$ KULITE Semi-Conductor GmbH, Postfach 1527, 65705 Hofheim/Ts, [www.kulite.de]
} 


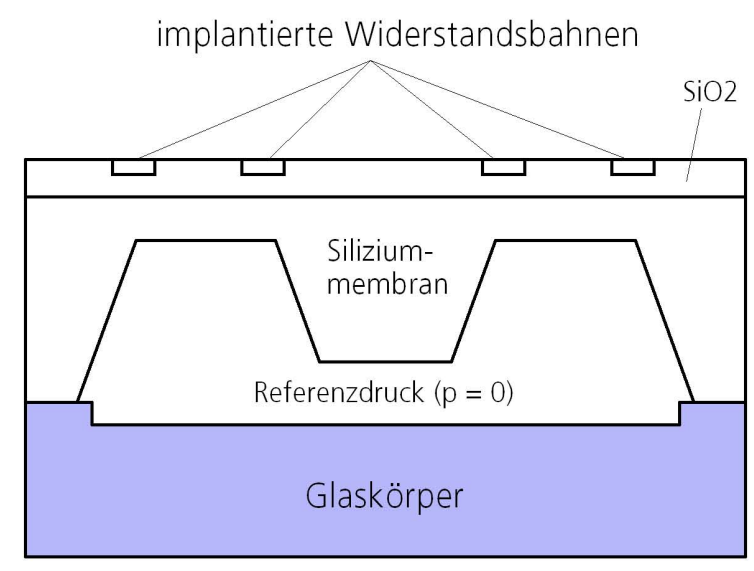

unbelastet

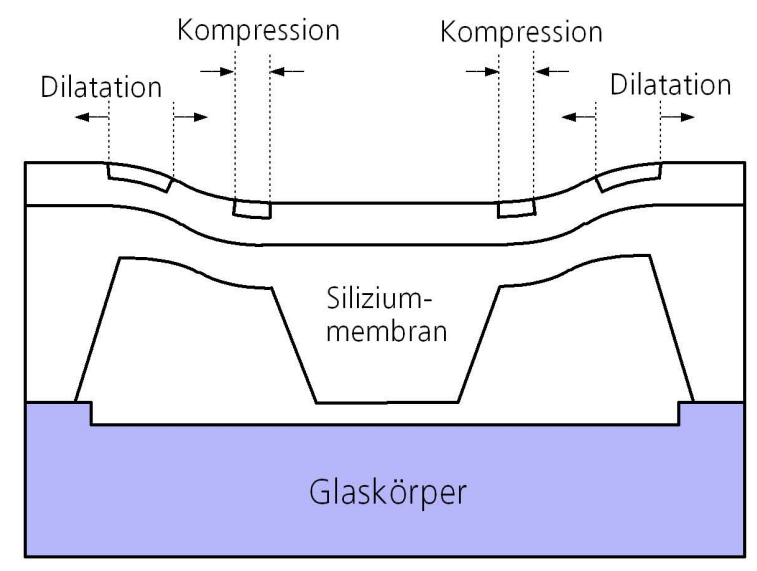

belastet

Abbildung 4.9: Schematische Darstellung des Funktionsprinzips eines piezoresistiven Druckaufnehmers der Firma Kulite (KULITE (2004)).

Zum anderen verändert sich der spezifische Widerstand mit der Dehnung. Dieser zweite Effekt wird piezoresistiver Effekt genannt. Bei Halbleitermaterialien ist der piezoresistive Effekt sehr viel ausgeprägter als bei Metallen. Er hängt ab von der Orientierung des Halbleiter-Einkristalles und von der Dotierung (Art, Dichte und Verteilung der Fremdatome, welche die Leitfähigkeit bestimmen). Bei Halbleiter-DMS ist der piezoresistive Effekt etwa 50 mal stärker als bei metallischen DMS. Obwohl der piezoresistive Effekt nicht allein dieser Gruppe vorbehalten ist, hat sich die Bezeichnung piezoresistiver Sensor für die Aufnehmer durchgesetzt, bei denen die sich unter Druck deformierende Struktur in einem Siliziumträger integriert ist. Durch die Miniaturisierung dieses Trägers und die direkte Verwendung als Messmembran sind diese Aufnehmer gut für dynamische Messungen geeignet, da sie eine hohe Eigenfrequenz von mehreren hundert $\mathrm{kHz}$ besitzen. Bei den Sensoren der Firma KULITE sind in die Oberfläche des Siliziumträgers vier Widerstandsbahnen implantiert (KULITE (2004)). Durch eine entsprechende Ausrichtung dieser Bahnen werden bei Verformung der Membran durch Druckeinwirkung je zwei Widerstandsbahnen gestaucht und zwei Widerstandsbahnen gedehnt. In Abb. $\mathbf{4 . 9}$ ist die Membran im Schnitt schematisch im unbelasteten und belasteten Zustand dargestellt.

Die Widerstandsbahnen sind zu einer Vollbrücke verschaltet, an der das Messsignal abgegriffen wird. Die abgegriffene Spannung ist proportional zu dem auf die Membran einwirkenden Druck. In Tab. 4.3 sind die wichtigsten technischen Spezifikationen der in dieser Arbeit verwendeten Druckaufnehmer angegeben. Die Temperaturkompensation der abge-

\begin{tabular}{|ll|}
\hline Nichtlinearität & $0.15 \%$ vom Eingangsbereich $(0.3 \%$ maximal) \\
Hysterese & $0.1 \%$ vom Eingangsbereich \\
Reproduzierbarkeit & $0.1 \%$ vom Eingangsbereich \\
Eigenfrequenz & $150 \mathrm{kHz}$ \\
Thermische Nullpunktsdrift & $1 \%$ vom Eingangsbereich $/ 55^{\circ} \mathrm{C}$ \\
Thermische Empfindlichkeitsdrift & $1 \%$ vom Eingangsbereich $/ 55^{\circ} \mathrm{C}$ \\
\hline
\end{tabular}

Tabelle 4.3: Kenndaten für die verwendeten KULITE-Druckaufnehmer. 

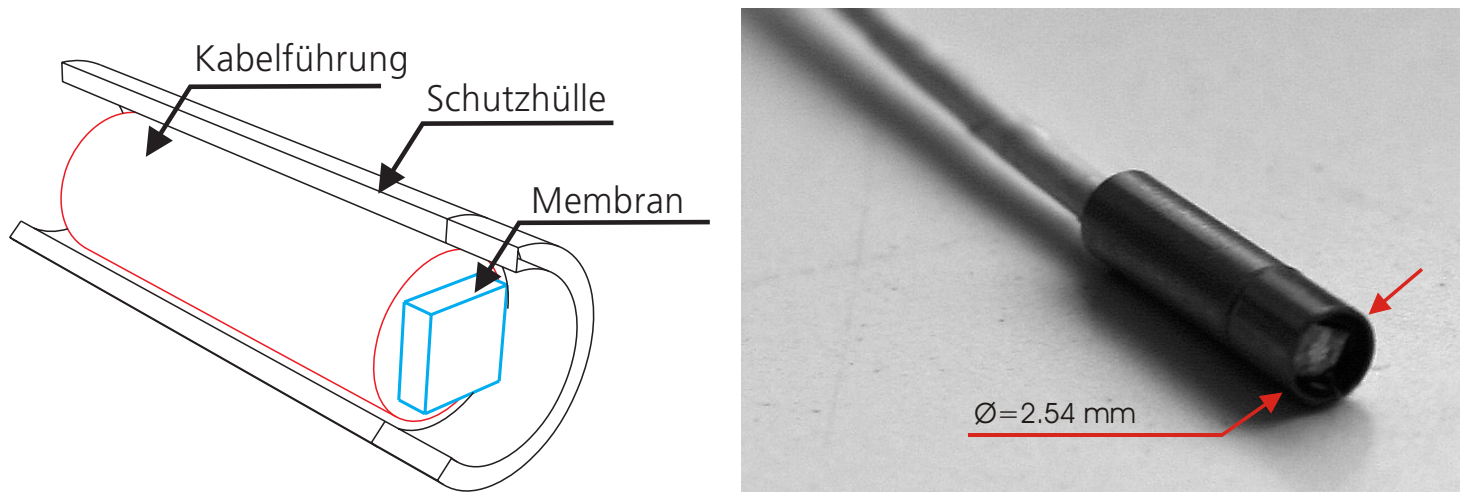

Abbildung 4.10: Aufbau der verwendeten piezoresistiven Drucksensoren der Firma KULITE.

griffenen Spannung erfolgt durch eine zusätzliche Verschaltung innerhalb des Sensorkabels. Diese Kompensation ist für die Versuchszeit im HEG ohne Funktion, da sich der Modellinnenraum während der kurzen Versuchszeit nicht erwärmt (s. Abschnitt 4.2.3). In Abb. 4.10 ist ein Druckaufnehmer des Typs XCS 093 im Schema und als Photographie dargestellt. Die Serienmodelle besitzen über der Siliziummembran ein Schutzgitter. Dieses Gitter wird für die am HEG verwendeten Sensoren vom Hersteller entfernt, da es im Laufe der Zeit durch die im HEG auftretenden Verunreinigungen zugesetzt wird und dann deutlichen Einfluss auf die Reaktionszeit des Sensors hat.

Das Zylindermodell, das schon bei früheren Untersuchungen von Stoß/Stoß-Wechselwirkungen eingesetzt wurde, besaß Wanddruckanbohrungen von $1 \mathrm{~mm}$ Durchmesser. Die damaligen Untersuchungen wiesen starke Temperatureinwirkungen auf die Sensoren auf, die zum Teil auch zur völligen Zerstörung der Sensorik führten. Zur Unterdrückung der direkten Tempe-
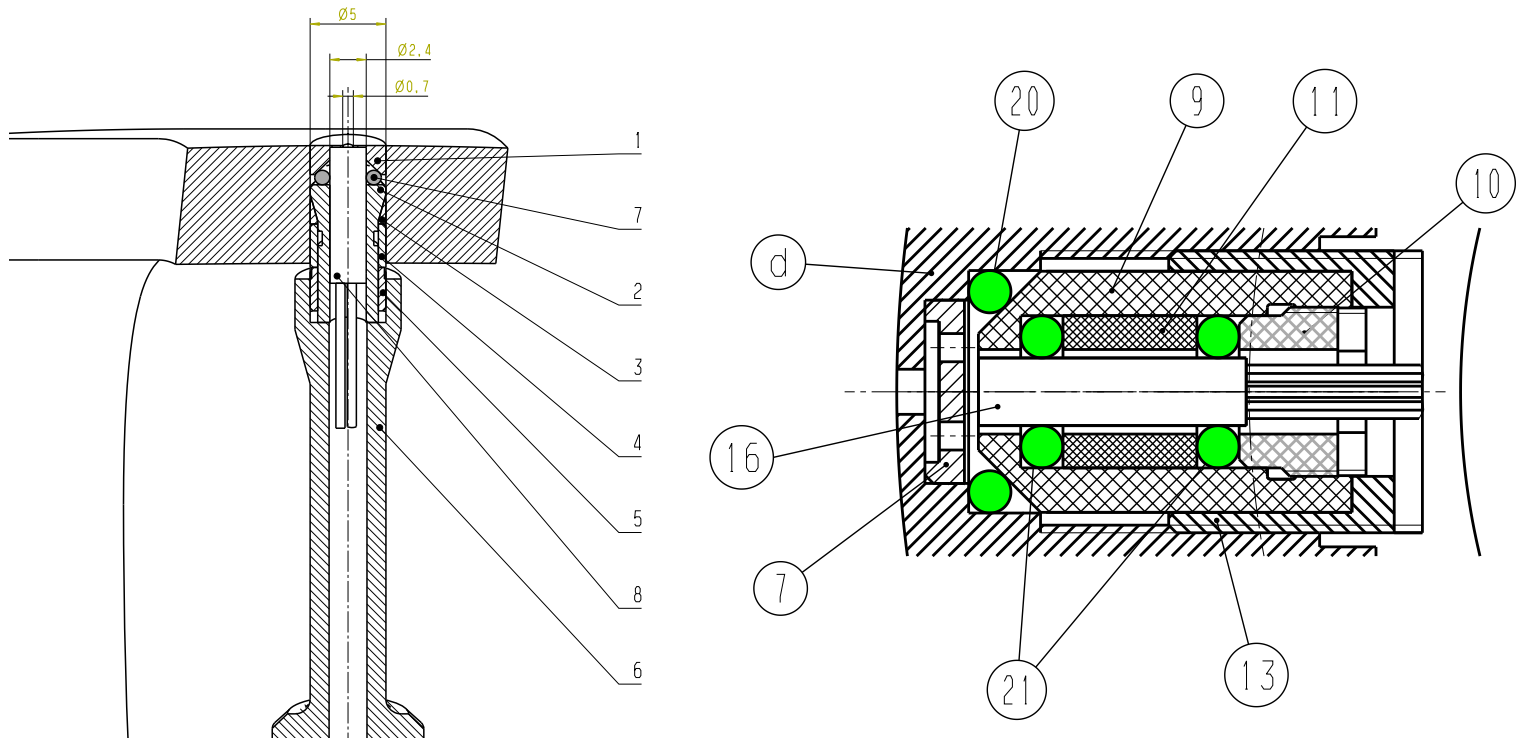

Abbildung 4.11: Verwendete Drucksensoreinbauten: (links) Einbau im Model des X-38 (Modellbeschreibung in Abschnitt 4.3.3). Der O-Ring(7) dient der Abdichtung des Sensors. (rechts) Einbau im Zylindermodell und Doppelrampenmodell (Modellbeschreibungen in Abschnitt 4.3.1 und Abschnitt 4.3.2). 


\begin{tabular}{|c|c|c|c|}
\hline Typ & Material A (+) & Material B (-) & Bereich \\
\hline$B$ & Platin 30\% Rhodium & Platin 6\% Rhodium & $1370^{\circ} \mathrm{C}-1700^{\circ} \mathrm{C}$ \\
\hline C & W5Re Wolfram 5\% Rhenium & W26Re Wolfram 26\% Rhenium & $1650^{\circ} \mathrm{C}-2315^{\circ} \mathrm{C}$ \\
\hline$E$ & Chromel & Konstantan & $95^{\circ} \mathrm{C}-900^{\circ} \mathrm{C}$ \\
\hline J & Eisen & Konstantan & $95^{\circ} \mathrm{C}-760^{\circ} \mathrm{C}$ \\
\hline K & Chromel & Alumel & $95^{\circ} \mathrm{C}-1260^{\circ} \mathrm{C}$ \\
\hline N & Nicrosil & Nisil & $650^{\circ} \mathrm{C}-1260^{\circ} \mathrm{C}$ \\
\hline R & Platin $13 \%$ Rhodium & Platin & $870^{\circ} \mathrm{C}-1450^{\circ} \mathrm{C}$ \\
\hline S & Platin $10 \%$ Rhodium & Platin & $980^{\circ} \mathrm{C}-1450^{\circ} \mathrm{C}$ \\
\hline $\mathrm{T}$ & Kupfer & Konstantan & $-200^{\circ} \mathrm{C}-350^{\circ} \mathrm{C}$ \\
\hline Chromel & \multirow{5}{*}{\multicolumn{3}{|c|}{$\begin{array}{l}\text { 90\% Nickel 10\% Chrom } \\
95 \% \text { Nickel 2\% Mangan 2\% Alumimnium 1\% Silizium } \\
55 \% \text { Kupfer } 45 \% \text { Nickel } \\
95.6 \% \text { Nickel } 4.4 \% \text { Silizium } \\
84.1 \% \text { Nickel } 1.3 \% \text { Silizium } 14.6 \% \text { Chrom }\end{array}$}} \\
\hline Alumel & & & \\
\hline Constantan & & & \\
\hline & & & \\
\hline Nicrosil & & & \\
\hline
\end{tabular}

Tabelle 4.4: ISO-Kodierung für die gebräuchlichsten Materialkombinationen für Thermoelemente.

ratureinwirkung wurden kleine Umlenkplatten (Abb. 4.11, Teil 7) vor die Sensoren eingesetzt, so dass die Sensormembranen geschützt platziert werden konnten. Bei den ersten Messungen im HEG mit diesen Einsätzen erwies sich die Druckanstiegszeit aber als zu lang, und als Lösung wurden die Druckanbohrungen auf $1.5 \mathrm{~mm}$ aufgeweitet (Schnieder (1999)). So ausgerüstet wurde das Modell in dieser Arbeit benutzt. Die Bauteile (9-11) in Abb. 4.11 bilden zusammen mit den O-Ringen (21) einen Einsatz, in den der Drucksensor (16) eingebaut wird. Der gesamte Einsatz mit Drucksensor wird mit Hilfe einer Gewindebuchse (13) und einem O-Ring (20) druckdicht in der Modellwand (d) verschraubt. Die ersten Experimente mit dem Doppelrampenmodell, für das ein sehr kleiner Durckanbohrungsdurchmesser $(0.8 \mathrm{~mm})$ gewählt wurde, zeigten diese Problematik nicht, so dass auf die Umlenkplatten verzichtet werden konnte. Die Abb. 4.11b zeigt, wie die Sensoren im Zylindermodell und im Doppelrampenmodell in der Wand platziert werden. Für das Modell des X-38 wurde eine neue Möglichkeit des Einbaus entwickelt. Der Modellwandeinsatz mit Druckanbohrung wird nach der Fertigung verklebt und der Oberfläche angepasst. Der Drucksensor (8) wird in der Sensorhalterung (2-5) eingesetzt, und der gesamte Aufbau wird mit dem Einbauwerkzeug (6) in der Modellwand mit Hilfe kleiner Schnellspanneinrichtungen in der Modellwand fixiert. Die Dichtung erfolgt auch hier über einen O-Ring am Sensorkopf. Der Durchmesser der Wanddruckanbohrungen wurde auf $0.7 \mathrm{~mm}$ reduziert.

\subsubsection{Oberflächenwärmestromdichte}

Für die Messung des zeitlichen Verlaufes der Oberflächentemperatur kommen miniaturisierte Thermoelemente koaxialer Bauform zum Einsatz. Diese Thermoelemente werden von der Firma MedTherm ${ }^{11}$ in Handarbeit gefertigt und sind in unterschiedlichen Durchmessern erhältlich $(0.38 \mathrm{~mm}, 0.38 \mathrm{~mm}, 0.78 \mathrm{~mm}, 1.55 \mathrm{~mm}, 2.51 \mathrm{~mm})$. Die bei den experimentellen Untersuchungen in dieser Arbeit verwendeten Elemente sind vom ISA ${ }^{12}$ Typ $E^{13}$. In Tab. 4.4 befindet sich eine Auflistung der gebräuchlichsten Materialkombinationen.

\footnotetext{
${ }^{11}$ MedTherm Corporation, POST OFFICE BOX 412 / HUNTSVILLE, ALABAMA 35804.

${ }^{12}$ Instrument Society of America.

${ }^{13}$ Diese Thermoelemente bestehen aus den Materialien Chromel ( $\mathrm{Ni}-\mathrm{Cr}$ ) und Konstantan (Cu-Ni).
} 

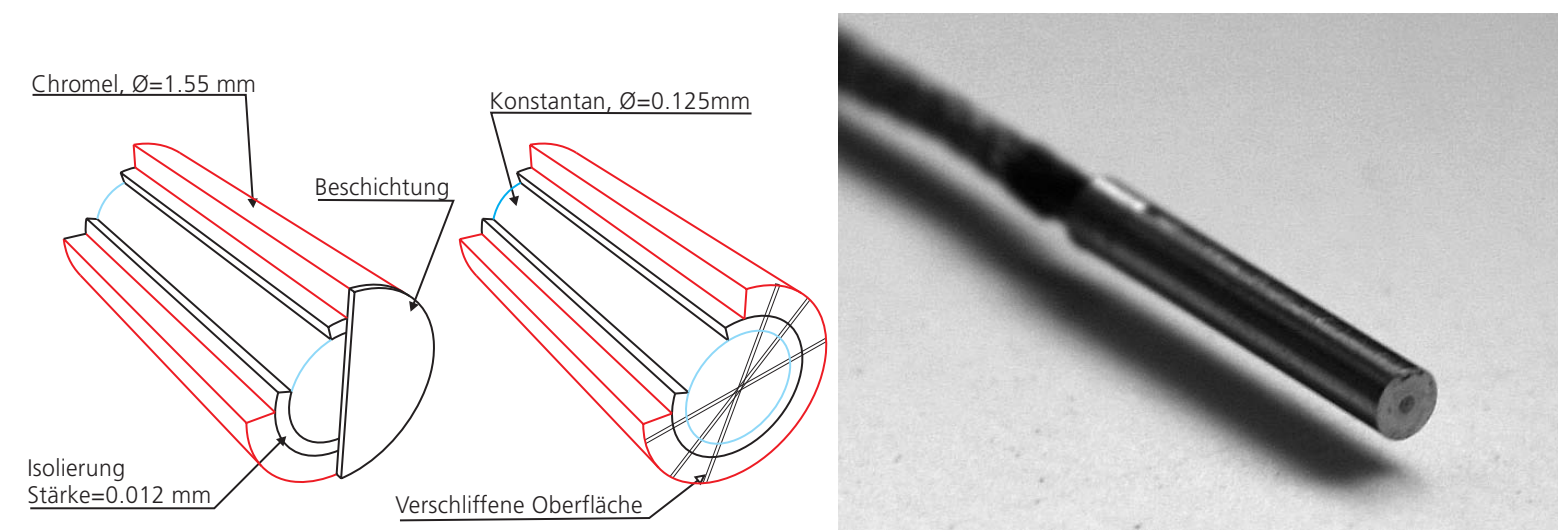

Abbildung 4.12: Miniaturisiertes Thermoelement der Firma MedTherm: (links) Schematische Darstellung, (rechts) Photographie des Typs TCS-061-E-0.9-24-10866.

In Abb. 4.12 ist ein Thermoelement des MedTherm-Typs TCS-061-E-0.9-24-10866 schematisch dargestellt. Den Kern bildet ein Konstantan-Thermodraht mit $0.125 \mathrm{~mm}$ Durchmesser. Eine Isolierschicht mit $0.012 \mathrm{~mm}$ Dicke trennt diesen Konstantandraht von der ihn umgebenden Chromel-Hülle. Im Auslieferungszustand sind die Thermoelemente mit einer elektrisch leitenden Schicht bedampft. Diese Schicht bildet eine sehr kleine Kontaktmasse und bedingt die schnelle Ansprechzeit, die vom Hersteller mit $1 \mu$ s angegeben ist. Bei der Verwendung in Modellen wird diese Schicht normalerweise durch das Anpassen der Thermoelemente an die Oberfläche mittels Schleifen entfernt.

Durch manuelles Ritzen oder Schleifen über die beiden Thermopartner wird dieser Kontakt durch die sich über die Isolationsschicht hinweg bildenden Metallspäne vor dem Experiment wieder hergestellt. Es ist also nicht nur möglich, das Thermoelement mechanisch zu bearbeiten und der gegebenen Körperkontur anzupassen, sondern es bildet sich dabei sogar unmittelbar die Messstelle. In Abb. 4.12 ist dies schematisch darstellt. Bei der Auswertung wird das Thermoelementsignal, wie auch bei den Drucksensorsignalen, zuerst auf den Stoßreflektionszeitpunkt normiert, gefiltert und dann der Offset entfernt. Die Umrechnung der gemessenen Thermospannungen in Temperatur erfolgt mittels eines Polynoms:

$$
\Delta T=17.02252 \Delta U-0.2209724 \Delta U^{2}+5.481 \cdot 10^{-3} \Delta U^{3}-5.767 \cdot 10^{-5} \Delta U^{4},
$$

wobei die Spannung in $\mathrm{mV}$ eingesetzt wird. Für die Bestimmung der Wärmestromdichte müssen Annahmen getroffen werden. Da die Eindringtiefen innerhalb der kurzen Messzeiten im HEG klein sind, können zwei das Problem wesentlich vereinfachende Annahmen getroffen werden. Der Sondenkörper wird erstens als halbunendlich ausgedehnt betrachtet, und zweitens wird eine ausschließlich eindimensionale Wärmeleitung in den Sondenkörper hinein angenommen. Die erste Annahme ist gültig, solange sich das im Modell befindliche Ende des Thermoelements nicht erwärmt. Sie lässt sich auch im Experiment im HEG durch eine Temperaturmessung innerhalb eines Windkanalmodells überprüfen und bestätigen (s. Kastell (1997)). Schultz u. Jones (1973) geben als Faustformel für die Materialtiefe, in der Temperatur und Wärmestromdichte 1\% des Oberflächenwertes erreicht haben, 


\begin{tabular}{|l|c|c|c|c|c|c|}
\hline Größe & Symbol & Einheit & Nickelchrom & Konstantan & Edelstahl & Kalibrierung \\
\hline Dichte & $\rho$ & $\mathrm{kg} / \mathrm{m}^{3}$ & 8719.17 & 8912.90 & 8054.85 \\
spezifische Wärmekapazität & $\mathrm{c}$ & $\mathrm{J} /(\mathrm{kg} \mathrm{K})$ & 448.00 & 393.60 & 2.40 \\
Wärmeleitfähigkeit & $\mathrm{k}$ & $\mathrm{W} /(\mathrm{m} \mathrm{K})$ & 18.09 & 19.96 & 16.22 \\
Temperaturleitzahl & $\mathrm{a}$ & $10^{-6} \mathrm{~m}^{2} / \mathrm{s}$ & 4.63 & 5.69 & 4.01 \\
$(\rho \mathrm{ck})^{0.5}$ & $\mathrm{~b}$ & $\mathrm{~J} /\left(\mathrm{m}^{2} \mathrm{~K} \mathrm{~s}^{1 / 2}\right)$ & 8406.12 & 8367.90 & 8102.90 \\
\hline
\end{tabular}

Tabelle 4.5: Stoffwerte der verwendeten Thermoelemente für die Bestimmung der Wärmestromdichte. Die Werte sind von Kidd u. a. (1994) publiziert worden und beziehen sich auf T=300 K.

$$
x=4 \sqrt{a t}
$$

an, wobei t die Einwirkzeit und $a$ die Temperaturleitfähigkeit ist. Für eine typische Länge eines in den Modellen benutzten Thermoelementes von $5 \mathrm{~mm}$ ergibt sich eine Messdauer von 0.3 s. Diese Zeit ist zwei Größenordnungen größer als die Messzeit im HEG. Für die Annahme der Eindimensionalität muss sichergestellt werden, dass nur auf der der Strömung zugewandten Fläche des Sensors Wärme eindringen kann; es darf keine laterale Leitung unter der Oberfläche innerhalb der Messzeit stattfinden. Gelten diese Annahmen, dann wird die eindimensionale Wärmeleitung in den halbunendlichen Körper durch die folgende Differentialgleichung beschrieben:

$$
\frac{\partial T}{\partial t}=a \frac{\partial^{2} T}{\partial x^{2}}+\frac{\dot{q}_{i}}{\rho c} .
$$

T ist die Temperatur, $x$ gibt die Koordinatenrichtung in den Körper hinein an, $\mathrm{t}$ ist die Zeit, $\dot{q}_{i}$ sind Wärmequellen und a ist die Temperaturleitfähigkeit oder Temperaturleitzahl. Sie ist definiert durch das Verhältnis von Wärmeleitfähigkeit $k$ zu dem Produkt aus Dichte $\rho$ und spezifischer Wärmekapazität $c$ :

$$
a=\frac{k}{\rho c} .
$$

In Tab. 4.5 sind die Werte für das verwendete Thermoelementmaterial und den Edelstahl, aus dem die Modelle gefertigt sind, aufgeführt. Da streng genommen alle Werte von der Temperatur abhängen, ist eine theoretische Bestimmung schwierig, darüber hinaus ist nicht bekannt, wie der Wert, der sich im Thermoelement aus einer Kombination der Werte beider Thermomaterialien zusammensetzen muss, zu bestimmen ist. Der in dieser Arbeit benutzte Wert enstammt nicht der Literatur, sondern basiert auf einer vom Auto durchgeführten experimentellen Kalibrierung der MedTherm-Thermoelemente (s. Anhang A).

Sind keine Wärmequellen $\dot{q}_{i}$ im Problem vorhanden, so reduziert sich Gleichung $4.11 \mathrm{zu}$

$$
\frac{\partial^{2} T}{\partial x^{2}}=\frac{1}{a} \frac{\partial T}{\partial t} .
$$

Diese Gleichung beschreibt die zeitabhängige Temperaturverteilung im Sondenkörper. Mit den Randbedingungen $\dot{q}_{S}(x=0)=-k \partial T / \partial x, T(x=\infty)=0$ erhalten Schultz u. Jones (1973) in ihrem Übersichtsbericht für die Wärmestromdichte: 


$$
\dot{q}_{S}(t)=\sqrt{\frac{\rho c \kappa}{\pi}}\left[\frac{T(t)}{\tau}+\frac{1}{2} \int_{0}^{t} \frac{T(t)-T(\tau)}{(t-\tau)^{\frac{3}{2}}} d \tau\right] .
$$

Mit Hilfe dieser Bestimmungsgleichung ist es möglich, wenn die obigen Annahmen erfüllt sind, aus einem gemessenen Oberflächentemperaturverlauf die Wärmestromdichte in der Oberfläche zu bestimmen. Die Schreibweise in Gleichung 4.12 ist für die Auswertung der in digitaler Form vorliegenden Messdaten jedoch ungünstig und wird durch eine Diskretisierung ersetzt. In dieser Arbeit wird die Methode von Cook u. Feldermann (1966) benutzt, die die folgende Diskretisierung vorschlagen:

$$
\dot{q}_{S}\left(t_{n}\right)=2 \sqrt{\frac{\rho c \kappa}{\pi}} \sum_{j=1}^{n} \frac{T_{j}-T_{j-1}}{\sqrt{t_{n}-t_{j}}+\sqrt{t_{n}-t_{j-1}}} .
$$

Unter der Annahme einer konstanten Wärmestromdichte an der Wand $\left(q_{S}(t)=\right.$ konst. $)$ lässt sich die Gleichung 4.2.3 exakt zu

$$
T_{W}(x=0, t)=\frac{2 \dot{q}_{S}}{\sqrt{\pi}} \sqrt{\frac{t}{\rho \cdot c \cdot k}}
$$

Iösen. Der Temperaturverlauf ist dann nur noch eine Funktion der Zeit $t$, der konstanten Wärmestromdichte an der Wand $\dot{q}_{S}$ und der Materialdaten des Sensors.

In Abb. 4.13 ist eine Auswertung der Wärmestromdichte auf der permanenten Sonde für die HEG-Bedingung I dargestellt. Die Thermoelemente werden vor dem Versuch auf Null normiert, da das Polynom (s. Gleichung 4.2.3), das zur Berechnung der Temperatur herangezogen wird, auf Raumtemperatur bezogen ist. In Abb. 4.13 auf der linken Seite ist der gemessene Temperaturverlauf im Staupunkt auf einer Halbkugel dargestellt, auf der rechten Seite die nach Gleichung 4.13 bestimmte Wärmestromdichte. Die schwarze Kurve
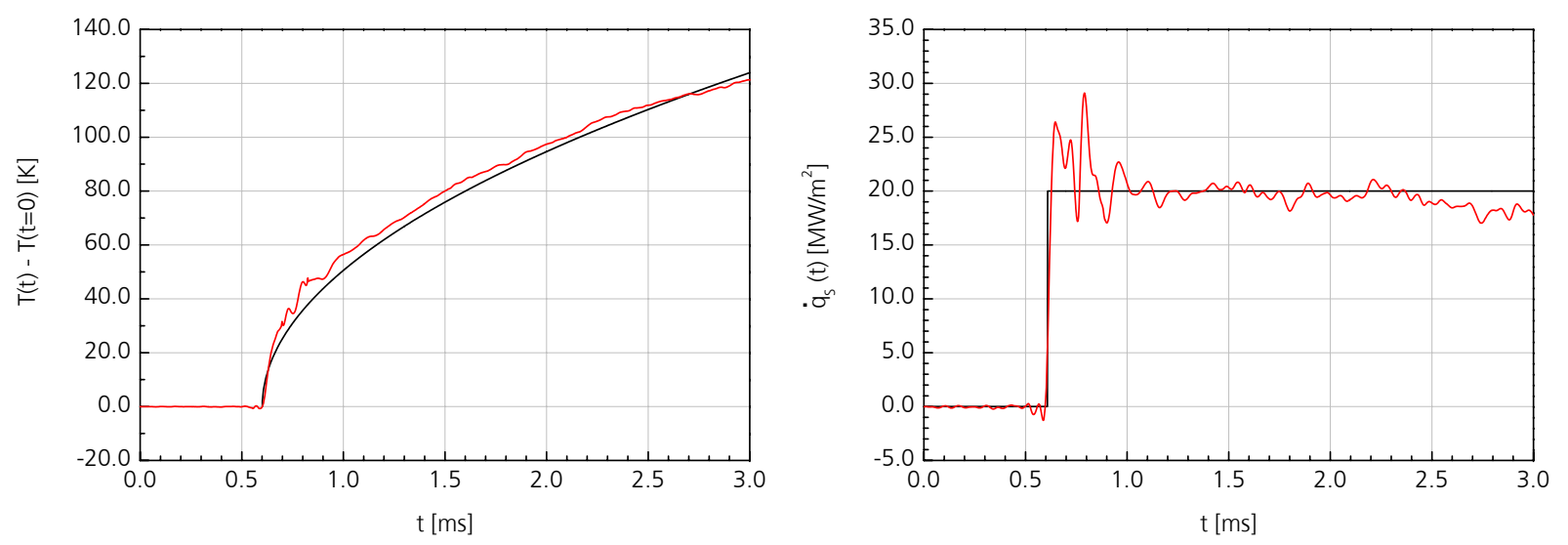

Abbildung 4.13: Gemessener Temperaturverlauf (links) im Staupunkt einer Halbkugel (Versuch 619, siehe auch Abschnitt 3.3.5) und die daraus bestimmte Wärmestromdichte (rechts), dargestellt in Rot. 
in Abb. 4.13 zeigt den sich ergebenden theoretischen Temperaturanstieg bei Annahme einer konstanten Wärmestromdichte an dem Sensor, wie in Abb. 4.13 auf der rechten Seite dargestellt.

\subsection{Verwendete Modelle}

\subsubsection{Zylindermodell}

Das für die Messung der Zylinderumströmung verwendete Modell hat einen Radius von 45 mm und eine Länge von 380 mm. Das Modell ist aus Edelstahl (1.0570, ST52-3, EN 10025) gefertigt. Das Modell wurde auf der Achse der Düse quer zur Anströmung ausgerichtet. Eine Photographie des Zylindermodells in der Messstrecke ist in Abb. $\mathbf{4 . 1 4}$ gegeben. Das für die Diskussion der Messergebnisse verwendete Koordinatensystem ist in Abb. 4.14 auf der rechten Seite dargestellt. Das Modell ist mit 17 Drucksensoren und 17 Thermoelementen ausgerüstet. Die Einbauart für die Drucksensoren ist in Abschnitt 4.2.2 und für die Thermoelemente in Abschnitt 4.2.3 beschrieben. Da nicht genug Platz im Modell vorhanden ist, sind die Sensoren auf 5 Ebenen in Spannweitenrichtung verteilt (s. Abb. 4.15). Hierdurch ist eine Winkelauflösung sowohl für die Thermoelemente als auch für die Drucksensoren von $5^{\circ}$ realisiert worden. Die Messebenen haben in Spannweitenrichtung einen Abstand von $10 \mathrm{~mm}$. Rechts und links in der Vorderansicht des Zylindermodells in Abb. 4.15 sind der Vollständigkeit halber auch die im DLR entwickelten Thermoelemente, die im Modell verklebt wurden (siehe Schnieder (1999)), mit eingetragen.

Diese Thermoelemente wurde vom Verfasser geeicht und kalibriert, aber aufgrund des schlechten Signal-Rausch-Verhältnisses und der ungenügenden Reproduzierbarkeit während der Versuche bei der Auswertung nicht berücksichtigt. Während der ersten Versuche
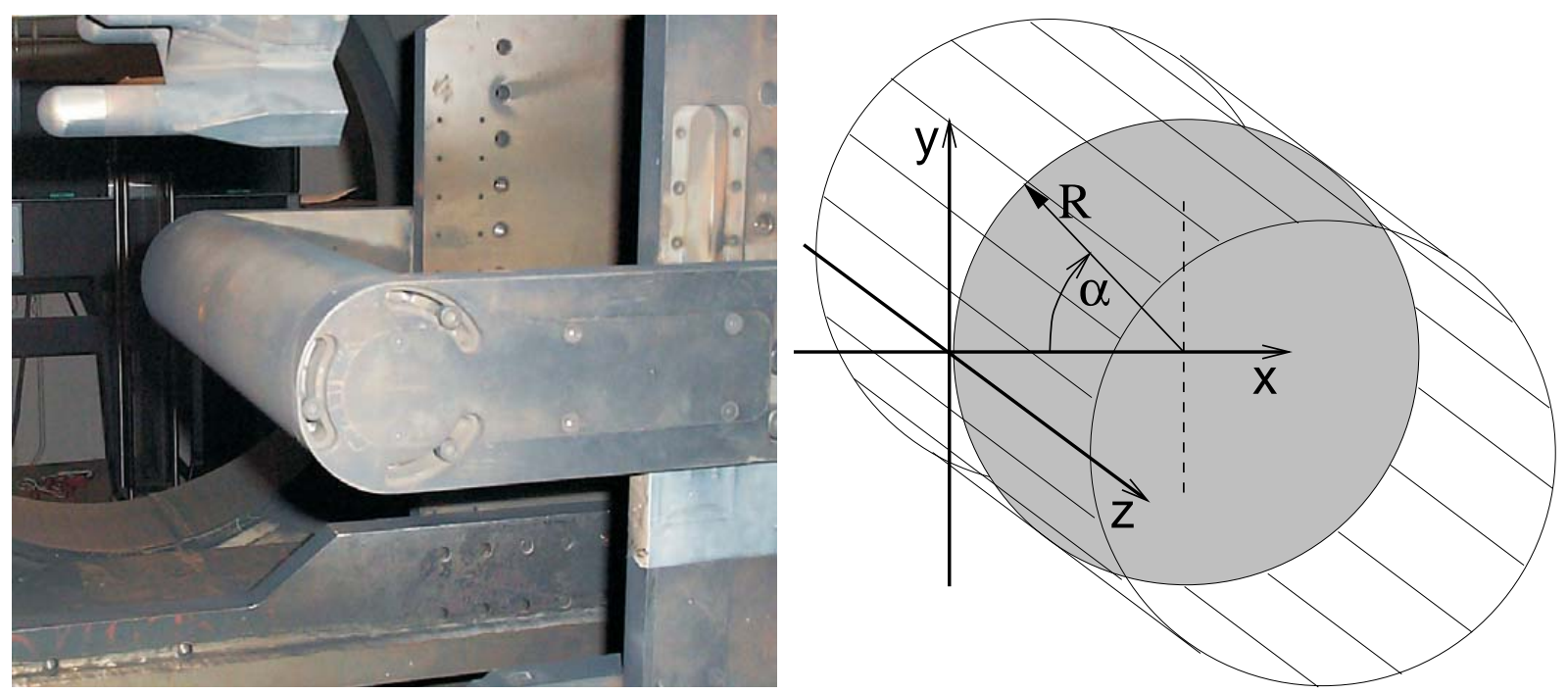

Abbildung 4.14: Photographie des eingebauten Zylindermodells (links) in der HEG-Messstrecke. Der Düsenaustritt befindet sich auf der linken Seite des Bildes. Oberhalb des Modells befindet sich die permanente Sonde. Rechts das Koordinatensystem für das Zylindermodell. 

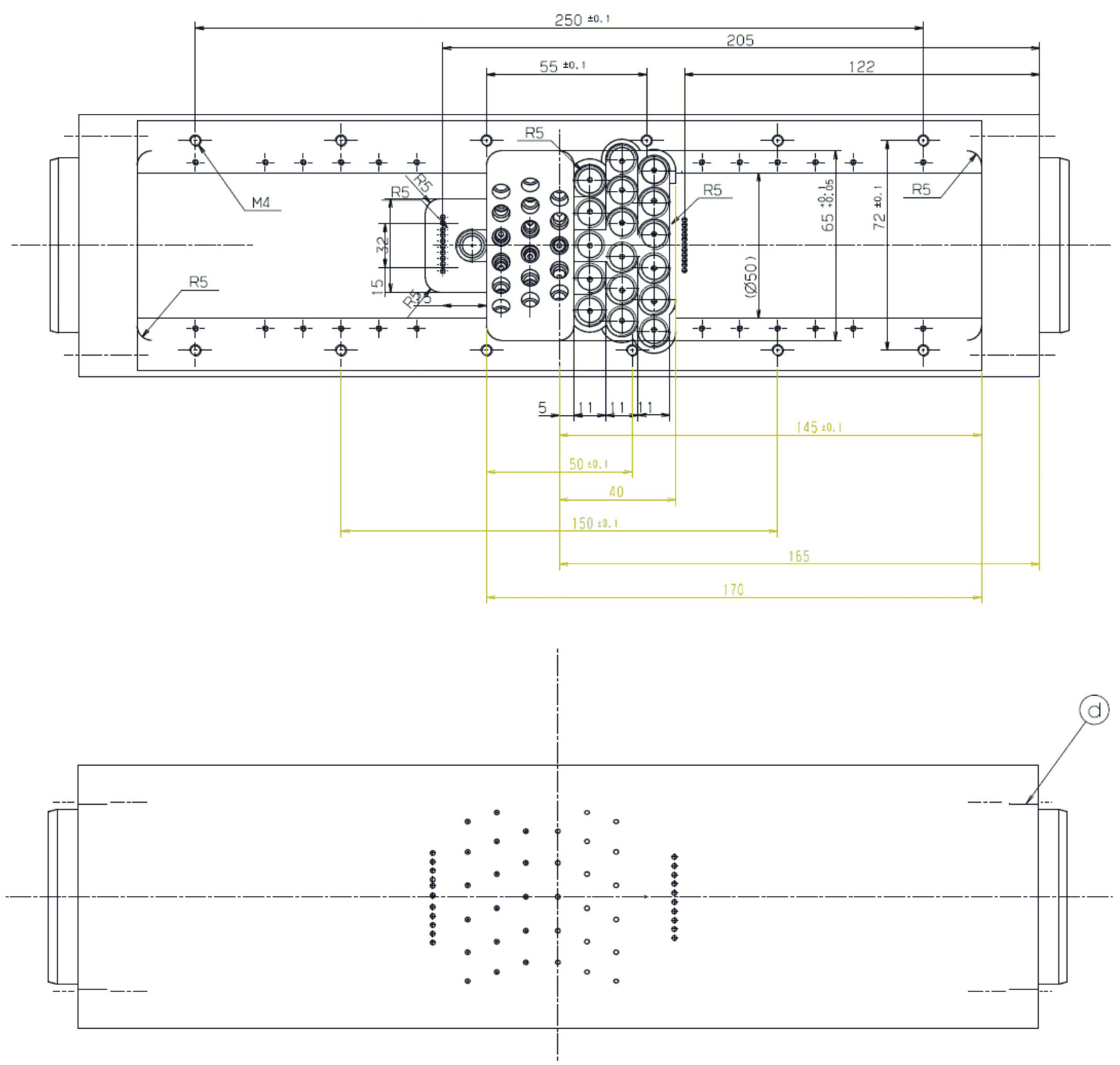

Abbildung 4.15: Innenansicht des Zylindermodells (oben) und Ansicht von vorn (unten). Die mit (d) beschrifteten Markierungen sind Justiermarken auf der Zylinderoberfläche, um den Anstellwinkel des Modelles einzustellen.

wurde deutlich, dass die Oberfläche des Zylindermodells, das schon für Messungen über Stoß-Stoß-Wechselwirkungen benutzt wurde (Schnieder (1999)), so große Unebenheiten aufwies, dass die Wärmestrommessungen gestört wurden. Wiederholversuche unter verschiedenen Anstellwinkeln zeigten, dass diese Störungen mit Änderung der Position der Beschädigungen auch die Position veränderten. Da keine Möglichkeit bestand, einen neuen Modellkörper zu fertigen, wurde die Oberfläche durch das Auffüllen der beschädigten Stellen mit Spritzspachtel ${ }^{14}$ ausgeglichen und mit feinem Schleifpapier nass verschliffen. Durch das saubere Überarbeiten der Oberfläche vor jedem Versuch ließen sich die Störungen weitestgehend unterdrücken.

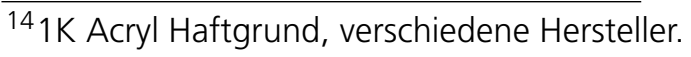



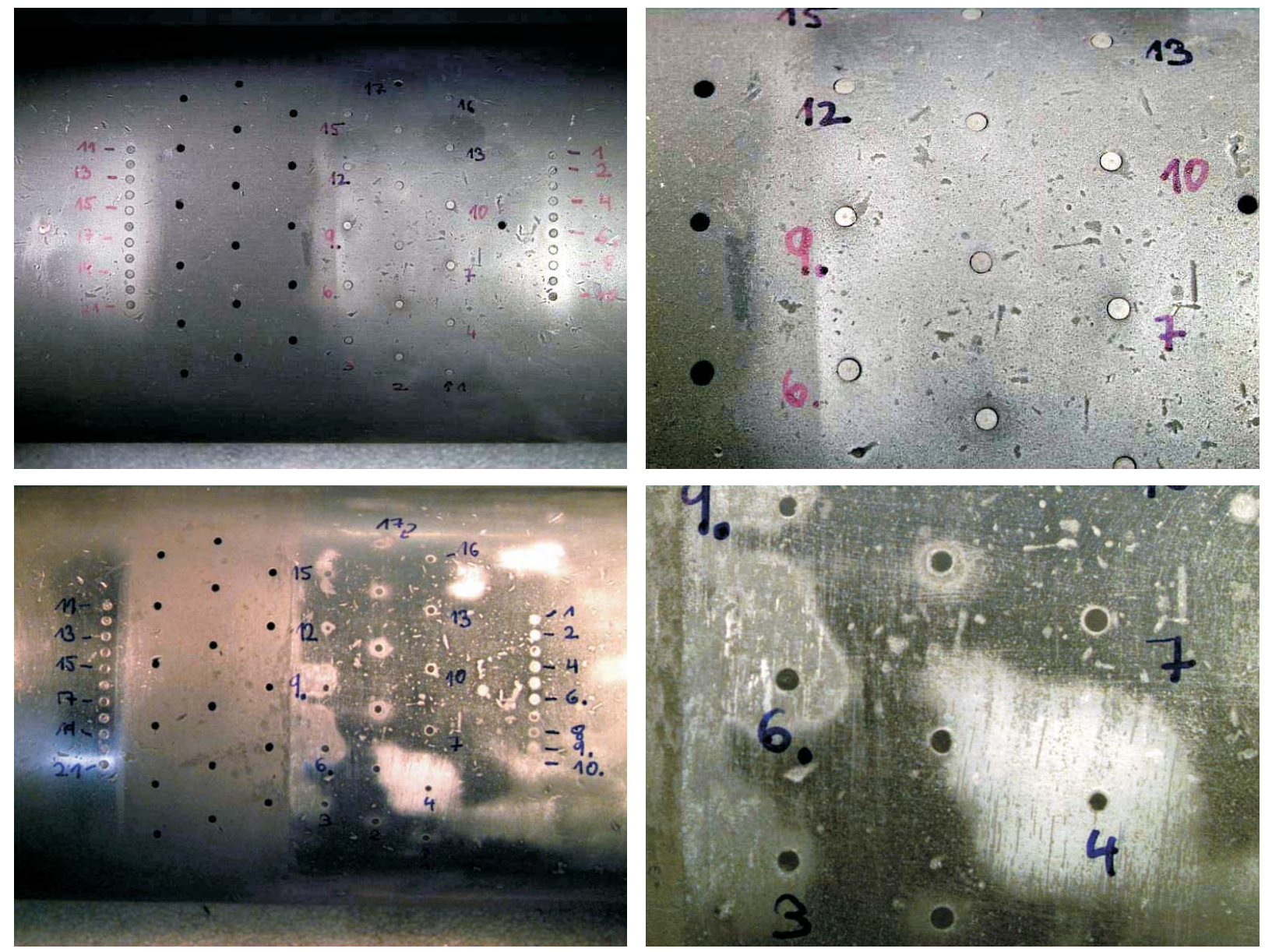

Abbildung 4.16: Photographien der Oberfläche vor und nach der Bearbeitung zur Verbesserung der Wärmestrommessungen. Die obere Reihe zeigt links die Gesamtansicht und rechts eine Detailansicht der Sensoren M4, M5 und M6 vor der Oberflächenbehandlung, die untere Reihe die gleichen Ausschnitte nach der Behandlung.

In Abb. 4.16 sind Photographien der Modelloberfläche vor und nach der Schleifbehandlung gegeben. Man erkennt deutlich, wie der Sprühspachtel die vorhandenen Unebenheiten der Modelloberfläche ausgleicht und dazu führt, dass die Zylinderoberfläche wieder eben wird. Es wurde nicht die gesamte Oberfläche des Zylinders bearbeitet. Die Drucksensoren wurden überklebt, um eine Verunreinigung der Druckanbohrungen mit dem Sprühspachtel zu vermeiden. Nur die nähere Umgebung der Oberfläche um die Thermoelementinstrumentierung wurde überarbeitet. 


\subsubsection{Doppelrampenmodell}

Das verwendete Rampenmodell ist aus Edelstahl (1.2842, 90MmCrV8, DIN 17350) gefertigt. Abb. 4.17 zeigt auf der linken Seite das Modell in der Seitenansicht. Die beiden Rampen bestehen aus Platten, an denen seitlich Halterungen befestigt sind. Diese Halterungen werden mit Durchgangsschrauben an die Seitenplatten montiert. Die Durchgangsschrauben können im nicht angezogenen Zustand in den radialen Ausfräsungen, die in Abb. 4.17 zu sehen sind, verschoben werden und erlauben so eine Anstellung der ganzen Platte. Die Anschlussstelle zwischen den beiden Rampen bildet die Drehachse, um die sich beide Rampen verstellen lassen. Das Rampenmodell wurde so in der Messstrecke platziert, dass die Drehachse auf der Düsenachse lag. Die erste Rampe kann in einem Winkelbereich von $0^{\circ}$ bis $35^{\circ}$, bezogen auf die Anströmung, justiert werden. Die zweite Rampe kann mit einem maximalen Anstellwinkel von $85^{\circ}$ einjustiert werden.

Die Unterseite des Modells bildet mit der Oberseite der ersten Rampe einen Winkel von $15^{\circ}$. Diese Vorderkante ist scharf und wurde vor jedem Versuch mit einem Schleifstein geschärft. Die Dicke der Platten beträgt $20 \mathrm{~mm}$. Die Breite des Modells beträgt $B_{\text {Rampe }}=350 \mathrm{~mm}$, die Länge der ersten Rampe beträgt $L_{1}=170 \mathrm{~mm}$ und die Länge der zweiten Rampe beträgt $L_{2}=155 \mathrm{~mm}$. Die optische Ausrichtung des Modells erfolgt mit normal zur Oberfläche angebrachten Rasierklingen.

Auf zwei Schnitten, die in einem Abstand von $10 \mathrm{~mm}$ parallel zur Mittellinie liegen, sind 14 Drucksensoren und 18 Thermoelemente installiert. Der Durchmesser der Druckanbohrungen beträgt $0.8 \mathrm{~mm}$, die Drucksensoren sind hinter der Druckanbohrung in der Platte eingesetzt. Die Einbauart wird in Abschnitt 4.2.2 beschrieben. Die Thermoelemente sind bündig zur Oberfläche eingesetzt. Eine genaue Beschreibung findet sich in Abschnitt 4.2.3.
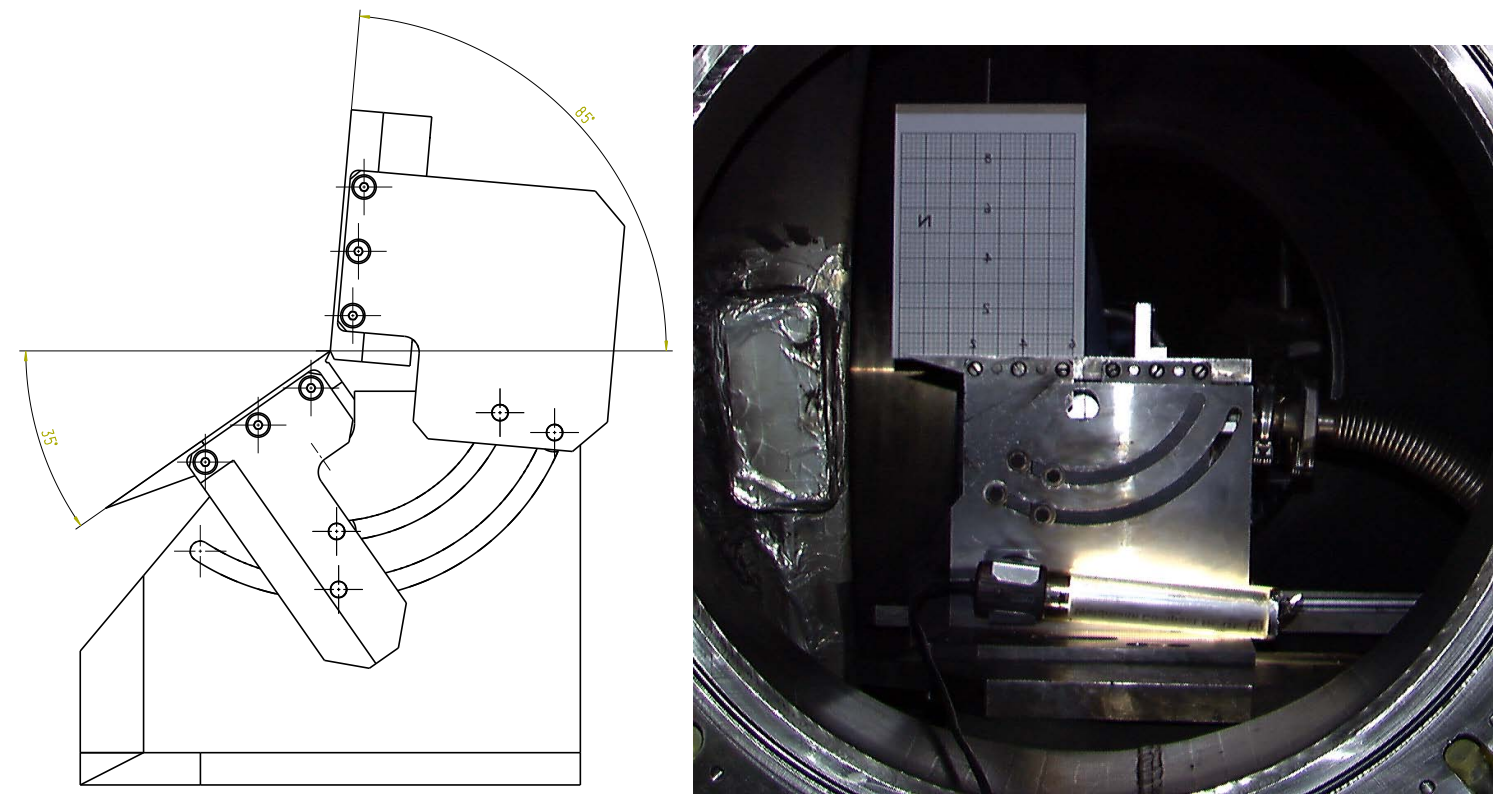

Abbildung 4.17: Doppelrampenmodell: Schematische Seitenansicht (links) und Photographie (rechts) des Modells in der HEG-Messstrecke; Auf der Photographie sind beide Rampen sind mit $0^{\circ}$ eingestellt. 


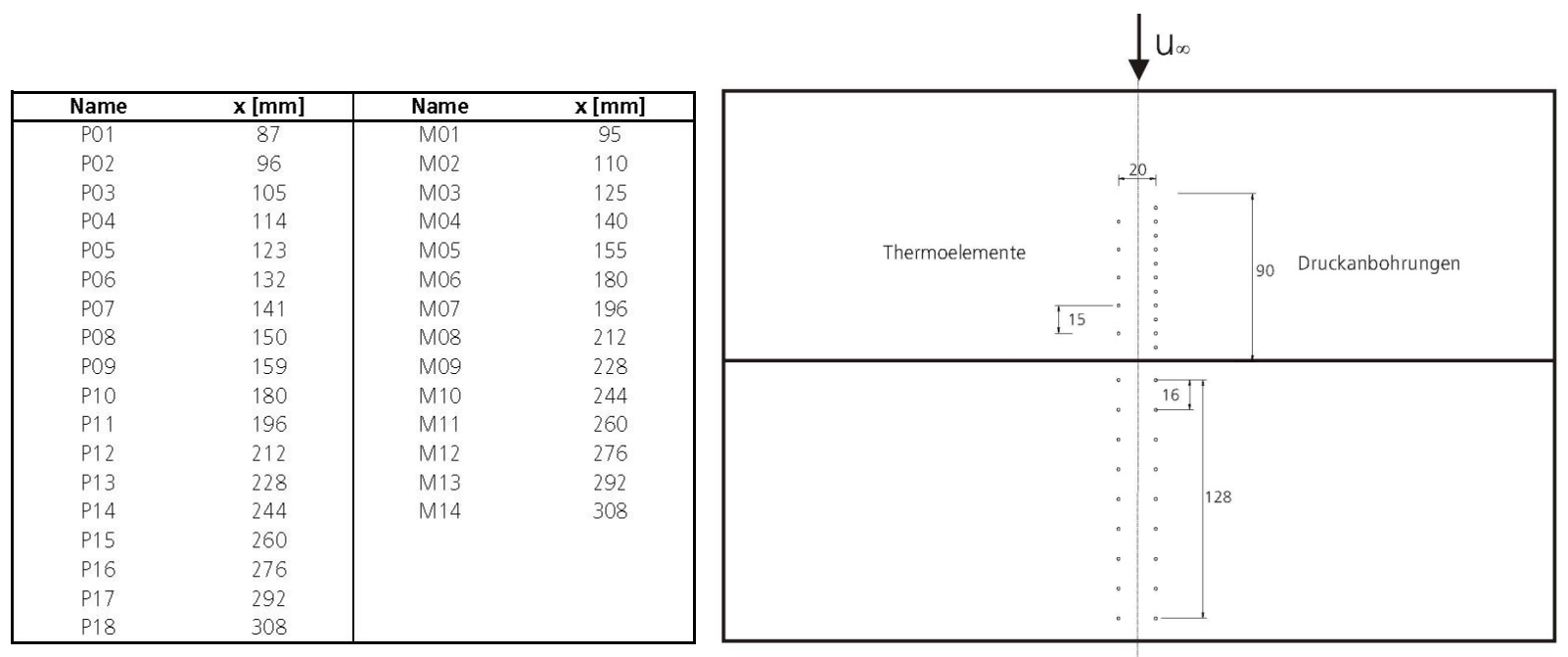

Abbildung 4.18: Die Sensorpositionen (links) bezogen auf $0^{\circ}$ Anstellung beider Rampen. Schematische Ansicht (rechts) des Doppelrampenmodells von oben. Die Anströmung erfolgt vom oberen Bildrand her. Auf der linken Seite der Mittellinie sind die Druckanbohrungen dargestellt, auf der rechte Seite die Thermoelemente.

Im Doppelrampenmodell wurden die MedTherm-Thermoelemente benutzt. Die Koordinaten, die in $\mathbf{A b b . ~} \mathbf{4 . 1 8}$ angegeben sind, beziehen sich auf eine Einstellung beider Platten mit $0^{\circ}$ Anstellwinkel.

\subsubsection{Windkanalmodell des X-38}

Das Windkanalmodell des X-38 ist aus Edelstahl (1.4021, X20Cr13, DIN17440) im Maßstab 1:24 gefertigt und wurde mit Hilfe einer gekröpften Halterung im Schwert der Messstrecke eingesetzt. Das Modell wurde von Dassault Aviation im Rahmen des NASA/ESA X-38Programmes entworfen und von AerMacci in Italien gefertigt (Labbe u. a. (1999), Tribot u. a. (1999)). Das Modell wurde bei der ONERA instrumentiert. In Abb. 4.19 ist das Modell im eingebauten Zustand in der Messstrecke dargestellt. Das Modell hat eine Länge von $300 \mathrm{~mm}$ und ist im Bereich der Flügel $220 \mathrm{~mm}$ breit.

Das Modell ist mit 46 Druckmessstellen und 141 Thermoelementen ausgerüstet. Die Druckmessstellen sind mit Abnehmerschläuchen für externe Druckaufnehmer versehen. Diese Messtechnik, ausgelegt für kontinuierlich betriebene Anlagen, konnte am HEG keine Verwendung finden. Deshalb wurde das Modell zum Teil mit neuen Drucksensoren (Kulite XCS-093 und Kulite XCE-062) ausgerüstet. Genauere Angaben über diese Sensoren und den Einbau finden sich in Abschnitt 4.2.2. Die grosse Anzahl der Sensoren, die im Modell $E$ des $X-38$ verbaut waren, konnte nicht gleichzeitig mit dem Datenaufnahmesystem des HEG vermessen werden, da die verfügbare Anzahl der Kanäle begrenzt ist. Ein Teilmenge der Sensoren wurde bestimmt. Die benutzten Sensoren sind, der besseren Übersicht halber, in Gruppen zusammengefasst. In den Versuchen wurden 2 Klappenstellungen benutzt: $20^{\circ}$ und $30^{\circ}$ Anstellung zum Vorkörper. Alle Sensorgruppen sind in Abb. 4.21 schematisch auf 

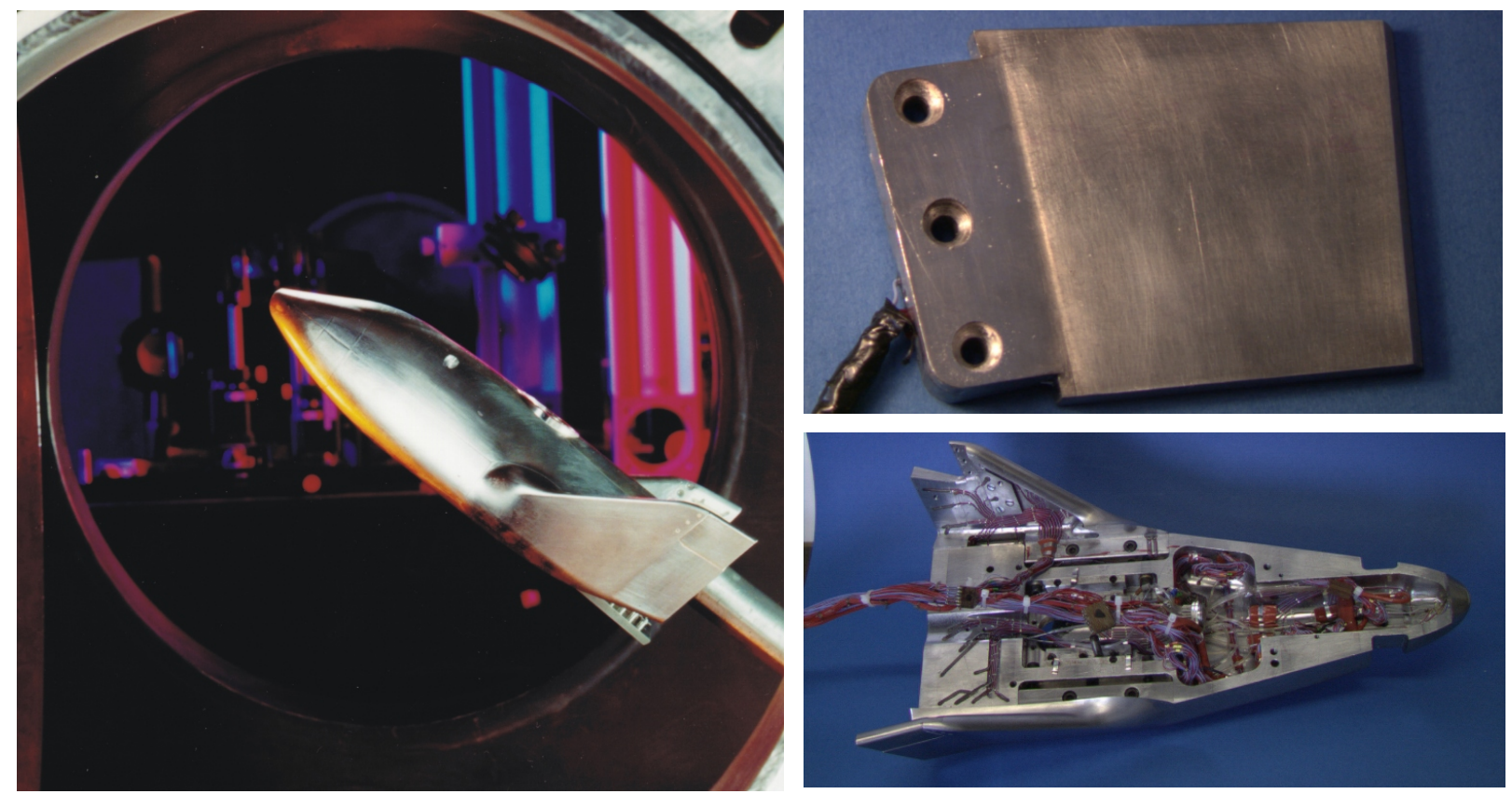

Abbildung 4.19: Photographie des X-38-Modells in der HEG-Messstrecke (links) und im geöffneten Zustand (rechts unten). Rechts oben ist eine mit Thermoelementen instrumentierte Klappe abgebildet.

der Oberfläche des Körpers dargestellt. Die exakten Koordinaten der Sensoren sind in Tab. 4.6 aufgelistet.

Die Sensorgruppe PRESS besteht aus den Untergruppen PRESSBO, PRESS20 und PRESS30. Sie vereint die verwendeten Drucksensoren entlang Schnitten, die fast parallel zur Symmetrielinie auf der Unterseite des Modells liegen. PRESSBO vereint vier Drucksensoren, die in der Symmetrielinie auf dem Vorkörper liegen. Jede der Untergruppen PRESS20 und PRESS30 enthält jeweils 11 Drucksensoren, die auf einem Schnitt parallel zur Symmetrielinie bei $y=34 \mathrm{~mm}$ liegen. Vier dieser Sensoren liegen wiederum auf dem Vorkörper, die restlichen sieben auf den jeweiligen Klappen mit $20^{\circ}$ und $30^{\circ}$ Anstellwinkel.

Die Sensorgruppe BOSY fasst insgesamt 13 Thermoelemente zusammen. Drei Thermoelemente sind in der Nase des Modelles eingesetzt, sechs Sensoren auf dem Vorkörper und vier Thermoelemente im Klappenschacht. Da die Krümmung der Oberfläche im Nasenbereich des Modelles extrem stark ist, sind hier ONERA-Thermoelemente mit einem Durchmesser von $1.7 \mathrm{~mm}$ eingesetzt. Die Sensoren auf dem Vorkörper und dem Klappenschacht haben einen Durchmesser von $2.7 \mathrm{~mm}$.

Die Sensorgruppe FLAP ist in drei Untergruppen unterteilt: FLAP20, FLAP30 und FL30SIDE. Die Untergruppe FLAP20 besteht aus 13 Thermoelementen, wobei vier Thermoelemente auf dem Vorkörper und neun Thermoelemente auf der $20^{\circ}$-Klappe platziert sind. Die Thermoelemente auf dem Vorkörper sind ONERA-Sensoren mit einem Durchmesser von $2.7 \mathrm{~mm}$. Auf der Klappe kamen Thermoelemente von MedTherm mit einem Durchmesser von $1.6 \mathrm{~mm}$ zu Einsatz. Die Untergruppe FLAP3O besteht aus den vier ONERA-Elementen auf dem Vorkörper und den neun MedTherm-Elementen auf der 30-Klappe. Die Unter- 
gruppe FL3OSIDE enthält vier Medtherm-Sensoren, die auf der Innenseite der $30^{\circ}$-Klappe verbaut sind. Die $20^{\circ}$-Klappe hat diese Sensoren nicht.

Die Sensorgruppen BOX und BOY bestehen aus den Untergruppen BOX194, BOY257 und BOY054. Die Untergruppen BOX194 und BOY257 fassen Sensoren zusammen, die auf Schnitten bei $x=194 \mathrm{~mm}$ und $x=257 \mathrm{~mm}$ senkrecht zur Symmetrieebene liegen. Die Untergruppe BOY04 liegt entsprechend auf einem Schnitt parallel zur Symmetrieebene bei $\mathrm{y}=54 \mathrm{~mm}$.

Die letzte Gruppe WING besteht aus vier Sensoren, die um die Vorderkante des Flügels positioniert sind. Diese Sensoren liegen nicht in einer Ebene und werden deshalb in der Diskussion der Messergebnisse auch nur mit Punktwerten aus den numerischen Ergebnissen verglichen.

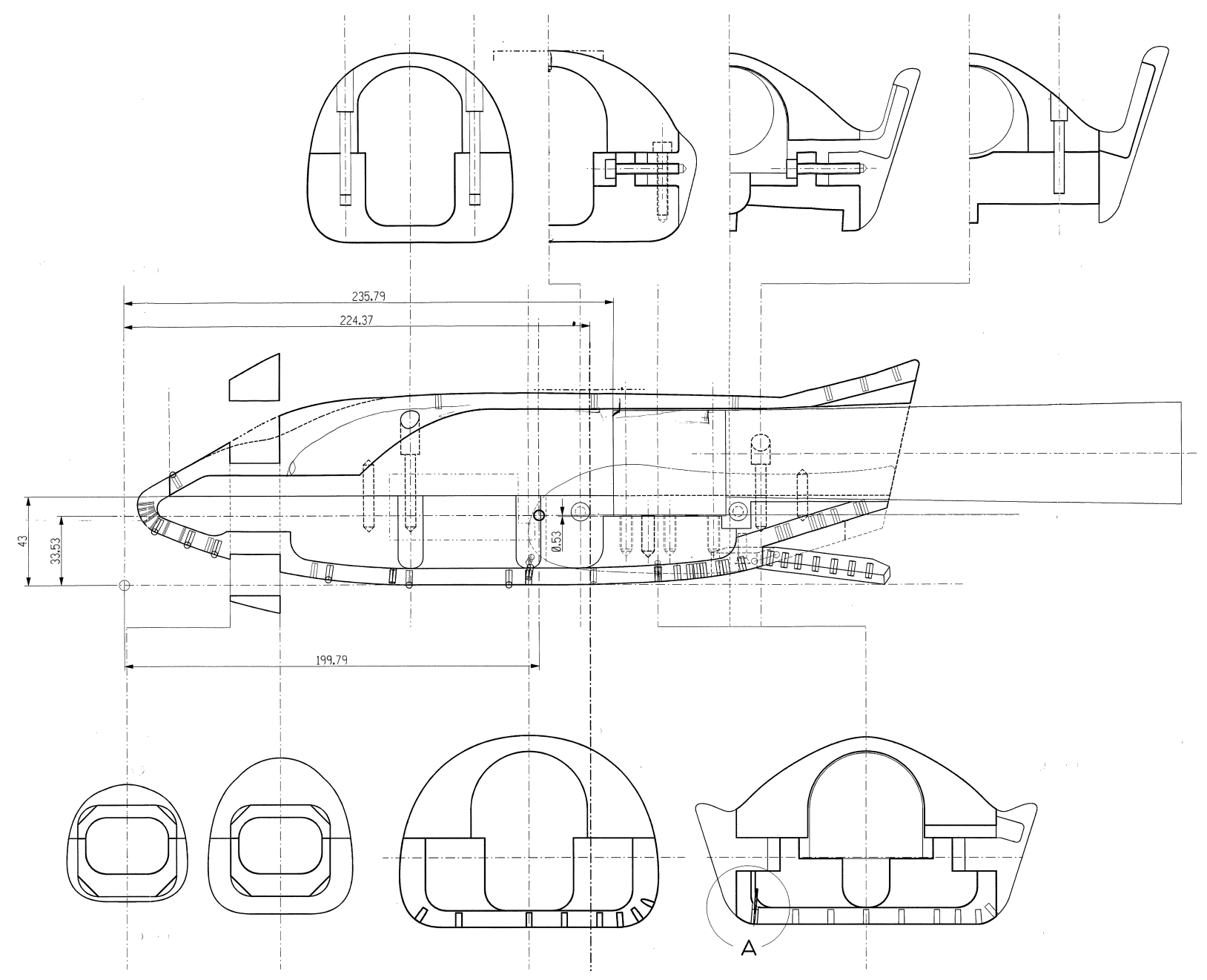

Abbildung 4.20: Schnittzeichnung des Windkanalmodells des X-38. 


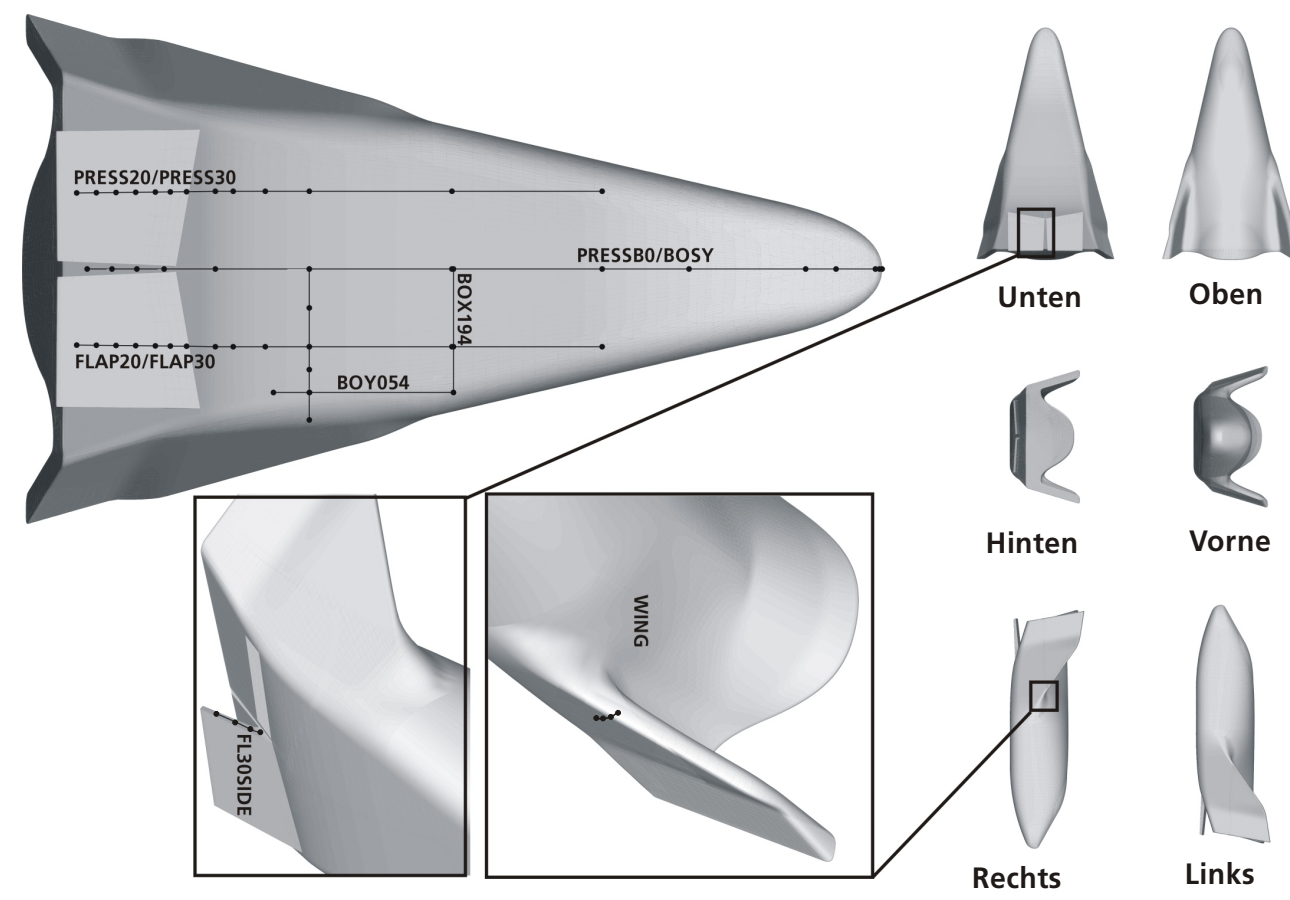

Abbildung 4.21: Schematische Darstellungen der Sensorgruppen des X-38-Modells.

\begin{tabular}{|c|c|c|c|c|c|c|c|c|c|c|c|c|c|c|}
\hline PRESSBO & 4 & Type & Kulite & & BOSY & $3+6+4$ & Typ & ONERA & & FLAP30 & $4+9$ & Type & \multicolumn{2}{|c|}{ ONERA/MedTherm } \\
\hline Name & & $x[\mathrm{~mm}]$ & $\mathrm{y}[\mathrm{mm}]$ & $\mathrm{z}[\mathrm{mm}]$ & Name & & $\mathrm{x}[\mathrm{mm}]$ & $y[\mathrm{~mm}]$ & $z[\mathrm{~mm}]$ & Name & & $\mathrm{x}[\mathrm{mm}]$ & $\mathrm{y}[\mathrm{mm}]$ & $z[\mathrm{~mm}]$ \\
\hline $\mathrm{P} 03$ & & 80.00 & 0.00 & 10.34 & G03 & & 7.53 & 0.00 & 33.49 & J01 & & 128.92 & -34.00 & 1.09 \\
\hline P06 & & 137.11 & 0.00 & 0.00 & G04 & & 9.31 & 0.00 & 30.33 & N02 & & 194.68 & -34.00 & 0.00 \\
\hline P07 & & 185.20 & 0.00 & 1.09 & G09 & & 26.53 & 0.00 & 20.85 & T02 & & 257.13 & -34.00 & 1.41 \\
\hline \multirow[t]{3}{*}{ P17 } & & 287.23 & 0.00 & 4.44 & M01 & & 39.84 & 0.00 & 16.10 & J02 & & 276.37 & -34.00 & 3.02 \\
\hline & & & & & M02 & & 90.90 & 0.00 & 3.64 & МD3T9 & & 290.43 & -34.00 & 4.90 \\
\hline & & & & & M03 & & 128.93 & 0.00 & 0.22 & МD3T8 & & 298.24 & -34.00 & 6.50 \\
\hline PRESS20 & \multirow[t]{2}{*}{$2+9$} & Type & \multicolumn{2}{|l|}{ Kulite } & M04 & & 194.70 & 0.00 & 0.00 & MD3T7 & & 310.27 & -33.64 & 8.99 \\
\hline Name & & $x[\mathrm{~mm}]$ & $\mathrm{y}[\mathrm{mm}]$ & $\mathrm{z}[\mathrm{mm}]$ & M05 & & 257.13 & 0.00 & 1.41 & МD3т6 & & 317.21 & -33.48 & 7.50 \\
\hline P05 & & 128.92 & 34 & 1.09 & M06 & & 298.24 & 0.00 & 6.54 & MD3T5 & & 323.61 & -33.32 & 6.12 \\
\hline P11 & & 257.13 & 34 & 1.41 & L03 & & 320.69 & 0.00 & 22.35 & MD3T4 & & 332.02 & -33.11 & 4.31 \\
\hline PF1 & & 290.43 & 34 & 4.96 & L04 & & 332.62 & 0.00 & 26.07 & МD3т3 & & 340.45 & -32.91 & 2.50 \\
\hline PF2 & & 298.24 & 34 & 6.5 & L08 & & 343.49 & 0.00 & 29.45 & MD3T2 & & 348.88 & -32.70 & 0.69 \\
\hline PF3 & & 310.86 & 33.77 & 9.38 & L09 & & 354.35 & 0.00 & 32.84 & \multicolumn{2}{|l|}{ MD3T1 } & 357.30 & -325.00 & -1.12 \\
\hline PF4 & & 317.95 & 33.7 & 9.09 & & & & & & FLAP20 & $4+9$ & Тур & \multicolumn{2}{|c|}{ ONERA/MedTherm } \\
\hline PF5 & & 324.49 & 33.63 & 8.82 & BOX194 & 3 & Тур & ONERA & & Name & & $\mathrm{x}[\mathrm{mm}]$ & $y[\mathrm{~mm}]$ & $z[\mathrm{~mm}]$ \\
\hline PF6 & & 333.1 & 33.53 & 8.47 & Name & & $\mathrm{x}[\mathrm{mm}]$ & $y[\mathrm{~mm}]$ & $z[\mathrm{~mm}]$ & J01 & & 128.92 & -34.00 & 1.09 \\
\hline PF7 & & 341.72 & 33.44 & 8.12 & M04 & & 194.70 & 0.00 & 0.00 & N02 & & 194.68 & -34.00 & 0.00 \\
\hline PF8 & & 350.32 & 33.35 & 7.77 & N02 & & 194.68 & -34.00 & 0.00 & T02 & & 257.13 & -34.00 & 1.41 \\
\hline \multirow[t]{3}{*}{ PF9 } & & 358.94 & 33.25 & 7.41 & N04 & & 194.68 & -54.00 & 3.15 & 102 & & 276.37 & -34.00 & 3.02 \\
\hline & & & & & & & & & & MDT9 & & 290.43 & -34.00 & 4.96 \\
\hline & & & & & BOX257 & 6 & Tyр & ONERA & & MDT8 & & 298.24 & -34.00 & 6.50 \\
\hline $\begin{array}{l}\text { PRESS30 } \\
\end{array}$ & \multirow[t]{2}{*}{$2+9$} & Type & \multirow{2}{*}{$\begin{array}{l}\text { Kulite } \\
y[\mathrm{~mm}]\end{array}$} & \multirow[b]{2}{*}{$\mathrm{z}[\mathrm{mm}]$} & Name & & $\mathrm{x}[\mathrm{mm}]$ & $y[\mathrm{~mm}]$ & $z[\mathrm{~mm}]$ & \multirow{2}{*}{\multicolumn{2}{|c|}{$\begin{array}{l}\text { MDT7 } \\
\text { MDT6 }\end{array}$}} & 310.86 & -33.77 & 9.38 \\
\hline Name & & $x[\mathrm{~mm}]$ & & & M05 & & 257.13 & 0.00 & 1.41 & & & 317.95 & -33.70 & 9.09 \\
\hline P05 & & 128.92 & 34.00 & 1.09 & T01 & & 257.13 & -17.00 & 1.41 & \multicolumn{2}{|l|}{ MDT5 } & 324.49 & -33.63 & 8.82 \\
\hline P11 & & 257.13 & 34.00 & 1.41 & T02 & & 257.13 & -34.00 & 1.41 & \multicolumn{2}{|l|}{ MDT4 } & 333.11 & -33.53 & 8.47 \\
\hline P3F1 & & 290.43 & 34.00 & 4.96 & Т03 & & 257.13 & -44.00 & 1.41 & \multicolumn{2}{|l|}{ MDT3 } & 341.72 & -33.44 & 8.12 \\
\hline P3F2 & & 298.24 & 34.00 & 6.50 & T04 & & 257.13 & -54.00 & 1.41 & \multicolumn{2}{|l|}{ MDT2 } & 350.32 & -33.35 & 7.77 \\
\hline Р3F3 & & 310.27 & 33.64 & 8.99 & T06 & & 257.13 & -66.99 & 10.83 & \multicolumn{2}{|l|}{ MDT1 } & 358.94 & -33.26 & 7.41 \\
\hline P3F4 & & 317.21 & 33.48 & 7.50 & & & & & & \multirow{2}{*}{\multicolumn{2}{|c|}{$\begin{array}{c}\text { FL30SIDE } \\
\text { Name }\end{array}$}} & Tyр & \multicolumn{2}{|l|}{ MedTherm } \\
\hline P3F5 & & 323.61 & 33.32 & 6.12 & BOY054 & 3 & Typ & ONERA & & & & $\mathrm{x}[\mathrm{mm}]$ & $\mathrm{y}[\mathrm{mm}]$ & $z[\mathrm{~mm}]$ \\
\hline P3F6 & & 332.02 & 33.11 & 4.31 & Name & & $\mathrm{x}[\mathrm{mm}]$ & $y[\mathrm{~mm}]$ & $z[\mathrm{~mm}]$ & \multirow{2}{*}{\multicolumn{2}{|c|}{$\begin{array}{l}\text { FS1 } \\
\text { FS2 }\end{array}$}} & 354.77 & -2.38 & 4.82 \\
\hline P3F7 & & 340.45 & 32.91 & 2.50 & N04 & & 194.68 & -54.00 & 3.15 & & & 343.12 & -2.06 & 7.68 \\
\hline P3F8 & & 348.88 & 32.70 & 0.69 & T04 & & 257.13 & -54.00 & 1.41 & \multicolumn{2}{|l|}{ FS3 } & 331.47 & -1.74 & 10.54 \\
\hline P3F9 & & 357.30 & 32.50 & -1.12 & $\mathrm{~K} 02$ & & 272.81 & -54.00 & 2.65 & \multicolumn{2}{|l|}{ FS4 } & 319.82 & -1.42 & 13.41 \\
\hline
\end{tabular}

Tabelle 4.6: Koordinaten der Sensoren des X-38-Modells: PRESS: Drucksensoren auf Vorkörper/Klappen; BOSY: Thermoelemente in Symmetrielinie/Klappenschacht; FLAP: Thermoelemente parallel zur Symmetrielinie auf Vorkörper/Klappe; BOXIBOY Thermoelemente auf Vorkörper; WING: Thermoelemente auf Flügelnase; FL30SIDE: Thermoelemente auf der Klappeninnenseite der $30^{\circ}$ Klappe. 


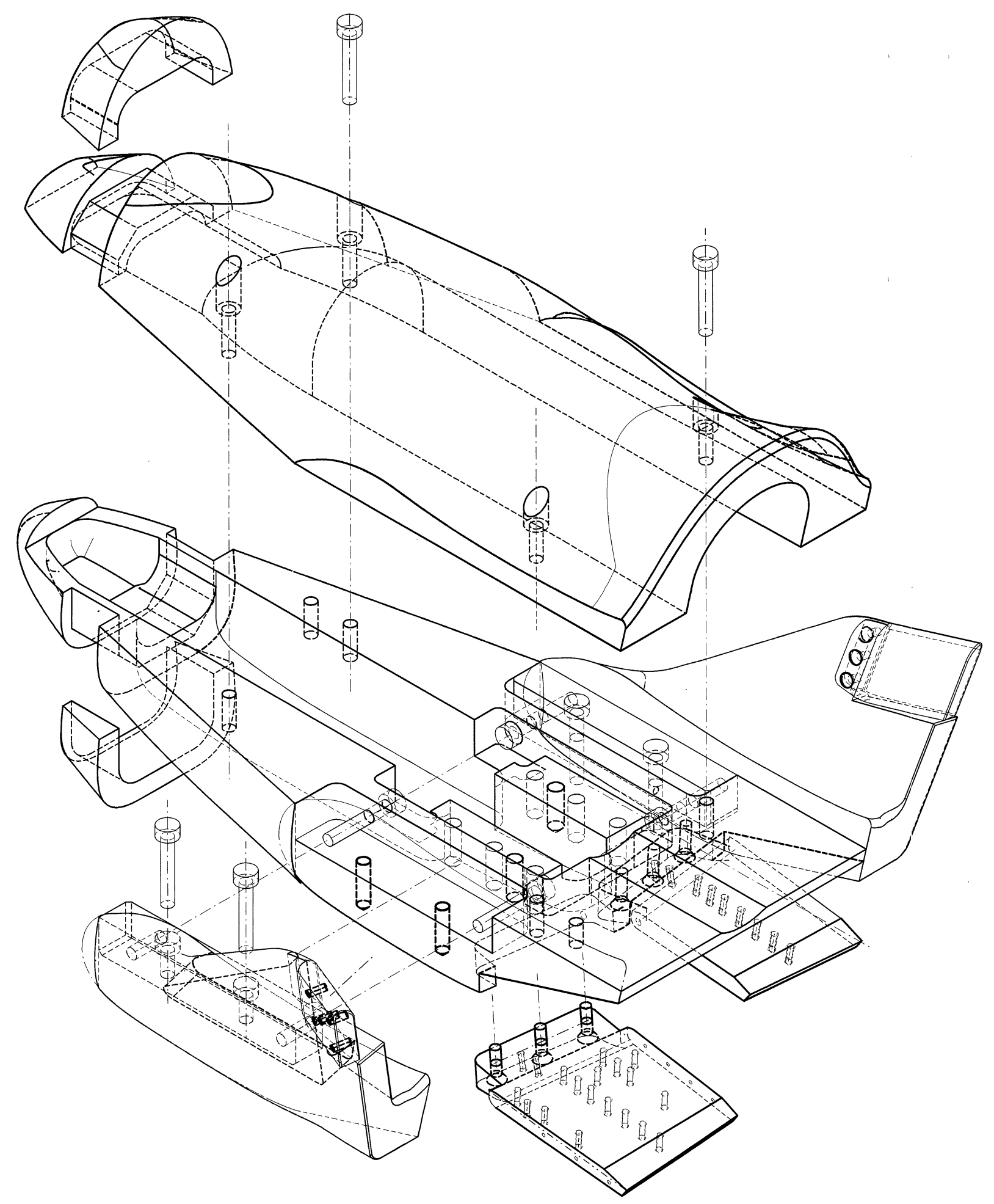

Abbildung 4.22: Explosionszeichnung des Windkanalmodells des $X$-38 


\section{Diskussion der Messergebnisse}

\subsection{Zylindermodell}

Die Versuche mit dem Zylindermodell sind in Tab. 5.1 aufgelistet. Die Zylinderumströmung wurde für die HEG-Versuchsbedingungen I und III mit Luft und Stickstoff als Testgas vermessen. Begleitend zu den Versuchen wurden numerische Simulationen der Düsenströmung von Karl u. a. (2003) durchgeführt. Für die HEG-Bedingungen I und III mit Luft und die HEG-Bedingung I mit Stickstoff als Versuchsgas zeigte die Reaktionsratenmodellierung nach Gupta u.a. (1990) die beste Übereinstimmung. Bei der HEG-Bedingung III mit Stickstoff als Versuchsgas ergab die Modellierung nach Park (1985) die beste Übereinstimmung. Da die numerischen Ergebnisse der freien Anströmung in die Zylinderrechnungen eingehen, wurde das Reaktionsratenmodell konsequenterweise auch für die Zylinderrechnungen beibehalten. Es wurden Rechnungen der Zylinderumströmung für die Versuche 615, 619, 625 und 627 durchgeführt.

\begin{tabular}{|c|c|c|c|c|c|c|}
\hline Versuch & Bedingung & Testgas & Visualisierung & Aufnahmezeit [ms] (SR) & CFD & Reaktionratenmodell \\
\hline 615 & III & $\mathrm{N}_{2}$ & $\mathrm{HI}$ & 2.731 & $\mathrm{O}$ & Park \\
\hline 617 & III & $\mathrm{N}_{2}$ & $\mathrm{HI}$ & 2.801 & & \\
\hline 618 & 1 & Luft & $\mathrm{HI}$ & 2.314 & & \\
\hline 619 & I & Luft & $\mathrm{HI}$ & 1.992 & O & Gupta \\
\hline 620 & I & Luft & $\mathrm{HI}$ & 2.474 & & \\
\hline 621 & I & Luft & $\mathrm{HI}$ & 2.477 & & \\
\hline 625 & I & $\mathrm{N}_{2}$ & $\mathrm{HI}$ & 1.444 & O & Gupta \\
\hline 626 & I & $\mathrm{N}_{2}$ & $\mathrm{HI}$ & 2.402 & & \\
\hline 627 & III & Luft & $\mathrm{HI}$ & 2.523 & O & Gupta \\
\hline 628 & III & Luft & $\mathrm{HI}$ & 2.501 & & \\
\hline 611 & III & Luft & HSFV & $0.993-4.629[15 \mathrm{kHz}]$ & & \\
\hline 632 & I & Luft & HSFV & $1.108-4.180[15 \mathrm{kHz}]$ & & \\
\hline
\end{tabular}

Tabelle 5.1: Zylinderversuche. HI=Holographische Interferometrie; HSFV=HochgeschwindigkeitsSichtbarmachung.

Um die mit Hilfe der holographischen Interferometrie gemessenenen Phasenverschiebungen mit den numerischen Ergebnissen zu vergleichen, war es erforderlich, wie in Abschnitt 5.1.3 diskutiert wird, die Rechnungen dreidimensional durchzuführen. Die Ortsauflösung der strukturierten Netze im Bereich des Verdichtungsstoßes musste aufwendig erhöht (adaptiert) werden, um mittels eines Strahlverfolgungsprogrammes numerische Interferenzbilder zu erzeugen, die für Vergleiche mit den experimentellen Ergebnissen geeignet sind. Diese dreidimensionalen Rechnungen sind zeitlich so aufwendig (2 Wochen reine CPU-Zeit auf einer modernen Workstation), dass es nicht möglich war, für alle Versuche mehr numerische Vergleichsdaten zu erstellen.

\subsubsection{Oberflächenmessungen}

Die Messungen auf der Oberfläche des Zylinders liegen in Umfangsrichtung nicht auf einem Schnitt. In Abb. 4.15 in Abschnitt 4.3.1 sind die Positionen der Druckanbohrungen 


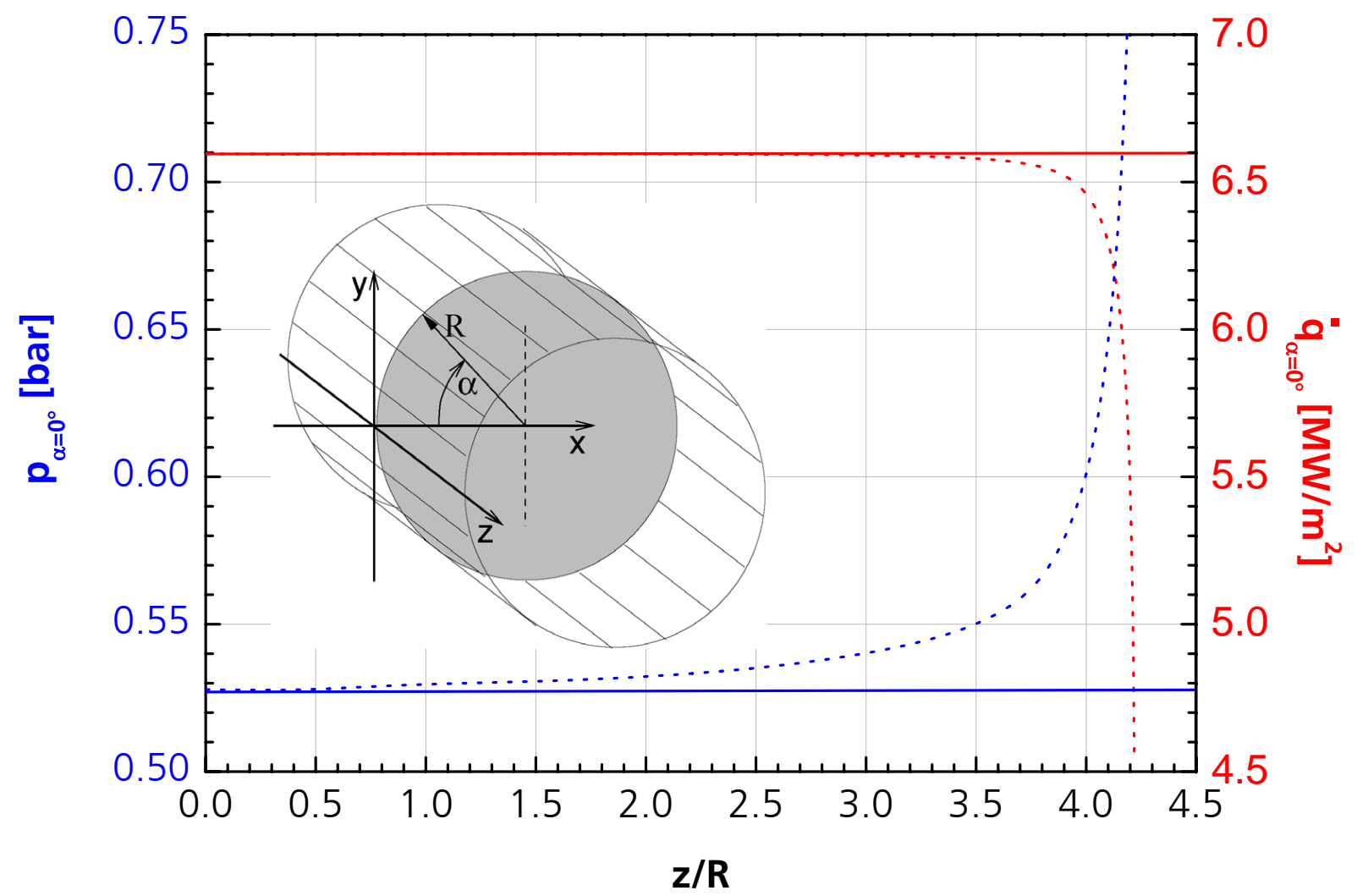

Abbildung 5.1: Einfluss der Dreidimensionalität der Strömung auf die Oberflächenmessungen.

und der Thermoelemente schematisch in einer Ansicht von vorn auf den Zylinder dargestellt. Die nachfolgenden Messungen werden trotzdem nur über den Umlaufwinkel $\alpha$ aufgetragen, da sich die Oberflächenwerte innerhalb von $z / R<0.6$ in Spannweitenrichtung nicht unterscheiden. Diese Aussage resultiert aus einem Vergleich der zweidimensionalen und dreidimensionalen numerischen Rechnungen der Zylinderumströmung. In Abb. $\mathbf{5 . 1}$ sind diese Unterschiede für den Oberflächendruck und den Wandwärmeübergang entlang der z-Achse aufgetragen. Für die zweidimensionalen Rechnungen wurde nur eine Linie gezeichnet. Ein deutlicher Unterschied ab einem z/R von 0.8 für den Druck und 3.5 für den Wärmeübergang ist erkennbar. Da sich die Instrumentierung in dem Bereich bis $z / R<0.6$ befindet, ist für die vorgestellten Ergebnisse der Oberflächenmessungen kein Einfluss zu erwarten.

In der Abb. $\mathbf{5 . 2}$ sind die gemessenen Druckverläufe auf der Zylinderoberfläche aufgetragen. Die Messpunkte stellen die Mittelwerte der Zeitsignale innerhalb der in Tab. 3.3 angegebe-

\begin{tabular}{|ccc|cccccc|}
\hline Versuch & Bedingung & $\begin{array}{c}\text { Experiment } \\
\mathbf{p}_{\alpha=0^{\circ}}[\text { Gar] }\end{array}$ & $\begin{array}{c}\text { Gupta } \\
\mathbf{p}_{\alpha=0^{\circ}}[\text { bar] }\end{array}$ & Abw. & $\begin{array}{c}\text { Dunn und Kang } \\
\mathbf{p}_{\alpha=0^{\circ}}[\text { bar] }\end{array}$ & Abw. & $\begin{array}{c}\text { Park } \\
\mathbf{p}_{\alpha=0^{\circ}}[\text { bar] }\end{array}$ & Abw. \\
\hline 627 & III, Luft & 0.666 & 0.710 & $6.2 \%$ & 0.706 & $5.7 \%$ & 0.707 & $5.8 \%$ \\
615 & III, N2 & 0.634 & 0.679 & $6.6 \%$ & 0.671 & $5.5 \%$ & 0.667 & $4.9 \%$ \\
619 & I, Luft & 0.507 & 0.529 & $4.2 \%$ & 0.523 & $3.1 \%$ & 0.519 & $2.3 \%$ \\
625 & $\mathrm{I}, \mathrm{N}_{2}$ & 0.525 & 0.485 & $-8.2 \%$ & 0.490 & $-7.1 \%$ & 0.486 & $-8.0 \%$ \\
\hline
\end{tabular}

Tabelle 5.2: Vergleich der Zylinder-Staupunktdrücke zwischen Experiment und Numerik. 


\begin{tabular}{|c|c|c|c|c|c|c|c|c|}
\hline \multirow[t]{2}{*}{ Versuch } & \multirow[t]{2}{*}{ Bedingung } & \multirow{2}{*}{$\begin{array}{c}\text { Experiment } \\
q_{\alpha=0^{\circ}}\left[\mathrm{MW} / \mathrm{m}^{2}\right]\end{array}$} & \multirow{2}{*}{$\begin{array}{l}\text { Gupta } \\
\mathrm{q}_{\alpha=0^{\circ}}\left[\mathrm{MW} / \mathrm{m}^{2}\right]\end{array}$} & \multirow[b]{2}{*}{ Abw. } & \multicolumn{2}{|l|}{ Dunn und Kang } & \multicolumn{2}{|l|}{ Park } \\
\hline & & & & & $\mathbf{q}_{\alpha=0^{\circ}}\left[\mathrm{MW} / \mathrm{m}^{2}\right]$ & Abw. & $q_{\alpha=0^{\circ}}\left[\mathrm{MW} / \mathrm{m}^{2}\right]$ & Abw. \\
\hline 627 & III, Luft & 4.566 & 4.790 & $4.7 \%$ & 4.780 & $4.5 \%$ & 4.770 & $4.3 \%$ \\
\hline 615 & III, N & 4.481 & 4.660 & $3.8 \%$ & 4.670 & $4.0 \%$ & 4.580 & $2.2 \%$ \\
\hline 619 & I, Luft & 7.342 & 7.210 & $-1.8 \%$ & 7.180 & $-2.3 \%$ & 7.060 & $-4.0 \%$ \\
\hline 625 & I, $\mathrm{N}_{2}$ & 5.985 & 6.800 & $12.0 \%$ & 6.790 & $11.9 \%$ & 6.670 & $10.3 \%$ \\
\hline
\end{tabular}

Tabelle 5.3: Vergleich der Staupunktswärmeströme zwischen Experiment und Numerik.

nen Messzeitfenster dar. Für jeden Sensor ist die Standardabweichung für diesen Mittelwert aufgetragen. Diese Standardabweichung ist als Größe für die zeitliche Schwankung des Drucksignals innerhalb des Messzeitfensters zu interpretieren. Für die Versuche mit Luft wurde der Zylinder in seiner Halterung um $20^{\circ}$ in positive sowie in negative Richtung rotiert, um einen größeren Winkelbereich abzudecken. Bei den Versuchen mit Stickstoff wurde nur jeweils eine Winkeleinstellung benutzt. Die zeitliche Schwankung der Signale liegt im Bereich von $0^{\circ}$ bei $7 \%$ und ab einem Winkel von $40^{\circ}$ bei ca. $4 \%$. Der Vergleich mit den numerischen Ergebnissen für die vier Einzelversuche ist in der Abb. 5.2 angegeben. Der Einfluss des verwendeten Reaktionsratenmodells auf die numerischen Staupunktswerte ist aus der Tab. 5.2 ersichtlich. Die numerischen Werte, die grau hinterlegt sind, entsprechen den in Abb. 5.2 aufgetragenen Kurven. Die experimentellen Staupunktswerte in Tab. 5.2 sind die Staupunktswerte, die sich aus den in Abb. 5.2 gegebenen Interpolationen der Messpunkte ergeben. Die numerischen Ergebnisse liegen bis auf Versuch 625 (Bed. I, $\mathrm{N}_{2}$ ) höher als die experimentellen Ergebnisse, die größte Abweichung beträgt 6.2\%. Die Änderung des Drucks im Staupunkt des Zylinders bei verschiedenen Reaktionsratenmodellen ist kleiner als die Abweichung vom Experiment. Es kann also festgestellt werden, dass eine Messung des Oberflächendruckes nicht geeignet ist, eine Validierung des numerischen Verfahrens bezüglich der chemischen und thermischen Modellierung zu ermöglichen.

Die Ergebnisse für den Wärmestrom in die Oberfläche des Zylinders sind in der Abb. $\mathbf{5 . 3}$ dargestellt. Die Qualität der Ergebnisse erreicht nicht die der Druckmessungen: nicht reproduzierbare lokale Maxima und Minima treten auf. Aufgrund der sehr dünnen Grenzschicht (ca. $0.1 \mathrm{~mm}$ im Staupunkt) können kleinste Unebenheiten oder Störungen in der Oberfläche des Zylinders die Wärmestrommessungen empfindlich stören. Frühere Messungen von Reimann (2000) mit dem verwendeten Modell zeigten nicht verwertbare Ergebnisse. Um die hier diskutierten Messungen zu erhalten, war eine mechanische Überarbeitung der Oberfläche vor jedem Versuch notwendig (s. Abschnitt 4.3.1). Ein Vergleich zwischen Experiment und Rechnung der Werte für den Wärmestrom im Staupunkt des Zylinders ist in Tab. $\mathbf{5 . 3}$ gegeben. Die numerischen Vorhersagen für den Wärmestrom im Staupunkt des Zylinders sind bis auf die Rechnung für Versuch 619 (Bed. I, Luft) zu hoch. Die größte Abweichung von 12\% ergibt sich für Versuch 625 (Bed. I, $N_{2}$ ). Wie bei den Ergebnissen für den Druck im Staupunkt sind die Unterschiede, die die unterschiedlichen Modellierungen ergeben, deutlich kleiner als die Abweichung vom Experiment. 

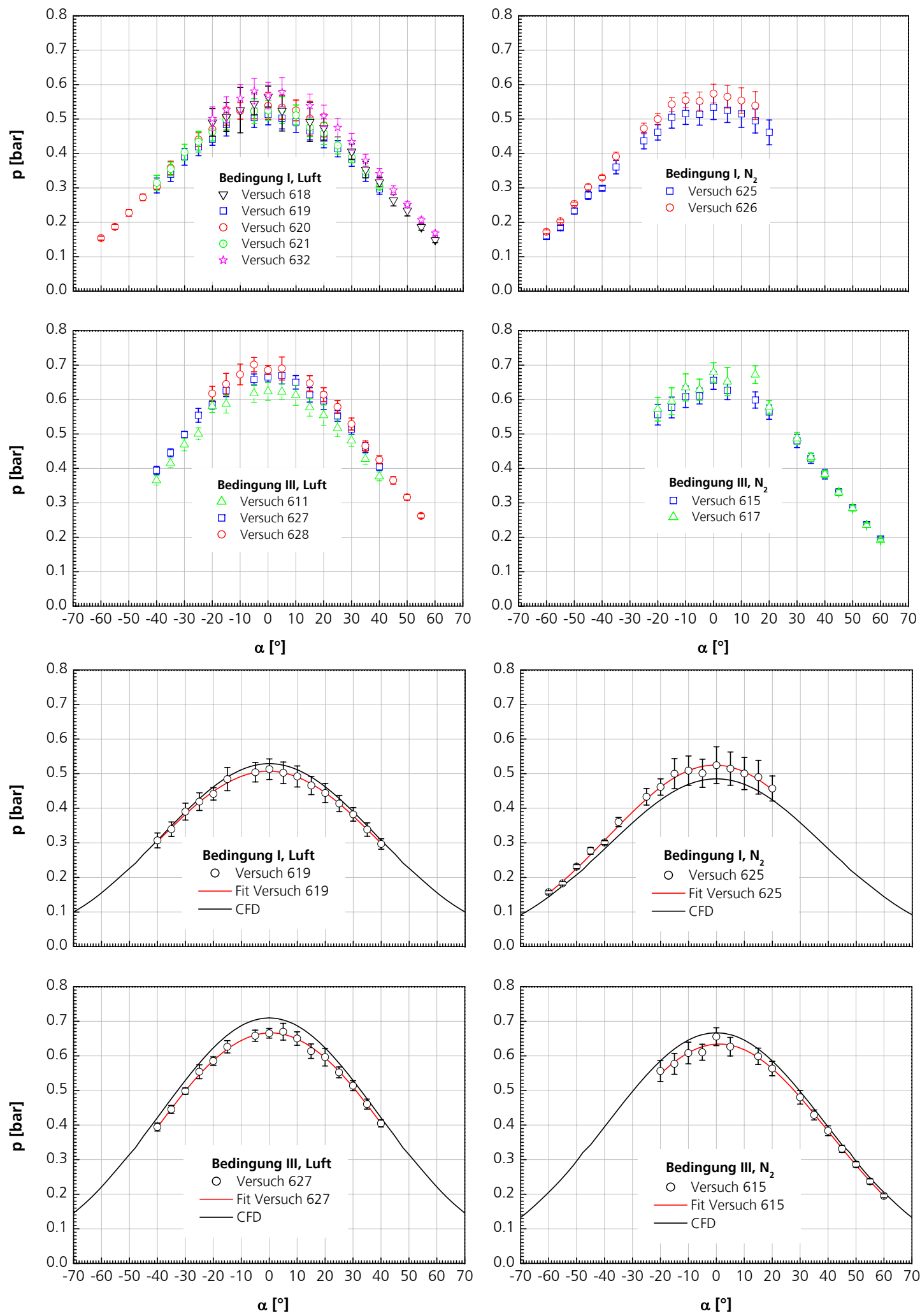

Abbildung 5.2: Ergebnisse der Druckmessung auf dem Zylinder. 

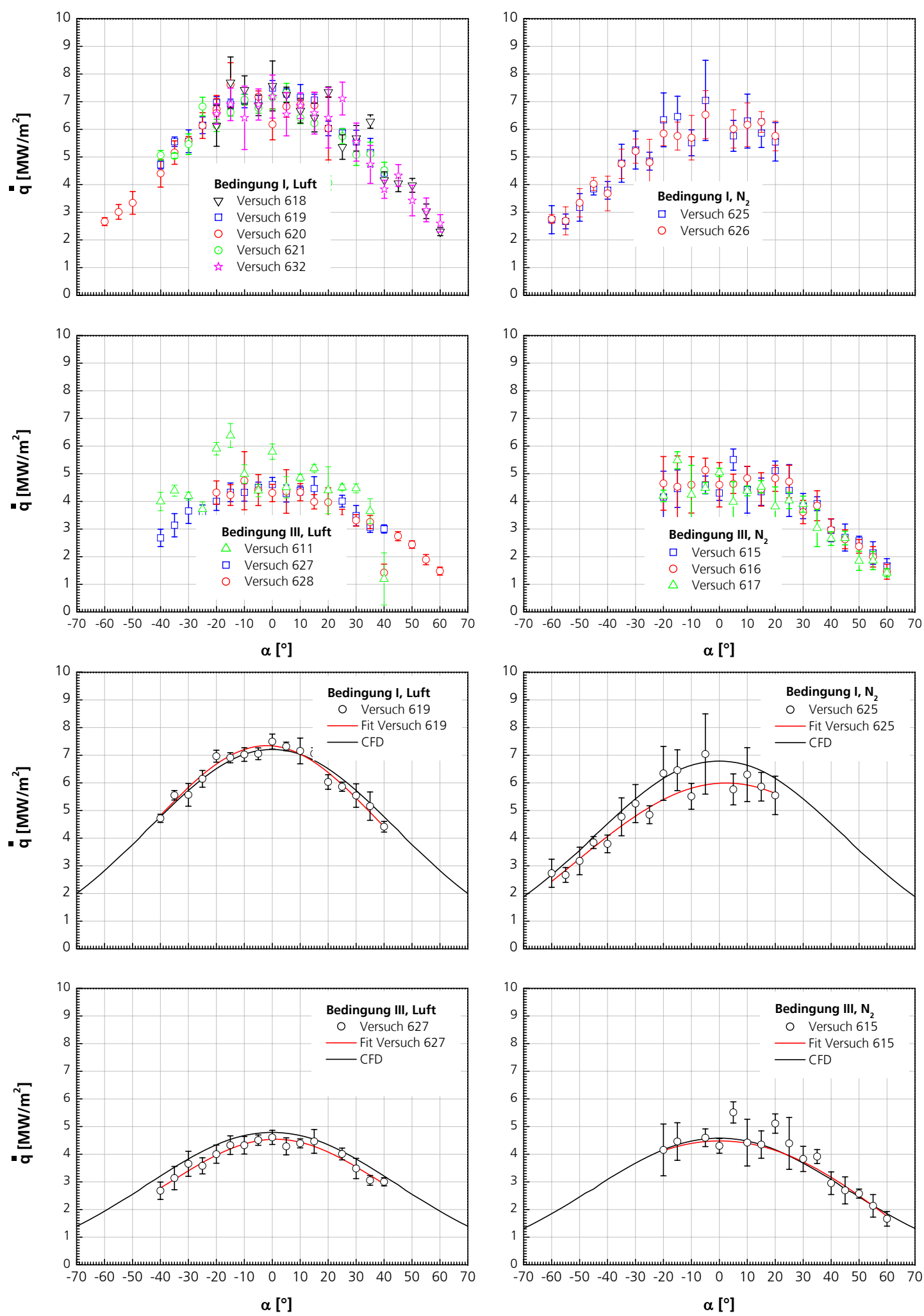

Abbildung 5.3: Wärmestrommessungen auf dem Zylinder. 


\subsubsection{Hochgeschwindigkeits-Sichtbarmachung der Zylinderumströmung}

Bei den Versuchen 611 und 632 wurde die zeitliche Entwicklung der Zylinderumströmung mit Hilfe der Hochgeschwindigkeits-Sichtbarmachung (s. Abschnitt 4.1.1) aufgezeichnet. Bei Benutzung dieses Messverfahrens kann im Vergleich zu einem Einzelbildverfahren sichergestellt werden, dass sich die untersuchte Strömung in einem stationären Zustand befindet. In der Abb. $\mathbf{5 . 4}$ ist eine Sequenz aus Schlierenbildern, die die zeitliche Entwicklung der Zylinderumströmung zeigt, gegeben. Auf der linken Seite der Abb. 5.4 ist das Strömungsbild zur Messzeit zusammen mit der Stoßlage aus der Numerik gezeigt.

Aus den Einzelbildern lässt sich der Stoßabstand auf der Staustromlinie in Abhängigkeit von der Messzeit bestimmen. Der aus den Einzelbildern ermittelte Stoßabstand ist in Abb. $\mathbf{5 . 5}$ rechts für die Bedingung III und links für die Bedingung I dargestellt.

Der Verlauf des Stoßabstandes und das dimensionslosen Verhältnis $\sqrt{p_{t 2} / p_{\infty}} \sim M$ sind dargestellt. Wie in Abschnitt 3.3.5 diskutiert, ist ein Ansteigen dieses Verhältnisses ein Hinweis auf die Ankunft von Treibgas in der Messstrecke. Für die Bedingung III zeigt dieses Verhältnis ab 2.4 ms bis 3.4 ms nach Stoßreflektionszeitpunkt einen konstanten Verlauf. Auch der zeitliche Verlauf des Stoßabstands zeigt keine Änderung. Für die Untersuchungen
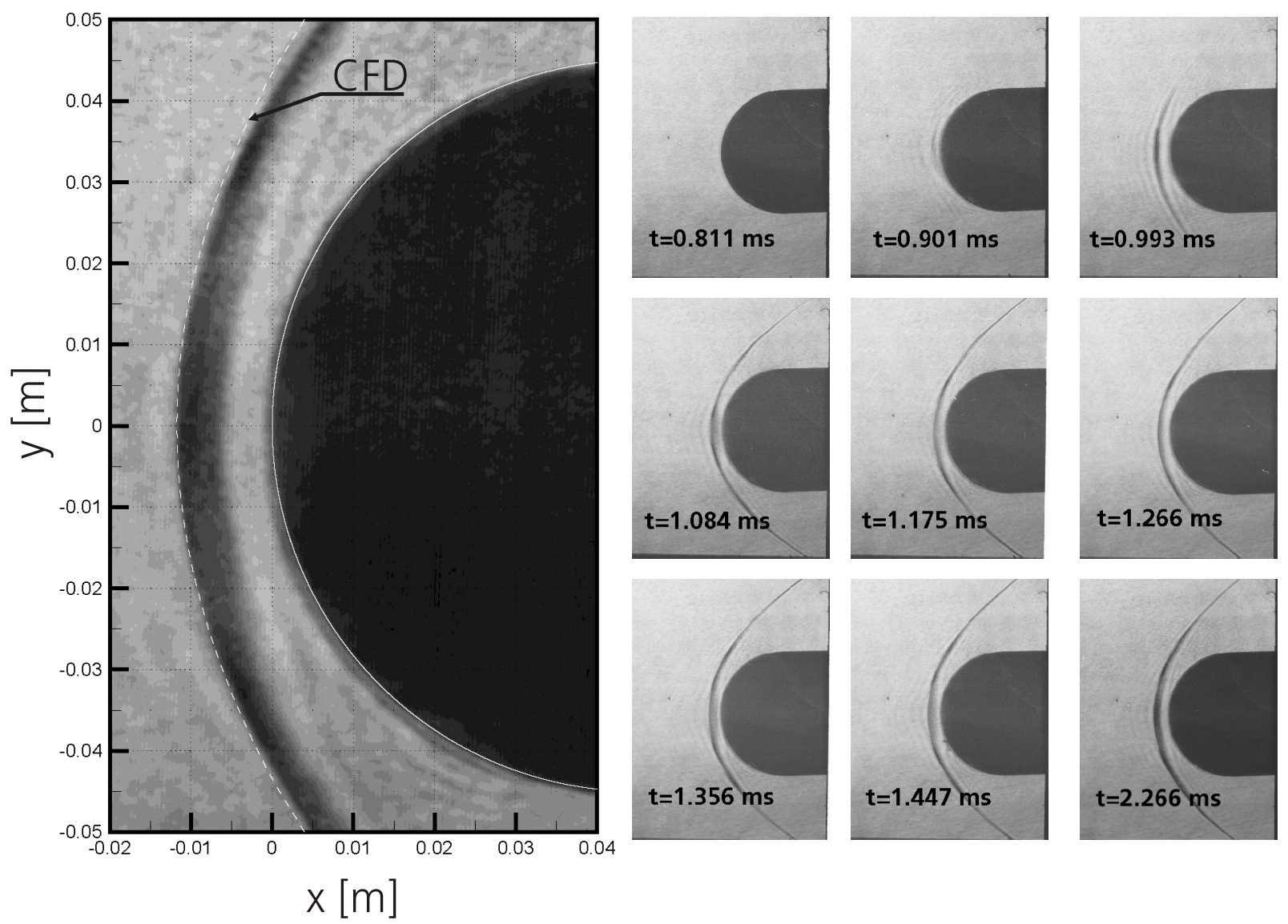

Abbildung 5.4: Zeitliche Sichtbarmachung der Zylinderumströmung bei Bedingung III, links der Vergleich zu der Stoßlage aus der numerischen Lösung mit in das Schlierenbild eingetragen. 

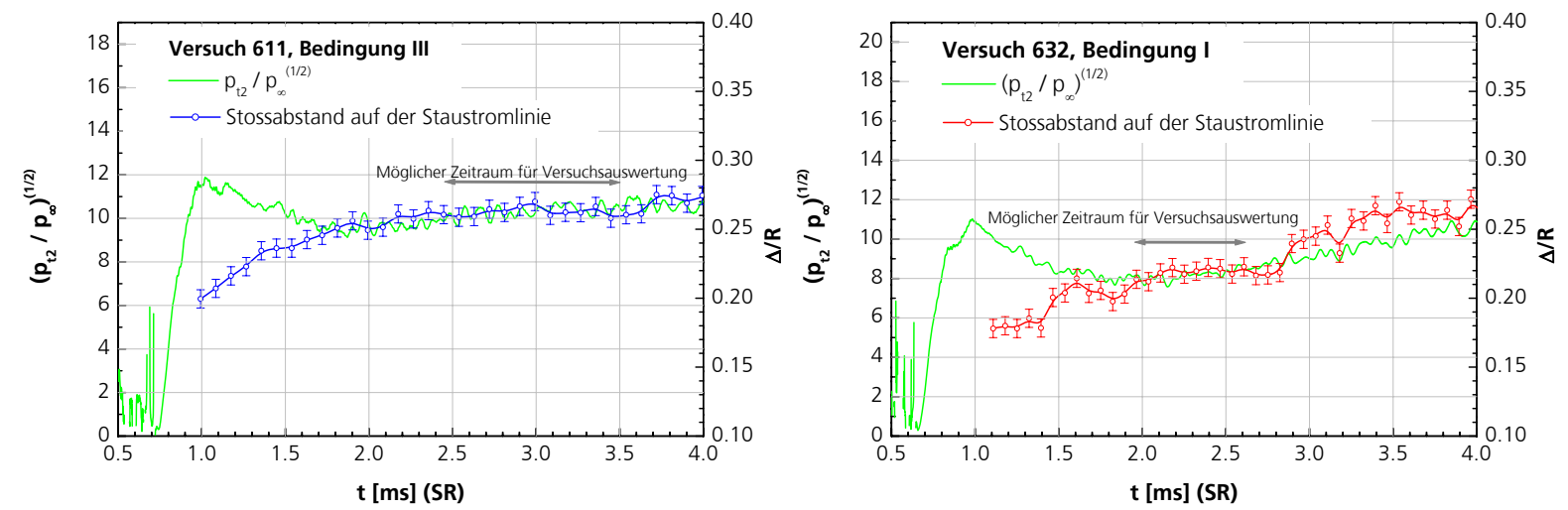

Abbildung 5.5: Zeitlicher Verlauf des Stoßabstandes auf der Staustromlinie des Zylinders bei Bedingung III (links) und Bedingung I (rechts). Zusätzlich ist der zeitliche Verlauf von $\sqrt{p_{t 2} / p_{\infty}} \sim M$ aufgetragen.

bei der Versuchsbedingung III kann also von einem konstanten Strömungszustand bei der Zylinderumströmung ausgegangen werden.

Eine anderes Verhalten tritt bei der Versuchsbedingung I auf, wie in der Abb. 5.5 auf der rechten Seite dargestellt. Hier ist zwar ein konstantes Verhältnis $p_{t 2} / p_{\infty}$ ab 1.9 ms nach Stoßreflektion zu beobachten, auch der Stoßabstand ändert sich ab diesem Zeitpunkt nicht mehr. Ab 2.6 ms nach Stoßreflektion ist allerdings ein deutliches Ansteigen sowohl des Stoßabstandes als auch des Verhältnisses $p_{t 2} / p_{\infty}$ zu beobachten. Dieses Ergebnis ist konform zu den Untersuchgen von Hannemann u. a. (2000), die die Wirkungsweise des Heliumabscheiders im HEG untersucht haben (s.a. Abschnitt 3.3.4). Ab einer Zeit 2.6 ms nach der Stoßreflektion kann keine Versuchsauswertung mehr stattfinden, da eine Heliumverunreinigung der Strömung vorliegt. Alle gewählten Messzeitfenster liegen für Versuchsbedingung I innerhalb dieser Schranken. 


\subsubsection{Interferometrische Messungen der Zylinderströmung}

Die Dichteauswertung der gemessenen Phasenverschiebung der holographischen Interferometrie beruht, da es sich um ein sichtlinienintegrierendes Verfahren handelt, auf bestimmten Annahmen bezüglich der Strömung (s. Abschnitt 4.1.2). Zu diesen Annahmen gehört, dass die Strömung rotationssymmetrischen oder zweidimensionalen Charakter besitzt, weiterhin darf sich die chemische Zusammensetzung des Gases entlang einer Sichtlinie nicht zu stark ändern. In Abb. $\mathbf{5 . 6}$ sind Phasenverschiebungen entlang der Staustromlinie aufgetragen, welche sich aus künstlichen Interferogrammen ergeben, die mit Hilfe eines Strahlverfolgungsprogramms aus den numerischen Lösungen bestimmt wurden. Es ist deutlich zu erkennen, dass sich die Phasenverschiebung, die ein sichtlinienintegrierendes Verfahren wie die holographische Interferometrie für eine rein zweidimensionale Strömung misst, stark von der einer dreidimensionalen Strömung unterscheidet. Der Anstieg der Dichte und damit auch der Phasenverschiebung ist hinter dem Verdichtungsstoß für die dreidimensionale Strömung nicht so stark ausgeprägt wie für die zweidimensionale Rechnung. Gerade aber dieser Verlauf wird, wie in Abschnitt 2.2 diskutiert, vom Relaxationsverhalten der Strömung bestimmt.

Die echte Anströmung des Zylindermodells im HEG ist konisch, da eine konische Hyperschalldüse verwendet wird. Berücksichtigt man dies, wie in Abb. 5.6 zu sehen, verringert sich zusätzlich noch der Abstand des Verdichtungsstoßes zur Oberfläche des Zylinders. Das Verhältnis von Länge zu Durchmesser des Zylinders ist größer als vier, trotzdem kann die Strömung nicht als zweidimensional angesehen werden. Zu einer Validierung der Relaxationsmodellierung innerhalb der Numerik muss die Zylinderströmung also dreidimensional simuliert werden, um sie mit den Messergebnissen der holographischen Interferometrie zu vergleichen. Das Verhältnisse $f_{\Delta}$ des Stoßabstandes von dreidimensionaler zu zweidimensionaler Rechnung ist in Tab. $\mathbf{5 . 4}$ für die vier nachgerechneten Versuche aufgeführt.
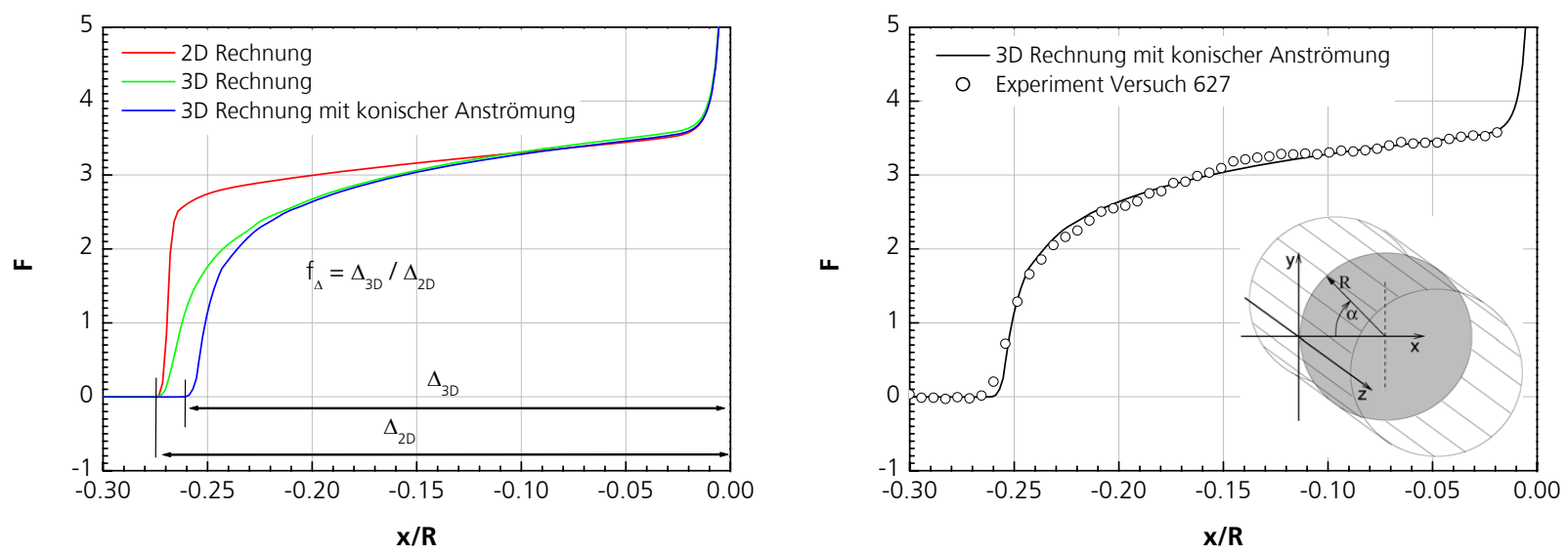

Abbildung 5.6: Einfluss der Dreidimensionalität der Zylinderumströmung. Aufgetragen ist die Phasenverschiebung in der Stoßschicht der Zylinderströmung über dem dimensionslosen Stoßabstand.

In Abb. $\mathbf{5 . 7}$ sind die experimentell gewonnenen Phasenverschiebungen für die Staustromlinie mit den numerischen Ergebnissen zusammen aufgetragen. Die beste Übereinstimmung existiert für den Versuch 627 (Bed. III, Luft). Sowohl der Anstieg als auch der Stoßabstand 


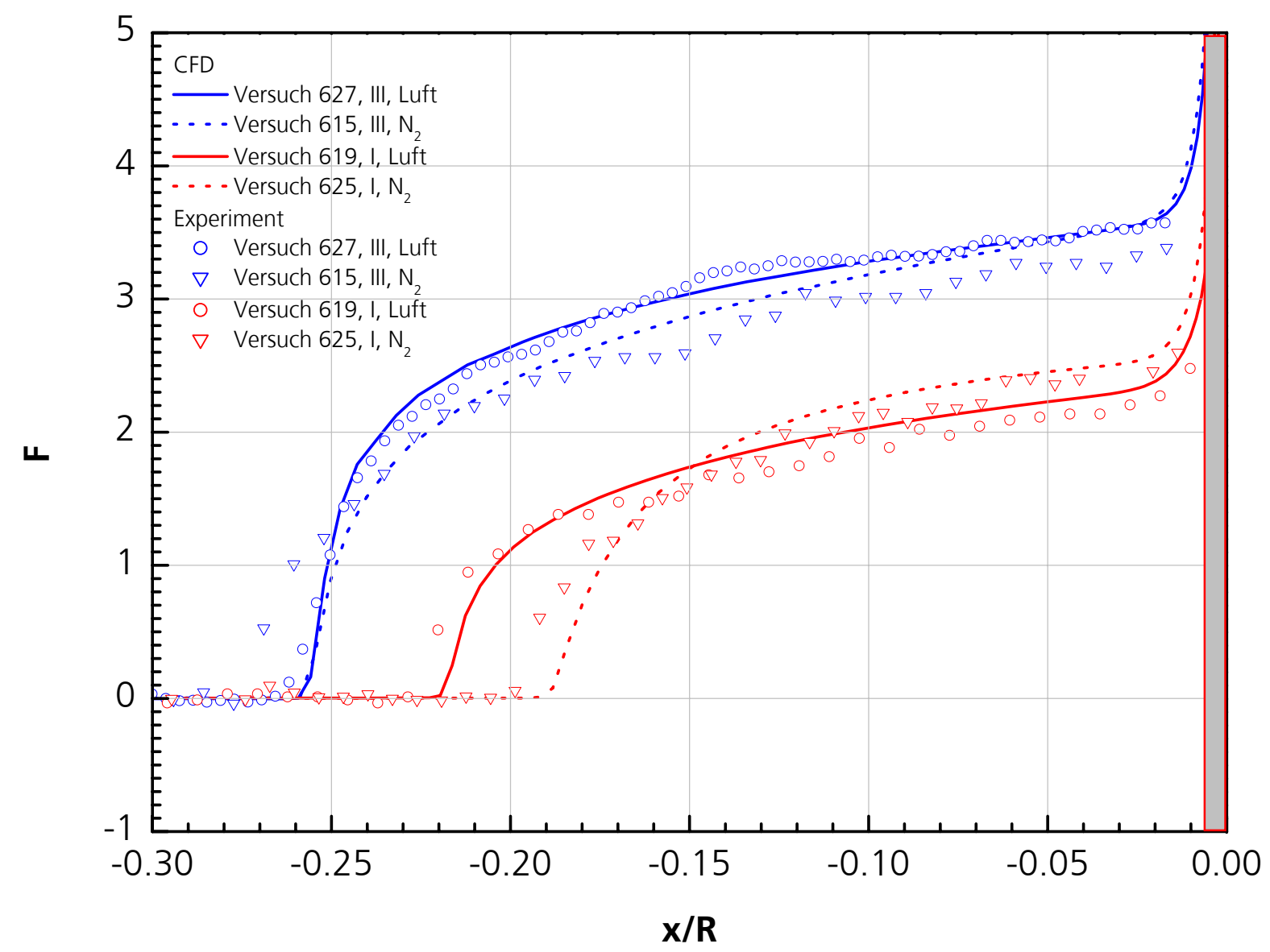

Abbildung 5.7: Vergleich der numerischen und der experimentellen Phasenverschiebung der Zylinderströmung für alle Bedingungen auf der Staustromlinie. Der graue Bereich markiert die optische Grenze, bei der eine Auflösung der Streifen der Interferogramme nicht mehr gewährleistet ist. Phasenverschiebung auf $2 \pi$ normiert.

stimmen sehr gut überein. Für die anderen Bedingungen wird der Stoßabstand zu klein vorhergesagt.

Tab. 5.4 enthält die Zahlenwerte für die Stoßabstände aus den Experimenten und auch aus den dreidimensionalen Rechnungen. Die dreidimensionalen Rechnungen sind alle, wie beim Vergleich der Oberflächenwerte, mit dem Gupta-Modell durchgeführt worden, bis auf die Rechnung für Versuch 615, hier wurde das Reaktionsratenmodell von Park verwendet. Im Vergleich mit den experimentellen Daten beträgt die größte Abweichung zu den numerischen dreidimensionalen Ergebnissen 6.52 \% für den Stoßabstand bei Versuch 615 (Bed. III, $\mathrm{N}_{2}$, Park).

Um den Einfluss der Reaktionsratenmodelle auf den Stoßabstand diskutieren zu können, wird das Verhältnis $\Delta f$ des Stoßabstandes der dreidimensionalen Rechnungen zu dem der zweidimensionalen Rechnungen gebildet. Diese Faktoren sind in Tab. $\mathbf{5 . 5}$ angegeben. Die experimentell ermittelten Stoßabstände werden in Tab. 5.5 mit den jeweiligen Faktoren multipliziert und dann mit den Stoßabständen aus den zweidimensionalen Rechnungen für 


\begin{tabular}{|ccccccc|}
\hline Versuch & Bedingung & Experiment & 3D-CFD & & 2D-CFD & \\
& & $\Delta / \mathbf{R}$ & $\Delta / \mathbf{R}$ & Abweichung & $\Delta / \mathbf{R}$ & $\mathbf{f}_{\Delta}=(\Delta / \mathbf{R})_{\mathbf{3 D}} /(\Delta / \mathbf{R})_{\mathbf{2}}$ \\
\hline 627 & III, Luft & 0.264 & 0.255 & $3.41 \%$ & 0.270 & 1.059 \\
615 & III, N $N_{2}$ & 0.276 & 0.258 & $6.52 \%$ & 0.270 & 1.047 \\
619 & I, Luft & 0.228 & 0.219 & $3.95 \%$ & 0.230 & 1.050 \\
625 & $\mathrm{I}, \mathrm{N}_{2}$ & 0.201 & 0.188 & $6.47 \%$ & 0.200 & 1.064 \\
\hline
\end{tabular}

Tabelle 5.4: Vergleich der experimentellen und der numerischen Stoßabstände für die dreidimensionalen Rechnungen.

\begin{tabular}{|cccccccccc|}
\hline Versuch & Bedingung & Exp. & Exp. $^{*} \mathbf{f} \Delta$ & Gupta & \multicolumn{3}{c|}{ Dunn und Kang } & \multicolumn{2}{c|}{ Park } \\
& & $\Delta / \mathbf{R}$ & $\Delta / \mathbf{R}$ & $\Delta / \mathbf{R}$ & Abw. & $\Delta / \mathbf{R}$ & Abw. & $\Delta / \mathbf{R}$ & Abw. \\
\hline 627 & III, Luft & 0.264 & 0.280 & 0.270 & $3.41 \%$ & 0.250 & $10.56 \%$ & 0.220 & $21.30 \%$ \\
615 & III, N & 0.276 & 0.289 & 0.250 & $13.45 \%$ & 0.240 & $16.91 \%$ & 0.270 & $6.52 \%$ \\
619 & I, Luft & 0.228 & 0.239 & 0.230 & $3.95 \%$ & 0.220 & $8.12 \%$ & 0.210 & $12.30 \%$ \\
625 & $\mathrm{I}_{2} \mathrm{~N}_{2}$ & 0.201 & 0.214 & 0.200 & $6.47 \%$ & 0.190 & $11.14 \%$ & 0.240 & $-12.24 \%$ \\
\hline
\end{tabular}

Tabelle 5.5: Vergleich der experimentellen und der numerischen Stoßabstände für die zweidimensionalen Rechnungen.

verschiedene Modelle verglichen. Die größte Abweichung beträgt 21.30 \%. In Abb. $\mathbf{5 . 9}$ sind die Abweichungen für die verschiedenen Reaktionsratenmodelle graphisch über einer mittleren Damköhlerzahl auf der Staustromlinie aufgetragen. Diese Damköhlerzahl (s. 2.2) ist als das Verhältnis des Energieaustausches durch chemische Reaktionen zum Gesamtenergieeintrag der Anströmung definiert:

$$
D a^{m i t t e l}=\frac{\Delta \sum_{S} \dot{w}_{s} h_{s}}{\rho_{\infty} u_{\infty}^{3}} .
$$

Hierbei sind $\rho_{\infty}$ und $u_{\infty}$ die Dichte und Geschwindigkeit der freien Anströmung, $\Delta$ ist der Stoßabstand, $\dot{w}_{S}$ ist die Produktionsrate der Spezies $S$ und $h_{S}$ ist die spezifische Enthalpie der Spezies $S$.

In Abb. $\mathbf{5 . 8}$ sind die numerischen Stoßabstände für die zweidimensionalen Rechnungen der Zylinderumströmung für die verschiedenen HEG-Versuche und die verwendeten Reaktionsratenmodelle über der Damköhlerzahl aufgetragen. Es ist zu erkennen, dass sich der Stoßabstand mit höherer mittlerer Damköhlerzahl verkleinert. Dies ist zu erwarten, da eine höhere mittlere Damköhlerzahl eine höhere chemische Aktivität bedeutet, die die Strömung schneller ins Gleichgewicht bringt: der Stoßabstand muss kleiner werden. Trägt man nun aber wie in Abb. 5.9 die Abweichungen der numerischen Stoßabstände zu den experimentellen auf, sagt die Modellierung nach Dunn und Kang mit zunehmender mittlerer Damköhlerzahl in der Stoßschicht den Stoßabstand immer besser vorher. Bei den beiden anderen Modellen ist so ein Trend nicht zu erkennen.

In den Abbildungen $\mathbf{5 . 1 0}$ (Bedingung I) und $\mathbf{5 . 1 1}$ (Bedingung III) sind die Vergleiche zwischen den numerisch gebildeten und den experimentell ermittelten Phasenverschiebungen dargestellt. In diesen Auftragungen wird deutlich, dass die Grenzschicht direkt an der Wand nicht aufgelöst werden kann. Für die Versuchsbedingung III ist die Übereinstimmung in der Stoßschicht zwischen Rechnung und Experiment generell besser als für die Bedingung I. 


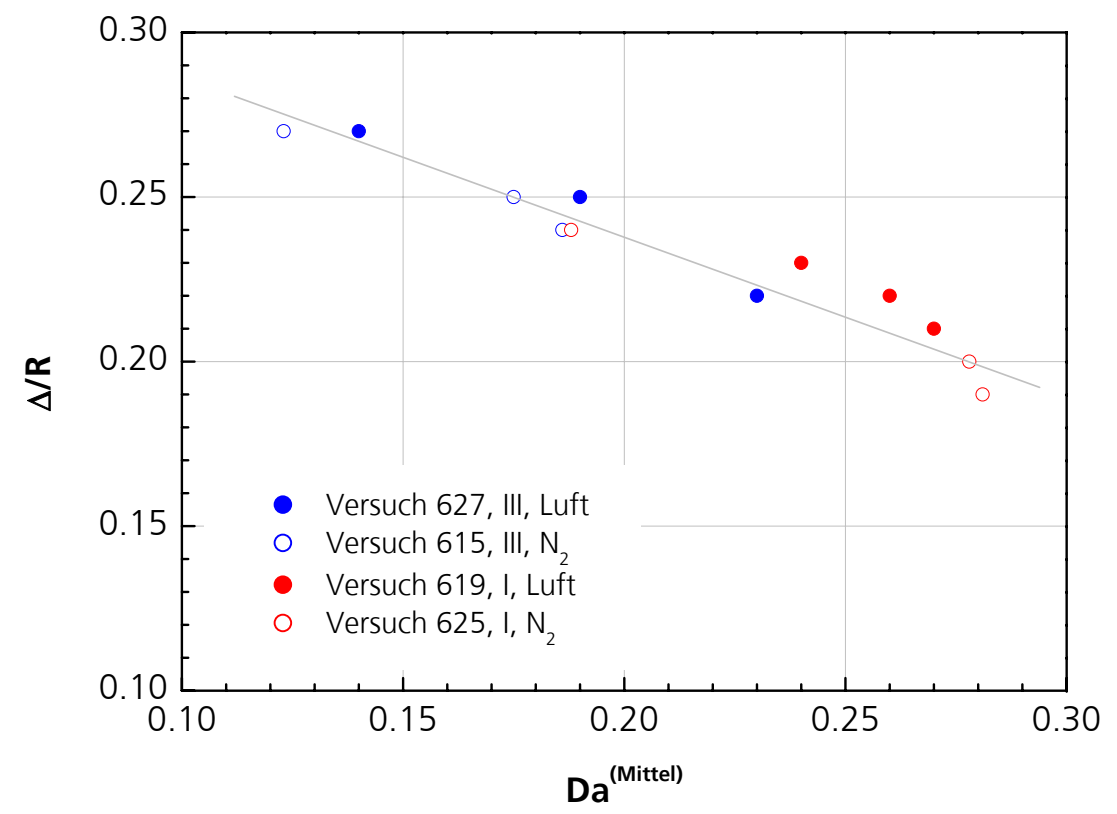

Abbildung 5.8: Variation der Stoßabstände mit dem verwendeten Reaktionsratenmodell.

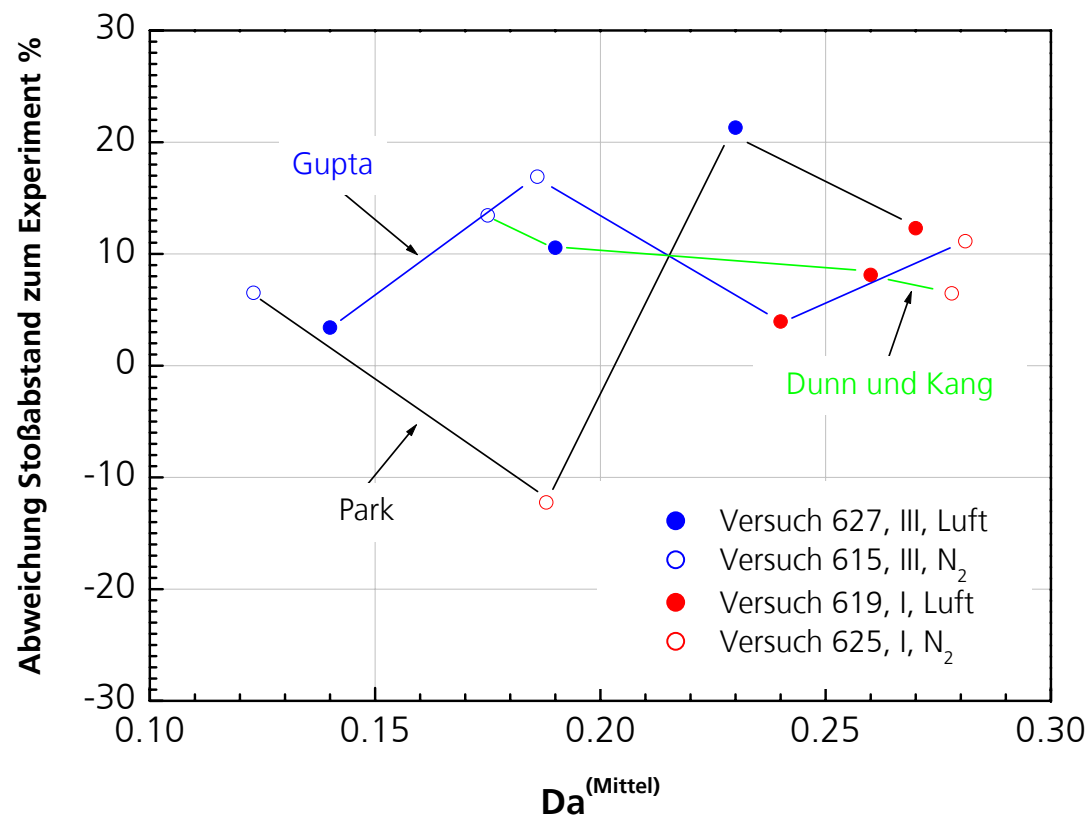

Abbildung 5.9: Abweichung der numerische Stoßabstände vom Experiment in Abhängigkeit von der mittleren Damköhlerzahl. 

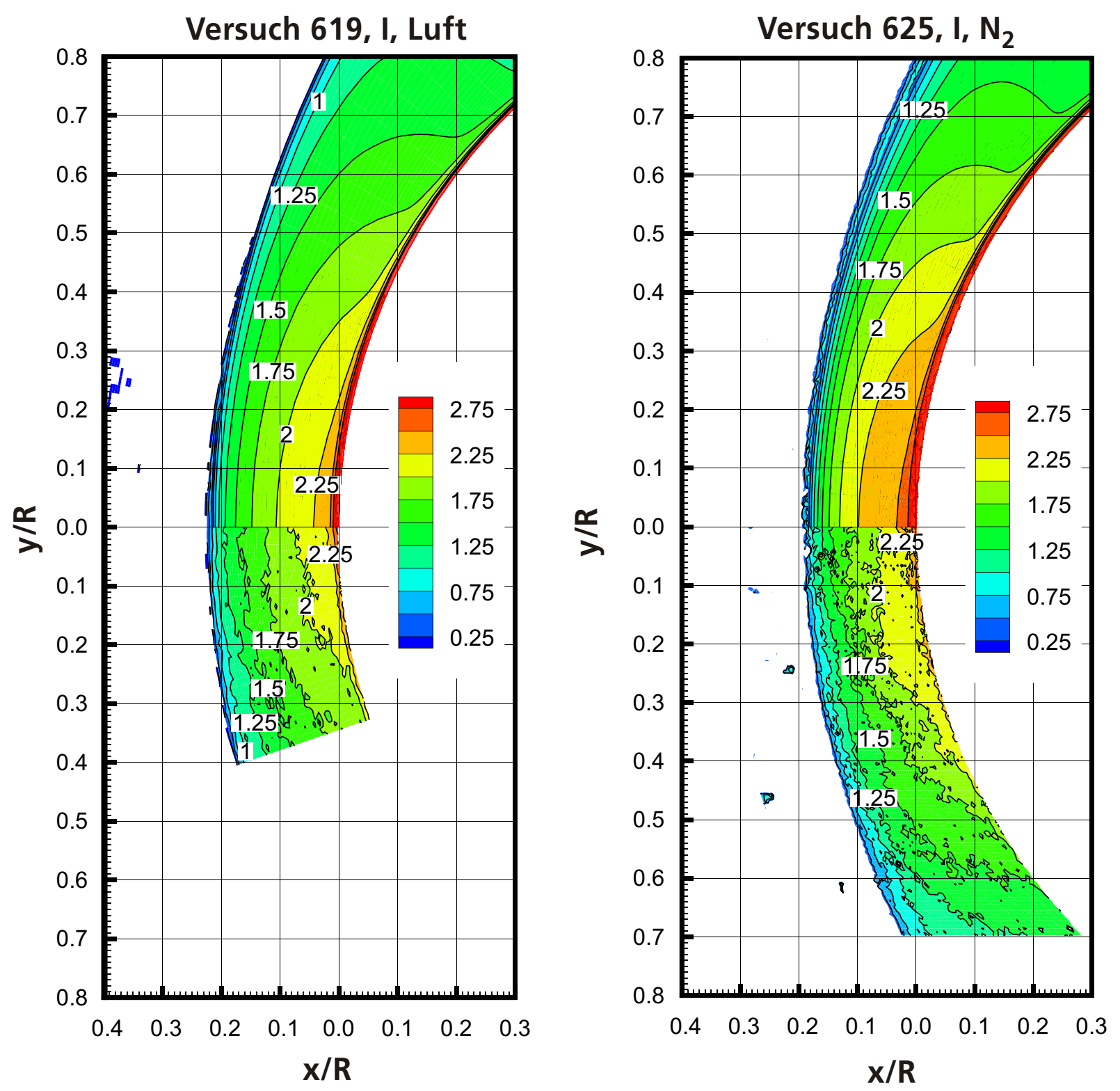

Abbildung 5.10: Vergleich der numerischen und der experimentellen Phasenverschiebung der Zylinderumströmung für Bedingung I bei Luft (links) und Stickstoff (rechts). Phasenverschiebung auf $2 \pi$ normiert. 

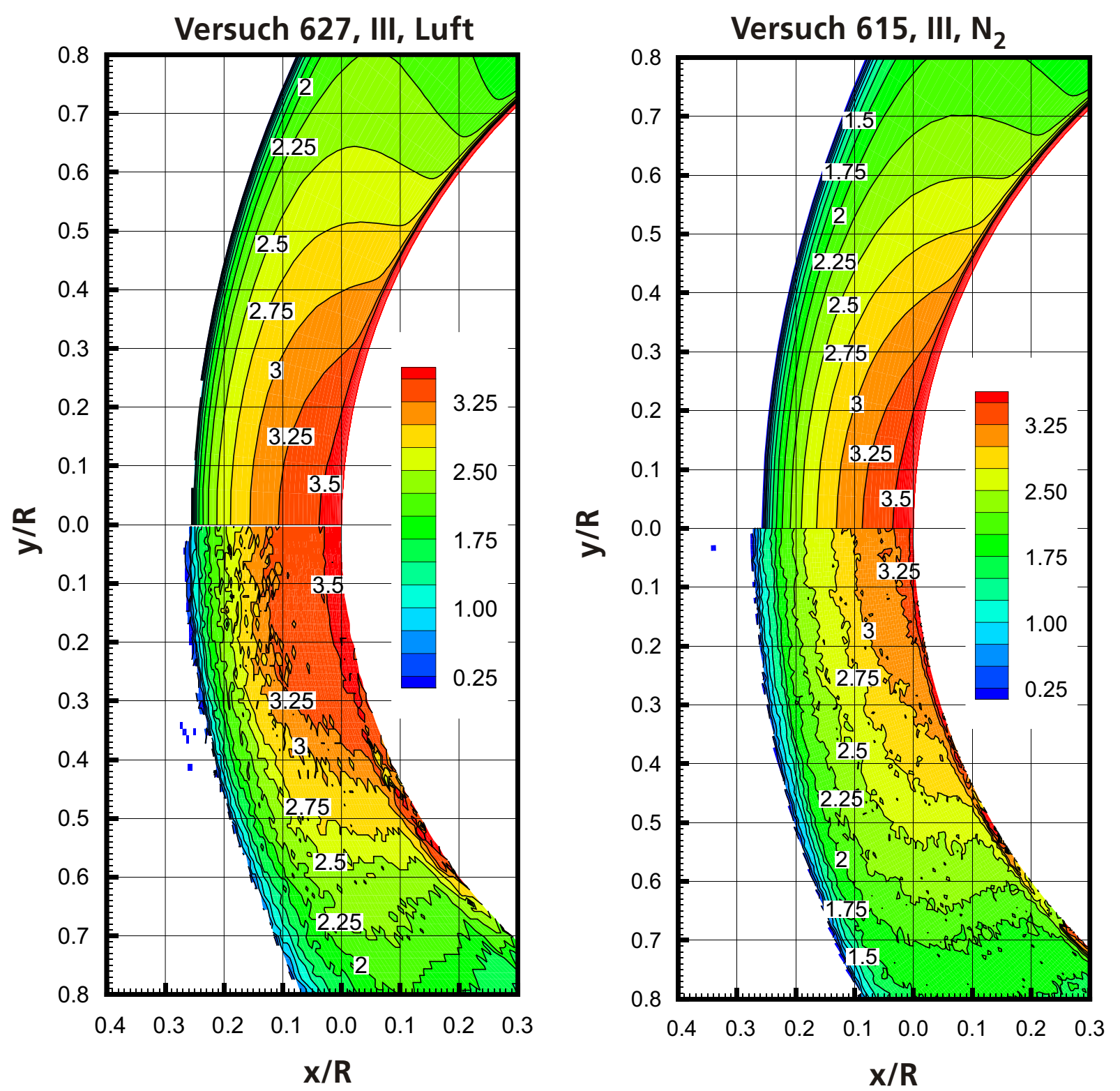

Abbildung 5.11: Vergleich der numerischen und der experimentellen Phasenverschiebung der Zylinderumströmung für Bedingung III bei Luft (links) und Stickstoff (rechts). Phasenverschiebung auf $2 \pi$ normiert. 


\subsection{X-38-Modell}

\subsubsection{Durchgeführte Versuche}

Die im HEG durchgeführten Experimente mit dem X-38-Modell sind in Tab. $\mathbf{5 . 6}$ aufgelistet. Insgesamt wurden 16 Versuche mit einem Anstellwinkel des Modells von $\alpha=40^{\circ}$ durchgeführt.

Bei den ersten acht Versuchen mit der HEG-Bedingung III wurde sowohl der Klappenwinkel $\delta$ als auch der Schiebewinkel $\beta$ des Modells variiert. Bei HEG-Bedingung I wurden ebenfalls acht Versuche durchgeführt; hier wurde nur der Klappenwinkel geändert. In allen Experimenten waren die Klappen symmetrisch angestellt. Da bei den Messungen die Reproduzierbarkeit der Ergebnisse von Versuch zu Versuch im Vordergrund stand, werden die Ergebnisse für gleiche Bedingungen entdimensionalisiert und durch Mittelung zusammengefasst. Die Standardabweichung in den Auftragungen gibt die Schwankung des SensorMesswertes von Versuch zu Versuch an. Bei HEG-Bedingung III mit $\delta=20^{\circ}$ sind die Daten über vier Versuche gemittelt, bei $\delta=30^{\circ}$ über 2 Versuche. Für die HEG-Bedingung I sind fünf Versuche für $\delta=20^{\circ}$ gemittelt worden und für $\delta=30^{\circ}$ drei Versuche.

\begin{tabular}{|c|c|c|c|c|c|c|c|c|c|}
\hline Versuch & Bedingung & $\alpha$ & $\delta$ & $\beta$ & Versuch & Bedingung & $\alpha$ & $\delta$ & $\beta$ \\
\hline 517 & III & $40^{\circ}$ & $20^{\circ}$ & $0^{\circ}$ & 551 & I & $40^{\circ}$ & $20^{\circ}$ & $0^{\circ}$ \\
\hline 544 & III & $40^{\circ}$ & $20^{\circ}$ & $0^{\circ}$ & 552 & I & $40^{\circ}$ & $20^{\circ}$ & $0^{\circ}$ \\
\hline 545 & III & $40^{\circ}$ & $20^{\circ}$ & $0^{\circ}$ & 553 & I & $40^{\circ}$ & $20^{\circ}$ & $0^{\circ}$ \\
\hline 546 & III & $40^{\circ}$ & $20^{\circ}$ & $0^{\circ}$ & 554 & I & $40^{\circ}$ & $20^{\circ}$ & $0^{\circ}$ \\
\hline 556 & III & $40^{\circ}$ & $20^{\circ}$ & $5^{\circ}$ & 555 & I & $40^{\circ}$ & $20^{\circ}$ & $0^{\circ}$ \\
\hline 557 & III & $40^{\circ}$ & $20^{\circ}$ & $5^{\circ}$ & 560 & I & $40^{\circ}$ & $30^{\circ}$ & $0^{\circ}$ \\
\hline 558 & III & $40^{\circ}$ & $30^{\circ}$ & $0^{\circ}$ & 561 & I & $40^{\circ}$ & $30^{\circ}$ & $0^{\circ}$ \\
\hline 559 & III & $40^{\circ}$ & $30^{\circ}$ & $0^{\circ}$ & 562 & I & $40^{\circ}$ & $30^{\circ}$ & $0^{\circ}$ \\
\hline
\end{tabular}

Tabelle 5.6: Übersicht der durchgeführten Versuche mit dem X-38-Modell.

\subsubsection{Druckmessungen}

Da das Modell des X-38 keine Druckanbohrung für den Staupunkt hat, sind die Druckmessungen an der Modelloberfläche mit dem Staudruck, der auf dem Pitotrohr der permanenten Sonde (s. Abschnitt 3.3.5) bei jedem Versuch gemessen wurde, normiert. Diese normierten Druckwerte des Einzelversuches wurden für den Vergleich mit den numerischen Daten für alle Versuche gleicher Klappenanstellung gemittelt.

In Abb. 5.12 auf der linken Seite sind die dimensionslosen Druckverläufe für die HEGVersuchsbedingung I bei $\delta=20^{\circ}$ Klappenausschlag dargestellt. Hier sind die beiden Gruppen PRESSBO und PRESS20 für den Messmittelschnitt und den Schnitt über die Klappen zusammen mit den numerischen Lösungen aufgetragen. Die Vorkörpergruppe PRESSBO besteht aus 4 Sensoren, die Gruppe PRESS20 aus 11 Sensoren in einem Schnitt, der fast parallel zur Symmetrielinie liegt. Die Druckverteilung auf dem Vorkörper, die Expansion vor der Scharnierlinie und der Druckanstieg auf der Klappe sind klar aufgelöst. Generell ist die quantitative Übereinstimmung zwischen den Messergebnissen und den numerischen Ergebnissen 

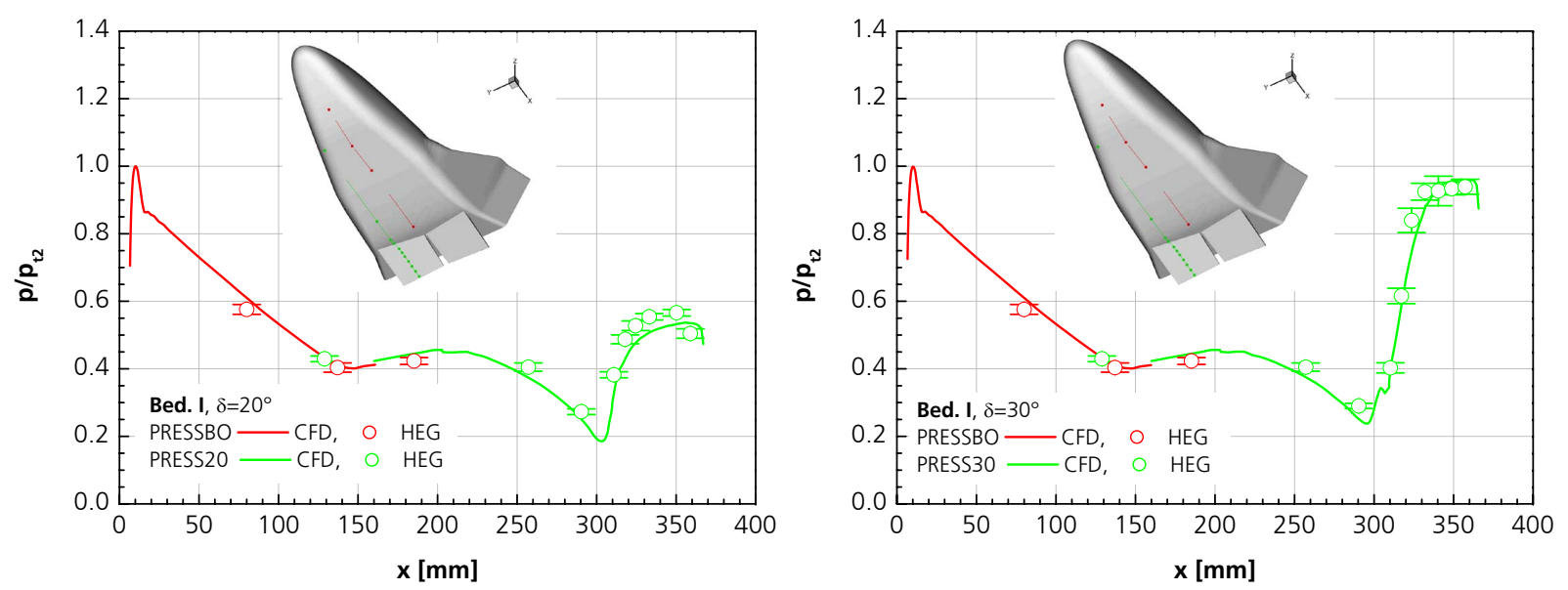

Abbildung 5.12: Druckmessung X-38-Modell (Bed. I, $\delta=20^{\circ} / 30^{\circ}$ ).

gut. Die mittlere Abweichung liegt innerhalb von 2\%. Das Druckniveau der Rechnung für die Klappe mit $\delta=20^{\circ}$ ist ungefähr $10 \%$ niedriger als die Messung. In Abb. 5.12 rechts ist die normierte Druckverteilung für den Klappenausschlag von $\delta=30^{\circ}$ aufgetragen (Gruppe PRESSBO, PRESS30). Insgesamt ist die Übereinstimmung gut, und die Wiederholbarkeit der Messungen liegt innerhalb von 3\%. Der Vergleich zwischen der Rechnung und der Messung fällt für die $30^{\circ}$-Klappe besser aus als der Vergleich für die $20^{\circ}$-Klappe, die Abweichung zwischen Messung und Rechnung ist hier kleiner als 10\%.

Für Bedingung III und $\delta=20^{\circ}$ Klappenausschlag ist die dimensionslose Druckverteilung in Abb. 5.13 wiedergegeben (Gruppe PRESSBO, PRESS20). Zusätzlich finden sich die Ergebnisse für die Messungen mit einem Schiebewinkel von $\beta=5^{\circ}$. Quantitativ ist die Wiederholbarkeit und auch der Vergleich zwischen Messungen und Rechnung ähnlich wie bei Bedingung I. Der größte Einfluss, der durch die $5^{\circ}$ Schiebewinkel des Modells entsteht, ist die Verschiebung des Druckmaximums in Strömungsrichtung. Die dimensionslose Druckverteilung für Bedingung III und einen Klappenausschlag von $30^{\circ}$ ist ebenfalls in Abb. 5.13 aufgetragen (Gruppe PRESSBO, PRESS30). Auch hier ist der Vergleich zwischen Rechnung und Messung gut. Nach dem Wiederanlegen der Strömung beträgt die größte Abweichung $10 \%$. Zusammenfassend lässt sich sagen, dass im Vergleich der numerischen Druckverteilungen mit den gemessenen die numerischen Vorhersagen für $20^{\circ}$ Klappenauschlag zu niedrig sind, während die Rechnungen für $30^{\circ}$ Klappenausschlag insgesamt zu hoch liegen; dies aber im gleichen Maße bei beiden Versuchsbedingungen (Bed. I und III).

\subsubsection{Wärmestromdichtemessungen}

In diesem Abschnitt werden die Wärmestromdichtemessungen auf der Oberfläche des X38-Modells diskutiert. Die Wärmestromdichtemessungen wurden mit dem Wert des Staupunktsensors entdimensionalisiert. Dieses Vorgehen wurde sowohl für die numerischen Ergebnisse als auch für die Messungen angewandt. Nach Versuch 546 wurde das Staupunktthermoelement durch einen Partikeleinschlag zerstört und war nicht mehr zu reaktivieren. Deshalb wurde der Messwert der permanenten Sonde (s. Abschnitt 3.3.5) für die Wärme- 

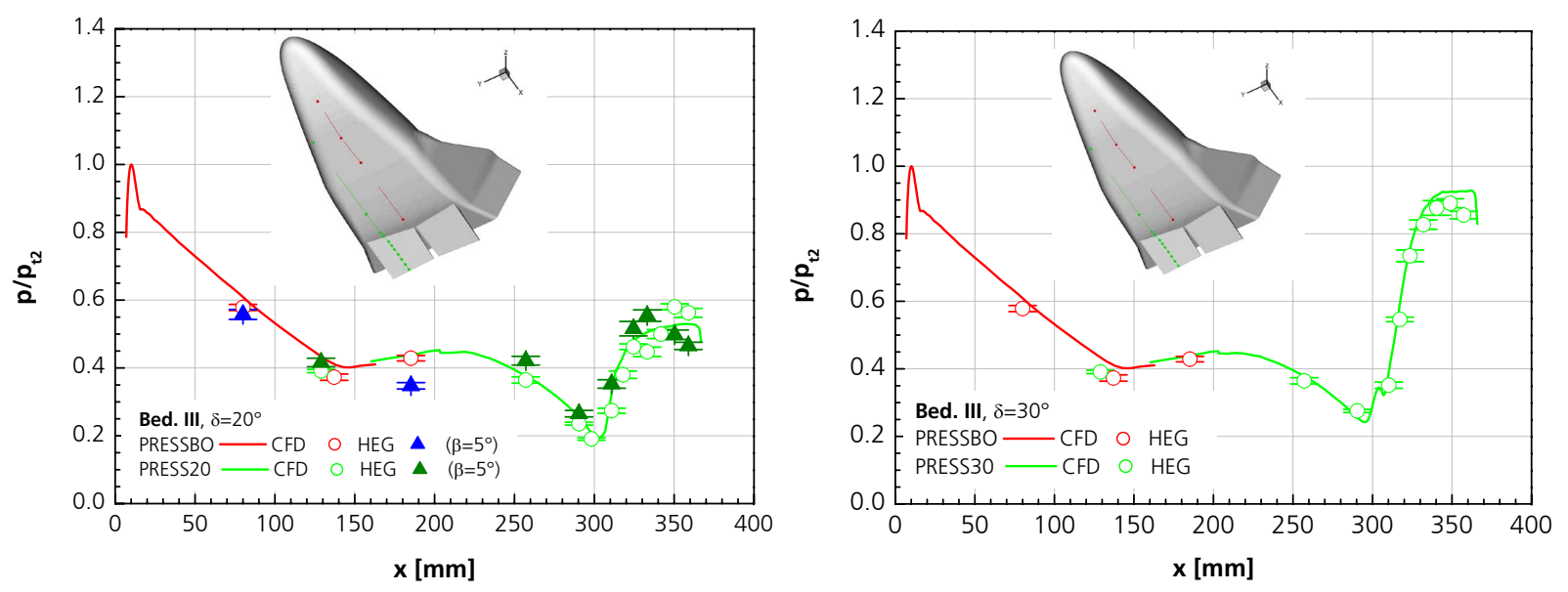

Abbildung 5.13: Druckmessung X-38-Modell (Bed. III, $\delta=20^{\circ} / 30^{\circ}, \beta=5^{\circ}$ ).

stromdichte im Staupunkt der Halbkugel für die Normierungen benutzt. Der Messwert der permanenten Sonde wurde über das Verhältnis (Radius permanente Sonde/Nasenradius des Modells) folgendermaßen skaliert:

$$
\dot{q}_{r e f}=\dot{q}_{t 2} \sqrt{\frac{r_{p p}}{r_{r e f-M o d e l l}}} .
$$

Da das X-38-Modell keine sphärische Nasenform besitzt (die Nasenregion besteht aus zwei Ellipsen), wurde der Referenz-Radius von $1 \mathrm{ft}$ für das X-38-V201-Vehikel ( s. Muratore u. a. (2000)) gewählt. Der Radius errechnet sich dann zu $r_{\text {ref-Modell }}=304.8 \mathrm{~mm} / 24=12.7 \mathrm{~mm}$.

Um diese Vorgehensweise zu überprüfen, wurden die mit Gl. 5.1 bestimmten Staupunktwärmestromdichten mit den bis Versuch 546 vorhandenen Werten des Staupunktsensors verglichen. Durchschnittlich war die Abweichung zwischen den rekonstruierten Werten und den gemessenen Werten 4\%. Dieser Wert liegt deutlich unter dem angenommenen maximalen Fehler von $\pm 15 \%$ für die Wärmestromdichtemessungen. Die in den folgen Abschnitten diskutierten Ergebnisse bilden, wie bei den Druckmessungen auch, eine Mittelung über alle durchgeführten Versuche. Die Standardabweichung ist mit in den Abbildungen eingetragen und ist wie auch bei den Druckmessungen ein Maß für die Schwankungen der Resultate von Versuch zu Versuch.

Einer besseren Übersicht wegen wurde die Gruppe BOSY in zwei Abbildungen aufgetragen. In Abb. 5.14 sind die Ergebnisse der Wärmestromdichtemessungen und Rechnungen für die Symmetrielinie auf dem Vorkörper aufgetragen. Die Ergebnisse für die Versuchsbedingungen I und III sind hier zusammengefasst. Die Standardabweichung von Versuch zu Versuch beträgt maximal 10\%. Die größte Abweichung findet sich im Nasenbereich $(6 \mathrm{~mm}<x<30 \mathrm{~mm})$. Für die Sensorpositionen $x>90 \mathrm{~mm}$ ist die Übereinstimmung mit der Rechnung gut. Die mittlere Abweichung über alle Sensoren auf dem Vorkörper ergibt sich zu $15 \%$. Für fast alle Messpunkte bei den Versuchsbedingungen I und III (mit oder ohne Schiebewinkel $\beta$ ) zeigt sich eine deutliche Überlappung der Standardabweichungen, so dass keine Unterscheidung der Verläufe möglich ist. 
In Abb. 5.14 sind auch die gemessenen Wärmestromdichten der Sensoren im Klappenschacht auf der Symmetrielinie aufgetragen. Die sehr kleinen Messsignale der Thermoelemente im Klappenschacht führen zu einer höheren Standardabweichung als auf dem Vorkörper. Die Schwankungen der Messwerte von Versuch zu Versuch liegen hier im Bereich von $30 \%$. Sowohl für die Klappe mit $\delta=20^{\circ}$ Anstellwinkel als auch für die mit $\delta=30^{\circ} \mathrm{An}$ stellwinkel folgen die ersten beiden Sensoren dem von der Numerik vorhergesagten Trend. Aber für die Sensoren L08 und L09 (dritter und vierter Sensor im Klappenschacht) ist der gemessene Wert deutlich niedriger als die numerischen Ergebnisse. Diese starke Abweichung lässt sich auf einen Geometrieunterschied zwischen dem realen Windkanalmodell und dem numerischen Gitter zurückführen (Martinez Schramm u. a. (2001)). Die Messungen zeigen, dass die normierte Wärmestromdichte im Mittel für die Bedingung III höher liegt als für die Bedingung I. Auf der letzten Sensorposition ergeben die Messungen für die $20^{\circ}$-Klappe einen höheren Wärmestrom als für die $30^{\circ}$-Klappe. Dieses liegt an der um die Klappen expandierenden Strömung (vergl. Abb. 5.16). Bei kleinerem Klappenwinkel bewirkt die Wechselwirkung dieses heißen Gases mit der Oberfläche eine höhere Wärmestromdichte als für den größeren Anstellwinkel. Dieses Ergebnis wird auch von der CFD gestützt, allerdings ist dieser Effekt in der CFD durch den geometrischen Unterschied zwischen Rechennetz und Windkanalmodell für die Klappenkastenform nicht so stark ausgeprägt. Die gemessene Wärmestromdichte im Klappenschacht an den Sensorpositionen L04, L08 und L09 (zweiter, dritter und vierter Sensor) ist bei $\beta=5^{\circ}$ Schiebewinkel um $50 \%$ niedriger als bei den Messungen ohne Schiebewinkel.

Die normierten Wärmestromdichtemessungen für die Gruppen FLAP20 und FLAP30 bei der Versuchsbedingung III sind in Abb. $\mathbf{5 . 1 5}$ rechts aufgetragen. Diese Gruppe beinhaltet neben den 9 Sensoren auf der Klappe auch 4 Sensoren auf dem Vorkörper. Der Schnitt, den diese Sensoren bilden, liegt fast parallel zur Symmetrielinie. Außer an der ersten Sensorposition, wo die gemessene Wärmestromdichte deutlich höher als die berechnete ist, ist die Übereinstimmung zwischen der Rechnung und der Messung gut. Die Abweichungen liegen bei maximal $15 \%$. Nach dem Wiederanlegen der Strömung auf der $20^{\circ}$-Klappe ist die gemessene Wärmestromdichte maximal 5\% höher als der berechnete. Im Wiederanle-
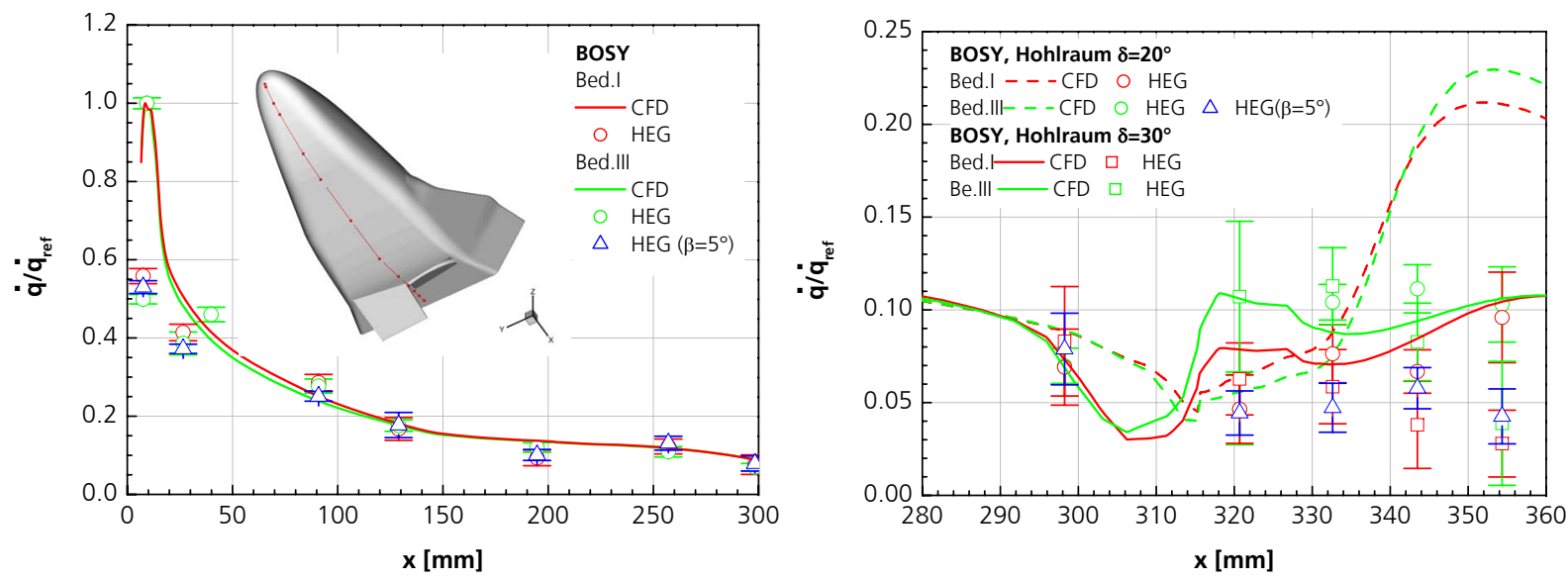

Abbildung 5.14: Wärmestromdichtemessung auf dem Vorkörper und im Klappenschacht. 

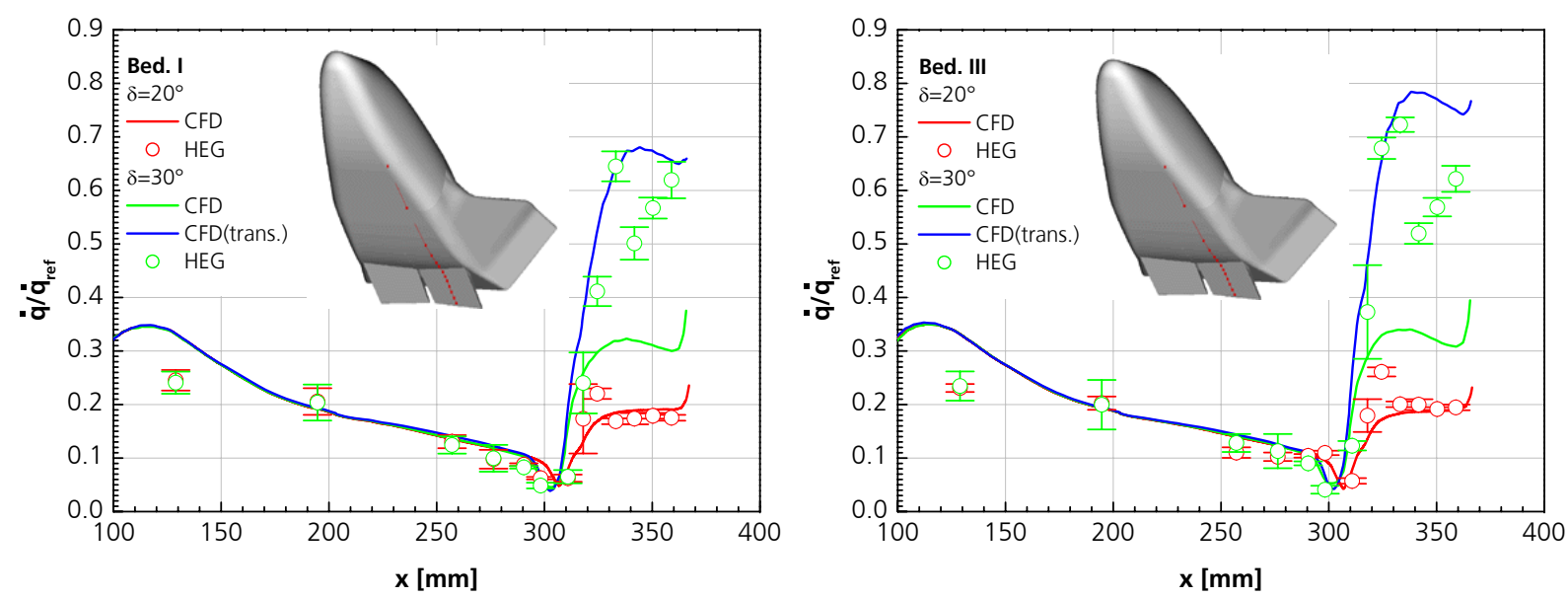

Abbildung 5.15: Wärmestromdichtemessung X-38-Modell (Bed. IIIII, $\left.\delta=20^{\circ} / 30^{\circ}\right)$.

gebereich der Strömung zeigen die Messungen ein deutliches Maximum, das die Rechnung nicht wiedergibt. Dieses Maximum liegt 40\% höher als die berechnete Wärmestromdichte.

Es ist auch deutlich zu erkennen, dass die Standardabweichung der Messwerte im Ablösegebiet der Strömung größer ist als auf dem Rest des Körpers. Dieses Verhalten ist in allen Messungen zu beobachten. In Abb. 5.15 links sind die Wärmestromdichten für die $20^{\circ}$ Klappe und die $30^{\circ}$-Klappe bei Versuchsbedingung I dargestellt. Diese Ergebnisse zeigen das gleiche Verhalten der Strömung wie bei Bedingung III. Die durchschnittliche Abweichung zwischen Messung und Rechnung liegt bei $10 \%$.

Die beiden Rechenergebnisse, die in Abb. $\mathbf{5 . 1 6}$ dargestellt sind, stammen aus zwei unterschiedlichen Rechnungen mit symmetrisch angestellten Klappen. Hier wird deutlich, dass im Vergleich zur $20^{\circ}$-Klappe die Strömung bei einer Umlenkung über die $30^{\circ}$-Klappe eher ablöst und sich der Bereich abgelöster Strömung wie zu erwarten vergrößert. Diese Details sind von den Messungen, die in Abb. 5.15 zu sehen sind, aufgelöst. Weiterhin wird deutlich, dass beim Vergleich der Messung mit der Rechnung für die $30^{\circ}$-Klappe die gemessene Wärmestromdichte ungefähr zwei mal so hoch ist wie die von der laminaren Rechnung

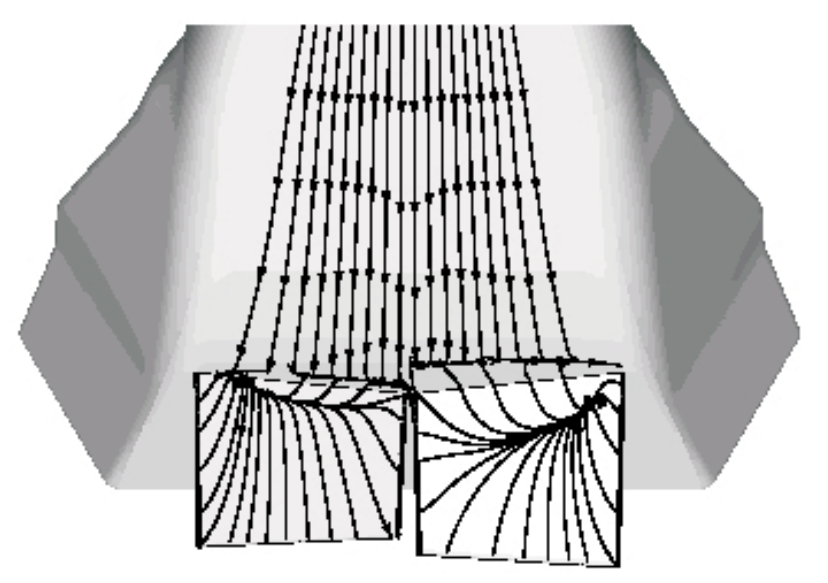

Abbildung 5.16: Numerisches Stromlinienbild (Bedingung III mit $\delta=30^{\circ}$ (rechts) und $\delta=20^{\circ}$ (links). 

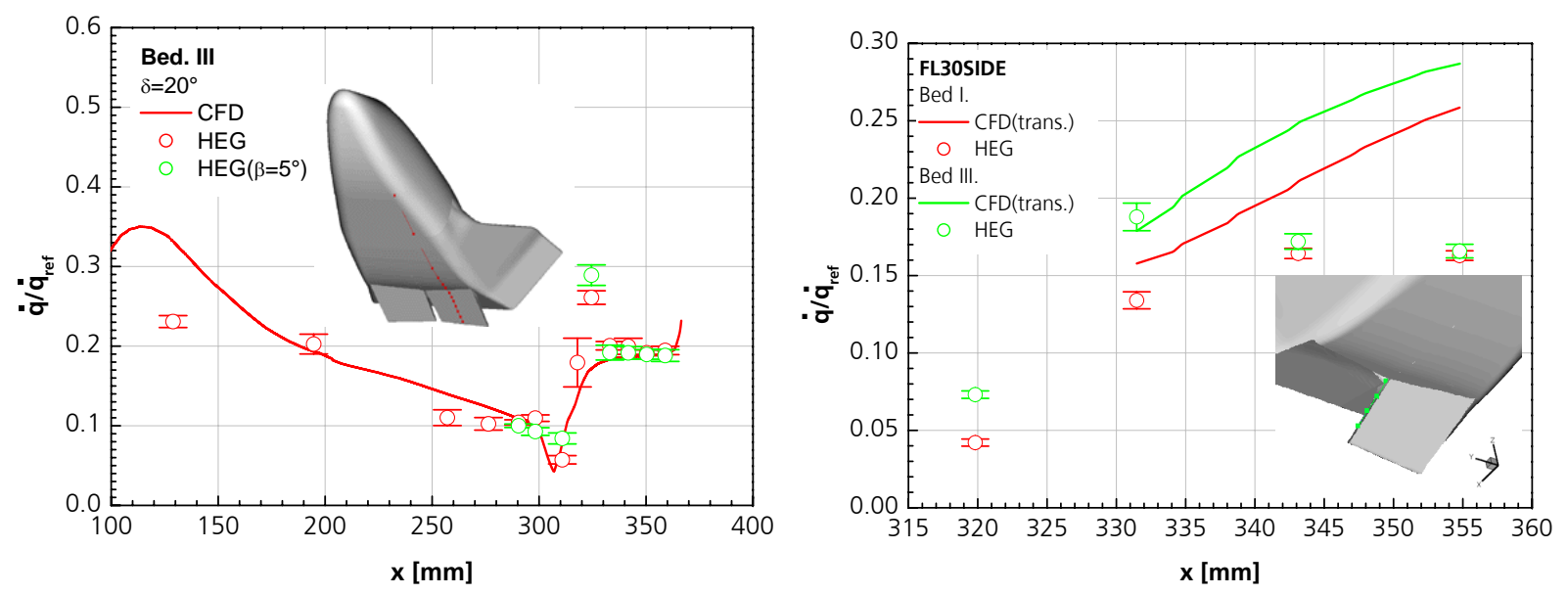

Abbildung 5.17: Wärmestromdichtemessung X-38-Modell (Bed. I,III, $\delta=20^{\circ} / 30^{\circ}, \beta=5^{\circ}$ ).

vorhergesagte Wärmestromdichte. Aus diesem Grund wurde eine transitionelle Rechnung (Hannemann u. a. (2001)) durchgeführt. In dieser Rechnung wurde die Annahme getroffen, dass der Umschlag für den Übergang von einer laminaren zu einer turbulenten Strömung entlang der Scharnierlinie der Klappen stattfindet. Dieses Ergebnis ist ebenfalls in Abb. 5.15 mit aufgetragen. Diese Rechnung reproduziert in etwa den Wärmestromdichtewert beim Wiederanlegen der Strömung, zeigt aber nicht den Abfall und Anstieg der Wärmestromdichte auf den Klappen. Dieses Ergebnis, nach dem die Messungen für die $20^{\circ}$-Klappe zu einer laminaren Rechnung, die Messungen der 30-Klappe eher zu einer transitionellen Rechnung passen, ist bemerkenswert, da ausgehend von der Reynoldszahl der freien Anströmung beider HEG-Bedingungen die Umströmung des X-38-Modells voll laminar sein sollte. Eine Erklärung hierfür wäre, dass Störungen aus der Anströmung in der Ablöseblase angefacht werden und die Strömung umschlägt. Da die abgelöste Region bei der $30^{\circ}$ Klappe größer ist als bei der $20^{\circ}$-Klappe, könnte dieser Effekt hier sehr viel ausgeprägter sein.

Die Messungen der Wärmestromdichte mit $\beta=5^{\circ}$ Schiebewinkel und $\delta=20^{\circ}$ Klappenwinkel bei Versuchsbedingung III wurden ohne die ersten vier Sensoren der Untergruppe FLAP20 durchgeführt. Der Vergleich der Wärmestromdichten mit und ohne Schiebewinkel ist in Abb. 5.17 links dargestellt. Vor dem Ablösen der Strömung ist die Wärmestromdichte niedriger, im Ablösegebiet und beim Wiederanlegen der Strömung höher als für die Messung ohne Schiebewinkel. Das Maximum beim Wiederanlegen der Strömung ist $11 \%$ höher im Vergleich zu der Messung ohne Schiebewinkel. Stromab des Ablösegebiets lässt sich anhand der Messungen kein Unterschied zwischen den Messungen mit und ohne Schiebewinkel erkennen.

Die Wärmestromdichten, die für die vier Sensoren auf der Innenseite der $30^{\circ}$-Klappe bei beiden Bedingungen gemessen wurden, sind in Abb. 5.17 rechts dargestellt. Durch die Unterschiede zwischen Rechennetz und Versuchsmodell (s. (Martinez Schramm u. a. (2001)) ist es nicht sinnvoll, die Messergebnisse für den Sensor FS4 (Abb. 5.17 ganz links) darzustellen. Im Rechennetz ist der Schacht, in den die Klappen einfahren, etwas anders aufgebaut, da das Windkanalmodell hier nicht exakt dem Original gleicht. Der Sensor FS4 liegt im Re- 

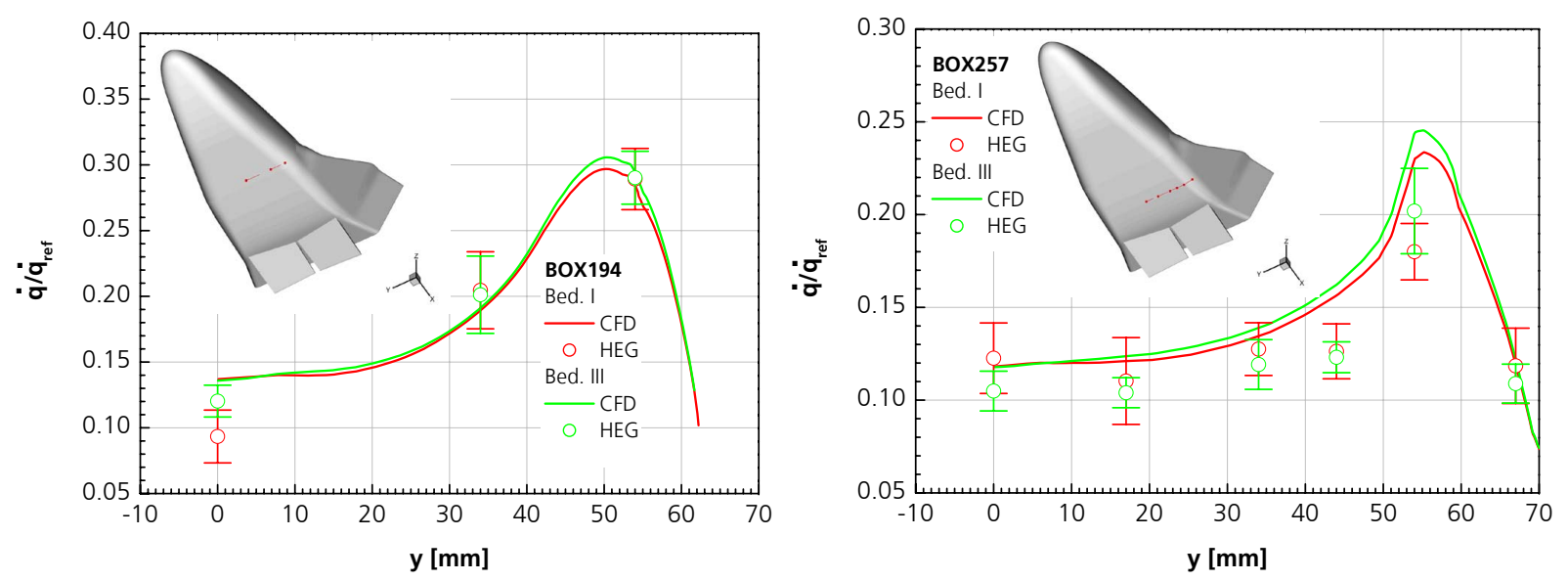

Abbildung 5.18: Wärmestromdichtemessung X-38-Modell (Bed. I,III, Spannw.).

chennetz innerhalb des Körpers. Die in Abb. 5.17 dargestellten numerischen Ergebnisse sind aus den transitionellen Rechnungen entnommen. An den beiden ersten Sensorpositionen steigt die gemessene Wärmestromdichte an. Im Gegensatz zu den numerischen Ergebnissen, die eine kontinuierlich ansteigende Wärmestromdichte zeigen, nehmen die Messungen einen konstanten Wert entlang der Klappeninnenseite an. Die Rechnungen zeigen bei Versuchsbedingung III für die Wärmestromdichte einen konstant höheren Verlauf als für die Versuchsbedingung I. Dieses Verhalten zeigen die beiden ersten Sensoren ebenfalls. Die beiden hinteren Sensoren zeigen allerdings für beide Versuchsbedingungen die gleiche Wärmestromdichte.

In Abb. 5.18 sind die normierten Wärmestromdichten für die Gruppen BOX194 und BOX257 dargestellt. Sie liegen in Spannweitenrichtung auf dem Vorkörper bei $x=194 \mathrm{~mm}$ und $x=257 \mathrm{~mm}$. Generell zeigt sich eine gute Übereinstimmung zwischen den numerischen Vorhersagen und den Messungen. Für die beiden Schnitte ergibt sich ein Unterschied von 15\%, die Standardabweichung der Experimente liegt bei 12\%. Die Untergruppe BOY054 liegt parallel zur Symmetrielinie bei $y=54 \mathrm{~mm}$ und ist in Abb. $\mathbf{5 . 1 9}$ aufgetragen.
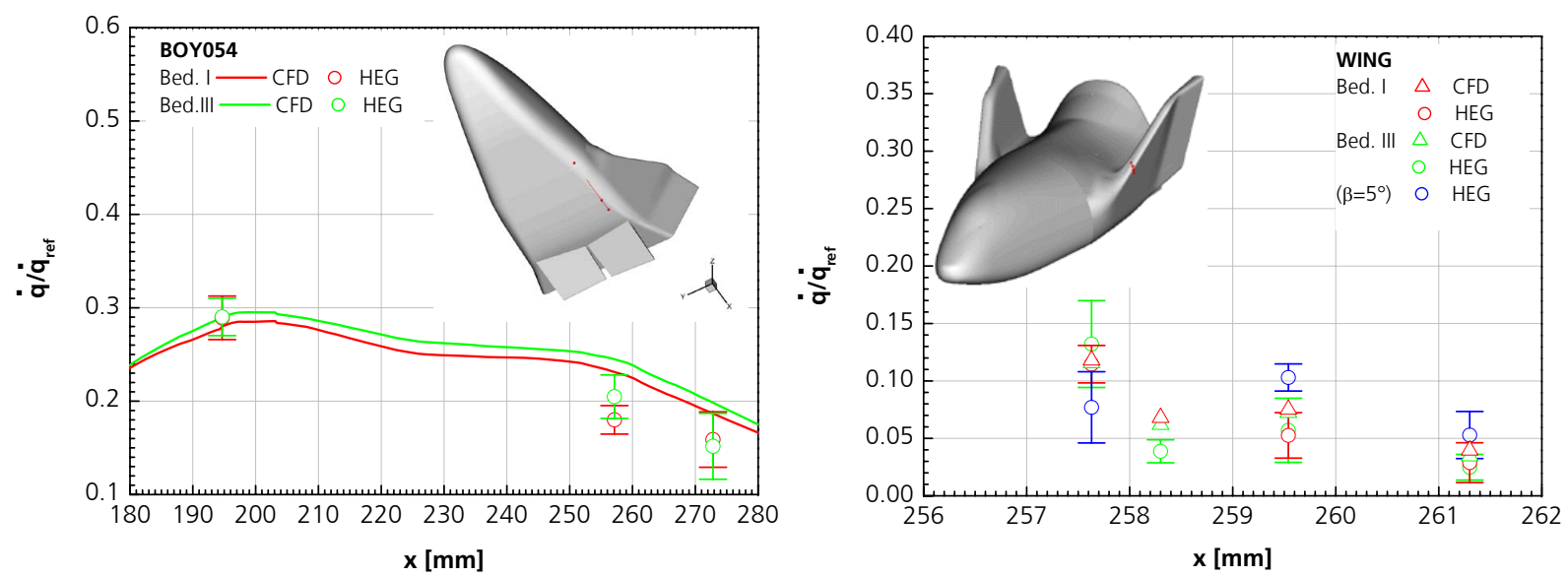

Abbildung 5.19: Wärmestromdichtemessung X-38-Modell (Bed. I,III, Spannw. und Flügel). 
Die größte Abweichung beträgt 16\%, und die Standardabweichung der Experimente liegt bei $12 \%$, wie bei den Schnitten BOX194/BOX257.

Die Ergebnisse für die normierten Wärmestromdichten der Untergruppe WING auf der Vorderkante des Flügels sind in Abb. 5.19 für beide Versuchsbedingungen (I und III) dargestellt. Da diese Sensoren nicht auf einem Schnitt liegen, wurden für den Vergleich die Punktwerte der numerischen Lösungen mit den Messungen zusammen dargestellt. Der generelle Trend der Messungen wird von der Numerik korrekt wiedergegeben. Die mittlere Abweichung der Messungen von den numerischen Werten liegt bei 30\%. Alle vier Sensoren sind in Bereichen großer Wärmestromdichtegradienten auf der Modelloberfläche installiert, so dass selbst kleine Änderungen in der Anströmung zu großen Änderungen in der Wiederholbarkeit der Messergebnisse führen. Die Standardabweichung der Messergebnisse liegt bei 37\%. Die Standardabweichungen der Messungen des Sensors E01 ( $x=257.63 \mathrm{~mm})$ mit und ohne Schiebewinkel überschneiden sich, so dass die Messungen hier keinen Unterschied zeigen. Durch einen Ausfall des Sensors E02 existieren keine Messungen für die Versuche mit Schiebewinkel. Der Sensor E03 $(x=259.54 \mathrm{~mm})$ zeigt für die Messungen mit Schiebewinkel eine höhere Wärmestromdichte, während für die Messungen mit Sensor E04 ( $\mathrm{x}=261.3 \mathrm{~mm}$ ) keine Unterscheidung möglich ist.

Zusammenfassend lässt sich sagen, dass die Druckmessungen auf dem Vorkörper und den Klappen insgesamt eine Wiederholbarkeit von 3\% zeigen. Abweichungen zu den numerische Daten liegen im Bereich von 10\%. Für die Wärmestromdichtemessungen nur des Vorkörpers ist die Wiederholbarkeit mit 10\% schlechter und die Abweichungen zu den numerischen Lösungen mit Annahme laminarer Strömung liegen im Bereich von 15\%.

Die Ergebnisse für die Messungen auf den Klappen zeigen für einen Klappenausschlag von $\delta=20^{\circ}$ ein deutliches Maximum bei oder nach dem Wiederanlegen. Die im Experiment gemessene Überhöhung zu den laminaren Rechnungen beträgt ca. $40 \%$ für beide HEG-Bedingungen. Bei den Messungen mit einem Schiebewinkel von $\beta=5^{\circ}$ und $\delta=20^{\circ}$ Klappenausschlag erhöht sich dieses Maximum noch einmal um 11\%.

Bei $\delta=30^{\circ}$ Klappenausschlag zeigt dieses Maximum eine Überhöhung um den Faktor 2.5 gegeben über der laminaren Rechnung bei beiden HEG Bedingungen. Der Vergleich zu einer transitionellen Rechnungen zeigt Übereinstimmung für das Maximum, allerdings ist der im Experiment gemessene weitere Verlauf der Wärmestromdichte auf der Klappe unterschiedlich zu der Rechnung. Die Rechnung zeigt einen fast konstanten Verlauf, das Experiment einen Abfall und ein Wiederansteigen der Wärmestromdichte. 


\subsection{Doppelrampenmodell}

\subsubsection{Durchgeführte Versuche}

In diesem Abschnitt werden die Messergebnisse diskutiert, die mit dem Doppelrampenmodell gewonnen wurden. Die Ergebnisse für die gemessenen Ablöselängen werden dargestellt und mit bestehenden Korrelationen verglichen. Die gemessenen Wiederanlegewärmestromdichten werden zu den Messungen am X-38-Modell ins Verhältnis gesetzt und diskutiert. In Abb. $\mathbf{5 . 2 0}$ ist die sich ausbildende abgelöste Strömung auf dem Doppelrampenmodell schematisch dargestellt. In der Abbildung 5.20 finden sich auch die in diesem Abschnitt verwendeten Bezeichnungen.

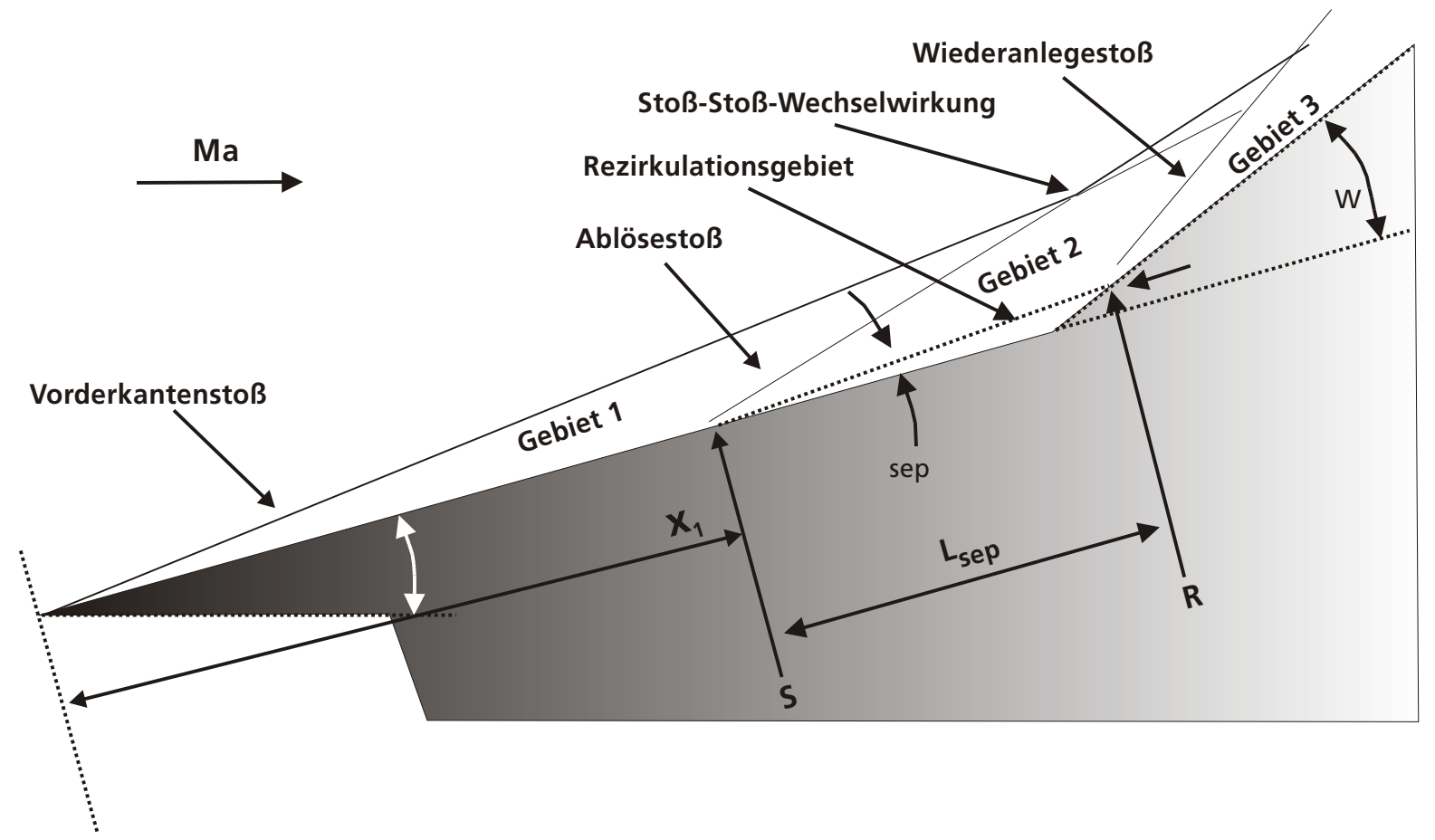

Abbildung 5.20: Nomenklatur der abgelösten Strömung auf dem Doppelrampenmodell.

Die durchgeführten Versuche sind in Tab. 5.7 angegeben. Hier sind die Versuchsnummern, die HEG-Bedingung, der Anstellwinkel der ersten Rampe $\Theta_{1}$, der Rampenwinkel der zweiten Rampe $\Theta_{2}$ und der sich hieraus ergebene Umlenkwinkel $\Theta_{W}$ der zweiten Rampe angegeben. Die Distanz $x_{1}$ ist die Vorlauflänge der Strömung auf der ersten Rampe bis zum Punkt der Ablösung, die Länge der ersten Rampe ist mit $L_{1}$ bezeichnet. Die weiteren Größen werden weiter unten erläutert. 


\begin{tabular}{|c|c|c|c|c|c|c|c|c|c|c|c|c|c|c|c|}
\hline Versuch & Bedingung & $\Theta_{1}$ & $\Theta_{2}$ & $\Theta_{\mathrm{w}}$ & $\mathrm{x}_{1} / \mathrm{L}_{1}$ & $1+\left(L_{R} / L_{1}\right)$ & Lsep $/ \mathrm{L}_{1}$ & $\Delta\left(\right.$ Lsep/ $\left./ L_{1}\right)$ & $\Theta_{\text {sep }}$ & $\mathbf{p}_{1} / \mathbf{p}_{\mathrm{t} 2}$ & $q_{1} / q t_{2}$ & $\mathbf{p}_{\mathrm{pL}} / \mathbf{p}_{\mathrm{t} 2}$ & $\mathrm{p}_{\mathrm{R}} / \mathrm{pt}_{2}$ & $\mathrm{p}_{\mathrm{pk}} / \mathrm{p}_{\mathrm{t} 2}$ & $\mathrm{q}_{\mathrm{pk}} / \mathrm{q}_{\mathrm{t} 2}$ \\
\hline 446 & III & 0 & 0 & 0 & & & & & & & & & & & \\
\hline 455 & III & 15 & 25 & 10 & & & & & & 0.111 & 0.0633 & & & 0.277 & 0.104 \\
\hline 454 & III & 15 & 30 & 15 & & & & & & 0.111 & 0.0633 & & & 0.490 & 0.165 \\
\hline 453 & III & 15 & 35 & 20 & 0.645 & 1.085 & 0.435 & 0.0187 & 3.82 & 0.111 & 0.0633 & 0.185 & 0.270 & 0.626 & 0.345 \\
\hline 452 & III & 15 & 40 & 25 & 0.575 & 1.115 & 0.531 & 0.0190 & 5.25 & 0.111 & 0.0633 & 0.160 & 0.232 & 0.877 & 0.635 \\
\hline 447 & III & 15 & 50 & 35 & 0.435 & 1.173 & 0.714 & 0.0204 & 4.58 & 0.111 & 0.0633 & 0.191 & 0.265 & 1.477 & 1.403 \\
\hline 459 & III & 20 & 30 & 10 & 0.859 & 1.045 & 0.185 & 0.0187 & 2.41 & 0.172 & 0.0864 & 0.261 & 0.288 & 0.610 & 0.222 \\
\hline 458 & III & 20 & 35 & 15 & 0.741 & 1.038 & 0.296 & 0.0184 & 1.90 & 0.172 & 0.0864 & 0.262 & 0.293 & 0.834 & 0.419 \\
\hline 460 & III & 20 & 40 & 20 & 0.671 & 1.012 & 0.340 & 0.0176 & 0.69 & 0.172 & 0.0864 & 0.274 & 0.287 & 1.137 & 0.627 \\
\hline 456 & I & 15 & 25 & 10 & & & & & & 0.108 & 0.0518 & 0.135 & & 0.284 & 0.106 \\
\hline 457 & I & 15 & 35 & 20 & 0.859 & 1.035 & 0.227 & 0.0182 & 3.94 & 0.108 & 0.0518 & 0.179 & 0.219 & 0.617 & 0.218 \\
\hline 494 & I & 15 & 40 & 25 & 0.575 & 1.150 & 0.570 & 0.0195 & 6.64 & 0.108 & 0.0518 & 0.230 & 0.209 & 1.772 & 0.428 \\
\hline 491 & I & 20 & 45 & 25 & 0.763 & 1.141 & 0.370 & 0.0199 & 9.28 & 0.169 & 0.0730 & 0.321 & 0.728 & 1.186 & 0.551 \\
\hline 492 & 1 & 20 & 50 & 30 & 0.584 & 1.192 & 0.590 & 0.0215 & 9.36 & 0.169 & 0.0730 & 0.332 & 1.036 & 2.049 & 0.935 \\
\hline 493 & 1 & 20 & 55 & 35 & 0.523 & 1.243 & 0.690 & 0.0235 & 11.65 & 0.169 & 0.0730 & 0.345 & 1.124 & 2.863 & 0.858 \\
\hline
\end{tabular}

Tabelle 5.7: Übersicht über die durchgeführten Doppelrampenversuche.

Auf der Oberfläche des Doppelrampenmodells wurden Druck und Wärmestromdichte in der Symmetrielinie gemessen und Schlierenfilme mit Hilfe der HochgeschwindigkeitsSichtbarmachung aufgenommen. Die Schlierenfilme wurden benutzt, um die zeitliche Entwicklung der Ablöseblase zu beurteilen. Unter Verwendung des jeweiligen Schlierenbildes zur Messzeit wurden der Ablöseort auf der ersten Rampe und der Wiederanlegepunkt der Strömung auf der zweiten Rampe bestimmt. Um die Grenzschicht auf dem Doppelrampenmodell charakterisieren zu können, wurde die Umströmung der ersten Rampe für die Anstellwinkel $\Theta_{1}$ von $10^{\circ}$ und $20^{\circ}$ für beide verwendete HEG-Bedingungen (I und III) numerisch bestimmt. Die Anströmung für diese Rechnungen wurde mit Hilfe von Düsenrechnungen bestimmt. Für diese Rechnungen wurden gemittelte Reservoirbedingungen (s. auch Abschnitt 3.3.4) benutzt, so dass die Keil-Rechnungen ein Mittel aller durchgeführten Versuche repräsentieren. Aus den Rechnungen wurden die benötigten Werte für die in Abschnitt 2.4 vorgestellten Korrelationen bestimmt.

\subsubsection{Zeitlicher Aufbau der Strömung}

Um die zeitliche Entwicklung der Ablöseblase zu beurteilen und hiermit sicher zustellen, dass sich ein stationäres Strömungsfeld während der kurzen Messzeit ausbildet, wurde die Entwicklung der Strömung mit Hilfe der Hochgeschwindigkeits-Sichtbarmachung aufgezeichnet. In der Abb. $\mathbf{5 . 2 1}$ ist die zeitliche Entwicklung exemplarisch für die Versuchsbedingung III auf den Einzelbildern dargestellt. Die angegebenen Zeiten beziehen sich auf den Zeitpunkt der Stoßreflektion am Stoßrohrende (SR). Zum Vergleich findet sich in Abb. $\mathbf{5 . 2 2}$ die Strömungsentwicklung für die HEG Versuchsbedingung I. Um einen Eindruck über die zeitliche Entwicklung der Druck- und Wärmestromdichteverteilungen auf dem Modell zu geben, wurden die Messwerte alle 0.2 ms über 0.2 ms gemittelt. Die Darstellung dieser Auswertung findet sich in Abb. $\mathbf{5 . 2 3}$ für die Versuchsbedingung III und in $\mathbf{A b b .} \mathbf{5 . 2 4}$ für die Versuchsbedingung I. 

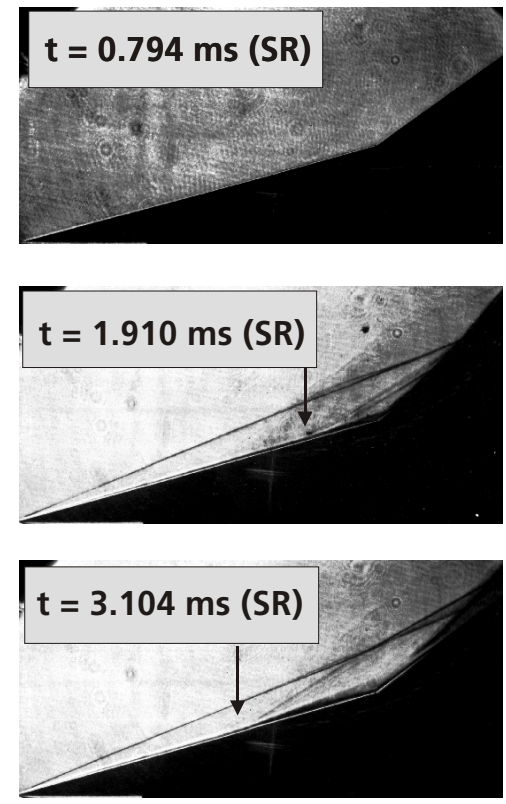
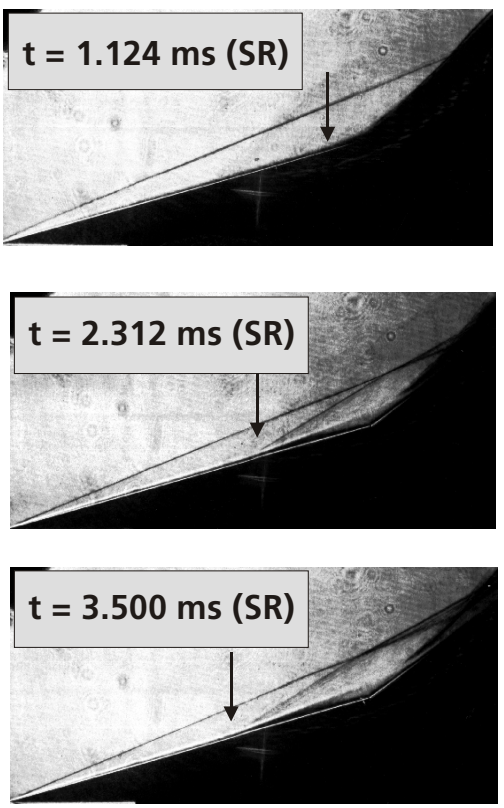
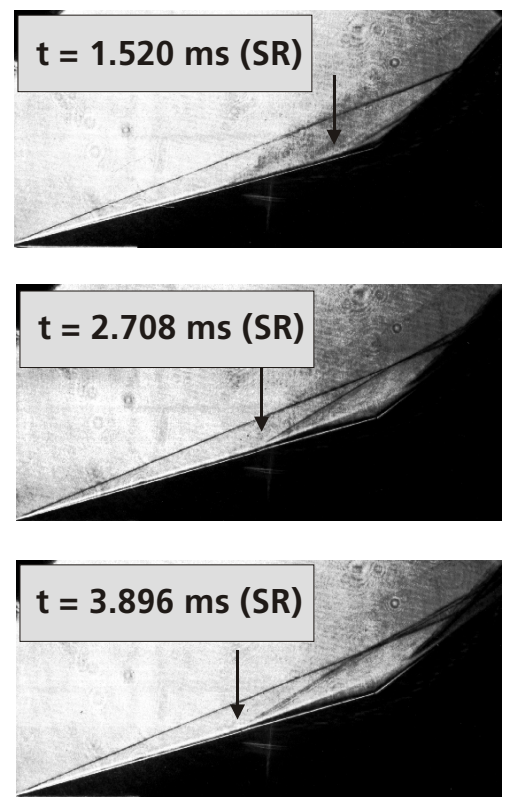

Abbildung 5.21: Hochgeschwindigkeits-Sichtbarmachung des Aufbaus der Strömung auf dem Doppelrampenmodell bei HEG Versuchsbedingung III (Versuch 453).
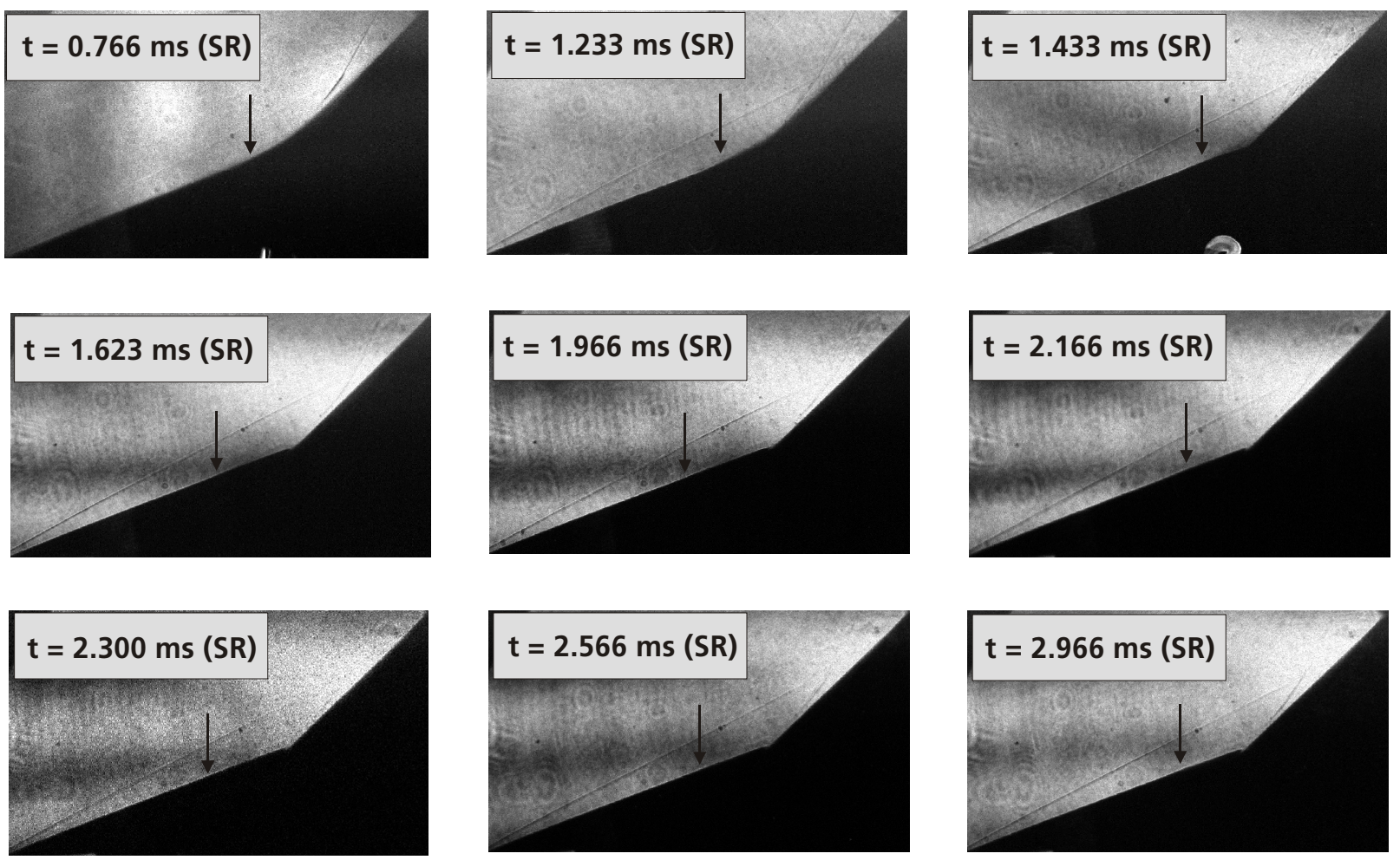

Abbildung 5.22: Hochgeschwindigkeits-Sichtbarmachung des Aufbaus der Strömung auf dem Doppelrampenmodell bei der HEG-Versuchsbedingung I (Versuch 491). 

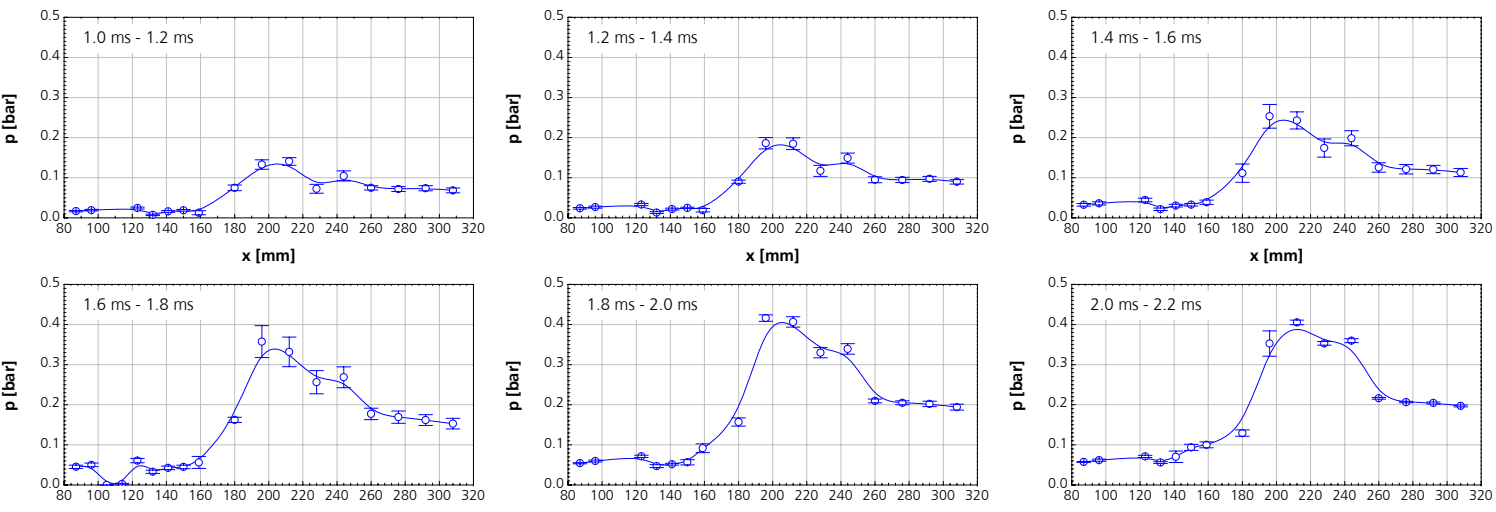

$\mathbf{x}[\mathrm{mm}]$

$\mathrm{x}[\mathrm{mm}]$
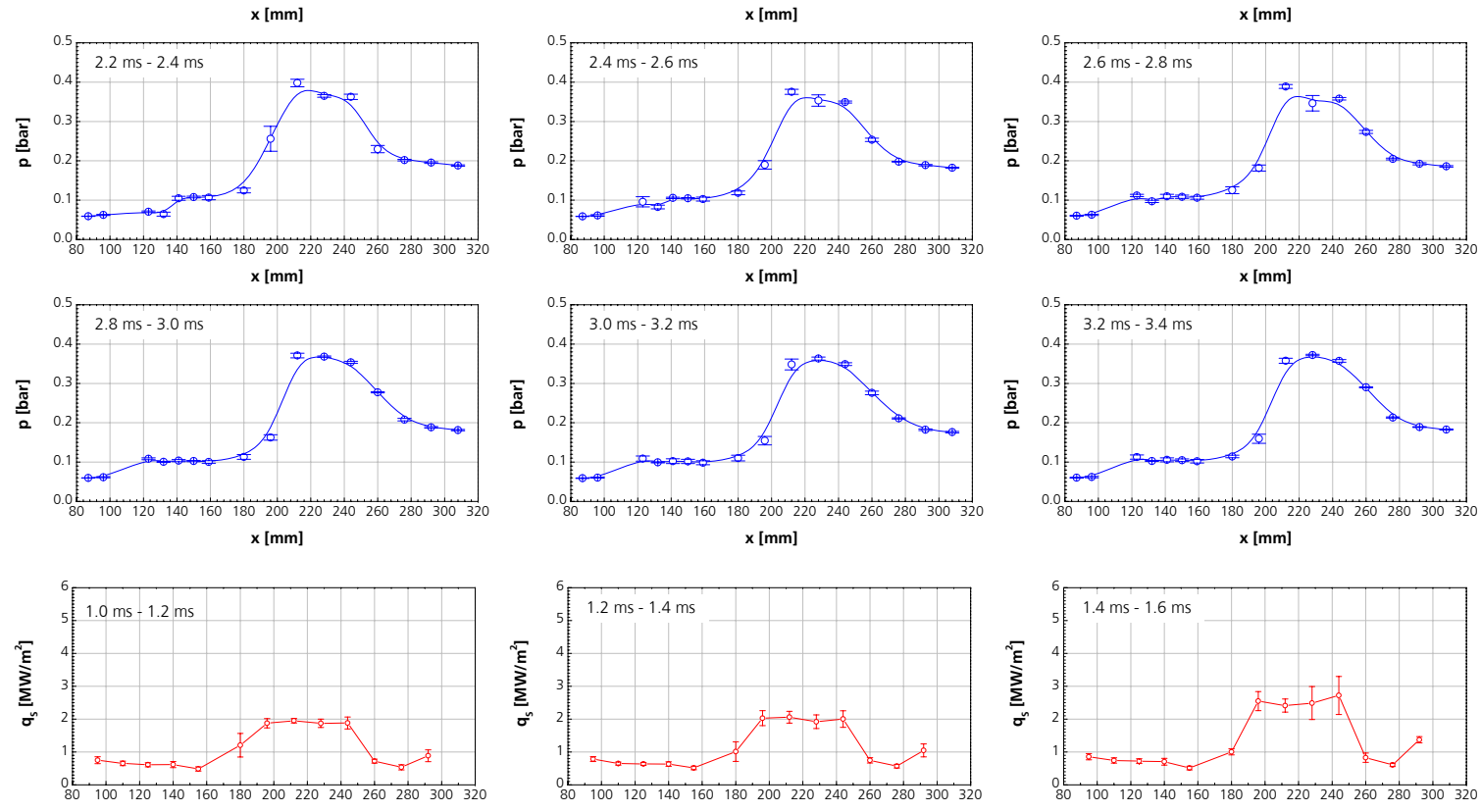

$\mathrm{x}[\mathrm{mm}]$

$\mathrm{x}[\mathrm{mm}]$
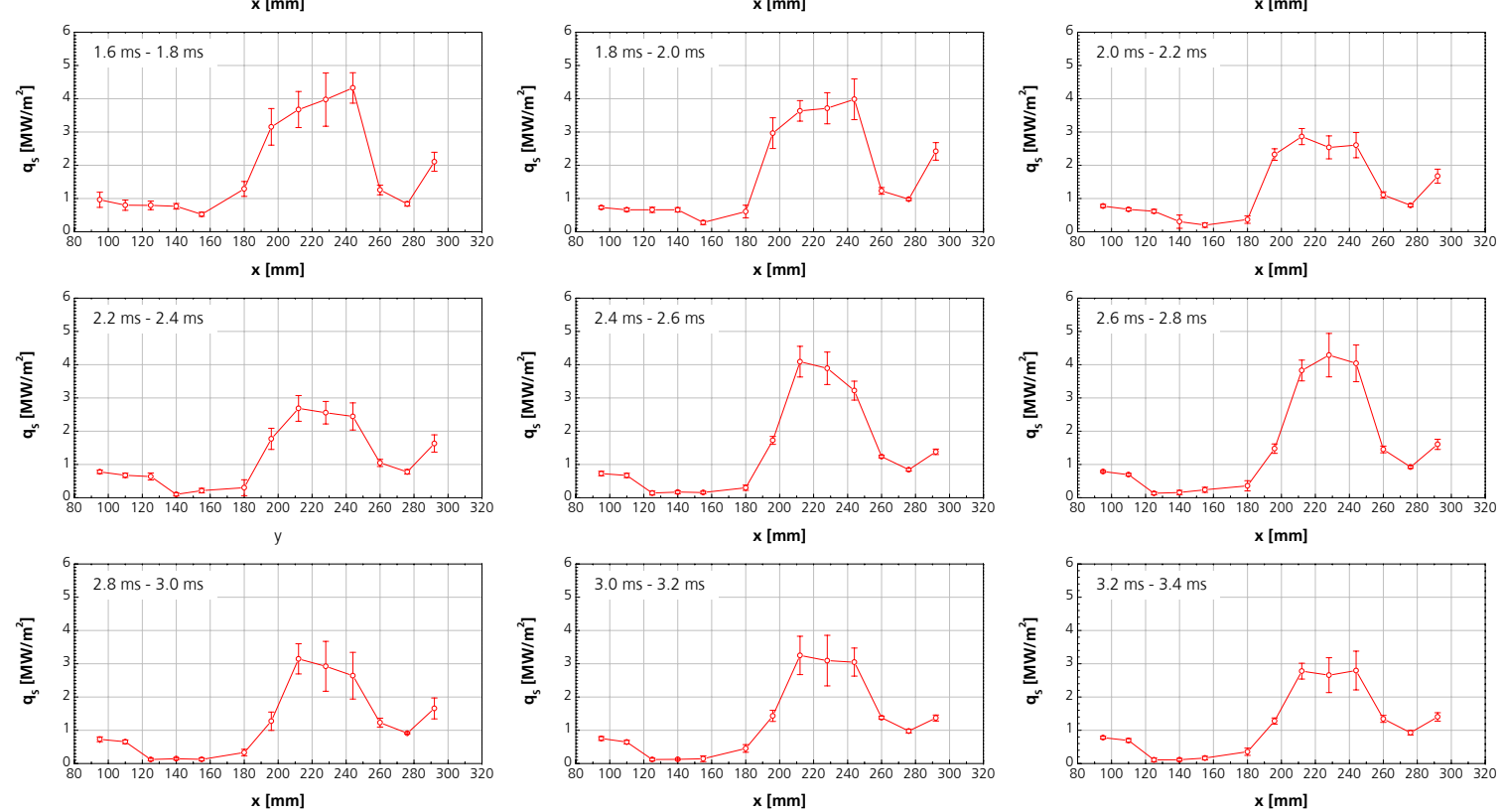

Abbildung 5.23: Zeitliche Entwicklung der Druck und Wärmestromdichteverteilungen auf dem Doppelrampenmodell bei der HEG-Versuchsbedingung III (Versuch 453). 

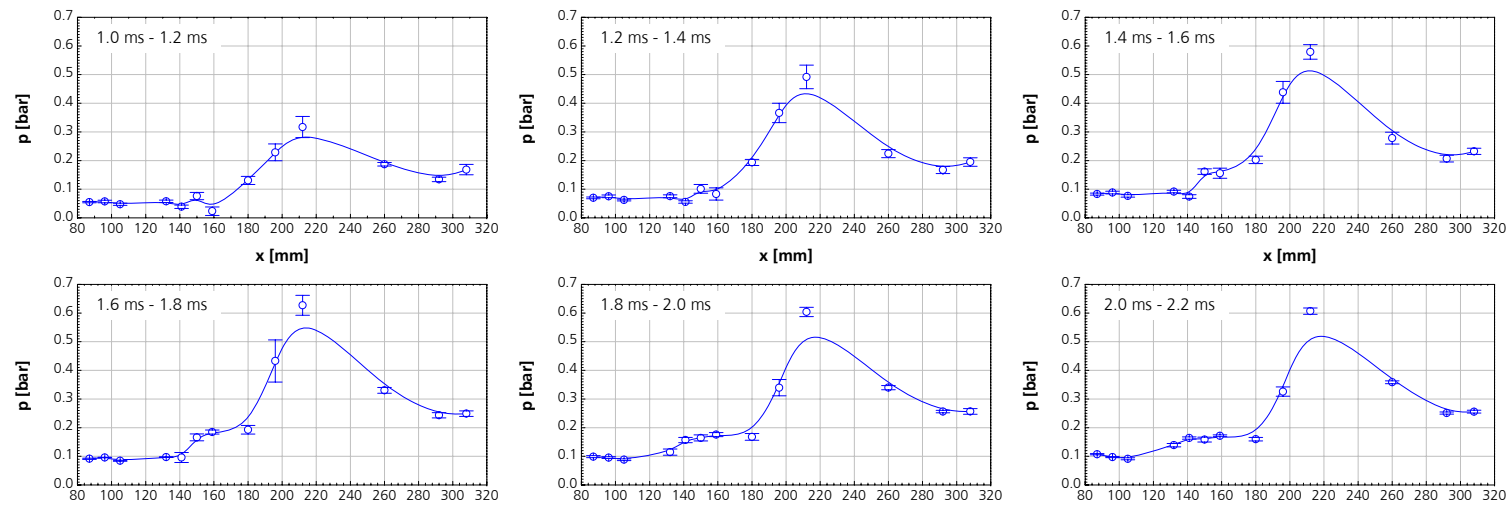

$\mathbf{x}[\mathrm{mm}]$

$x[\mathrm{~mm}]$

$x$ [mm]
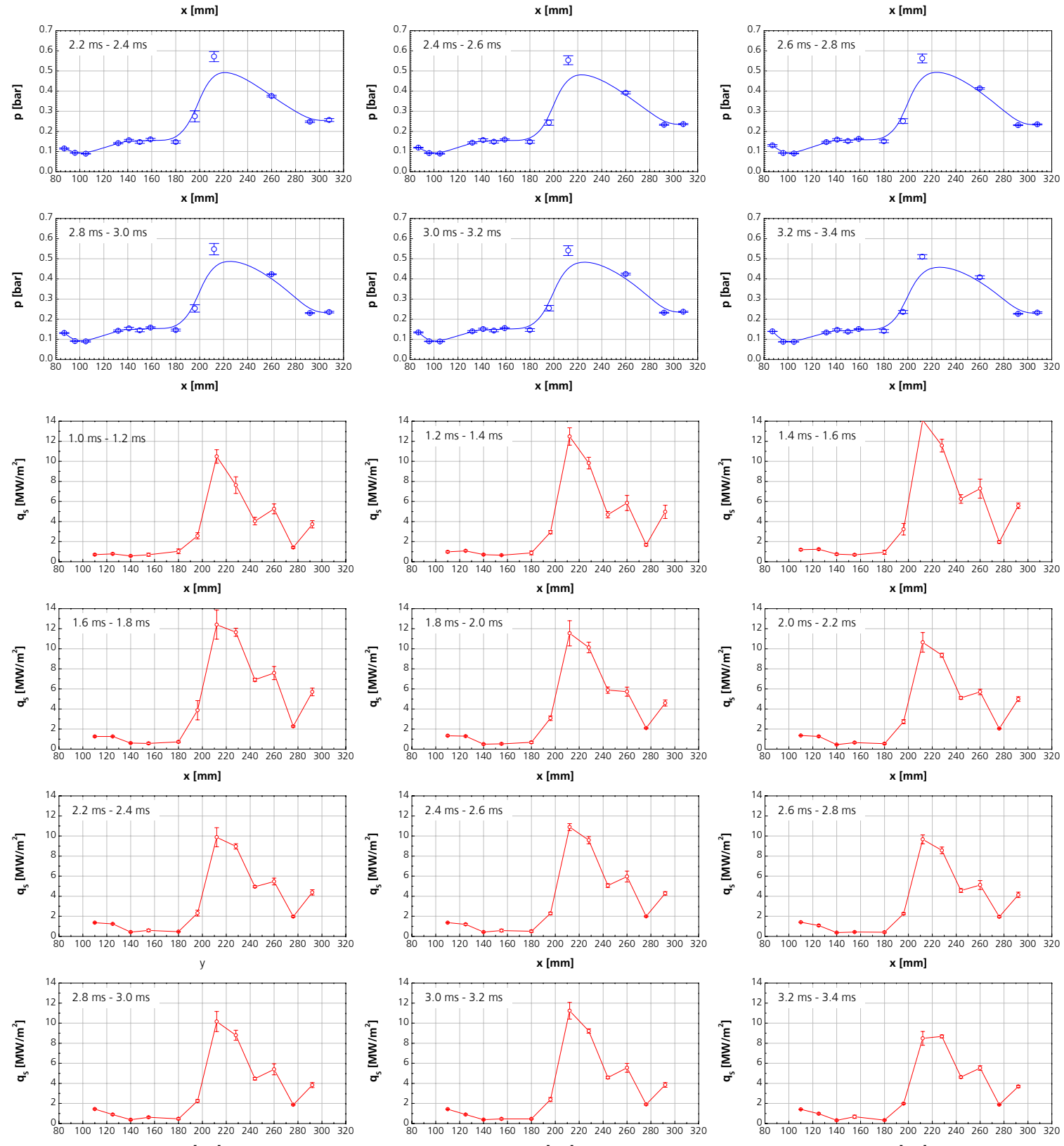

Abbildung 5.24: Zeitliche Entwicklung der Druck- und Wärmestromdichteverteilungen auf dem Doppelrampenmodell bei der HEG-Versuchsbedingung I (Versuch 491). 
Durch Vermessung jedes einzelnen Bildes der Schlierenfilme, die aufgenommen wurden, kann die Ablöselänge $L_{s e p}$ in der zeitlichen Entwicklung aufgetragen und beurteilt werden. Die Auswertung für die beiden exemplarischen Versuche 453 und 491 ist in der Abb. $\mathbf{5 . 2 5}$ gegeben. Die Auswertung wurde mittels des in Abschnitt 5.3.3 beschriebenen Verfahrens durchgeführt. Hier ist die zeitliche Entwicklung der Ablöselänge $L_{\text {sep }}$ für die Bedingung III auf der linken Seite und für die Versuchsbedingung I auf der rechten Seite aufgetragen. Es ist zu erkennen, dass sich die Ablöseblase bei der Versuchsbedingung I schneller aufbaut. Während die Ablöseblase eine konstante Länge ab einem Zeitpunkt von ca. 3 ms nach Stoßreflektionszeitpunkt aufweist, ist diese Konstanz bei der Versuchsbedingung I schon nach ca. 2 ms nach Stoßreflektionszeitpunkt gegeben. Dieses Verhalten wird bei allen durchgeführten Versuchen beobachtet. Ein Grund hierfür könnte sein, dass die absolute Geschwindigkeit der freien Anströmung bei der Versuchsbedingung I größer ist als bei der Versuchsbedingung III. Weiterhin vergrößert sich die Ablöseblase schlagartig ab 2.6 ms nach Stoßreflektionszeitpunkt für die Versuchsbedingung I. Auch dieses Verhalten wird bei allen Experimenten mit dieser Versuchsbedingung beobachtet. Hier wird vermutet, dass bereits eine Verunreinigung mit dem Treibgas der Anlage vorliegt, eine Versuchsauswertung zu diesem Zeitpunkt kann somit nicht mehr stattfinden. Durch die Vermischung der Luft mit dem Treibgas Helium und Argon vergrößert sich das effektive Verhältnis der spezifischen Wärmen $\kappa_{1}$ des Gasgemisches, was wiederum ein Absinken der lokalen Machzahl $M_{1}$ zur Folge hat. Dieses Absinken der lokalen Machzahl $M_{1}$ bedingt dann die Vergrößerung der Ablöseblase. Wie schon in Abschnitt 2.4 dargestellt, wurde dieses Verhalten bei allen Arten von abgelösten Strömungen beobachtet, unabhängig davon, ob Turbulenz vorlag oder nicht.
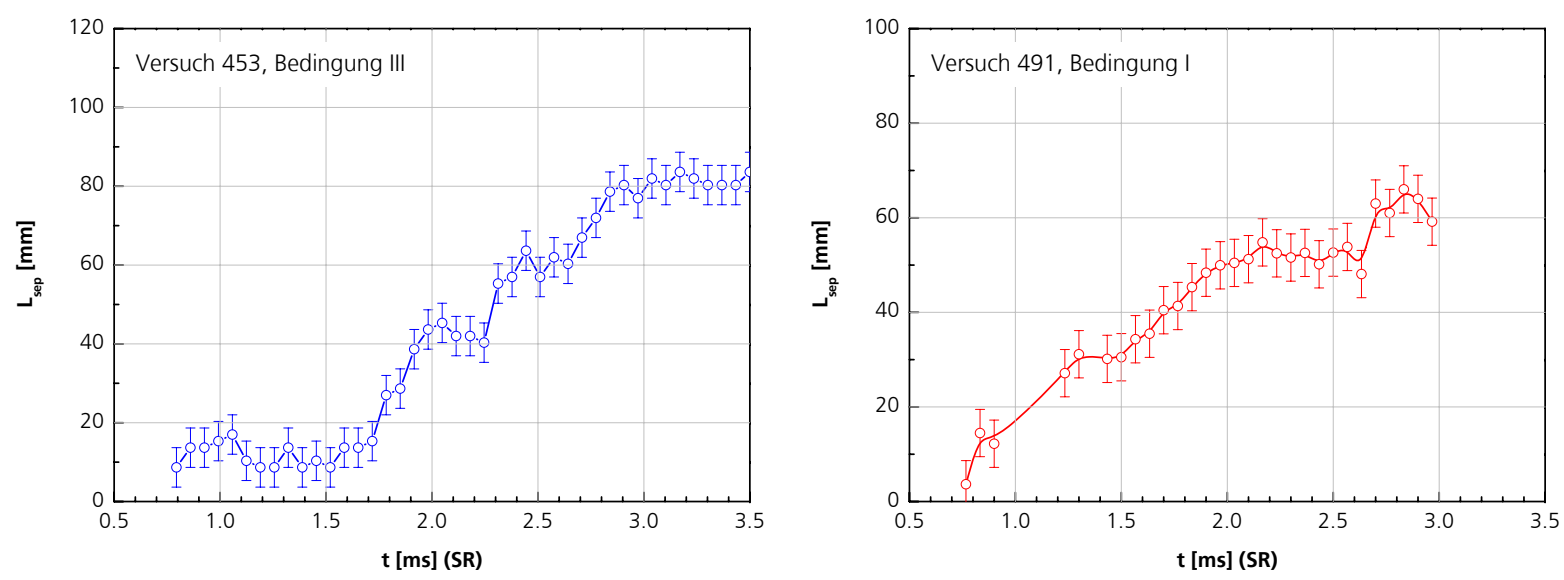

Abbildung 5.25: Zeitliche Entwicklung der Ablöselänge $L_{\text {sep }}$ für die HEG-Versuchsbedingung III bei Versuch 453 (links) und die HEG-Versuchsbedingung I bei Versuch 491 (rechts).

\subsubsection{Bestimmung der Ablöselänge}

Das Verfahren zur Bestimmung der Ablöselänge ist für den Versuch 453 (exemplarisch für die restlichen Versuche) in Abb. $\mathbf{5 . 2 6}$ dargestellt. Die weiße gestrichelte Linie im Schlierenbild gibt die Lage der Scherschicht an; die roten Linien die Lage der Rampenoberfläche. Die 
Schnittpunkte zwischen der weißen gestrichelten Linie und der Modelloberfläche geben die Lage der Ablösung S und die Lage des Wiederanlegens R der Strömung an.

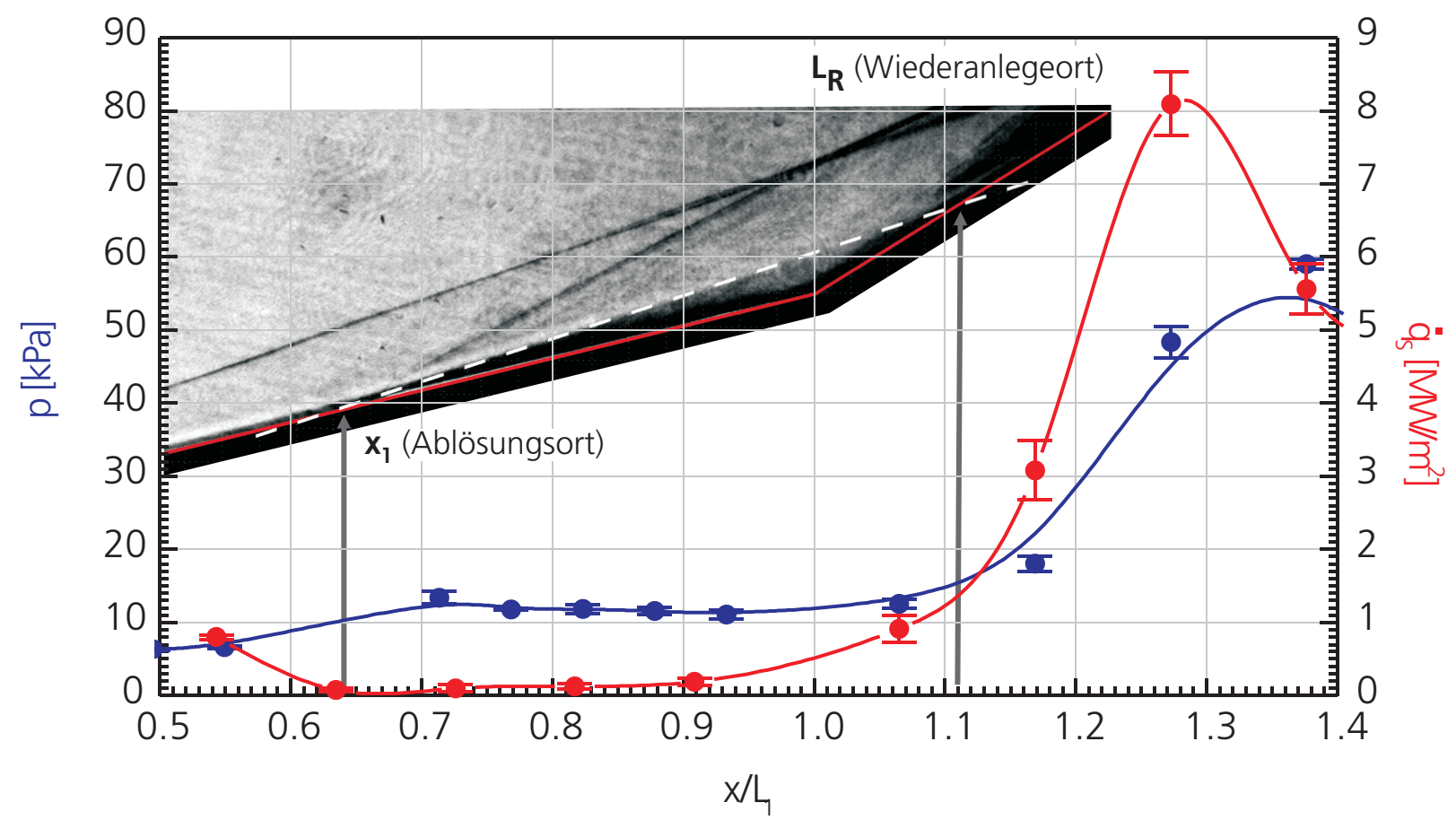

Abbildung 5.26: Veranschaulichung der Messmethode für die Ablöselänge bei Versuch 453. Die durchgezogenen Linien sind Spline-Interpolationen der Messwerte und dienen nur zur optischen Trennung beider Verläufe.

Die so ermittelten Positionen ( $x_{1}$ für den Ablöseort und $L_{R}$ für den Wiederanlegeort) sind in Tab. 5.7 eingetragen. Die Ablöselänge $L_{\text {sep }}$ und der Winkel $\Theta_{\text {sep }}$, unter dem die Scherschicht zu der Oberfläche der ersten Rampe liegt, ergeben sich dann rechnerisch. Alle Längenwerte in Tab. 5.7 sind mit der Länge der ersten Rampe $L_{1}$ entdimensionalisiert. Die Messergebnisse für die Wärmestromdichte und den Druck auf der Oberfläche der Rampe sind ebenfalls in der Abb. 5.26 aufgetragen. Die Messpositionen wurden mit den Anstellwinkeln der ersten und der zweiten Rampe umgerechnet, um die eindimensionale Messaufnehmerverteilung mit dem zweidimensionalen Schlierenbild vergleichen zu können. Die durchgezogenen Linien durch die Messwerte sind Spline-Interpolationen und dienen nur dazu, den Verlauf der Messwerte besser sichtbar zu machen. Der Druckverlauf in Abb. 5.26 zeigt ein Ansteigen des Druckes hinter dem Ablösepunkt und verbleibt dann in der abgelösten Region auf einem konstanten Druck, dem sogenannten Plateaudruck. Auch nach der Scharnierlinie bei $x=1$ steigt der Druck nicht an. Er verbleibt auf dem Plateauwert, um dann nach dem Wiederanlegen zu steigen. Die Wärmestromdichtemessungen zeigen einen typischen Abfall beim Ablösen der Strömung, um dann kurz vor dem Wiederanlegepunkt zu steigen. Das Sinken der Wärmestromdichte hinter der Ablösung ist konsistent mit Beobachtungen, die bei rein laminaren Ablösungen gemacht wurden (Needham (1965)). Die Erhöhung der Wärmestromdichte kurz vor dem Wiederanlegen wurde schon von Kumar u. Stollery (1994) beobachtet und zwar dann, wenn ein Übergang von laminarer zu turbulenter Strömung in 
der abgelösten Scherschicht stattfindet. Die Diskussion über diese Beobachtung wird weiter unten fortgeführt. Die Ablöselänge wird wie in Abb. $\mathbf{5 . 2 7}$ skizziert über

$$
L_{\text {sep }}=\sqrt{\left(L_{u} \cos \Theta_{1}+L_{R} \cos \Theta_{2}\right)^{2}+\left(L_{u} \sin \Theta_{1}+L_{R} \sin \Theta_{2}\right)^{2}}
$$

bestimmt. Hier ist $L_{u}$ der Abstand zwischen Ablösung und der Scharnierlinie, und es gilt $L_{u}=1-L_{R}$. Die Fehlerfortpflanzung, mit der die Fehler für $L_{s} e p$ bestimmt werden, findet sich im Anhang in Abschnitt B.2. Die ermittelten Fehler $\Delta L_{\text {sep }}$ sind in Tab. 5.7 angegeben und in den Auftragungen mit eingetragen.
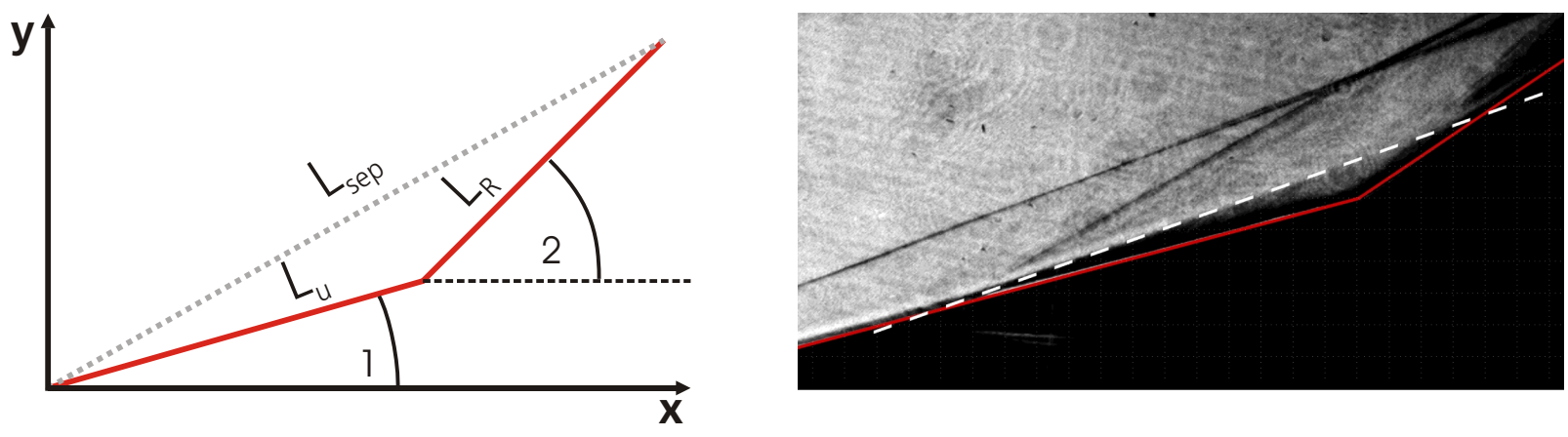

Abbildung 5.27: Koordinatensystem für die Bestimmung der Ablöselänge.

\subsubsection{Messung der Oberflächenwerte}

Alle gemessenen Druckverläufe sind in Abb. $\mathbf{5 . 2 8}$ dargestellt. Auch hier sind die durchgezogenen Linien wieder Interpolationen der Messwerte und dienen nur dazu, die Verläufe besser unterscheiden zu können. Die Werte für die Positionen der Sensoren auf den beiden Rampen sind mit der Länge der ersten Rampe $L_{1}$ entdimensionalisiert; der Wert $x / L_{1}=1$ gibt die Lage der Scharnierlinie an. Die Messwerte für die Oberflächendrücke sind mit dem gemessenen Pitot-Druck des jeweiligen Versuches normiert. Die numerischen Oberflächendrücke aus den Keilrechnungen für die ersten Rampe sind zusätzlich in der Abb. 5.28 dargestellt. Aus der in der Numerik verwendeten Anströmung wurde mit der Pitot-RayleighFormel (NACA1135 (1953), Gl. 100) der Referenzwert für die Normierung bestimmt. Auch die Orte der ermittelten Ablösepunkte $x_{1}$ und die Wiederanlegeorte $L_{R}$ sind in der Abb. 5.28 als Dreiecke eingetragen.

Alle Messungen zeigen, wenn die Strömung ablöst, den typischen Plateaudruck $p_{P L}$, der in Tab. 5.7 zu finden ist. Der Oberflächendruck beim Wiederanlegen $p_{R}$ ist in Tabelle 5.7 eingetragen. Dieser Wert wurde als Mittel der beiden Sensoren, zwischen denen der Wiederanlegepunkt liegt, bestimmt. Nach dem Wiederanlegen zeigt der Druckverlauf ein Maximum. Der Maximalwert $p_{p k}$ (Tab. 5.7) ist der Messwert auf der Oberfläche, der den höchsten Druck anzeigt. Bei HEG-Bedingung I mit $\Theta_{1}=15^{\circ}$ konnte für eine Umlenkung von $\Theta_{W}=10^{\circ}$ keine Ablöselänge bestimmt werden, da auf den Schlierenbildern der Strömung keine Ablösung erkennbar ist. Das Druckverhältnis zwischen erster und zweiter Rampe mit einem 



Abbildung 5.28: Normierte Oberflächenmessungen für den Druck für alle Versuche. Zusätzlich eingetragen die Lösung der Rechnungen für einen Keil mit einem dem Experiment entsprechenden Anstellwinkel der ersten Rampe $\Theta_{1}$. Die durchgezogenen Linien sind Spline-Interpolationen und dienen der Unterscheidbarkeit der Verläufe.

Wert von 3.130 entspricht ungefähr einer Umlenkung der Strömung um 10. Da auch keine Drucküberhöhung beim Wiederanlegen auftritt, kann angenommen werden, dass bei diesem Experiment keine Ablösung auftrat. Ein ähnliches Verhalten zeigt die Messung bei HEG-Bedingung III, $\Theta_{1}=15^{\circ}$ mit einer Umlenkung $\Theta_{W}=10^{\circ}$ in Abb. 5.28. Im Gegensatz hierzu liegt bei HEG-Bedingung III, $\Theta=20^{\circ}$ für eine identische Umlenkung eindeutig eine Ablösung vor. Katzer (1989) fand bei seinen Experimenten, dass sich der Druckunterschied ${ }^{1}$, bei dem Ablösung eintritt, mit der Machzahl der Außenströmung $M_{1}$ vor der Ablösung vergrößert. Die beiden Fälle für eine Umlenkung von $\Theta_{W}=10^{\circ}$ entsprechen den Werten von $M_{1}=5.24 / \Theta_{1}=15^{\circ}$ und $M_{1}=4.37 / \Theta_{1}=20^{\circ}$ (s. Tab. 5.8). Dass sich der Umlenkwinkel, bei dem Ablösung stattfindet, bei HEG-Bedingung III und $\Theta_{1}=20^{\circ}$ verringert, ist somit konsistent zu den Ergebnissen von Katzer.

Bei den Versuchen mit HEG-Bedingung I (höchste Ruheenthalpie) und bei einem Rampenwinkel von $\Theta_{1}=20^{\circ}$ zeigt die Ablösung beim Übergang der Umlenkung von $\Theta_{W}=30^{\circ}$ auf $\Theta_{W}=35^{\circ}$ ein abweichendes Verhalten im Vergleich zu den anderen Experimenten. Die Län-

\footnotetext{
${ }^{1}$ Entspricht dem Umlenkwinkel $\Theta_{W}$.
} 
ge der Ablösung ändert sich kaum, und weiterhin ist ein starker Druckabfall nach dem Wiederanlegen zu sehen. Dieses Verhalten ist bei keiner der anderen Messreihen festzustellen.

Die Ergebnisse für die Wärmestromdichtemessungen sind in $\mathbf{A b b .} \mathbf{5 . 2 9}$ dargestellt, analog zu denen der Druckmessungen. Ebenfalss enthalten ist findet sich die entdimensionalisierte Wärmestromdichte aus den Keilrechnungen. Die Werte in Abb. 5.29 sind mit den Wärmestromdichtemessungen auf der Halbkugel der permanenten Sonde entdimensionalisiert worden. Für den Referenzwert der numerischen Daten wurde die Korrelation von Verant (1995) (s. a. Abschnitt 3.3.5) zur Bestimmung der Wärmestromdichte herangezogen. Wie bei den Druckergebnissen sind in der Abb. 5.29 die Lagen der Ablösung und des Wiederanlegens der Strömung mit den Dreiecken bezeichnet. Aus den Wärmestromdichtemessungen wurde der Maximalwert $\dot{q}_{p k}$ nach dem Wiederanlegen entnommen und ist in Tab. 5.7 gelistet. Auch in den Wärmstrommessungen macht sich das abweichende Verhalten im Versuch 492 (HEG-Bedingung III, $\Theta_{1}=20^{\circ}$ ) bemerkbar. Der Wärmestromdichteverlauf nach dem Anlegen der Strömung zeigt deutliche Unterschiede zu den beiden anderen Versuchen dieser Messreihe.
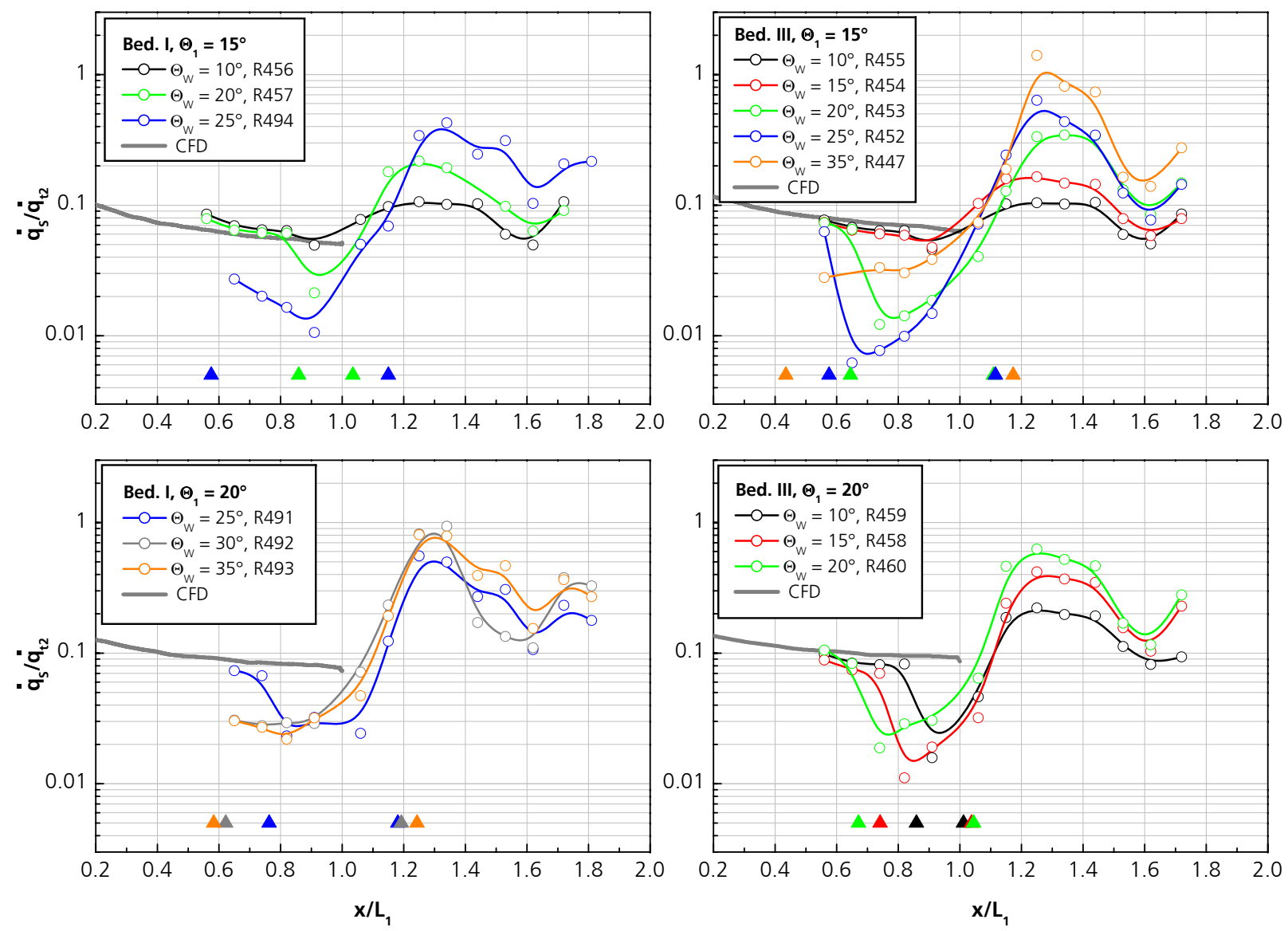

Abbildung 5.29: Normierte Wärmestromdichtemessungen für alle Doppelrampenversuche. Zusätzlich eingetragen die Lösung der Rechnungen für einen Keil mit einem dem Experiment entsprechenden Anstel/winkel der ersten Rampe $\Theta_{1}$. Die durchgezogenen Linien sind Spline-Interpolationen und dienen der Unterscheidbarkeit der Verläufe. 


\subsubsection{Ablöselänge}

Um die Messergebnisse der Umströmung der Doppelrampe mit existierenden Ergebnissen vergleichen zu können, sind Größen und Kennzahlen nötig, die sich nicht experimentell ermitteln lassen. Diese Kennzahlen wurden deshalb aus den Keilrechnungen bestimmt. In Tab. 5.8 sind neben den Verhältnissen der Messwerte aus Tab. 5.7 auch die Werte für die Machzahl $M_{1}$, die Reynoldszahl $R e_{1}$, das effektive Verhältnis der spezifischen Wärmen $\kappa_{1}$ hinter dem Stoß für die Region 1 (s. Abschnitt 2.4) angegeben. Der Chapman-RubesinParameter $C$ setzt Temperatur und Viskosität über den ersten Stoß in die Region 1 ins Verhältnis. Das Verhältnis $\left(p_{3} / p_{2}\right)_{S S}$ ist das Druckverhältnis, das sich durch die Umlenkung der zweiten Rampe aus den Beziehungen für einen schiefen Verdichtungsstoß (NACA1135 (1953), Gl. 128) ergibt. Für die Bestimmung wurde das effektive $\kappa_{1}$ aus den Keilrechnungen benutzt. Für die Umlenkung der Strömung mit $\Theta_{\text {sep }}$ über den Ablösepunkt ist das Verhältnis $\left(p_{3} / p_{2}\right)_{s S}$ nach derselben Verfahrensweise bestimmt worden. Weitere Werte, die den numerischen Daten der Keilrechnungen entnommen wurden, sind die Grenzschichtverdrängungsdicke $\delta_{x 1}^{+}$, die Machzahl $M_{x 1}^{+}$, die Temperatur $T_{x 1}^{+}$und die Reynoldszahl $R e_{x 1}^{+}$am Ort der Ablösung in Strömungsrichtung und am Außenrand der Grenzschichtverdrängungsdicke.

Mit den experimentell bestimmten Längen der Ablösung und den aus den Keilrechnungen ermittelten Kennzahlen können die Experimente mit dem Doppelrampenmodell mit den in der Literatur gefundenen Ergebnissen verglichen werden. Wie in Abschnitt 2.4 erläutert,

\begin{tabular}{|c|c|c|c|c|c|c|c|c|c|c|c|c|c|}
\hline Versuch & Bedingung & $p_{R} / p_{1}$ & $\mathrm{p}_{\mathrm{R}} / \mathrm{p}_{\mathrm{PL}}$ & \multicolumn{2}{|c|}{$\left(p_{R} / p_{P L}\right)^{2}$} & $\mathrm{p}_{\mathrm{pk}} / \mathrm{p}_{1}$ & $\mathrm{q}_{\mathrm{pk}} / \mathrm{q}_{1}$ & $M_{1}$ & $\mathrm{Re}_{1}$ & $\kappa_{1}$ & $\mathrm{C}$ & $\left(p_{3} / p_{2}\right)_{s s}$ & $\left(p_{2} / p_{1}\right)_{s s}$ \\
\hline 446 & III & & & & & & & & & & & & \\
\hline 455 & III & & & & & 2.495 & 1.649 & 5.24 & 167714 & 1.327 & 1.274 & & \\
\hline 454 & III & & & & & 4.418 & 2.606 & 5.24 & 167714 & 1.327 & 1.274 & & \\
\hline 453 & III & 2.438 & 1.461 & \multicolumn{2}{|c|}{2.134} & 5.652 & 5.445 & 5.24 & 167714 & 1.327 & 1.274 & 7.03 & 1.58 \\
\hline 452 & III & 2.093 & 1.449 & \multicolumn{2}{|c|}{2.101} & 7.911 & 10.027 & 5.24 & 167714 & 1.327 & 1.274 & 9.78 & 1.91 \\
\hline 447 & III & 2.393 & 1.388 & \multicolumn{2}{|c|}{1.928} & 13.331 & 22.164 & 5.24 & 167714 & 1.327 & 1.274 & 16.64 & 2.48 \\
\hline 459 & III & 1.674 & 1.103 & \multicolumn{2}{|c|}{1.218} & 3.548 & 2.569 & 4.37 & 155095 & 1.316 & 1.274 & 2.57 & 1.28 \\
\hline 458 & III & 1.703 & 1.118 & \multicolumn{2}{|c|}{1.251} & 4.847 & 4.849 & 4.37 & 155095 & 1.316 & 1.274 & 5.42 & 1.21 \\
\hline 460 & III & 1.668 & 1.047 & \multicolumn{2}{|c|}{1.097} & 6.607 & 7.257 & 4.37 & 155095 & 1.316 & 1.274 & 7.35 & 1.07 \\
\hline 456 & 1 & & & & & 2.627 & 2.053 & & & & & & \\
\hline 457 & 1 & 2.024 & 1.222 & \multicolumn{2}{|c|}{1.494} & 5.705 & 4.203 & 5.13 & 80916 & 1.360 & 1.393 & 7.04 & 1.60 \\
\hline 494 & 1 & 1.934 & 0.909 & \multicolumn{2}{|c|}{0.826} & 16.392 & 8.260 & 5.13 & 80916 & 1.360 & 1.393 & 9.80 & 2.16 \\
\hline 491 & 1 & 4.301 & 2.268 & \multicolumn{2}{|c|}{5.143} & 7.009 & 7.547 & 4.23 & 71875 & 1.350 & 1.393 & 7.29 & 2.40 \\
\hline 492 & 1 & 6.121 & 3.120 & \multicolumn{2}{|c|}{9.737} & 12.108 & 12.812 & 4.23 & 71875 & 1.350 & 1.393 & 9.54 & 2.55 \\
\hline 493 & 1 & 6.641 & 3.258 & \multicolumn{2}{|c|}{10.614} & 16.918 & 11.752 & 4.23 & 71875 & 1.350 & 1.393 & 12.15 & 2.92 \\
\hline Versuch & Bedingung & $\delta^{+}{ }_{x 1} / L_{1}$ & $\mathrm{M}_{\mathrm{x} 1}^{+}$ & $\mathrm{Re}_{\mathrm{x} 1}^{+}$ & $\mathrm{T}_{\mathrm{x} 1}^{+}$ & $\mathrm{L}_{\text {sep }} / \delta_{\mathrm{x} 1}^{+}$ & $\Delta \mathrm{L}_{\text {sep }} / \delta^{+}{ }_{\mathrm{x} 1}$ & $\operatorname{Re}_{x 1}^{+}{ }^{1 / 2} / M^{+}$ & $\times 1^{3} \quad(R$ & )$^{1 / 2} / \mathrm{M}^{+}{ }^{3}{ }^{3}$ & & $\Lambda_{1}^{3}$ & $\left.{ }_{1}^{1} / \mathrm{C}\right)^{12} / \mathrm{M}_{1}{ }^{3}$ \\
\hline 446 & IIII & & & & & & & & & & & & \\
\hline 455 & III & & & & & & & & & & & & \\
\hline 454 & III & & & & & & & & & & & & \\
\hline 453 & III & 0.00378 & 2.60 & 33458 & 2570 & 115.1 & 4.94 & 10.36 & & 9.18 & & & 2.52 \\
\hline 452 & III & 0.00356 & 2.61 & 29754 & 2584 & 149.3 & 5.35 & 9.68 & & 8.58 & & & 2.52 \\
\hline 447 & III & 0.00309 & 2.62 & 22577 & 2593 & 231.0 & 6.60 & 8.39 & & 7.44 & & & 2.52 \\
\hline 459 & III & 0.00370 & 2.42 & 60179 & 2751 & 50.1 & 5.05 & 17.40 & & 15.41 & & & 4.19 \\
\hline 458 & III & 0.00342 & 2.42 & 51863 & 2759 & 86.5 & 5.38 & 16.13 & & 14.29 & & & 4.19 \\
\hline 460 & III & 0.00325 & 2.42 & 46996 & 2757 & 104.7 & 5.41 & 15.33 & & 13.59 & & & 4.19 \\
\hline 456 & I & & & & & & & & & & & & \\
\hline 457 & I & 0.00598 & 2.58 & 21716 & 3636 & 38.0 & 3.05 & 8.61 & & 7.30 & & & 1.78 \\
\hline 494 & I & 0.00486 & 2.58 & 14552 & 3660 & 117.3 & 4.01 & 7.04 & & 5.97 & & & 1.78 \\
\hline 491 & I & 0.00488 & 2.43 & 26262 & 3929 & 75.7 & 4.08 & 11.29 & & 9.57 & & & 2.99 \\
\hline 492 & I & 0.00439 & 2.43 & 21326 & 3942 & 134.4 & 4.91 & 10.15 & & 8.60 & & & 2.99 \\
\hline 493 & I & 0.00378 & 2.44 & 15776 & 3965 & 182.6 & 6.22 & 8.69 & & 7.36 & & & 2.99 \\
\hline
\end{tabular}

Tabelle 5.8: Charakteristische experimentelle und numerische Werte für die Doppelrampenversuche. 

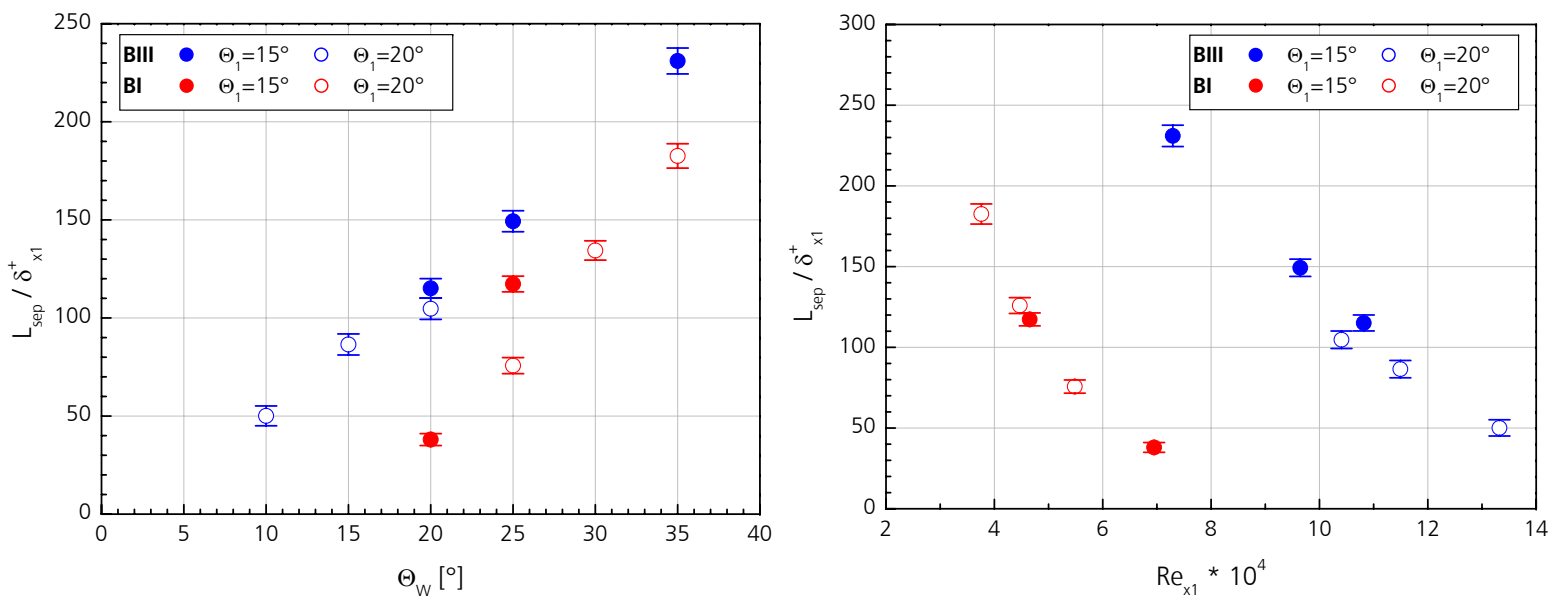

Abbildung 5.30: Länge der Ablösung in Abhängigkeit vom Umlenkwinkel $\Theta_{W}$ der zweiten Rampe (links) und der lokalen Reynoldszahl $R e_{x 1}$ am Rand der Verdrängungsdicke $\delta_{x 1}^{+}$am Ort der Strömungsablösung (rechts). Die Ablöselänge ist mit der Verdrängungsdicke normiert.

ist ein zentrales Ergebnis vieler Studien, dass sich die Ablöselänge mit steigendem Umlenkwinkel vergrößert. In Abb. $\mathbf{5 . 3 0}$ links sind die gemessenen Ablöselängen, normiert mit der Verdrängungsdicke $\delta_{x 1}^{+}$ab Ablöseort, über dem Umlenkwinkel $\Theta_{W}$ der zweiten Rampe aufgetragen. Die Ablöselänge vergrößert sich mit größerem Umlenkwinkel. Die Steigung des Verlaufes hängt allerdings von der benutzten Bedingung und dem Winkel $\Theta_{1}$ der ersten Rampe ab. Der Schnittpunkt der Geraden mit der Abszisse stellt den Winkel, bzw. Drucksprung dar, unter dem die Strömung beginnt abzulösen. Katzer (1989) fand bei seinen Untersuchungen eine Abhängigkeit von der lokalen Machzahl (s. Abschnitt 2.4, Gl. 2.5). Wir kommen weiter unten darauf zurück.

Für rein laminare Strömungen wurde beobachtet, dass sich die Ablöselänge mit steigender lokaler Reynoldszahl am Ort der Ablösung $x 1$ und am Rand der Grenzschichtverdrängungsdicke $\delta_{x 1}^{+}$vergrößert. Diese Abhängigkeit ist in der Abb. 5.30 rechts aufgetragen. Es ist deutlich zu erkennen, dass sich statt dessen für alle untersuchten Fälle die Ablöselänge mit steigender Reynoldszahl $R e_{x 1}$ verkleinert. Dieses Verhalten ist bei solchen Strömungen beobachtet worden, bei denen ein transitioneller Charakter vorliegt. Hunter u. Reeves (1971) fanden diesen Trend bei vollturbulenter Strömung, wenn Relaminarisierung der Strömung hinter der Umlenkung eintrat.

In der Tab. 5.9 sind die Werte für die beginnende Ablösung nach Katzer aufgelistet und die Werte die sich aus der Geradengleichung $\frac{L_{s} e p}{\delta_{x 1}^{+}}=a+b * \Theta_{W}$ der Auftragung in Abb. 5.30 für die HEG-Experimente ergeben.

In der Abb. $\mathbf{5 . 3 1}$ sind diese Druckverhältnisse nach Katzer mit den aus den HEG Experimen-

\begin{tabular}{|c|c|c|c|c|c|c|c|c|c|}
\hline Bedingung & $\Theta_{1}$ & a & b & $\Theta_{\text {inc }}$ & $M_{1}$ & $\kappa_{1}$ & $\mathrm{cf}_{\mathrm{L}}$ & $\mathbf{p}_{\text {ind }} / \mathbf{p}_{1}$ Katzer & $p_{\text {inc }} / p_{1}$ Experiment \\
\hline III & 15 & -42.600 & 7.790 & 5.469 & 5.239 & 1.3270 & 0.1422 & 8.922 & 1.899 \\
\hline III & 20 & -1.460 & 5.460 & 0.267 & 4.368 & 1.3160 & 0.1090 & 6.258 & 1.028 \\
\hline I & 15 & -279.200 & 15.820 & 17.649 & 5.132 & 1.3600 & 0.1355 & 8.688 & 5.914 \\
\hline । & 20 & -192.633 & 10.690 & 18.020 & 4.234 & 1.3500 & 0.0941 & 5.788 & 4.701 \\
\hline
\end{tabular}

Tabelle 5.9: Wertetabelle für die beginnende Ablösung nach Katzer und experimentelle Ergebnisse. 

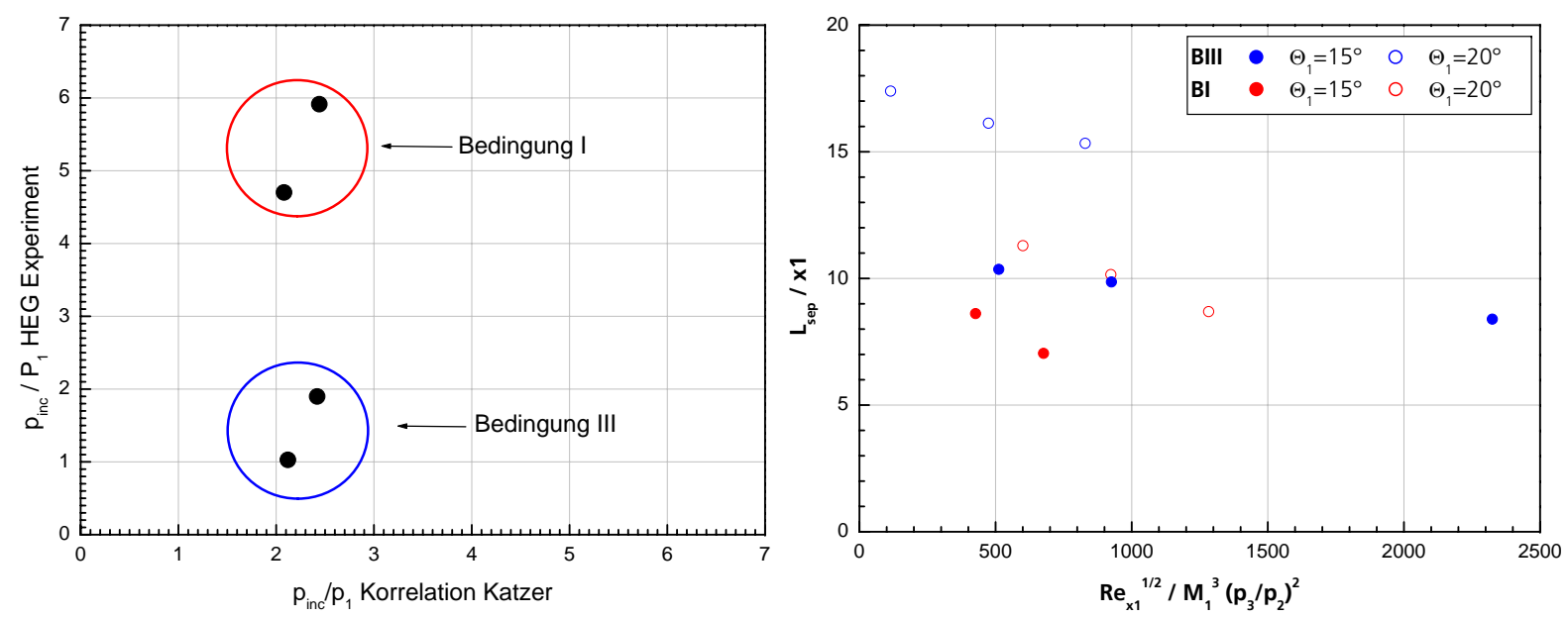

Abbildung 5.31: Druckverhältnis für beginnende Ablösung der HEG-Experimente im Vergleich zu der Korrelation von Katzer (links) und Korrelation von Needham angewandt auf die HEG-Ergebnisse für die Ablöselänge (rechts).

ten ermittelten Werten ins Verhältnis gebracht. Es wird deutlich, dass sich Bedingung I und Bedingung III klar unterscheiden. Bei der Versuchsbedingung III (niedrige Enthalpie) setzt die Ablösung der Strömung eher ein, als es die Korrelation von Katzer vorhersagt. Ganz im Gegensatz hierzu setzt die Ablösung für die Versuchsbedingung I (hohe Enthalpie) sehr viel später ein. Trotz dieses Unterschiedes scheint das Einsetzen der Ablösung der Korrelation von Katzer zu folgen, obwohl diese Korrelation auf Basis von Experimenten für rein laminare Strömungen bestimmt wurde. Die in Abschnitt 2.4 beschriebene Korrelation von Needham bringt das Verhalten der Ablöselänge zu den das Problem bestimmenden Parametern ins Verhältnis. Die für die HEG-Experimente bestimmten Werte sind in der Tab. $\mathbf{5 . 9}$ aufgelistet und in der Abb. 5.31 auf der rechten Seite dargestellt. Das Verhalten der abgelösten Strömung bei den HEG-Experimenten korreliert umgekehrt proportional zu der Korrelation von Needham, auch dies ist ein deutliches Indiz dafür, dass es sich bei der beobachteten abgelösten Strömung um eine transitionelle Strömung handelt.

\begin{tabular}{|c|c|c|c|c|c|c|c|c|}
\hline Versuch & Bedingung & $\Theta_{1}$ & $\Theta_{2}$ & $\Theta_{w}$ & Lsep/x1 & $\operatorname{Re}_{x 1}^{+}{ }^{1 / 2} / \mathrm{M}^{+}{ }^{+}{ }^{3}$ & $\left(p_{3} / p_{2}\right)^{2}$ & Lsep/x1 (Needham) \\
\hline 446 & III & 0 & 0 & 0 & & & & \\
\hline 455 & III & 15 & 25 & 10 & & & & \\
\hline 454 & III & 15 & 30 & 15 & & & & \\
\hline 453 & III & 15 & 35 & 20 & 0.674 & 10.359 & 49.414 & 511.89 \\
\hline 452 & III & 15 & 40 & 25 & 0.924 & 9.680 & 95.636 & 925.71 \\
\hline 447 & III & 15 & 50 & 35 & 1.641 & 8.393 & 276.970 & 2324.62 \\
\hline 459 & III & 20 & 30 & 10 & 0.216 & 17.395 & 6.587 & 114.58 \\
\hline 458 & III & 20 & 35 & 15 & 0.399 & 16.129 & 29.377 & 473.82 \\
\hline 460 & III & 20 & 40 & 20 & 0.507 & 15.334 & 54.069 & 829.10 \\
\hline 456 & 1 & 15 & 25 & 10 & & & & \\
\hline 457 & I & 15 & 35 & 20 & 0.264 & 8.611 & 49.558 & 426.73 \\
\hline 494 & I & 15 & 40 & 25 & 0.992 & 7.041 & 96.030 & 676.11 \\
\hline 491 & 1 & 20 & 45 & 25 & 0.484 & 11.294 & 53.159 & 600.37 \\
\hline 492 & I & 20 & 50 & 30 & 1.011 & 10.152 & 90.959 & 923.45 \\
\hline 493 & I & 20 & 55 & 35 & 1.320 & 8.689 & 147.636 & 1282.80 \\
\hline
\end{tabular}

Tabelle 5.10: Wertetabelle für die Korrelation nach Needham (s. Abschnitt 2.4). 


\subsubsection{Wiederanlegewärmestromdichten}

Die Untersuchungen von Holden (1972) ergaben, dass sich das Verhältnis der maximalen Wärmestromdichte beim Wiederanlegen zur Wärmestromdichte auf der ersten Rampe in direkte Abhängigkeit zum korrespondierenden Druckverhältnis setzen lässt:

$$
\frac{\dot{q}_{p k}}{\dot{q}_{1}}=F \cdot \frac{p_{p k}{ }^{n}}{p_{1}} .
$$

Für vollturbulente Strömungen korrelierte das Wertepaar von $F=1.0 ; n=0.85$ die Daten von Holden (1972) gut, während die Messergebnisse für transitionelle und laminare Strömungen zwischen den Werten $n=0.7$ und $n=0.5$ lagen. Von anderen Autoren wurden wiederum andere Vorschläge gemacht. Innerhalb der ATDB (Aerothermodynamischen Datenbank) des X-38-Projektes ergab das Wertepaar $F=1.0 ; n=1.1$ für turbulente Strömungen und das Wertepaar $F=1.2 ; n=0.8$ für laminare Strömungen die beste Übereinstimmung. In Abb. $\mathbf{5 . 3 2}$ werden die Messungen an der Doppelrampe und die Messungen am X-38-Modell mit dieser Korrelation verglichen.
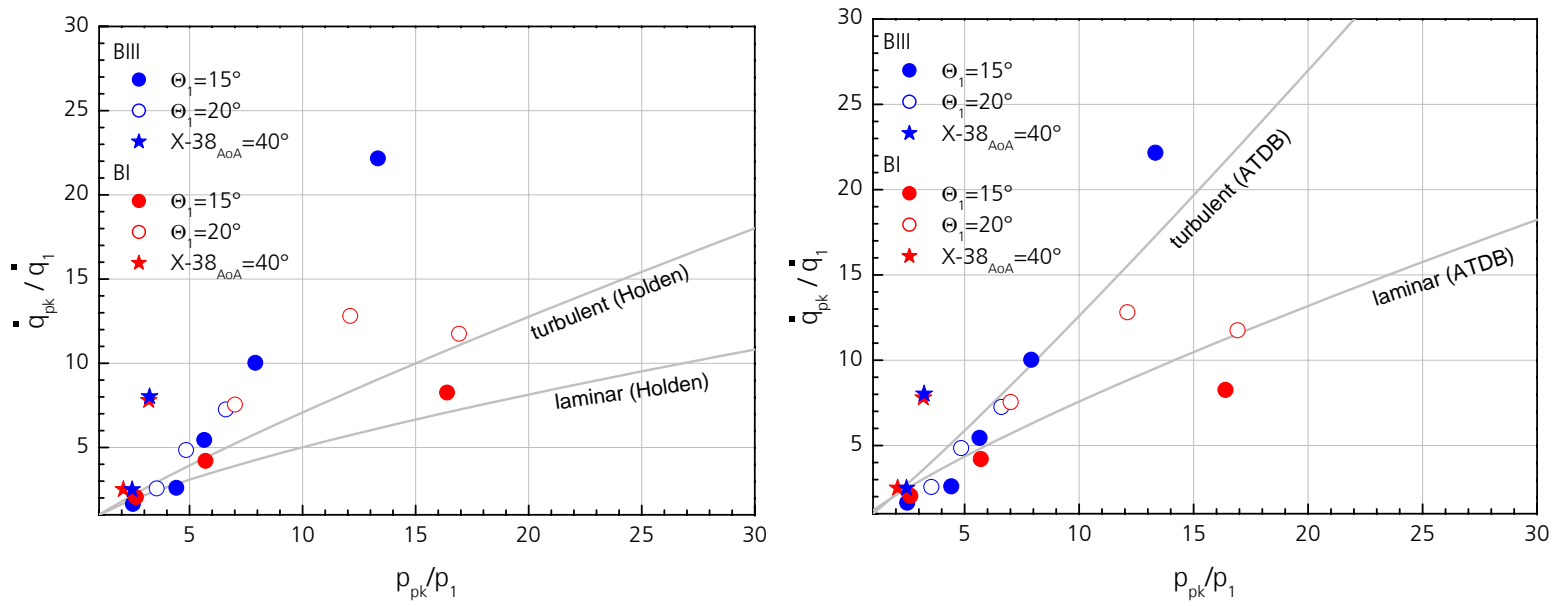

Abbildung 5.32: Wärmstromverhältnis zwischen dem Wiederanlegeort der Strömung und dem Wert auf der ersten Rampe zum korrespondierenden Druckverhältnis. Auf der linken Seite ist die Korrelation von Holden und auf der rechten Seite die Korrelation innerhalb der X-38-ATDB mit dargestellt.

Zusätzlich zu den Ergebnissen der Doppelrampenmessungen sind die Werte für die Messung am X-38-Modell für die Strömungsumlenkung mit der $20^{\circ}$-Klappe und der $30^{\circ}$-Klappe eingetragen. Während die Messungen für die Doppelrampe alle über der Korrelation von Holden (1972) liegen, scheint die innerhalb des X-38-Projektes benutzte Korrelation den Verlauf der Doppelrampenmessungen eher wiederzugeben. Deutlich wird jedoch, dass die Wärmestromdichteerhöhung bei den X-38-Messungen viel größer als bei den Messungen an der Doppelrampe wird. Insgesamt liegt die Mehrzahl der HEG-Versuche zwischen den Grenzen für laminare und voll turbulente Strömungen. 


\section{Zusammenfassung und Ausblick}

In der vorliegenden Arbeit werden die im Hochenthalpiekanal Göttingen (HEG) an einem Windkanalmodell experimentell gewonnenen Messergebnisse für die Wiedereintrittskonfiguration des X-38-Experimentalvehikels diskutiert und mit numerischen Ergebnissen verglichen.

Ein zentrales Ergebnis dieser Untersuchung ist die experimentell gefundene Überhöhung der Wärmestromdichte beim Wiederanlegen der abgelösten Strömung vor den Steuerklappen. Diese Überhöhung konnte von den numerischen Simulationen, die für das Windkanalmodell durchgeführt wurden, nicht rekonstruiert werden. Auch gibt es keine dem Autor bekannte Literaturquelle, die über ein ähnliches Phänomen berichtet. Die gemessenen Wärmeübergangserhöhungen auf den Klappen fallen von der Höhe her in den Bereich transitioneller Strömungen, wie durch eine Transitionsannahme in der numerischen Rechnung gezeigt werden konnte. Die Strömungsbedingungen im HEG weisen sehr niedrige Reynoldszahlen (150000 $\frac{1}{m}-300000 \frac{1}{m}$ ) auf, bei denen sich die abgelöste Strömung laminar verhalten sollte.

Um dieses Ergebnis besser interpretieren und in einen Zusammenhang zu existierenden Untersuchungen bringen zu können, wurden Experimente mit einem querangeströmten Zylinder durchgeführt. Hier stand eine Validierung des benutzen Rechenverfahrens hinsichtlich der Reaktionsratenmodellierung im Vordergrund. Bei den Untersuchungen am Zylinder konnte erstmals gezeigt werden, dass eine Validierung der Reaktionsratenmodellierung mit Hilfe der gemessenen Phasenverschiebungen durch die Technik der holographischen Interferometrie möglich ist. Zusätzlich konnte durch diese Experimente eine Dichtebestimmung der freien Anströmung im HEG durchgeführt werden.

Die Strömungstopologie um die Steuerklappen des Windkanalmodells wurde mit einem generischen zweidimensionalen Doppelrampenmodell experimentell untersucht. Bei diesen Untersuchungen konnten mehr Daten zur Klassifizierung des gefundenen Phänomens beim X-38 gewonnen werden. Durch die eigens entwickelte HochgeschwindigkeitsSichtbarmachung konnte experimentell gezeigt werden, dass sich die Ablöseblase in der zur Verfügung stehenden Messzeit etabliert und konstant verhält. Die experimentellen Daten zeigen, dass sich die abgelöste Strömung in allen untersuchten Fällen transitionell verhält. Der Vergleich der Ablöselänge und des Wärmestromdichte beim Wiederanlegen mit bestehenden Korrelationen stützt diese Aussage. Dieser transitionelle Charakter ist unbeeinflusst von der Ruheenthalpie $h_{0}$ der benutzten Bedingung und auch unabhängig von der verwendeten Reynoldszahl Re. Bei allen Vergleichen mit existierenden Korrelation zeigt sich, dass sich die Größe der Ablöseblase mit höherer Ruheenthalpie $h_{0}$ verringert.

Während sich die abgelöste Strömung für das Doppelrampenmodell immer im Bereich einer Transition zur vollturbulenten Strömung befand, konnten am X-38-Modell auch der Fall einer rein laminaren Strömung erzeugt werden. Da die Vorlaufströmung im ersten Fall durch einen scharfen Keil, im zweiten Fall durch eine stumpfe Nase erzeugt wird, sollte die dieses Phänomen steuernde Ursache hier zu finden sein. Wichtig für zukünftige Untersuchungen ist vor allem die Möglichkeit zur Kombination beider Fälle in einem Modell. Deshalb 
wird für zukünftige Arbeiten auf diesem Gebiet die in Abb. 6.1 dargestellte Modellkonfiguration vorgeschlagen. Sie bietet die Möglichkeit, im Staubereich des Modells optische Untersuchungen durchzuführen während, gleichzeitig Untersuchungen im Bereich der abgelösten Strömung stattfinden können. Durch einfaches Auswechseln der Spitze des Modells können Einflüsse der Vorlaufströmung experimentell dargestellt werden. Durch gleichzeitige Anwendung der holographischen Interferometrie und der HochgeschwindigkeitsSichtbarmachung kann zum einen die zeitliche Entwicklung der Ablösung und zum anderen die Reaktionskinetik in der Vorlaufströmung experimentell untersucht werden, um die Wechselwirkung zwischen beiden genauer zu untersuchen.
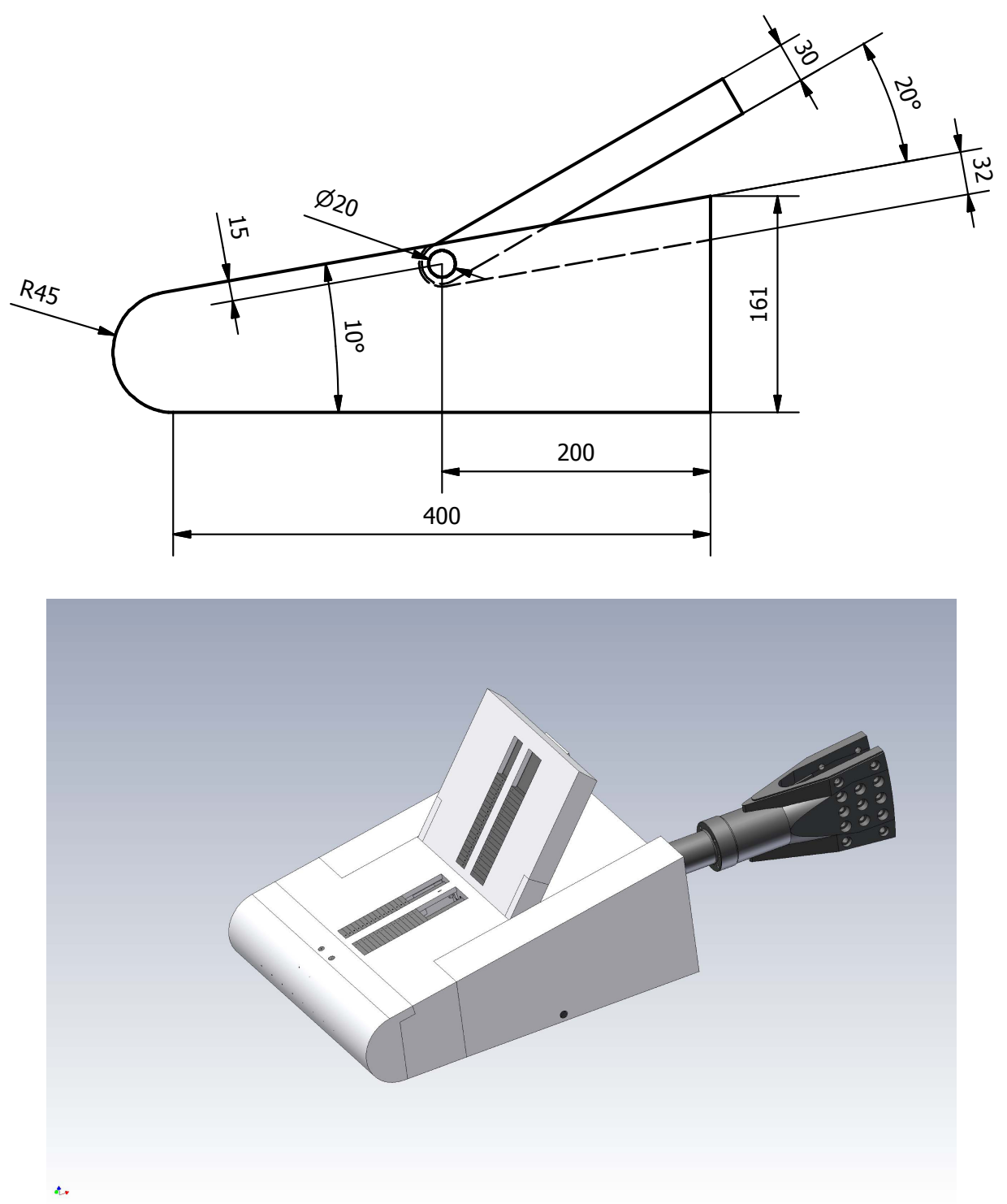

Abbildung 6.1: Vorschlag für den Entwurf eines Doppelrampenmodells, dass die kombinierte Untersuchung der Wechselwirkung zwischen Naseneinfluss und abgelöster Strömung erlaubt. 


\section{Literatur}

[Ackeret u. a. 1946]

ACKERET, J ; FELDMANN, F ; ROtT, N: Untersuchungen an Verdichtungsstößen und Grenzschichten in schnell bewegten Gasen / Institut für Aerodynamik ETH Zürich. 1946 (10). Forschungsbericht. vergl. auch NACA TM 1133 (1947)

[Alpher u. White 1959]

ALPHER, RA ; WHITE, RR: Optical refractivity of high-temperature gases. In: Physics of Fluids 2 (1959), S. 153-161

\section{[Anders 1970]}

ANDERS, JB: Wedge-induced laminar-boundary-layer separation on a flat plate in lowdensity, hypervelocity flow / NASA. 1970 (TN D-5791). - Forschungsbericht

[Anders u. Edwards 1968]

ANDERS, JB ; EDWARDS, CLW: A real-gas study of low-density wedge-induced laminar separation on a high cooled blunt flat plate at $\mathrm{M}_{\infty}=12$ / NASA. 1968 (TN D-4320). Forschungsbericht

[Anderson 1989]

Anderson, JD J. ; BRown, AT (Hrsg.) ; BeAmesderfer, L (Hrsg.) ; MorRiss, JM (Hrsg.): Hypersonic and high temperature gas dynamics. McGraw-Hill, Inc., 1989 (McGraw-Hill Series in aeronautical and aerospace enineering ISBN 0-07-001671-2)

[Asker 1996]

ASKER, JR: For Myriad Woes, NASA Calls X-38 to the Rescue. In: Aviation Week \& Space Techology (1996), Nr. 145(20):70-1

[Asma 2001]

AsmA, CO: Transition and Shock Wave - Boundary Layer Interaction in Hypervelocity Flows, VKI, Diss., june 2001

[ATM 1958]

ATM: U.S. Extension to the ICAO Standard Atmosphere. U.S. Government Printing Office, Washington, D.C., 1958

[ATM 1962]

ATM: U.S. Standard Atmosphere Supplements. U.S. Government Printing Office, Washington, D.C., 1962

[ATM 1966]

ATM: U.S. Standard Atmosphere Supplements. U.S. Government Printing Office, Washington, D.C., 1966 
[ATM 1976]

ATM: U.S. Standard Atmosphere. U.S. Government Printing Office, Washington, D.C., 1976

[Baldwin u. Lomax 1978]

BALDWIN, BS ; LOMAX, H: Thin Layer Approximation and Algebraic Model for Separated Turbulent Flows. In: AIAA Paper AIAA, 1978

[Berry u. a. 2001]

Berry, SA ; Horvath, TJ ; Weilmuenster, KJ ; Alter, SJ ; Merski, NR: X-38 Experimental Aeroheating At Mach 10. (2001), 11 - 14 June, Nr. AIAA Paper 2003-3641. - Anaheim, CA

[Bleiblebens u. Olivier 2006]

BLEIBLEBENS, M ; OLIVIER, H: On the influence of elevated surface temperatures on hypersonic shock wave/boundary layer interaction at a heated ramp model. In: Shock Waves 53 (2006), Nr. 1, S. 149-176

[Blottner u. a. 1971]

BLOTTNER, FG ; JOHNSON, M ; ELLIS, M: Chemically reacting viscous flow program for multi-component gas mixtures / Sandia Laboratories. 1971 (SC-RR-70-745). - Forschungsbericht

[Bloy u. Georgeff 1974]

BLOY, AW ; GEORGEFF, MP: The hypersonic laminar boundary layer near sharp compression and expansion corners. In: Journal of Fluid Mechanics 3 (1974), Nr. 63, S. 431-447

[Bogdonoff u. Kepler 1955]

BOGDONOFF, SM ; KEPLER, CE: Separation of a supersonic turbulent boundary layer. In: Journal of Aerospace Science 22 (1955), S. 414-424

[Brenner u. a. 1993]

Brenner, G; Gernold, T; HANnemanN, K ; Rus, D: Numerical Simulation of Shock/Shock and Shock-Wave/Boundary-Layer Interactions in Hypersonic Flows. In: Computers Fluids 22 (1993), Nr. 4/5, S. 427-439

[Brown 1998]

BRowN, D: Manning the Lifeboats on the International Space Station. In: Launchspace (1998), Nr. 3(2):20-2

[Brown u. a. 1990]

BROWN, SN ; CHENG, HK ; LEE, CJ: Inviscid-viscous interaction on triple-deck scales in a hypersonic flow with strong wall cooling The hypersonic laminar boundary layer near sharp compression and expansion corners. In: Journal of Fluid Mechanics (1990), Nr. 220, S. 309-337

[Brück u. a. 1999]

BRÜCK, S; GIESE, P; HANNEMANN, V; LÜDEKE, H ; ORLOWSKI, M: Contribution of DLR to 
the Aerodynamic and Aerothermodynamic Data Base of the X-38 Vehicle / DLR. 1999. Forschungsbericht. DLR-IB 129-99/12

[Brück u. a. 1995]

BRÜCK, S; RADESPIEL, R ; LONGO, JMA: Comparison of nonequilibrium flows past a simplified Space-Shuttle configuration. In: 77th AGARD Fluid Dynamics Panel Meeting and Symposium on Progress and Changes in CFD Methods and Algorithms AIAA, 1995

[Burggraf 1975]

BURGGRAF, OR: Asymptotic theory of separation and reattachment of a laminar boundary layer on a compression ramp / AGARD. 1975 (CP-168). - Forschungsbericht. In Flow Separation

[Carl u. a. 1998]

CARL, M ; HANnemanN, V; EITElberg, G: Shock/shock interaction experiments in the High Enthalpy Shock Tunnel Göttingen. In: AIAA Paper AIAA, 1998

[Chanetz u. a. 1998]

Chanetz, B ; Benay, R ; Bousquet, JM ; Bur, T ; Grasso, F ; Moss, J: Experimental and Numerical Study of the Laminar Separation in Hypersonic Flow. In: Aerospace Science and Technology (1998), Nr. 3, S. 205-218

[Chavagnac u. a. 2003]

ChavagnaC, C; Moulin, J ; Gerard, Y ; Guedron, S: PRE-X: The first european hypersonic Glider. In: 12th AIAA Space Planes and Hypersonic Systems and Technologies Conference, 2003. - Norfolk Virginia, USA

[Chue u. a. 1998]

ChUe, RSM ; Eitelberg, GM ; CARL, M: Studies of the Transient Flows in High Enthalpy Shock Tunnels. In: Experiments in Fluids 25 (1998), S. 474-486

[Coleman u. Stollery 1972]

COLEMAN, GT ; StOLLERY, JL: Heat transfer from hypersonic turbulent flow at a wedge compression corner. In: Journal of Fluid Mechanics 4 (1972), Nr. 56, S. 741-752

[Cook u. Feldermann 1966]

COOK, WJ ; FeldermanN, EJ: Reduction of Data from Thin-Film Heat Transfer Gages: A Concise Numerical Technique. In: AIAA Journal, Technical Notes 4 (1966), Nr. 3, S. $561-562$

[Covault 1998]

Covault, C: Second X-38 Set for Flight. In: Aviation Week \& Space Techology (1998), Nr. 149(9):58

[Curle 1961]

CURLE, N: The effects of heat transfer on laminar-boundary-layer separation in supersonic flow. In: The Aeronautical Quarterly (1961), Nr. 12, S. 309-336 
[Dändliker u. Thalmann 1985]

DÄNDLIKER, R ; THALMANN, R: Heterodyne and quasi-heterodyne holographic interferometry. In: Optical Engineering (1985), Nr. 24 (5), S. 824-831

[Davis 1996]

DAVIS, J: Further tests on the HALIS axisymmetric configuration in the T5 hypervelocity shock tunnel / GALCIT. 1996 (FM 96-2). - Forschungsbericht

[Davis 1999]

DAVIS, J: High-Enthalpy Shock/Boundary-Layer Interaction on a Double Wedge, California Institute of Technology, Diss., 1999. - submitted September 29, 1998

[Davis u. Sturtevant 1997]

DAVIS, J ; STURTEVANT, B: High enthalpy shock-boundary layer interaction on a double wedge: separation length. In: 21th International Symposium on Shock Waves, 1997. Great Keppel Island, Australia

[Davis u. Sturtevant 2000]

DAVIS, JP ; STURTEVANT, B: Separation length in high-enthalpy shock/boundary-layer interaction. In: Physics of Fluids 12 (2000), Nr. 10, S. 2661-2687

[Debrestian u. Anderson 1993]

DebrestiAn, DJ ; ANDERSON, JD: Reference Temperature Method and Reynolds Analogy for Chemicallly Reacting Nonequilibrium Flowfields. In: Thermophysics 8 (1993), Nr. 1, S. 190-192

[Delery u. Marvin 1975]

DELERY, J ; MARVIN, JG: Shock-wave boundary layer interactions / AGARD. 1975 (AG280). - Forschungsbericht

[Dunn u. Kang 1973]

DUNN, MG ; KANG, SW: Theoretical and experimental studies of reentry plasmas / NASA. 1973 (CR-2232). - Forschungsbericht

[Eckert 1955]

ECKERT, ERG: Engineering Relations for Friction and Heat Transfer to Surfaces in High Velocity Flow. In: Journal of the Aeronautical Sciences (1955), August, S. 585-586

[Edney 1968]

EDNEY, B: Anomalous Heat Transfer and Pressure Distributions on Blunt Bodies at Hypersonic Speeds in the Presence of an Impigning Shock / Aeronautical Research Institute of Sweden. 1968. - FFA Report 115

[Eggers u. a. 2006]

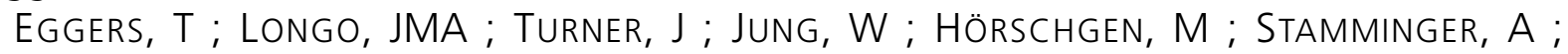
Gülhan, A ; Siebe, F ; Requardt, G ; LAuX, T ; ReImer, T ; Weihs, H: The SHEFEX Flight Experiment - Pathfinder Experiment for a Sky Based Test Facility -. In: 14th AIAA/AHI Space Planes and Hypersonic Systems and Technologies Conference, 2006. - Canberra Australia 
[von Eggers Rudd u. a. 1998]

EgGeRs Rudd, L von ; RANKInS, F ; PINES, DJ: Moveable Cowl Control for Increased Hypersonic Performence. In: AIAA 8th International Space Planes and Hypersonics and Technologies Conference, 1998. - Norfolk, VA, USA

[Elfstrom 1972]

ELFSTROM, GM: Turbulent hypersonic flow at a wedge-compression corner. In: Journal of Fluid Mechanics 1 (1972), Nr. 53, S. 113-127

[Fay u. Ridell 1958]

FAY, JA ; RIDELL, FR: Theory of stagnation point heat transfer in dissociated air. In: Journal of the Aeronautical Sciences 25 (1958), Nr. 2, S. 73-85

[Ferguson u. Schaefer 1962]

FERGUSON, H ; SCHAEFER, JW: Heat transfer and pressure distribution on cone-cylinderflare configuration with boundary-layer separation / NASA. 1962 (TN D-1436). - Forschungsbericht

[Foucault 1859]

FouCAULT, L: Mémoire sur la construction des télescopes en verre argenté. In: Annales de l'Observatoire Impérial de Paris (1859), Nr. 5, S. 197-237

[Gadd u. a. 1954]

GADD, GE ; HOLDER, DW ; REGAN, JD: An experimental investigation of the interaction between shock waves and boundary layers. In: Proceeding of the Royal Society of London, 1954

[Gardner u. a. 2004]

GARDNER, AD ; JACOBS, PA ; HANNEMANN, K: New Results in Numerical and Experimental Fluid Mechanics IV. Bd. 87: End-to-End modelling and design of a new operating condition for HEG. Springer Verlag, 2004

[Georgeff 1974]

GEORGEFF, MP: Monentum integral method for viscous-inviscid interactions with arbitrary wall cooling. In: AIAA Journal 10 (1974), Nr. 12, S. 1393-1400

[Gersten 1998]

GeRSTEN, G: Grenzschichttheorie. Springer Verlag, 1998

[Glick 1962]

GLICK, HS: Modified Crocco-Lees mixing theory for supersonic seperated and reattaching flows. In: Journal of the Aeronautical Sciences 10 (1962), Nr. 29, S. 1238-1249

[Grasso u. Marini 1996]

GRASSO, F ; MARINI, M: Analysis of hypersonic shock-wave laminar boundary-layer interaction phenomena. In: Computers and Fluids 6 (1996), Nr. 25, S. 561-58193 
[Griffith u. a. 1983]

GRIFFITH, BJ ; MAUS, JR ; BEST, JT: Explanation of the hypersonic longitudinal stability problem - lessons learned / NASA. 1983 (CP-2283). - Shuttle Performace: Lessons Learned,

Part 1. Langley, VA, USA

[Gupta u. a. 1990]

GUPTA, RN ; YOSS, JM ; THOMPSON, RA ; LEE, KP: A review of reaction rates and thermodynamic transport properties for an 11-species air model for chemical and thermal nonequilibrium calculations to $30000 \mathrm{~K} /$ NASA. 1990 (RP-1232). - Forschungsbericht

[Hannemann 2003]

HANNEMANN, K: High Enthalpy Flows in the HEG Shock Tunnel: Experiment and Numerical Rebuilding. In: 41st AIAA Aerospace Sciences Meeting and Exhibit, 2003

[Hannemann u. a. 1999]

HanNemann, K; HANNemann, V ; BRÜCK, S ; RAdespiel, R ; SARMA, GSR: Computational Modelling for High Enthalpy Flows. In: SAMA 34 (1999), S. 84-93

[Hannemann u. a. 2001]

HANNEMANn, K; MARTINez SChramm, J ; BRÜECK, S; LONGO, JMA: High Enthalpy Testing and CFD Rebuilding of X-38 in HEG. In: Proc. 23rd International Symposium on Shock Waves, 2001. - Fort Worth, USA

[Hannemann u. a. 2002]

Hannemann, K; Martinez SChramm, J; KarL, S; BeCK, W: Cylinder Shock Layer Density Profiles Measured in High Enthalpy Flows in HEG. In: AIAA Paper 2002-2913, 2002

[Hannemann u. a. 2000]

Hannemann, $\mathrm{K}$; Schnieder, $\mathrm{M}$; Reimann, B ; J, Martinez S.: The Influence and the Delay of Driver Gas Contamination in HEG. In: 21st AIAA Aerodynamic Measurement Technology and Ground Testing Conference, 2000

[Harvey 1968]

HARVEY, WD: Experimental investigationof laminar-flow separation on a flat plate induced by deflected trailing-edge flap at Mach 19 / NASA. 1968 (TN D-4671). - Forschungsbericht

[Hayakawa u. Squire 1982]

HAYAKAWA, K; SQUIRE, LC: The effect of the upstream boundary-layer state on the shock interaction at a compression corner. In: Journal of Fluid Mechanics (1982), Nr. 122, S. 369-394

[Hirschel 2005]

HIRSCHEL, EH: Basics of Aerothermodynamics. Springer-Verlag Berlin Heidelberg New York, 2005 ( ISBN 3-540-22132-8). - Jointly published with the American Insitute of Aeronautics and Astronautics (AIAA) 


\section{[Holden 1978]}

HOLDEN, M: A study of flow separation in regions of shock wave-boundary layer interaction in hypersonic flow. In: 11 th Fluid and Plasma Dynamics Conference, 1978. - Seattle, WA, USA

\section{[Holden 1971]}

HOLDEN, MS: Boundary-layer displacement and leading-edge bluntness effects on attached and separated laminar boundary layers in a compression corner. Part II: Experimental study. In: AIAA Journal 1 (1971), Nr. 9, S. 84-93

\section{[Holden 1972]}

HOLDEN, MS: Shock wave-turbulent boundary layer interaction in hypersonic flow. In: 10 th Aerospace Sciences Meeting, 1972. - San Diego, CA, USA

\section{[Holden 2003]}

HOLDEN, MS: A review of experimental studies for DSMC and Navier-Stokes code validation in laminar regions of shock/shock and shock/boundary layer interation including real gas effects in hypervelocity flows. (2003), 23 - 26 June, Nr. AIAA Paper 2003-3641. - check!

\section{[Holder u. North 1963]}

Holder, DW ; NoRTH, RJ: Schlieren Methods. In: Notes on Applied Science (1963), Nr. 31. - Her Majesty's Stationary Office, London

\section{[Hooker 1961]}

HOOKER, WJ: Interpretation of Heat Gauge Records in Shock Tube Flows. In: Physics of Fluids 4 (1961), Nr. 6, S. 783-784

\section{[Hornung u. a. 1991]}

Hornung, H ; Sturtevant, B ; Bélanger, J ; Sanderson, S; Brouillette, M: Performance Data of the new free-piston shock tunnel T5 at CALCIT. In: Proceedings of the 18th International Symposium on Shock Waves, 1991. - Sendai, Japan

\section{[Hornung 1972]}

HORNUNG, HG: Non-equilibrium dissociating nitrogen flow over spheres and circular cylinders. In: Journal of Fluid Mechanics 53 (1972), Nr. 1, S. 149-176

\section{[Hornung 1987]}

HORNUNG, HG: Comparison of the performace of two types of high-enthalpy facilities for real gas simulation / DLR. 1987 (IB 222 - 87 A 12). - DFVLR-AVA Bericht. Göttingen, Germany

\section{[Hornung 1993]}

HoRNUNG, HG: Experimental Hypervelocity Flow Simulation, Needs, Achievements and Limitations. In: First Pacific International Conference on Aerospace Science and Technology, 1993. - Tainan, Taiwan 
[Hornung 1998]

HORNUNG, HG: 28th Lanchester Memorial Lecture - Experimental real-gas hypersonics. In: Aeronautical Journal 92 (1998), Nr. 920, S. 379-389

[Hornung u. a. 2002]

HoRnung, HG ; AdAm, P; Germain, P ; FujII, K ; RASheEd, A: On Transition and Transition Control in Hypervelocity Flow. In: 9th Asian Congress of Fluid Mechanics, 2002. - Isfahan, Iran

[Hornung u. Wen 1995]

HORNUNG, HG ; WeN, CY: Nonequilibrium dissociating flow over spheres. In: 33rd AIAA Aerospace Sciences Meeting and Exhibit, 1995. - Reno, NV , USA

[Horvath u. a. 2000]

Horvath, TJ ; BerRy, SA ; MerSkI, NR ; FITZGERALD, SM: X-38 Experimental Aerothermodynamics. In: 34th AIAA Thermophysics Conference, 2000. - Denver, CO, USA

[Houwing u. a. 2001]

HOUWING, AFP ; SMITH, DR ; FOX, JS ; DANEHY, PM ; MUDFORD, NR: Laminar boundary layer separation at a fin-body junction in a hypersonic flow. In: Shock Waves 11 (2001), S. $31-42$

[Houwing 1999]

HOUWING, F: Automated interactive analysis of finite fringe interferograms, including deconvolution of data from axisymmetric flows / Institute of Fluid Science, Tohoku University, Sendai, Japan. 1999. - Internal Report

[Hu u. Zhong 1998]

$\mathrm{HU}, \mathrm{SH}$; ZHONG, X: Hypersonic Boundary-Layer Stability over Blunt Leading Edges with Bow-Shock Effects. In: 36th AIAA Aerospace Sciences Meeting and Exhibit, 1998. Reno, NV , USA

[Hunter U. Reeves 1971]

HUNTER, Jr L. ; REEVES, BL: Results of a strong interaction, wake-like model of supersonic separated and reattaching turbulent flows. In: AIAA Journal 14 (1971), Nr. 4, S. 475-481

[Hurle 1971]

HURLE, IR: Nonequilibrium flows with special reference to the nozzle-flow problem. In: Proceedings of the 8th International Shock Tube Symposium, 1971

[Ikawa 1979]

IKAWA, H: Real Gas Laminar Boundary Layer Separation Methodology as Applied to Orbiter Control Surface Effectiveness Prediction. In: 17th Aerospace Sciences Meeting, 1979. - New Orleans, LA

[Inger 1995]

INGER, GR: Nonequilibrium-Dissociated Stagnation Boundary-Layer Flow on an Arbitrarily Catalytic Swept Wing. In: AIAA journal 33 (1995), October, Nr. 10 
[Inger 1996]

INGER, GR: A Theory of Local Heat Transfer in Shock/Laminar Boundary Layer Interactions. In: 27th AIAA Fluid Dynamics Conference. Iowa State University, June 17-20 1996. - New Orleans, LA, USA

[Inger 1998]

INGER, GR: Shock-Viscous Interaction Heating in Nonequilibrium-Dissociated Flows along Arbitrarily-Catalytic Surfaces. In: 29th AIAA Fluid Dynamics Conference AIAA, 1998. Albuquerque, NM

[Inger 2002]

INGER, GR: Non-Equlibrium Boundary Layer Effects on the Aerodynamic Heating of Hypersonic Vehicles. In: Acta Astronautica 36 (2002), Nr. 4, S. 205-216

[Inger u. Elder 1991]

INGER, GR ; ELDER, J: Recombination-Dominated Nonequilibrium Heat Transfer to Arbitrarily Catalytic Hypersonic Vehicles. In: Thermophysics 5 (1991), Nr. 4, S. 449-455

[Inger u. a. 2002]

INGER, GR ; HIGGINS, C ; MORGAN, R: Generalized Nonequilibrium Binary Scaling for Shock Standoff on Hypersonic Blunt Bodies. In: Thermophysics 17 (2002), Nr. 1, S. 126128

[Jacobs 1999]

JACOBS, PA: Shock Tube Modelling iwth L1d / Department of Mechanical Engineering, The University of Queensland. 1999. - Forschungsbericht. Research Report 13/98

[Jahoda u. a. 1967]

JAHODA, FC ; JEFFRIES, RA ; SAWYER, GA: Fractional-fringe holographic plasma interferometry. In: Applied Optics (1967), Nr. 6, S. 1407-1410

[Jessen 1993]

JeSSEN, C: Messung von Druck, Temperatur und Kraft an Modellen im Stoßwellenkanal, RWTH Aachen, Diss., 1993. - Verlag Main, Wissenschaftverlag, Aachen, ISBN 3-92571499-5

[Johnson 1968]

JOHNSON, CB: Pressure and flow-field study at Mach number 8 of flow separation on a flat plate with deflected trailing-edge flap / NASA. 1968 (TN D-4308). - Forschungsbericht

[Jordan 2002]

JORDAN, SA: Investigation of the cylinder separated shear-layer physics by large-eddy simulation. In: International Journal of Heat and Fluid Flow 23 (2002), S. 1-12

[Kandebo 1998]

Kandebo, SW: Vista F-16 Tests Preview X-38 Flight Controls. In: Aviation Week \& Space Techology (1998), Nr. 149(16):41 
[Kaneko u. a. 2002]

KANEKO, M ; MENSHOV, I ; NAKAMURA, Y: Computation of nozzle starting process with thermal and chemical nonequilibrium in high-enthalpy shock tunnel. In: 40th AIAA Aerospace Sciences Meeting and Exhibit, 2002. - Reno, NV , USA

[Karl u. a. 2003]

KARL, S; MARTInez SChramm, J; HANNEMAnN, K: High Enthalpy Cylinder Flow in HEG: A Basis for CFD Validation. In: AIAA Paper 2003-4252, 2003

[Kastell 1997]

KASTELL, D: Aerodynamik eines stumpfen Kegels in reagierender Überschallströmung / Institut für Strömungsmechanik, Deutsche Forschungsanstalt für Luft- und Raumfahrt e.V. Göttingen, 1997 (97-06). - DLR-FB

[Kastell u. a. 1996]

KASTELL, D ; CARL, M ; EITELBERG, G: A combined holographic interferometer and laserschlieren system applied to high temperature high velocity cylinder flow. In: Experiments in Fluids 1 (1996), Nr. 22, S. 57-66

[Katzer 1989]

KATZER, E: On the lengthscales of laminar shock/boundary-layer interation. In: Journal of Fluid Mechanics (1989), Nr. 206, S. 477-496

[Kaye u. Laby 1986]

KAYE, GWC ; LABY, TH: Tables of physical and chemical constants and some mathematical functions. 15th ed edition. New York : Longman Sc. and Tech., 1986. - ISBN 0582463548

[Kidd u. a. 1994]

KIDD, CT ; NELSON, CG ; SCHOTT, WTR: Extraneous thermoelectric emf effects resulting from the press-fit installation of coaxial thermocouples in metal models / AEDC (Arnold Engineering Development Center). Tenesse, USA, 1994 (R-376). - Technical report

[Kindl u. Olivier 1995]

KINDL, H ; OLIVIER, H: Development of a static pressure probe (progress report) / RWTH Aachen. 1995 (HT-PR-E34-711-RWTH). - Forschungsbericht

[Kindl u. Olivier 1996]

KINDL, H ; OLIVIER, H: Development of a static pressure probe (final report) / RWTH Aachen. 1996 (HT-SR-E34-711-RWTH). - Forschungsbericht

[Korkegi 1975]

KORKEGI, RH: Comparison of shock-induced two- and three-dimensional incipient turbulent separation. In: AIAA journal 13 (1975), Nr. 4, S. 534-535

[Körner u. Radespiel 1998]

KÖRNER, H ; RADESPIEL, R: Some recent advances in aerodynamics at DLR. In: 36th AIAA Aerospace Sciences Meeting and Exhibit, 1998. - Reno, NV , USA 
[Korolev u. a. ]

KOROLEV, GL; GAJJAR, JSB ; RUBAN, Al: Once again on the Supersonic Flow Separation near a Corner. In: Journal of Fluid Mechanics

[Krek u. a. 1996]

KReK, R ; BeCK, W; EitelberG, G: Hyperboloid flare experiments in the HEG. In: Proceedings of the 20th International Symposium on Shock Waves, 1996. - Pasadena, CA, USA

[Krek u. Jacobs 1993]

KREK, RM ; JACOBS, PA: STN, Shock tube and nozzle calculations for equilibrium air / The University of Queensland. 1993 (2/93). - Department of Mechanical Engineering Report

[KULITE 2004]

KULITE: Pressure Transducer Handbook / Kulite. 2004. - PDF-Document. erhältlich bei www.kulite.com

[Kumar u. Stollery 1994]

KUMAR, D ; StOlLERY, JL: Hypersonic control flap effectiveness. In: 19 th ICAS Congress, 1994

[Kumar u. Stollery 1996]

KUMAR, D ; STOLLERY, JL: The effects of shear layer transition and leading edge bluntness on control flap effectiveness. In: Proceeding of the 20th International Symposium, 1996.

- Singapore: World Scientific, Pasadena, CA , USA

[Labbe u. a. 1999]

LABbe, SG ; Perez, LF ; Fitzgerald, SM ; Longo, JMA ; Rapuc, M: X-38 NASA/DLR/ESADassault Aviation Integrated Aerodynamic and Aerothermodynamics Activities. In: Atmospheric Reentry Vehicle and Systems Symposium, 1999. - Arcachon, France

[Lemieux u. Hornung 2002]

LEMIEUX, P ; HORNUNG, HG: Development and application of streakline visualization in hypervelocity flows. In: Experiments in Fluids (2002), May, S. 188-195

[Lewis u. a. 1968]

LEWIS, JE ; KUBOTA ; LEES, L: Experimental investigation of supersonic laminar, twodimensional boundary-layer separation in a compression corner with and without cooling. In: AIAA Journal 1 (1968), Nr. 6, S. 7-14

[Liepmann 1946]

LIEPMANN, HW: The interaction between boundary layer and shock waves in transonic flow. In: Journal of Aerospace Science 13 (1946), S. 623-637

[Liepmann u. Roshko 1957]

LiePMANN, HW ; Roshko, A: Elements of Gasdynamics. Dover Publications, Inc., 1957 (Originally published: New York: Wiley, c1957, in series: Galcit aeronautical series ISBN 0-486-41963-0) 
[Loomis u. a. 1997]

LoOMis, MP ; Venkatapathy, E ; PApadopoulos, P ; DaVIes, CB ; Berry, S; Horvath, T ; CAMPBELL, C: Aeroheating and Aerodynamic CFD Validation and Prediction for the $X-38$ Program. In: 32nd Thermophysics Conference, 1997. - Atlanta, GA, USA

[Mallinson u. Gai 1992]

MALLINSON, SG ; GAI, SL: Establishment of Laminar Separated Flows in a Free-Piston Shock Tunnel. In: IUTAM Symposium: Aerothermochemistry of Spacecraft and Associated Hypersonic Flows, 1992. - Universite de Provence, Marseille, France

[Mallinson u. a. 1992]

MALLINSON, SG ; GAI, SL ; MUdFORD, NR: Laminar Separated Flows of High Enthalpy Air in a Compression Corner. In: 11th Australasian Fluid Mechanics Conference, 1992. University of Tasmania, Hobart, Tasmania

[Mallinson u. a. 1995a]

MALLINSON, SG ; GAI, SL ; MUdfORd, NR: Compression Corner Flow of an Equilibrium Dissociating Gas / Department of Aerospace and Mechanical Engineering, University College, University of New South Wales, Australian Defence Force Academy. 1995. Forschungsbericht. Aerospace Technical Report 95-1

[Mallinson u. a. 1995b]

MALLINSON, SG ; GAI, SL ; MUdFORD, NR: High enthalpy, hypersonic compression corner flow. In: 33rd Aerospace Sciences Meeting and Exhibit, 1995. - Reno, NV, USA

[Mallinson u. a. 1995c]

MALLINSON, SG ; GAI, SL ; MUdFORD, NR: Leading edge bluntness effects in hypervelocity compression corner flow. In: 20th International Symposium on Shock Waves, 1995. Pasadana, CA, USA

[Mallinson u. a. 1996a]

MALLINSON, SG ; GAI, SL ; MUdFORD, NR: High-Entalphy, Hypersonic Compression Corner Flow. In: AIAA journal 34 (1996), Nr. 6, S. 1130-1137

[Mallinson u. a. 1996b]

MALLINSON, SG; GAI, SL; MUdFORD, NR: Upstream influence and peak heating in hypervelocity shock wave/boundary-layer interaction. In: Journal of Propulsion and Power 5 (1996), Nr. 12, S. 984-990

[Mallinson u. a. 1997a]

MALLINSON, SG ; GAI, SL ; MUdFORD, NR: The interaction of a shock wave with a laminar boundary layer at a compression corner in high-enthalpy flows including real gas effects. In: Journal of Fluid Mechanics 342 (1997), S. 1-35

[Mallinson u. a. 1997b]

MALLINSON, SG ; GAI, SL ; MUDFORD, NR: The interaction ofa shock wave with a laminar boundary layer at a compression corner in high-entalphy flows including real gas effects. In: Journal of Fluid Mechanics (1997), Nr. 342, S. 1-35 


\section{[Mallison 1994]}

MALLISON, SG: Shock Wave / Boundary layer Interaction at a Compression Corner in Hypervelocity Flows, University of New South Wales, Diss., 1994

[Manders 1970]

MANDERS, R: Apollo 11 entry posflight analysis / NASA. 1970 (70-FM-30). - MSC Internal Note

[Martinez Schramm 1999]

MARTINEZ SCHRAMM, G: Shock Boundary Layer Interaction in Hypersonic High Enthalpy Flow on a Double Wedge. In: 22nd International Symposium on Shock Waves, 1999. London, United Kingdom

[Martinez Schramm u. a. 2004a]

Martinez Schramm, J ; Boutry, A ; Vital Durand, M; Hannemann, K: Time Resolved Holographic Interferometry for Short Duration Hypersonic High Enthalpy Facilities. In: 24th International Symposium on Shock Waves, 2004. - Beijing

[Martinez Schramm u. a. 2001]

MARTINeZ SCHRAMM, J; HANNEMANN, K ; BRÜECK, S: High Enthalpy Testing and CFD Rebuilding of X-38 in HEG / German Aerospace Center, DLR. 2001 (TET-DLR-21-TN3103). - TETRA, Technical Report

[Martinez Schramm u. a. 2004b]

MARTinez SChRAMm, J; KARL, S; HANnEMAnN, K.: High Speed Flow Visualization at HEG. In: Breitsamter (Hrsg.) ; LASCHKa (Hrsg.); HeInemann (Hrsg.) ; SPRinger (Hrsg.): New Results in Numerical and Experimental Fluid Mechanics IV Bd. 87. Springer, 2004, S. 229-235

[Maus u. a. 1984]

MAUS, JR ; GRIFFITH, BJ ; SZEMA, KY: Hypersonic Mach number and real gas effects on space shuttle orbiter aerodynamics. In: Journal of Spacecraft and Rockets 21 (1984), Nr. 2, S. 136-141

[Mclntosh 1968]

MCINTOSH, MK: Computer Program for the Numerical Calculation of Frozen and Equilibrium Conditions in Shock Tunnels / Department of Physics,Australian National University. 1968. - Forschungsbericht. Canberra A.C.T.

[Merzkirch 1987]

MERZKIRCH, W ; (Hrsg.): Flow Visualization. Academic Press, 1987 (- I-)

[Miller u. a. 1964]

MILLER, DS ; HIJMAN, R ; CHILDS, ME: Mach 8 to 22 studies of flow separations due to deflected control surfaces. In: AIAA Journal 2 (1964), Nr. 2, S. 312-321

[Millikan u. White 1963]

MILLIKAN, RC ; WHITE, DR: Systematics of Vibrational Relaxation. In: Journal of Chem. Physics 39 (1963), Nr. 12 
[Morring 2004]

MorRING, F: Next Up: Selection fo Orion contractor starts the clock on shrinking the gap in the U.S. access to space. In: Aviation Week \& Space Techology (2004), Nr. (4):22-23

[Moss 1998]

Moss, JN: Shock-Wave/Boundary-Layer Interactions in Hypersonic Low Density Flows. In: 7th AIAAIASME Joint Thermophysics and Heat Transfer Conference, 1998. - Albuquerque, NM, USA

[Muratore u. a. 2000]

Muratore, JF ; Labbe, SG ; Perez, LF ; Thirkettle, A ; Belmont, JP ; Longo, JMA ; MolinA, R: X-38 Aerodynamic Design Data Book / NASA. 2000 (EG3-X38-ADB0001, Revision No.: A,B). - JSC-28854

[NACA1135 1953]

NACA 1135: Equation, tables and charts for compressible flow / National Advisory Commitee for Aeronautics. 1953 (1135). - Forschungsbericht

[Navarro-Martinez u. a. 2001]

NAVArRo-Martinez, S; TUtTy, OR ; Roberts, GT: Numerical Results over Two and Three Dimensional Hypersonic Compression Ramps. In: 23rd International Symposium on Shock Waves, 2001. - Dallas, Texas, USA

[Needham 1965]

NEEDHAM, DA: Laminar Separation in Hypersonic Flow, University of London, Diss., 1965. - Ph.D.thesis

[Needham u. Stollery 1966]

NeEdHAM, DA ; StOlLeRY, JL: Boundary layer separation in hypersonic flow. In: 4 th Aerospace Sciences Meeting, 1966. - Los Angeles, CA, USA

[Neumann 1994]

NeUmAnN, RD: CFD Code Validation - an Instrumentation Perspective. In: 18th AIAA Aerospace Ground Testing Conference, 1994. - Colorado Spring, CO, USA

[Nishida u. a. 1999]

NISHIDA, M ; IDETA, T; KUCHI-ISHI, S; TAKEISHI, K: Numericalstudies of supersonic cavity flows of high-temperature dissociated. In: 22nd International Symposium on Shock Waves, 1999. - Imperial College, London, UK

[Oertel 1994]

OERTEL, H J.: Aerothermodynamik. Springer-Verlag Berlin Heidelberg New York, 1994 ( ISBN 3-540-57008-X)

[Olejniczak u. Candler 1998]

OLEJNICZAK, J ; CANDLER, GV: Computation of Hypersonic Shock Interaction Flow Fields. In: 7th AIAA/ASME Joint Thermophysics and Heat Transfer Conference, 1998. - Albuquerque, NM, USA 
[Oleyniczak u. a. 1997]

OLEYNICZAK, J ; WRIGHT, MJ ; CANDLER, GV: Numerical study of inviscid shock interactions on double-wedge geometries. In: Journal of Fluid Mechanics (1997), Nr. 352, S. 1-25

[Olivier u. a. 1995]

Olivier, H ; GRÖNIG, H ; Le BOzeC, A: Hypersonic Model Testing in a Shock Tunnel. In: AlAA journal 33 (1995), February, Nr. 2

[Park 1985]

PARK, C: On Convergence of Computation of Chemically Reacting Flows. In: 23rd Aerospace Sciences Meeting AIAA, 1985. - Reno, NV, USA

[Park 1999]

PARK, C: Interaction of spalled particles with shock layer flow. In: 37th AIAA Aerospace Sciences Meeting and Exhibit, 1999. - Reno, NV , USA

[Paull u. a. 2002]

PAULL, A ; AlESI, H ; ANDERSON, S: The HyShot Flight Program and how it was developed. In: AIAA/AAAF 11 th International Space Planes and Hypersonic Systems and Technologies Conference AIAA, 2002. - Orléans, France

[Press u. a. 1992]

Press, WH ; Teukolsky, SA ; VetTerling, WT ; Flannery, BP ; (Hrsg.): Numerical Recipes in C. Cambridge University Press, 1992. - ISBN -

[Prince u. a. 1999]

Prince, SA ; VAnNahme, M ; Stollery, JL: Experiments on the Hypersonic Turbulent Shockwave/Boundary Layer Interaction and the Effects of Surface Roughness. In: 37th AIAA Aerospace Sciences Meeting and Exhibit, 1999. - Reno, NV , USA

[Radespiel u. a. 1995]

RADESPIEL, R ; LONGO, JMA ; BRÜUCK, S; SCHWAMBORN, D: Efficient Numerical Simulation of Complex 3D Flows with Large Contrast. In: 77th AGARD Fluid Dynamics Panel Meeting and Symposium on Progress and Changes in CFD Methods and Algorithms AGARD, 1995, S. 33-1 - 33-11. - Sevilla

[Reimann 2000]

REIMANN, B: Zeitaufgelöste Strömungssichtbarmachung von Stoß-StoßWechselweikungen in Hochenthalpieströmungen / Deutsches Zentrum für Luftund Raumfahrt e.V. 2000 (DLR-IB 223-2000 A 07). - Forschungsbericht

[Roshko u. Thomke 1976]

RoshKo, A ; THOMKE, GJ: Flare-induced interaction lenghths in supersonic, turbulent boundary layers. In: AIAA Journal 7 (1976), Nr. 14, S. 873-879

[Ruban u. Turkyilmaz ]

RubAN, Al ; TURKYILMAZ, I: On Laminar Separation at a Corner Point in Transonic Flow. In: Journal of Fluid Mechanics 
[Schardin 1934]

SCHARDIN, H: Das Toeplersche Schlierenverfahren / VDI (Verein Deutscher Ingenieure). 1934 (367). - Forschungsheft

[Schardin 1942]

SCHARDIN, H: Die Schlierenverfahren und ihre Anwendungen. Springer-Verlag, 1942. Ergebnisse der exakten Naturwissenschaften Band 20

[Schnieder 1999]

SCHNIEDER, M: Wechselwirkung einer starken und einer schwachen Stoßwelle in reagierender Hochenthalpieströmung / Deutsches Zentrum für Luft- und Raumfahrt, Institut für Aerodynamik und Strömungstechnik. Göttingen, Germany, 1999 (DLR FB 98-31). Forschungsbericht

[Schultz u. Jones 1973]

SCHULTZ, DL ; JONES, TV: Heat-transfer Measurements in Short-duration Hypersonic Facilities / Nort Atlantic Treaty Origanuzation, Advisory Group for Aerospace Research and Development. Department of Engineering Science, University of Oxford, Gt. Britain, 1973 (165). - AGARDograph

[Scortecci u.a. 1998]

SCORTECCI, F ; PAGANUCCI, F ; D'AGOSTINO, L: Experimental Investigatin of ShockWave/Boundary-Layer Interactions over an Artificially Heated Model in Hypersonic FlowMoveable Cowl Control for Increased Hypersonic Performence. In: AIAA 8th International Space Planes and Hypersonics and Technologies Conference, 1998. - Norfolk, VA, USA

[Settles 2001]

SetTles, GS ; Adrian, RJ (Hrsg.) ; GHARIB, M (Hrsg.) ; MerZKIRCH, W (Hrsg.) ; RockWell, D (Hrsg.) ; WHITELAW, JH (Hrsg.): Schlieren and Shadowgraph Techniques, Visualizing Phenomena in Transparent Media. Springer, 2001. - ISBN 3540661557

[Settles u. Bogdonoff 1982]

SETTLES, GS ; BOGDONOFF, SM: Scaling of two- and three-dimensional shock/turbulent boundary-layer interactions at compression corners. In: AIAA Journal 6 (1982), Nr. 20, S. 782-789

[Settles u. a. 1979]

Settles, GS ; Bogdonoff, SM ; FitZPATRICK, TJ: Detailed study of attached and separated compression corner flowfields in high Reynolds number supersonic flow. In: AIAA Journal 6 (1979), Nr. 17, S. 579-585

[Simeonides u. Haase 1995]

SIMEONIDES, G; HAASE, W: Experimental and computational investigations of hypersonic flow about compression ramps. In: Journal of Fluid Mechanics (1995), Nr. 283, S. 17-42

[Sinha u. a. 1999]

SINHA, K; WRIGHT, MJ ; CANDLER, GV: The effect of turbulence on double-cone shock 
interactions. In: 37th AIAA Aerospace Sciences Meeting and Exhibit, 1999. - Reno, NV, USA

\section{[Smith 1997]}

SMITH, BA: Weather May Hamper X-38 Drop Tests. In: Aviation Week \& Space Techology (1997), Nr. 147(24):33

[Sonntag 2005]

SONNTAG, J: Kalibirerung einer statischen Sonde für den Hochenthlapiekanal Göttingen / Kulite. 2005. - PDF-Document. erhältlich bei www.kulite.com

[Stalker 1966]

STALKER, RJ: The free-piston shock tube. In: The Aeronautic Quarterly 17 (1966), S. $351-370$

[Stalker 1967]

STALKER, RJ: A study of the free-piston shock tunnel. In: AlAA Journal 5 (1967), Nr. 12, S. 2160-2165

[Stalker 1972]

STALKER, RJ: Development of a hypervelocity wind tunnel. In: Journal of the Royal Aeronautical Society 76 (1972), S. 374-284

[Stalker 1989]

STALKER, RJ: Approximations for Nonequilibrium Hypervelocity Aerodynamics. In: AIAA journal 27 (1989), December, Nr. 12, S. 1761-1769

[Stalker u. Morgan 1988]

StALKeR, RJ ; MORGAN, RG: The University of Queensland Free Piston Shock Tunnel T4 Initial Operation and Preliminary Calibration. (1988)

[Stalker u. Plumb 1968]

STALKER, RJ ; PLUMB, DL: Diaphragm-type shock tube for high shock speeds. In: Nature 218 (1968), S. 789-790

[Stewartson u. Williams 1969]

SteWARtSon, K ; Williams, PG: Self-induced seperation. In: Proceedings of the Royal Society of London (1969), Nr. 312, S. 181-206

[Stewartson u. Williams 1973]

SteWARTSON, K ; WILliams, PG: On self-induced seperation II. In: Mathematika (1973), Nr. 20, S. 98-108

[Sutton u. Graves 1972]

SUTTON, K ; GRAVES, AR: A general stagnation-point heating equation for arbitrary gas mixtures / NASA. 1972 (R-376). - TR 
[Tauber 1989]

TAUBER, ME: A Review of High-Speed, Convective, Heat-Transfer Computation Methods / National Aeronautics and Space Administration (NASA), Office of Managment, Scientific and Technical Information Division. 1989 (2914). - Technical Paper

[Thurow u. a. 2002]

THUROW, B; HILEMAN, J ; LEMPERT, W ; SAMIMY, M: A technique for real-time visualization of flow structure in high-speed flows. In: Physics of Fluids 14 (2002), Nr. 10, S. 34493452

[Tijdeman 1977]

TIJDEMAN, H: Investigations of the Transonic Flow Around Oscillating Airfoils. The Netherlands, Technische Hogeschool Delft, Diss., 1977. - auch NLR TR 77090

[Toepler 1864]

TOEPLER, A: Beobachtungen nach einer neuen optischen Methode - Ein Beitrag zur Experimentalphysik. Max Cohen u. Sohn, 1864. - Bonn

[Tribot u. a. 1999]

Tribot, JP ; Tran, P ; PAllegolX, JF ; Orlowski, M ; BrüCK, S; ANdRes, OP ; FitzGeRALD, SM: X-38 Aerothermodynamics. In: Atmospheric Reentry Vehicle and Systems Symposium, 1999. - Arcachon, France

[Trolinger 1979]

TROLINGER, JD: Application of generalized phase control during reconstruction to flow visualization holography. In: Applied Optics (1979), Nr. 18 (6), S. 766-774

[VDI 1998]

VDI: Wärmeatlas. VDI (Verein Deutscher Ingenieure), 1998. - 5.te Auflage

[Verant 1995]

VERANT, JL: Numerical Enthalpies Rebuilding for Perfect Gas and Nonequilibrium Flows. Applications to High Enthalpy Wind-Tunnels / Office National d'Études et de Recherches Aérospatiales. 1995 (ONERA Report RT No. 69/6121 SY, HT-TN-E-1-201-ONER). - Forschungsbericht

[Vest 1979]

VEST, CM ; (Hrsg.): Holographic Interferometry. John Wiley and Sons Inc, 1979 (- ISBN 0471906832)

[Vincenti u. Kruger 1975]

VINCENTI, WG ; KRUGER, CH: Introduction to Physical Gas Dynamics. Krieger Publishing Comp., Melbourne, Fl. : John Wiley, New York and London/Sydney, 1965, 1975

[Wang u. a. 2001]

WANG, WL ; BOYd, ID ; CANDler, GV ; NOMPelis, I: Particle and Continuum Computations of Hypersonic Flow over Sharp and Blunted Cones. In: 35th AIAA Thermophysics Conference, 2001. - Anaheim, CA, USA 
[Weiland u. a. 1999]

Weiland, MKH ; Hannemann, K ; SChnieder, $M$; BeCK, WH: Heat Flux and Pressure Measurements on HALIS Model in HEG-DLR High Enthalpy Shock Tunnel / Deutsches Zentrum für Luft- ud Raumfahrt e.V. 1999 (DLR-IB 223-99 A 06). - Forschungsbericht

[Weilmuenster u. a. 1994]

Weilmuenster, KJ ; GNOFfo, PA ; Geene, FA: Navier-Stokes simulations of orbiter aerodynamic characteristics including pitch trim and bodyflap. In: Journal of Spacecraft and Rockets 3 (1994), Nr. 31, S. 355-366

[Weilmuenster u. a. 1993]

Weilmuenster, KJ ; GNOfFo, PA ; Greene, FA: Navier-Stokes Simulations of the Shuttle Orbiter Aerodynamic Characteristics with Emphasis on Pitch Trim and Body Flap. In: 28th Thermophysics Conference, 1993. - Orlando, FL, USA

[Woods u. a. 1983]

WOODS, WC ; ARRINGTON, JP ; HAMILTON, HH: A review of preflight estimates of real-gas effects on space shuttle aerodynamic characteristics / NASA. 1983 (CP-2283). - Shuttle Performace: Lessons Learned, Part 1. Langley, VA, USA

[Zaidel' u. a. 1969]

Zaidel', AN ; OstrovskAYA, GV ; OstrovskII, Yl: Plasma diagnostics by holography (a review). In: Sov. Phys. Tech. Phys. (1969), Nr. 13, S. 1153-1164 


\section{A Thermoelementkalibrierung}

Um eine Einschätzung über die Fehler bei der Bestimmung der Wärmestromdichte aus den mit den Thermoelementen gemessenen Temperaturverläufen zu erhalten, ist es erforderlich, die Stoffwerte des Thermoelementmaterials zu kennen (s.a. Anhang B.1). Über die Genauigkeit der Stoffwerte macht der Hersteller der Thermoelemente keine Angaben, so dass mit Annahmen gearbeitet wurde (Kastell (1997), Schnieder (1999)). Für die Ermittlung dieser Stoffwerte wurde eine Kalibriervorrichtung aufgebaut.

Bringt man zwei Körper instantan in Berührung, folgt mit der Annahme einer nur eindimensionalen Wärmeleitung, dass die sich einstellende Kontakttemperatur zeitunabhängig ist (Jessen (1993)). Diese Kontakttemperatur ist die Temperatur, die beide Körper nach dem Erreichen eines thermischen Gleichgewichtszustandes nach unendlich langer Zeit annehmen würden. Die Kontakttemperatur $T_{K}$ hängt von den Anfangstemperaturen beider Körper und der Dichte $\rho$, spezifischer Wärmekapazität $c$ und der Wärmeleitfähigkeit $k$ beider Körper ab. Dieser Zusammenhang ist durch

$$
\frac{T_{K}-T_{1}}{T_{2}-T_{K}}=\frac{\sqrt{(\rho \cdot c \cdot k)_{2}}}{\sqrt{(\rho \cdot c \cdot k)_{1}}}
$$

gegeben. Mit der Kenntnis der Stoffwerte des einen Körpers kann man durch die Messung der Temperaturen $T_{K}, T_{1}$ und $T_{2}$ das Produkt der Stoffwerte $\sqrt{\rho \cdot c \cdot k}$ des anderen Körpers bestimmen. Somit kann der für die Wärmestromdichtebestimmung benötigte Wert (s. Abschnitt 4.2.3, Gl. 4.12) ermittelt werden. Die experimentelle Umsetzung erfolgt mit Wasser als bekanntem Kontaktpartner. Mit einer Flüssigkeit kann eine gute Kontaktierung gewährleistet werden, und gerade die Stoffwerte von Flüssigkeiten sind in genügender Genauigkeit bekannt (VDI (1998)).
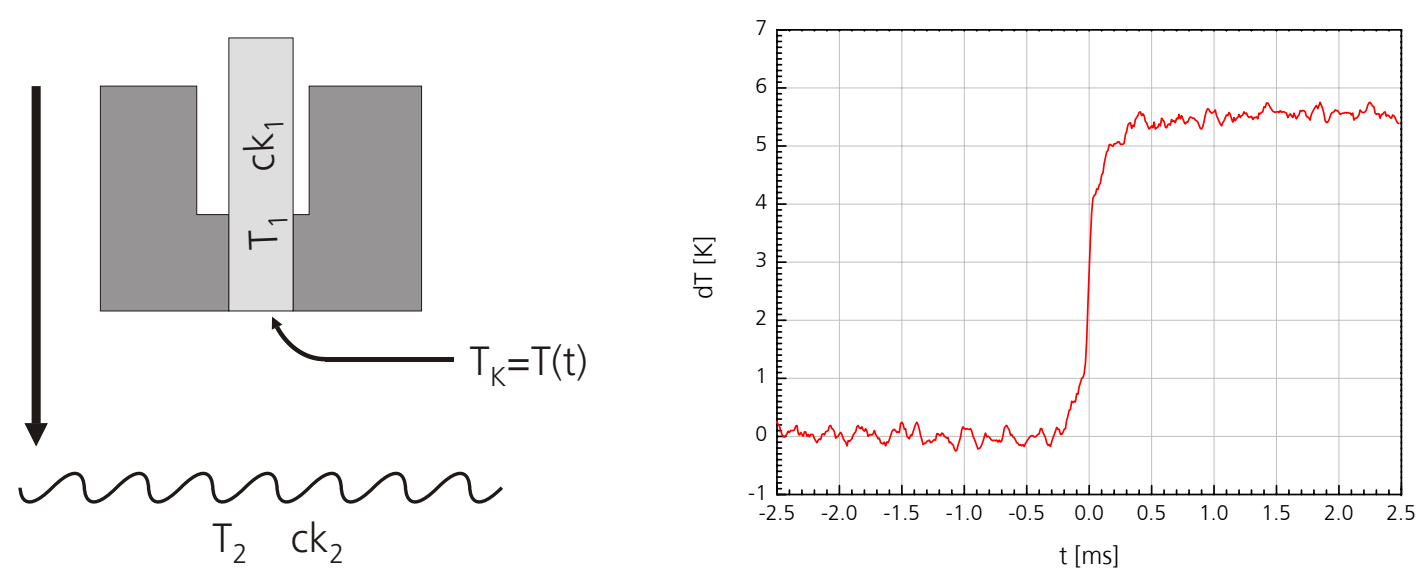

Abbildung A.1: Schema für den Versuchsaufbau bei der Kontaktkalibrierung der Thermoelemente. Der Eintauchvorgang ist auf der linken Seite, ein Messsignal auf der rechten Seite dargestellt. 


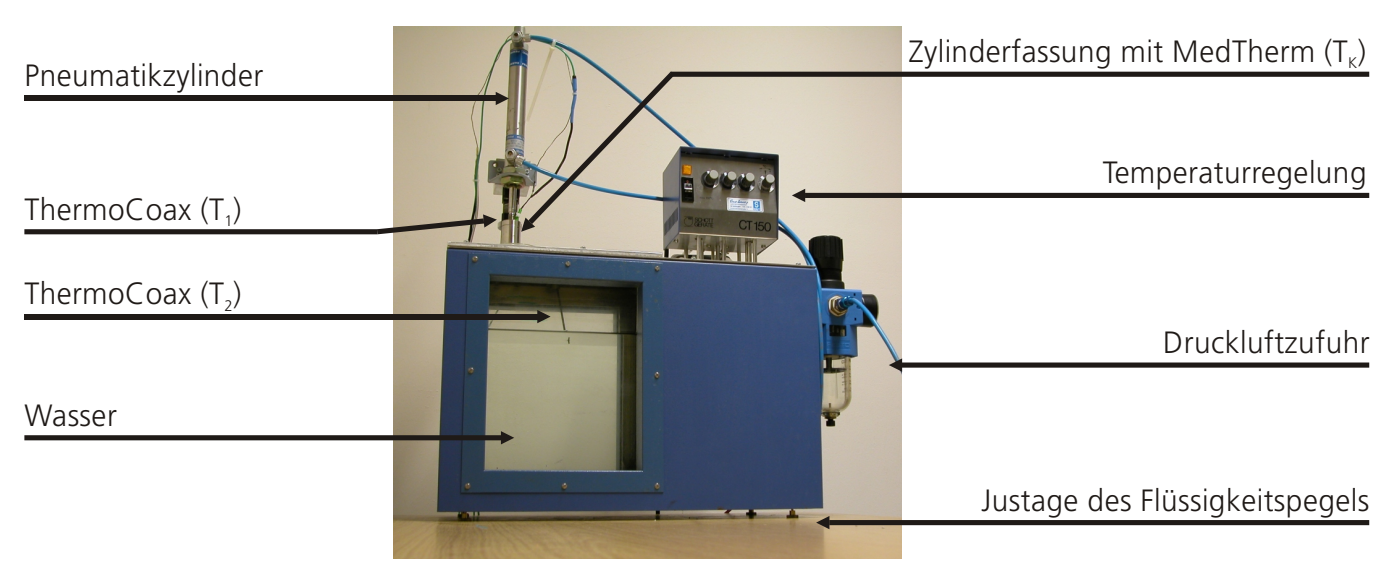

Abbildung A.2: Photographische Ansicht des Aufbaus für die Kontaktkalibrierung.

Im experimentellen Aufbau wird das Thermoelement in eine zylindrische Fassung montiert, wie in Abb. A.1 auf der linken Seite im Schema dargestellt. Für die Kalibrierung der Thermolelemente muss die Messung der Kontakttemperatur beim Eintauchvorgang in das Wasser durch das zu kalibrierende Thermoelement schnell erfolgen, die Messung von $T_{1}$ und $T_{2}$ kann mit langsameren Aufnehmern ${ }^{1}$ stattfinden. Die Annahme der eindimensionalen Wärmeleitung wird durch die Kontakttemperaturmessung selbst geprüft: Wird kein Sprung auf eine konstante Temperatur gemessen, so ist die Voraussetzung der Messmethode nicht erfüllt. In Abb. A.1 ist als Beispiel eine Messung aufgetragen.

Die Zylinderfassung mit dem Thermoelement wird durch einen pneumatischen Zylinder mit hoher Geschwindigkeit auf die Wasseroberfläche geschossen. Der Zylinder hat eine Höhenjustierung, die es erlaubt, die Zylinderoberfläche so mit der Wasseroberfläche in Kontakt zu bringen, dass die Wasserbewegung unter dem Zylinder minimiert wird. So kann der konvektive Anteil der Wärmeübertragung, der von der Flüssigkeitsbewegung stammt, bei der Messung des Sprungs minimiert werden. Hierzu ist auch eine Justage des Wasserpegels an dem Wasserbad vorhanden, welches den gesamten Versuchsaufbau trägt. In Abb. A.2 ist der Aufbau komplett dargestellt.

Um das Verhalten bei verschiedenen Wassertemperaturen zu untersuchen, ist das Wasserbad heizbar. Die Messreihen, die bis $80^{\circ} \mathrm{C}$ aufgenommen wurden, zeigten keinen Einfluss auf die Bestimmung der Stoffwerte der Thermoelemente. Allerdings werden mit höherer Wasserbadtemperatur die Fehler bei der Bestimmung der Temperaturen kleiner, und damit wird die Reproduzierbarkeit der Messungen verbessert. Für die hier vorgestellten Ergebnisse der Kalibrierung wurde eine Wasserbadtemperatur von $70^{\circ} \mathrm{C}$ benutzt, da bei höheren Temperaturen das Wasser nahe der Siedegrenze Flüssigkeitsbewegungen und zu große Gradienten in der Temperaturverteilung aufwies.

${ }^{1}$ TKI 20/10/NN (Typ K), Seriennummern:3074,4668,4343, ThermoCoax SAS, Flers Cedex, Frankreich [www.thermocoax.com] 


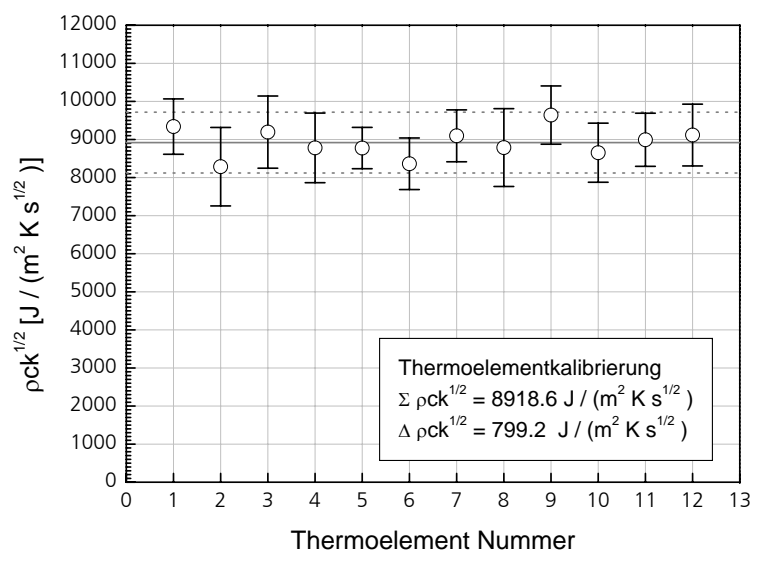

\begin{tabular}{|ccc|}
\hline No & $\rho^{\mathbf{c k}} \mathbf{1}^{\mathbf{2}}$ & $\sigma\left(\rho \mathbf{c k}{ }^{\mathbf{1 / 2}}\right)$ \\
\hline 1 & 9338.68 & 726.24 \\
2 & 8286.15 & 1030.11 \\
3 & 9194.11 & 948.21 \\
4 & 8779.31 & 913.43 \\
5 & 8775.79 & 542.47 \\
6 & 8362.23 & 676.91 \\
7 & 9096.05 & 681.07 \\
8 & 8787.36 & 1021.54 \\
9 & 9641.35 & 765.01 \\
10 & 8652.75 & 776.23 \\
11 & 8992.81 & 697.21 \\
12 & 9116.57 & 811.63 \\
\hline$\Sigma$ & 8918.60 & 799.17 \\
\hline
\end{tabular}

Abbildung A.3: Ergebnis der Thermoelementkalibrierung.

In Abb. A.3 sind die Ergebnisse der Kalibrierung aufgetragen. Der abgeschätzte Fehler der Einzelmessungen (s. Anhang B.4) ist kleiner als die Standardabweichung für 20 Experimente mit einem Sensor, und deshalb wird diese als Fehler für das Produkt der Stoffwerte angenommen. Der Mittelwert der Standardabweichung für 12 verschiedene Aufnehmer geht somit als Fehler für den $\sqrt{\rho \cdot c \cdot k}$ Wert aller Thermoelemente in die Bestimmung der Wärmestromdichte ein. Die graphische Darstellung der Kalibrierungsergebnisse in Abb. A.3 zeigt deutlich, dass eine individuelle Kalibrierung der Thermoelemente nicht möglich ist. 


\section{B Fehlerrechnungen}

\section{B.1 Wärmestromdichtemessung mittels koaxialer Thermoelemente}

Für die in Abschnitt 4.2.3 angegebene Differentialgleichung

$$
\frac{\partial^{2} T}{\partial x^{2}}=\frac{1}{a} \frac{\partial T}{\partial t}
$$

ergibt sich die exakte Lösung unter Annahme der Messung einer konstanten Wärmestromdichte $\dot{q}_{S} \mathrm{zu}$

$$
T_{W}(x=0, t)=\frac{2 \dot{q}_{S}}{\sqrt{\pi}} \sqrt{\frac{t}{\rho \cdot c \cdot k}} .
$$

Die umgestellte Form dieser Lösung

$$
\dot{q}_{S}=\frac{T_{W}(x=0, t) \cdot \sqrt{\pi}}{2 \cdot \sqrt{t /(\rho \cdot c \cdot k)}}=\frac{T_{W} \cdot \sqrt{\pi} \cdot a}{2 \cdot \sqrt{t}}
$$

kann nach den fehlerbehafteten Größen $T_{W}, t$ und $a=\sqrt{\rho \cdot c \cdot k}$ fortgepflanzt werden:

$$
\begin{aligned}
\frac{\partial \dot{q}_{S}}{\partial T_{W}} & =\frac{\sqrt{\pi} \cdot a}{2 \cdot \sqrt{t}} \\
\frac{\partial \dot{q}_{S}}{\partial t} & =-\frac{T_{W} \cdot \sqrt{\pi} \cdot a}{4 \cdot \sqrt{t^{3}}} \\
\frac{\partial \dot{q}_{S}}{\partial a} & =\frac{T_{W} \cdot \sqrt{\pi}}{2 \cdot \sqrt{t}}
\end{aligned}
$$

Damit ergibt sich dann für den Gesamtfehler:

$$
\begin{aligned}
\Delta q_{S} & =\left|\frac{\partial \dot{q}_{S}}{\partial T_{W}}\right| \cdot \Delta T_{W}+\left|\frac{\partial \dot{q}_{S}}{\partial t}\right| \cdot \Delta t+\left|\frac{\partial \dot{q}_{S}}{\partial a}\right| \cdot \Delta a \\
& =\frac{\sqrt{\pi} \cdot a}{2 \cdot \sqrt{t}} \cdot \Delta T_{W}+\frac{T_{W} \cdot \sqrt{\pi} \cdot a}{4 \cdot \sqrt{t^{3}}} \cdot \Delta t+\frac{T_{W} \cdot \sqrt{\pi}}{2 \cdot \sqrt{t}} \cdot \Delta a
\end{aligned}
$$

Der Fehler bei der Temperaturmessung mittels Thermoelementen vom Typ $\mathrm{E}$ ist für den verwendeten Temperaturbereich absolut $\Delta T_{W}=0.5 \mathrm{~K}$, und für die Zeitmessung mittels 

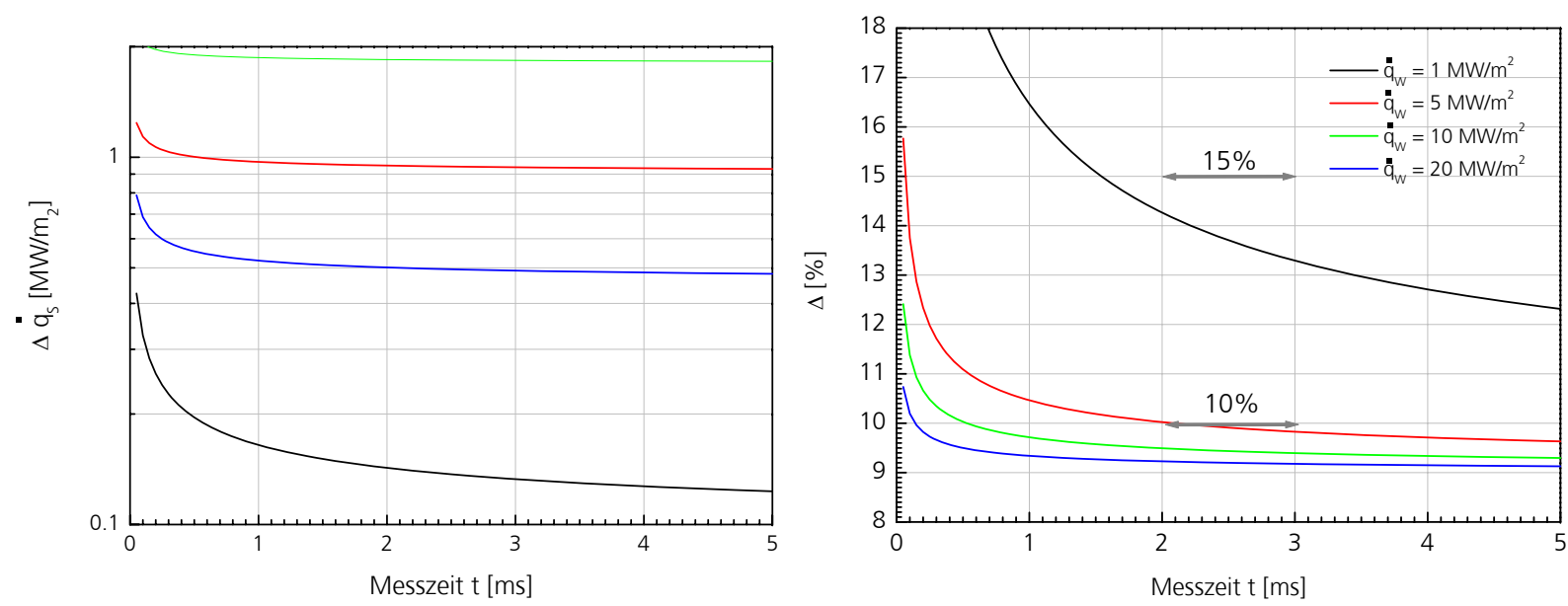

Abbildung B.1: Fehler bei der Messung der Wärmestromdichte für verschiedene als konstant angenommene Wärmestromdichten in das Thermoelement hinein.

des am HEG verwendeten Transientenrekorders kann $\Delta t=10^{-7} \mathrm{~s}$ angenommen werden. Der verwendete Wert für $\rho \cdot c \cdot k$ stammt aus einer Kalibrierung, hier ist $\Delta(\rho \cdot c \cdot k)=$ 799.17J $/\left(m^{2} \cdot K \cdot \sqrt{s}\right)$ ermittelt worden (s.a. Abschnitt A im Anhang).

In der Abb. B.1 sind die absoluten und relativen Fehler für verschiedene als konstant angenommen Wärmestromdichten in das Thermoelement aufgetragen. Auf der linken Seite der Abb. B.1 sind die absoluten Fehler über der Messzeit aufgetragen und auf der rechten Seite die relativen Fehler. Innerhalb der typischen Messzeit im HEG strebt der Fehler bei der Bestimmung der Wärmestromdichte gegen 5\% des Messwertes. Dies war zu erwarten, da der Fehler des $\sqrt{\rho \cdot c \cdot k}$-Wertes linear in die Bestimmung der Wärmestromdichte eingeht (s. Abschnitt 4.2.3, Gl. 4.12) und damit den Fehler nach genügend langer Zeitdauer der Messung bestimmt. Je höher die zu messende Wärmestromdichte, desto schneller strebt der Fehler gegen diese Grenze, da die durch den Wärmstrom entstehende Temperatur des Sensors größer wird und damit der absolute Fehler der Temperaturmessung von $\Delta T_{W}=0.1$ $\mathrm{K}$ keinen großen Einfluss mehr hat.

\section{B.2 Bestimmung der Ablöselänge}

Die Bestimmung der Ablöselänge für die Versuche mit der Doppelrampe erfolgt mittels der Größen $L_{u}, L_{R}, \Theta_{1}$ und $\Theta_{2}$. Die Länge $L_{u}$ (Ablöseort bis Scharnier) und die Länge $L_{R}$ (Scharnier bis Wiederanlegeort) werden mit einem Bildverarbeitungsprogramm aus den aufgenommenen Schlierenbildern bestimmt. Der Fehler wird hier auf $\Delta L_{u}=1 \mathrm{~mm}$ und $\Delta L_{R}=$ $1 \mathrm{~mm}$ geschätzt. Die Anstellwinkel der beiden Rampen werden vor dem Versuch mit einer Digitalwaage ${ }^{1}$ bestimmt. Der Fehler ist hier maximal $\Delta \Theta_{1}=0.15^{\circ}$ und $\Theta_{2}=0.15^{\circ}$. Für die Ablöselänge gilt

$$
L_{\text {sep }}=\sqrt{\left(L_{u} \cos \Theta_{1}+L_{R} \cos \Theta_{2}\right)^{2}+\left(L_{u} \sin \Theta_{1}+L_{R} \sin \Theta_{2}\right)^{2}},
$$

\footnotetext{
${ }^{1}$ Model Clinotronic, Serien-Nummer: 003123, Wyler AG, Winterthur, Schweiz
} 
für die Fehlerfortpflanzung

$$
\Delta L_{\text {sep }}=\left|\frac{\partial L_{\text {sep }}}{\partial L_{u}}\right| \cdot \Delta \partial L_{u}+\left|\frac{\partial L_{\text {sep }}}{\partial L_{R}}\right| \cdot \Delta L_{R}+\left|\frac{\partial L_{\text {sep }}}{\partial \Theta_{1}}\right| \cdot \Delta \Theta_{1}+\left|\frac{\partial L_{s e p}}{\partial \Theta_{2}}\right| \cdot \Delta \Theta_{2},
$$

und für die Ableitungen nach den Messgrößen

$$
\begin{aligned}
\frac{\partial L_{\text {sep }}}{\partial L_{u}} & =\frac{L_{u}+\left(\cos \Theta_{1} \cdot \cos \Theta_{2}+\sin \Theta_{1} \cdot \sin \Theta_{2}\right) \cdot L_{R}}{\sqrt{L_{u}^{2}+2 L_{u}\left(\cos \Theta_{1} \cdot \cos \Theta_{2}+\sin \Theta_{1} \cdot \sin \Theta_{2}\right) \cdot L_{R}+L_{R}^{2}}} \\
\frac{\partial L_{\text {sep }}}{\partial L_{R}} & =\frac{L_{R}+\left(\cos \Theta_{1} \cdot \cos \Theta_{2}+\sin \Theta_{1} \cdot \sin \Theta_{2}\right) \cdot L_{u}}{\sqrt{L_{R}^{2}+2 L_{R}\left(\cos \Theta_{1} \cdot \cos \Theta_{2}+\sin \Theta_{1} \sin \cdot \Theta_{2}\right) \cdot L_{u}+L_{u}^{2}}} \\
\frac{\partial L_{s e p}}{\partial \Theta_{1}} & =\frac{\left(\cos \Theta_{1} \cdot \sin \Theta_{2}-\sin \Theta_{1} \cdot \cos \Theta_{2}\right) \cdot L_{u} \cdot L_{R}}{\sqrt{2 \cdot \cos \Theta_{1} \cdot \cos \Theta_{2} \cdot L_{u} \cdot L_{R}+2 \cdot \sin \Theta_{1} \cdot \sin \Theta_{2} \cdot L_{u} \cdot L_{R}+L_{u}^{2}+L_{R}^{2}}} \\
\frac{\partial L_{s e p}}{\partial \Theta_{2}} & =\frac{\left(\cos \Theta_{2} \cdot \sin \Theta_{1}-\sin \Theta_{2} \cdot \cos \Theta_{1}\right) \cdot L_{u} \cdot L_{R}}{\sqrt{2 \cdot \cos \Theta_{2} \cdot \cos \Theta_{1} \cdot L_{u} \cdot L_{R}+2 \cdot \sin \Theta_{2} \cdot \sin \Theta_{1} \cdot L_{u} \cdot L_{R}+L_{u}^{2}+L_{R}^{2}}} .
\end{aligned}
$$

\section{B.3 Bestimmung der Ruheenthalpie $h_{0}$}

Die Bestimmung der Ruheenthalpie im Reservoir des HEG erfolgt unter der Verwendung der Korrelation von Verant (1995) in Abhängigkeit von den Messgrößen des Pitot-Druckes in der Anströmung $p_{t 2}$ und der Staupunktwärmestromdichte $q_{t 2}$ auf einer Halbkugel mit dem Radius $R$ :

$$
h_{0}=R_{g} \cdot T_{r e f}\left(\frac{q_{t 2}}{23.787} \cdot \sqrt{\frac{R}{p_{t 2}}}\right)^{\frac{1}{1.0688}}+h_{\text {wall }} .
$$

Die Ableitungen nach den fehlerbehafteten Größen sind

$$
\begin{aligned}
\frac{\partial h_{0}}{\partial q_{t 2}} & =\frac{0.0048235 \cdot R_{g} \cdot T_{r e f} \cdot \sqrt{\frac{R}{p_{t 2}}}}{\left(q_{t 2} \cdot \sqrt{\frac{R}{p_{t 2}}}\right)^{0.064371}}, \\
\frac{\partial h_{0}}{\partial R} & =\frac{0.024117 \cdot q_{t 2} \cdot R_{g} \cdot T_{r e f}}{p_{t 2} \cdot \sqrt{\frac{R}{p_{t 2}}} \cdot\left(q_{t 2} \cdot \sqrt{\frac{R}{p_{t 2}}}\right)^{0.006437}}, \\
\frac{\partial h_{0}}{\partial p_{t 2}} & =-\frac{0.024117 \cdot q_{t 2} \cdot R_{g} \cdot T_{r e f} \cdot R}{\left(\sqrt{\frac{R}{p_{t 2}}} \cdot q_{t 2}\right)^{0.064371} \cdot \sqrt{\frac{R}{p_{t 2}}} \cdot p_{t 2}^{2}} .
\end{aligned}
$$




\section{B.4 Kontaktkalibrierungsverfahren}

Für die Bestimmung des Produktes der Stoffwerte bei der Kontaktkalibrierung der Thermoelemente wird die folgende Bestimmungsgleichung benutzt:

$$
\sqrt{(\rho \cdot c \cdot k)_{1}}=\sqrt{(\rho \cdot c \cdot k)_{2}} \cdot \frac{T_{2}-T_{K}}{T_{K}-T_{1}}
$$

Die Ableitungen nach den fehlberbehafteten Größen sind wie folgt gegeben

$$
\begin{aligned}
& \frac{\partial \sqrt{(\rho \cdot c \cdot k)_{1}}}{\partial \sqrt{(\rho \cdot c \cdot k)_{2}}}=\frac{T_{2}-T_{K}}{T_{K}-T_{1}} \\
& \frac{\partial \sqrt{(\rho \cdot c \cdot k)_{1}}}{\partial T_{1}}=-\sqrt{(\rho \cdot c \cdot k)_{2}} \cdot \frac{\left(T_{K}-T_{2}\right)}{\left(T_{1}-T_{K}\right)^{2}} \\
& \frac{\partial \sqrt{(\rho \cdot c \cdot k)_{1}}}{\partial T_{2}}=\frac{\sqrt{(\rho \cdot c \cdot k)_{2}}}{\left(T_{K}-T_{1}\right)} \\
& \frac{\partial \sqrt{(\rho \cdot c \cdot k)_{1}}}{\partial T_{K}}=-\sqrt{(\rho \cdot c \cdot k)_{2}} \cdot \frac{\left(T_{2}-T_{1}\right)}{\left(T_{K}-T_{1}\right)^{2}},
\end{aligned}
$$

und der Gesamtfehler ergibt sich zu

$$
\begin{aligned}
\Delta \sqrt{(\rho \cdot c \cdot k)_{1}} & =\frac{\partial \sqrt{(\rho \cdot c \cdot k)_{1}}}{\partial \sqrt{(\rho \cdot c \cdot k)_{2}}} \cdot \Delta \sqrt{(\rho \cdot c \cdot k)_{2}} \\
& +\sqrt{(\rho \cdot c \cdot k)_{2}} \cdot \frac{\left(T_{K}-T_{2}\right)}{\left(T_{1}-T_{K}\right)^{2}} \cdot \Delta T_{1} \\
& +\frac{\sqrt{(\rho \cdot c \cdot k)_{2}}}{\left(T_{K}-T_{1}\right)} \cdot \Delta T_{2} \\
& +\sqrt{(\rho \cdot c \cdot k)_{2}} \cdot \frac{\left(T_{2}-T_{1}\right)}{\left(T_{K}-T_{1}\right)^{2}} \cdot \Delta T_{K} .
\end{aligned}
$$




\section{Lebenslauf}

Name:

Anschrift:

Telefon:

E-Mail:

Geb.-Datum:

Geb.-Ort:

Familienstand:

Staatsbürgerschaft:

\section{Schulbildung}

1979-1989

\section{Zivildienst}

1989-1990

\section{Studium}

1990-1997
Jan Martinez Schramm

Über den Höfen 30, 37077 Göttingen

055176972

Jan.Martinez@dlr.de

25. Oktober 1968

Göttingen

verheiratet, 3 Kinder (geboren 1997, 2000 sowie 2003)

deutsch

Abitur am Theodor-Heuss-Gymnasium in Göttingen

Universitätsklinikum in Göttingen

Physik an der Georg-August-Universität Göttingen

Diplomnote: "Gut"

Thema der Diplomarbeit:

„Experimentelle Untersuchung der Überschallströmung im

Freistrahl einer gepulst betriebenen Düse"

\section{Berufliche Tätigkeit am Deutschen Zentrum für Luft- und Raumfahrt e.V.}

1990-1997

1997-1999

1999-2002

ab 2002
Studentische Hilfskraft

Doktorandenstipendium

Mitarbeiter im wiss. Dienst (Jungwissenschaftler)

Mitarbeiter im wiss. Dienst 